\title{
NANOIMPRINT LITHOGRAPHY
}

\section{FOR}

\section{SOLAR CELL TEXTURISATION}

\section{Dissertation}

zur Erlangung des akademischen Grades des

Doktors der Ingenieurwissenschaften (Dr. Ing.)

der

Fakultät für Angewandte Wissenschaften der

Albert-Ludwigs-Universität Freiburg i. Br.

vorgelegt von

Hubert Hauser

\author{
Angefertigt am \\ Fraunhofer-Institut für \\ Solare Energiesysteme \\ 2013
}



Dekan: Prof. Dr. Y. Manoli

Referent: Prof. Dr. H. Reinecke Korreferent: Prof. Dr. G. Willeke

Datum der Promotion: 10.06.2013 



\section{Table of contents}

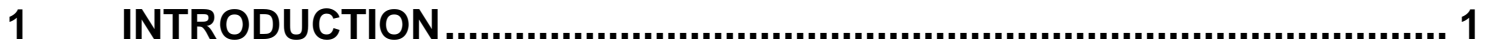

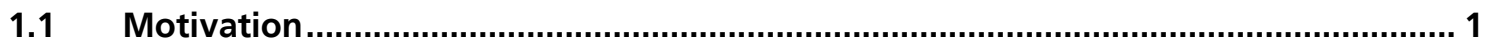

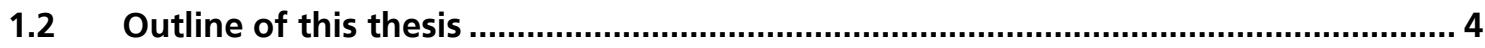

2 WAFER BASED SILICON SOLAR CELLS ........................................ 7

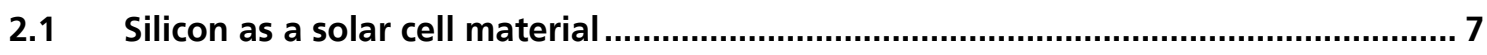

2.1.1 Crystal structure and implications on optical properties....................................... 7

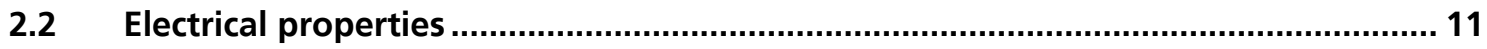

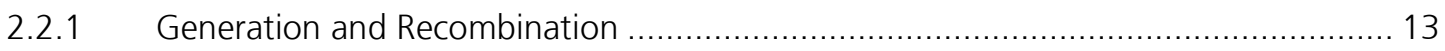

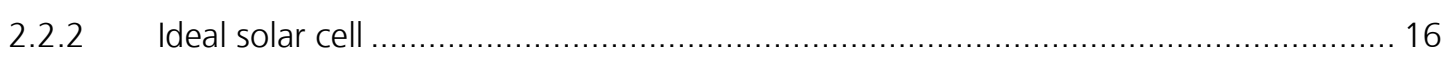

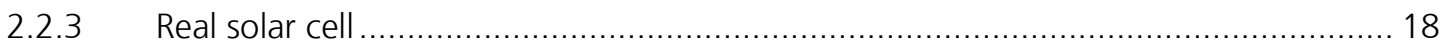

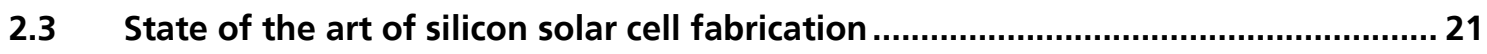

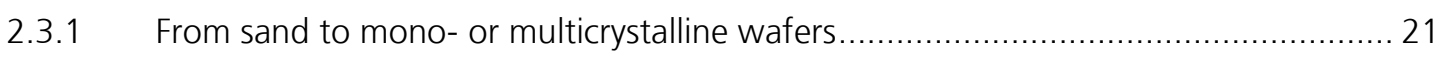

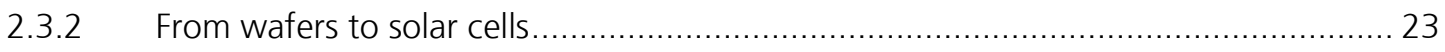

2.3.3 Laboratory scale processes on the verge of an industrial realisation......................... 25

3 TEXTURED SILICON SURFACES - OPTICAL \& ELECTRICAL EFFECTS................................................................................................. 28

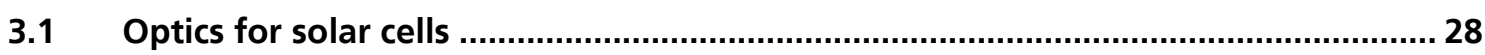

3.1.1 Two effects: Reduction of reflectivity and light trapping ................................... 28

3.1.2 Evolution of surface texturing for silicon solar cells............................................. 37

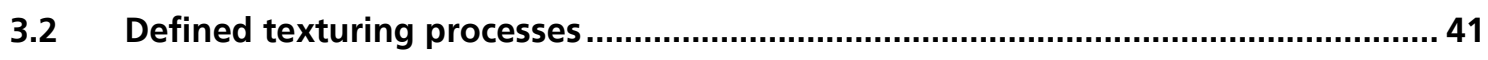

3.2.1 Honeycomb texturing of multicrystalline silicon...................................................... 44

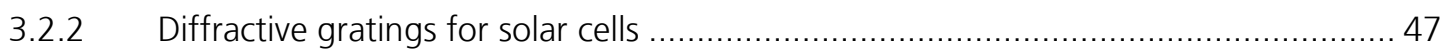

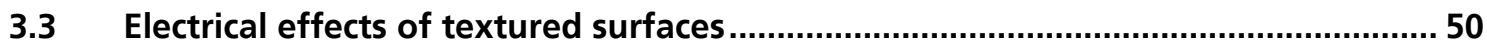

3.3.1 General considerations - a review of studies on alkaline etching of monocrystalline silicon 50

3.3.2 Effects implied by other texturing processes on multicrystalline silicon ..................... 54

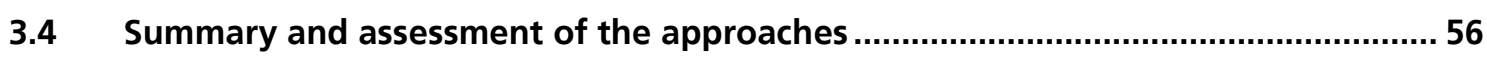

4 TEXTURING SCHEME DEVELOPED WITHIN THIS WORK ............... 58 
4.1 Overview of the developed process chain ............................................................... 58

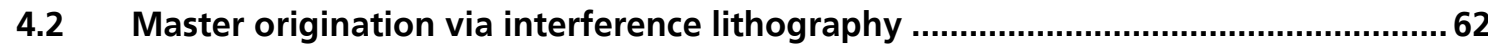

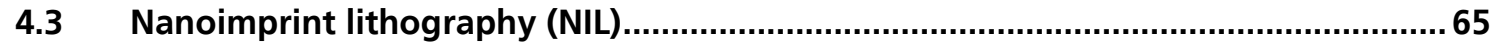

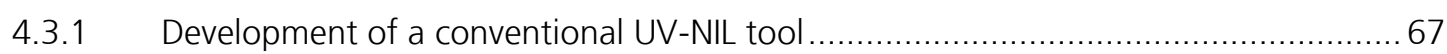

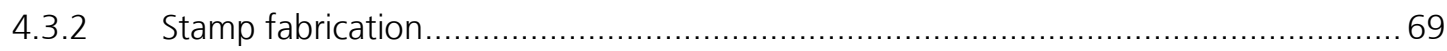

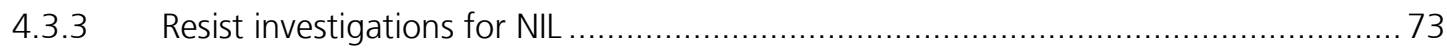

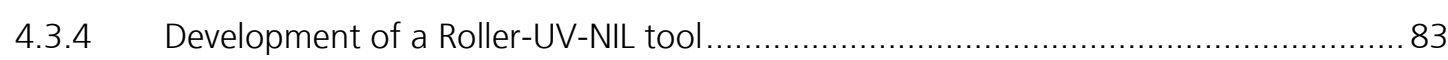

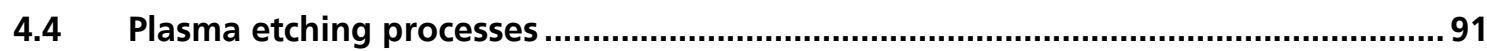

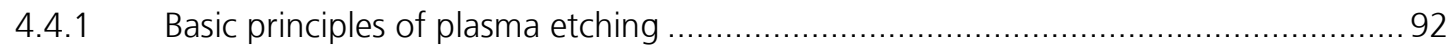

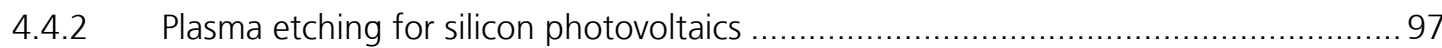

5 TWO CONCEPTS BASED ON DEFINED TEXTURES ......................... 100

5.1 Honeycomb textured multicrystalline silicon solar cells ......................................... 100

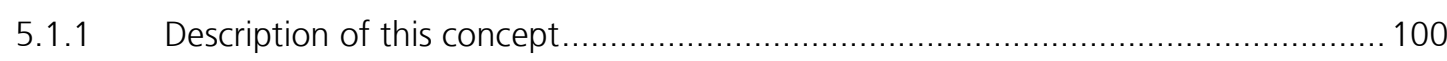

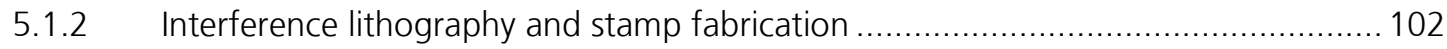

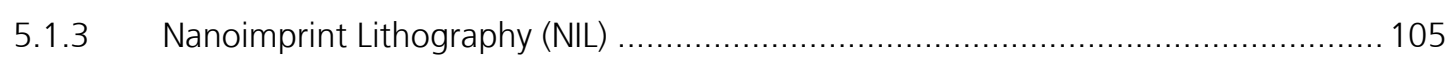

5.1.4 Plasma etching and resist removal ..................................................................... 111

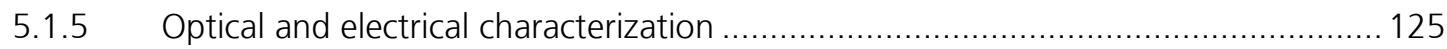

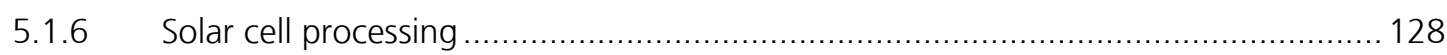

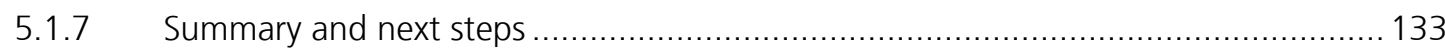

5.2 Diffractive gratings on the back side of silicon solar cells ..................................... 135

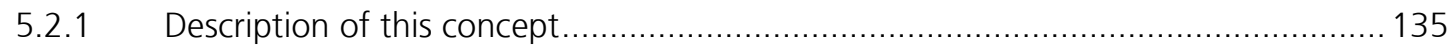

5.2.2 Interference lithography and stamp fabrication ................................................ 137

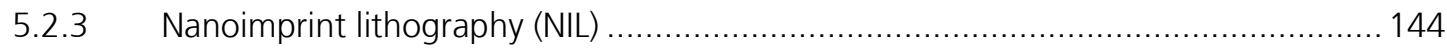

5.2.4 Plasma etching and resist removal .................................................................... 146

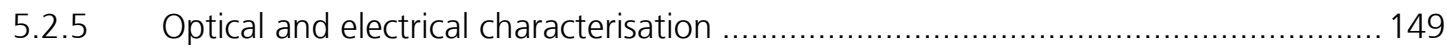

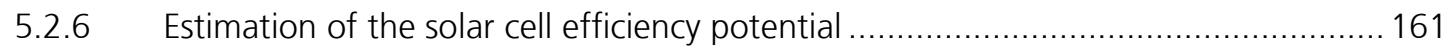

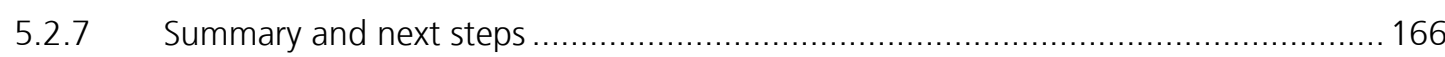

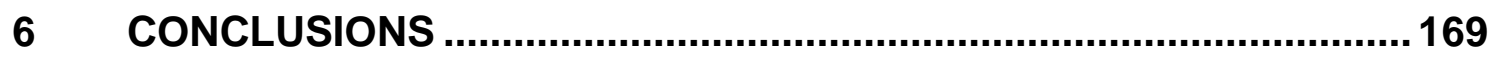

7 DEUTSCHE ZUSAMMENFASSUNG ............................................. 173

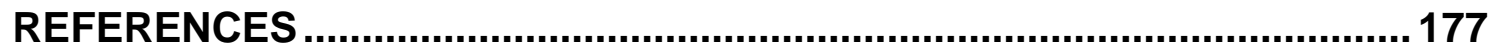

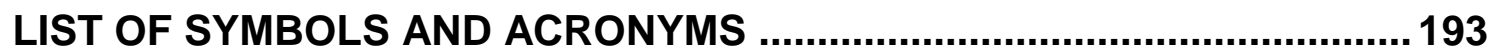


LIST OF PUBLICATIONS AND PATENTS

DANKSAGUNG. 



\section{Introduction}

\subsection{Motivation}

The sun delivers a huge amount of energy to the earth. As this resource of energy comes for free, its conversion into electrical energy via photovoltaics (PV) is extremely tempting. In the last decades the field of PV evolved from a rather academic to a large scale industrial one. There is currently huge pressure on manufacturers to reduce costs and to open up an economic way of providing energy via PV. The current market is dominated by wafer-based silicon solar cells.

To meet this demand of cost-effectiveness two, important trends (amongst others) can be followed:

(i) The use of cheaper substrates

(ii) Reduction of material consumption

The first demand is followed by the use of multicrystalline silicon, which requires less energy in fabrication than the growing of monocrystalline material. This material makes up for the largest share of the photovoltaics market (with $57 \%$ share of the $37 \mathrm{GW}_{\text {peak }}$ fabrication in 2011; see Figure 1.1) [1]. One approach toward the second demand is to reduce the silicon material itself. There has been a steady reduction in the wafer thickness for PV over the last years and the forecast of the International Technology Roadmap for Photovoltaics (ITRPV) predicts a continuation of this trend (Figure 1.2) [2]. Beyond this prediction, which covers conventional wire sawing developments, there are various concepts to realise even far thinner substrates down to well below $50 \mu \mathrm{m}[3,4]$.

However, cost-effectiveness is not only achievable by reducing fabrication costs. Another important parameter is the conversion efficiency of solar cells. This parameter is in conflict with the measures described before. Besides the reduced electrical quality when using cheaper multicrystalline silicon, this conflict is strongly related to optics. 


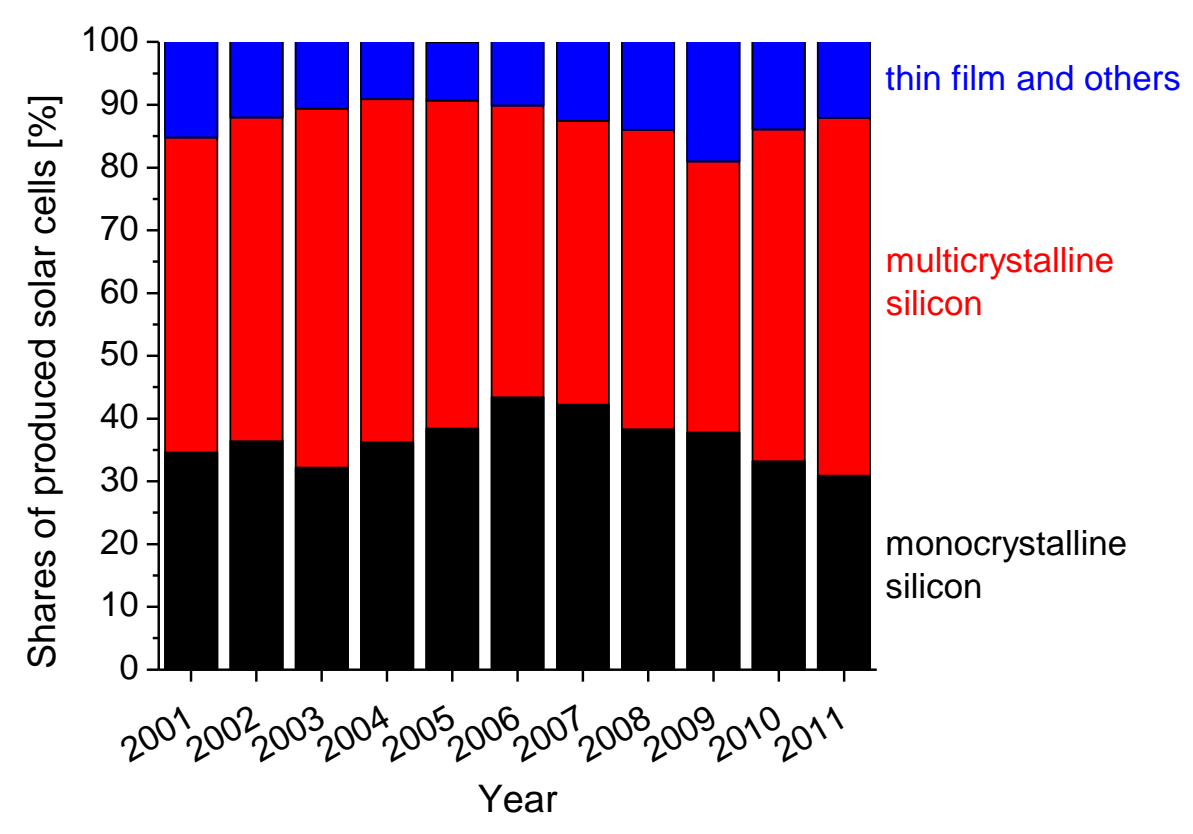

Figure 1.1: Share of solar cell materials in mass production. All technologies other than the two main contributors (multi- and monocrystalline wafer-based solar cells) are combined under "thin film and others". Besides classical thin film PV, this also includes alternative wafering technologies like ribbon sheet approaches, as well as III-V semiconductors and dye- or organic PV. Data is taken from [1].

First, the current state-of-the-art of texturing multicrystalline silicon (the so-called isotexture) is less effective than pyramidal textures, which are only feasible on monocrystalline silicon. To improve the optical behaviour, the honeycomb texture was introduced for reaching the highest efficiencies on this material [5]. However, the need of photolithography for the realisation of this defined, regular pattern prohibited a transfer of this concept into industrial environments, although the gain in efficiency to be expected is considerable.

The use of thinner wafers will lead to more losses as a result of incomplete absorption. One promising approach to minimise these losses by light trapping effects is based on the use of photonic structures (e.g. diffractive gratings). In this approach, wave optical effects are applied to enhance internal path lengths of light within the solar cell and thus one can speak of increasing its optical thickness. Feature sizes for this aspect are too demanding even for classical photolithography and it therefore was believed impossible to employ such structures in industrial solar cells. 


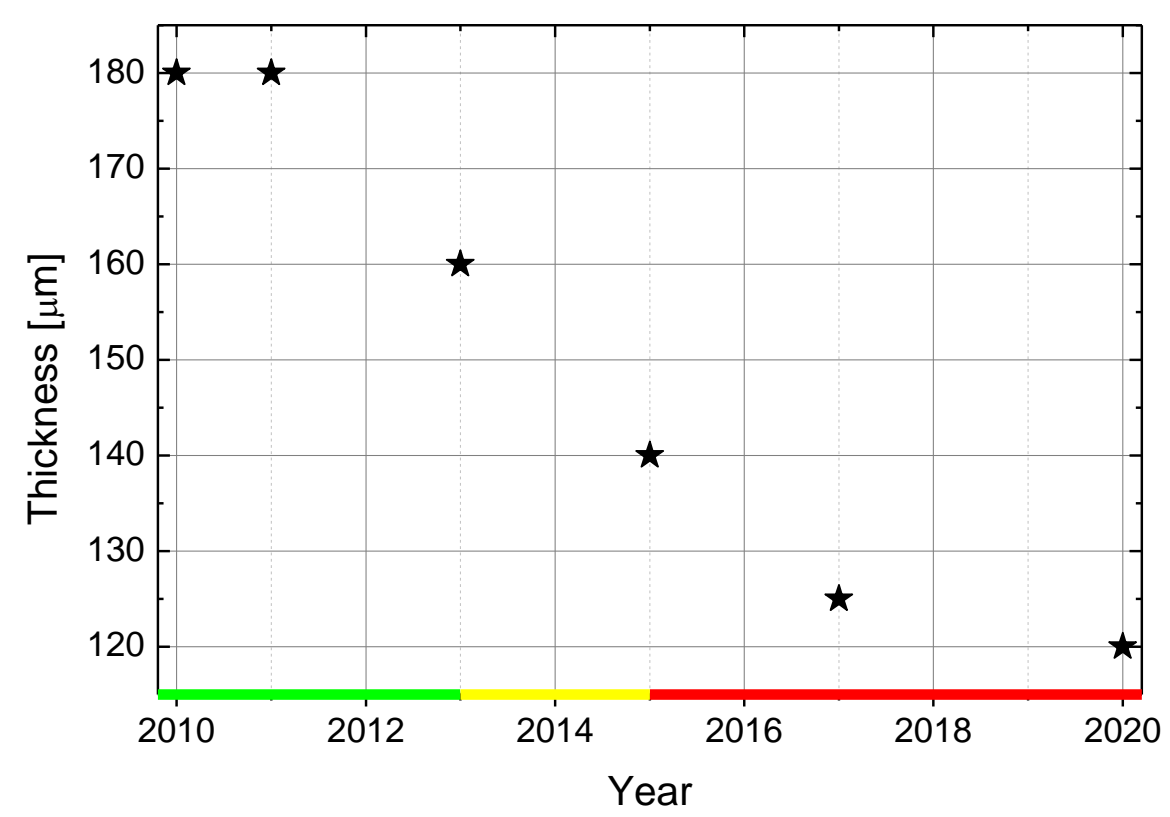

Figure 1.2: Trend of minimum as-cut wafer thickness processed in mass production of solar cells. The colours on the timescale indicate the status of existing solutions for industrial processing (Green: Industrial solution exists and is being optimized in production. Yellow: Industrial solution is known but not yet in mass production. Red: Industrial solution is not known. Data taken from [2].

Within this thesis, nanoimprint lithography (NIL) is investigated as a technology to overcome these limitations. This technology is based on mechanical embossing of a polymeric resist layer, which can then be used as an etching mask to realise defined textures as were described above. The emerging technology of NIL allows the realisation of features down to the sub-100 nm scale since it is not limited by diffraction effects, as the case for photolithography. It was listed as potential technology in the microelectronics industry for future chip generations [6] and as "one of ten emerging technologies that will change the world" by the Technology Review magazine [7].

The demands in microelectronics are different to the ones in the PV industry. Therefore, the scope of this thesis is the development of tools and processes for $\mathrm{NIL}$ adapted to the requirements in PV applications. The main differences besides the cost issue are e.g. large areas to be processed and the use of comparably rough surfaces. To meet these demands, NIL processes were developed on the basis of UV-curing resist materials and elastomeric stamps. The former is motivated by short processing times and the latter by the ability to pattern etching masks on large areas. Besides these general issues about NIL, one key aspect of this work was the development of a Roller-NIL tool to open up the up-scalability of this technology to in-line processing. 
For large area master origination of periodic features, interference lithography was applied. After the structuring of etching masks using NIL, plasma etching processes were applied within this work for the pattern transfer into the silicon substrates, both for the realisation of honeycomb textures as well as diffractive gratings. Plasma etching processes are chosen as they permit the most degrees of freedom in terms of feasible texture geometries. Thereby, the full potential of the NIL technology can be tapped.

\subsection{Outline of this thesis}

In chapter 2, the basic principle of solar cells is described. To this end, the semiconductor silicon as a solar cell material and the state-of-the-art of solar cell processing is introduced. At the end of this chapter, processes that are established in laboratory environments and are on the verge of implementation in industrial fabrication are explained. These processes are also later applied to the fabrication of solar cells within this work. Throughout the description of these general aspects, optical effects related to the solar cell performance are highlighted.

Optical effects are studied in depth in chapter 3, where strategies are introduced to minimise optical losses in solar cells. Besides antireflection coatings, the focus within this chapter is placed on solar cell texturisation. First, general considerations on optical effects caused by different spatial frequencies of textures required to achieve a reduced reflectivity and effective light trapping are presented. Then, different maskless texturing technologies are explained, followed by an overview of approaches to realise defined textures. This chapter also deals with the possible increase in the surface recombination rate, which is an unwanted negative effect of texturing solar cell surfaces. There, a literature review is given to sum up potentially occurring effects and to be able to formulate some dos and don'ts. Finally, texturing approaches are rated.

Chapter $\mathbf{4}$ is about the texturing chain developed within this work. Interference lithography is presented as a mastering technology. The stamp replication into polymeric materials is realised using cast moulding or hot embossing. Then, the actual NIL process step is conducted. In this part, the two tools for NIL developed within this work are described. These are namely a conventional NIL tool relying on a planar stamp setup and a Roller-NIL tool. An important issue in NIL is related to the choice of resist materials. In this context different types of solvent-based and solvent-free UV-curing resist materials are investigated. As the last point in this chapter, the plasma etching technology is explained with a focus on PV applications, and the important issue of potentially occurring plasma damages is described. 
In chapter $\mathbf{5}$ the two application examples investigated within this work are explained (the front-side honeycomb texturing and diffractive rear-side gratings). For both applications, the full texturing process chain - consisting of mastering, stamp fabrication, NIL and plasma etching - is described and optical and electrical characterisation results are presented and discussed. For the honeycomb texturing, solar cell results are reported both on small cell areas using the conventional NIL tool as well as large area $\left(125 \times 125 \mathrm{~mm}^{2}\right)$ cells using the Roller-NIL tool. For the realisation of diffractive rear side gratings, the efficiency potential is estimated based on minority carrier lifetimes determined on textured samples in combination with wave optical modelling to determine the photogeneration profiles. 



\section{Wafer based silicon solar cells}

In this chapter, the basic physical properties of silicon will be explained starting from its crystal structure. An emphasis will be placed on its optical characteristics and their implications on solar cells made from silicon. Also, the basics in terms of electrical properties and the principles of solar cells will be explained. The main characteristics of an ideal solar cell are shown, before the main loss mechanisms are introduced. Finally, an overview of established processes for a "standard" silicon solar cell fabrication as well as already mature processes in laboratory environments will be given.

\subsection{Silicon as a solar cell material}

\subsubsection{Crystal structure and implications on optical properties}

In terms of their electrical properties, solid state materials can be divided into three classes: (i) conductors, (ii) insulators and (iii) semiconductors. The principle of solidstate solar cells is based on the electrical properties and their consequences on the optical characteristics of semiconductors. Therefore, the origin of the electrical properties of silicon will be explained briefly.

Crystalline semiconductors can be explained best by assuming a perfect periodic single crystal in which the atoms have some arrangement. In the case of silicon, this arrangement is ordered in the so-called diamond lattice. This type of lattice can be seen as two overlaid face-centred-cubic (fcc) sub-lattices, where one of these sublattices is displaced along the body diagonal of the other one. This displacement is one quarter of the length of the body's diagonal. This lattice structure is shown in Figure 2.1. 


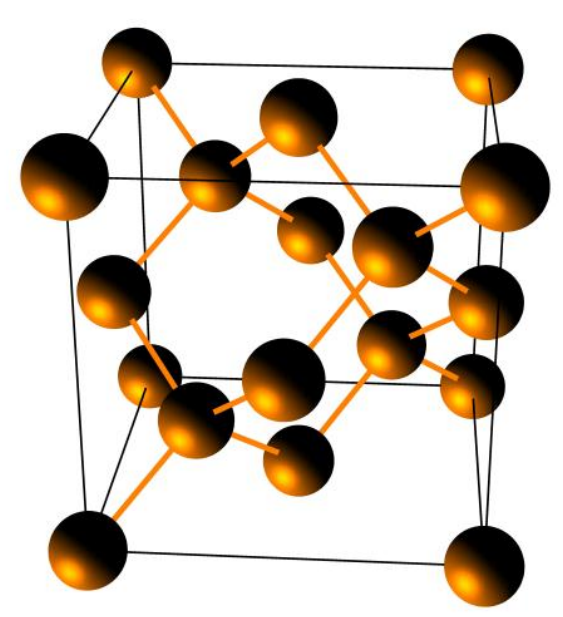

Figure 2.1: Crystal structure of silicon.

For different crystal planes, as shown in Figure 2.2 through this type of lattice, there are different densities of atoms and therefore the atomic spacing varies. This is of importance when it comes to chemical stability of different crystal planes in etching processes (e.g. anisotropic etching in alkaline solutions). Further on, this different atomic spacing for different crystal planes also will have an effect on the quality of electrical surface passivation, since a different amount of free bonds has to be saturated in different planes. A method for defining distinct crystal planes is given by the Miller indices. The following explanation of how to obtain these indices is taken from [8]:

(i) Three Cartesian coordinates with respect to the lattice constant $a^{1}$ are used to describe the axis interceptions in the $(x, y, z)$-directions.

(ii) The reciprocals of these numbers are taken and reduced to the smallest three integers having the same ratio.

(iii) The result is enclosed in parentheses (hkl) as the Miller indices for a single plane.

A convenient extension to this definition is to describe crystal planes in the form of e.g. $\langle 100\rangle$, which holds for a set of equivalent planes ((100), (010) or (001)).

\footnotetext{
${ }^{1}$ The lattice constant of silicon is $5.43 \AA$ [8].
} 

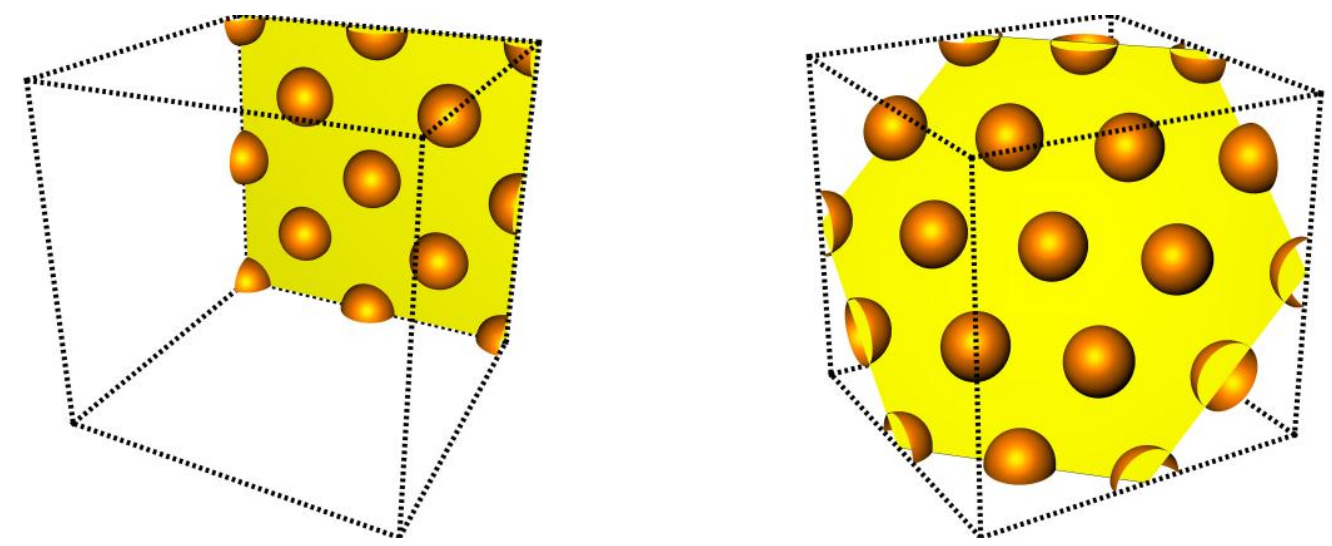

Figure 2.2: Cuts through distinct crystallographic planes of a silicon single crystal. On the left hand side a (100) plane and on the right hand side a (111) plane is visualised. The atomic packing density for the (111) plane is higher than for the (100) plane. For both plots, the cube size is twice the silicon lattice constant of $0.543 \mathrm{~nm}$.

The basic principle of growing silicon single crystals is briefly explained in section 2.3.1. Electrical and some optical properties of solid-state materials are often described by energy bands of bound (valence band) and free (conduction band) charge carriers. While for metals these bands are overlapping (indicating that there is no need for additional energy to generate free carriers), for insulators there is a large energy gap between these bands (high energies are necessary to excite electrons into the conduction band). In the following, the origin of energy bands as well as the filling of them in a silicon semiconductor is explained.

Looking at one isolated atom, discrete energy levels for electrons can be formulated. These energy levels correspond to distinct wave functions and they can be described by the Schrödinger equation. When two atoms are brought together in the angstrom scale, wave functions of electrons can overlap. Extending this to a periodic crystal lattice of silicon atoms, the wave functions of electrons have to fit into a periodic potential implied by the atoms as described by the Schrödinger equation. Solving the Schrödinger equation with Bloch's theorem for the appropriate potential implied by the atoms, energy bands in the three-dimensional momentum space can be found. 


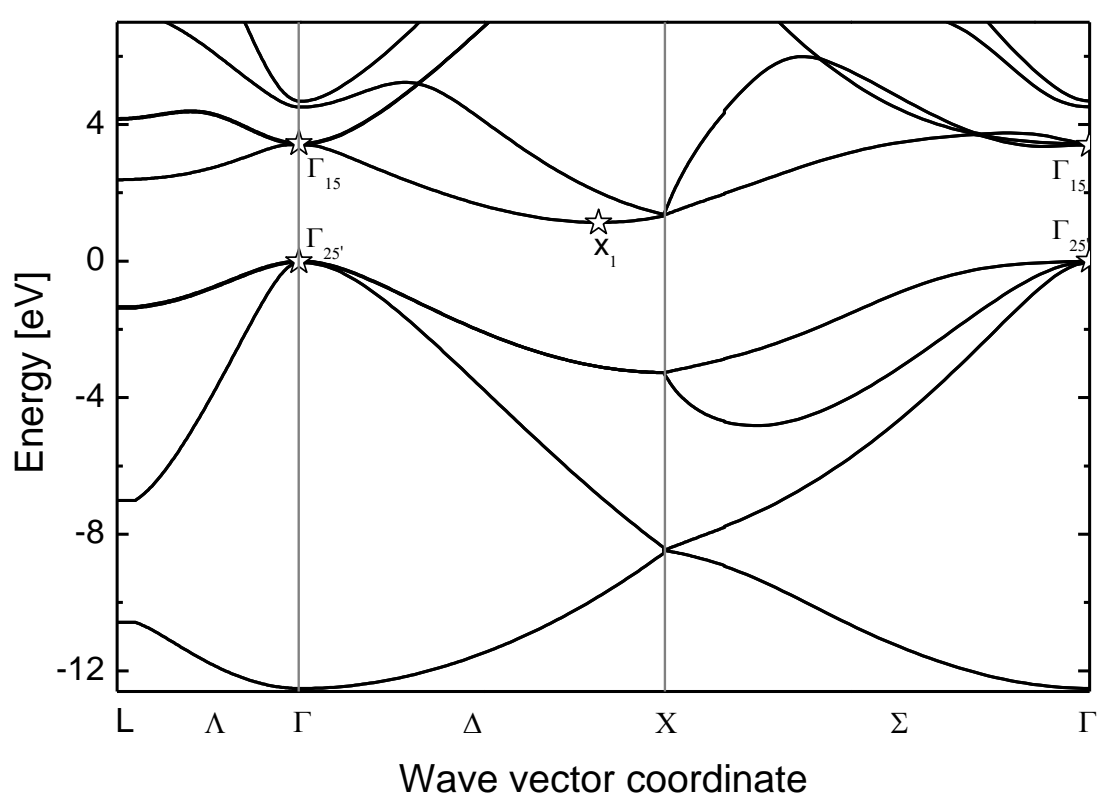

Figure 2.3: Band structure of silicon calculated by a tight binding method [9]. Also shown are selected points in the energy momentum space according to [10].

Besides having implications on the electrical properties, the relation of energy over wave vectors, as shown in Figure 2.3, also determines the optical absorption and emission behaviour of silicon. The bandgap of silicon is indirect; the valence band maximum exists for different momentum of the electron than the conduction band minimum (see Figure 2.3: indirect band gap from $\Gamma_{25}$, to $x_{1}$ on the momentum space axis). For an electron to be excited from the valence band to the conduction band by a photon that has exactly the band gap energy $E_{C}-E_{V}(1.12 \mathrm{eV})$, it must not only absorb the photon but also experience a change in momentum. The change can be induced by the absorption of a phonon. The requirement of simultaneous photon and phonon absorption causes this low energy indirect transition to be less probable than a direct transition and hence the photon absorption is weaker. Direct transitions also exist for silicon. One direct transition is possible from the points $\Gamma_{25}$, to $\Gamma_{15}$, which requires a photon energy of $3.4 \mathrm{eV}$. In Figure 2.4 the wavelength-dependent absorption-coefficient as well as the penetration depth of silicon are shown. Compared to semiconductors with a direct band gap like Gallium Arsenide, the absorption length (or penetration depth) of silicon can be very long. Light with a photon energy of around $E_{\text {Gap }}$ needs to travel centimetres until its intensity is decayed to 1/e. This absorption behaviour of silicon has to be considered when aiming for high-efficiency solar cells; very long path lengths of light have to be achieved in the weakly absorbed range for maximum absorption. Strategies to 
achieve this are explained in section 3.1 and are one major aspect for the work described in chapter 5 .

Up to now, optical absorption caused by the excitation of electron-hole pairs has been described. However, there are other absorption effects like free-carrier absorption (FCA) or lattice absorption. Neither of these contribute positively to the performance of a solar cell, since no extra electron-hole pairs are generated. Instead, thermal energy is given to the lattice in form of phonons. Lattice absorption only takes place for long wavelengths around $10 \mu \mathrm{m}$. FCA takes place in regions of high free-carrier densities such as highly doped regions. Since the extinction coefficient implied by FCA is low [11], its influence in a standard solar cell is not very high. However, when effective light trapping is achieved, weakly absorbed light in the long wavelength range has to cover large distances within the cell and thereby the contribution of the FCA increases [12].

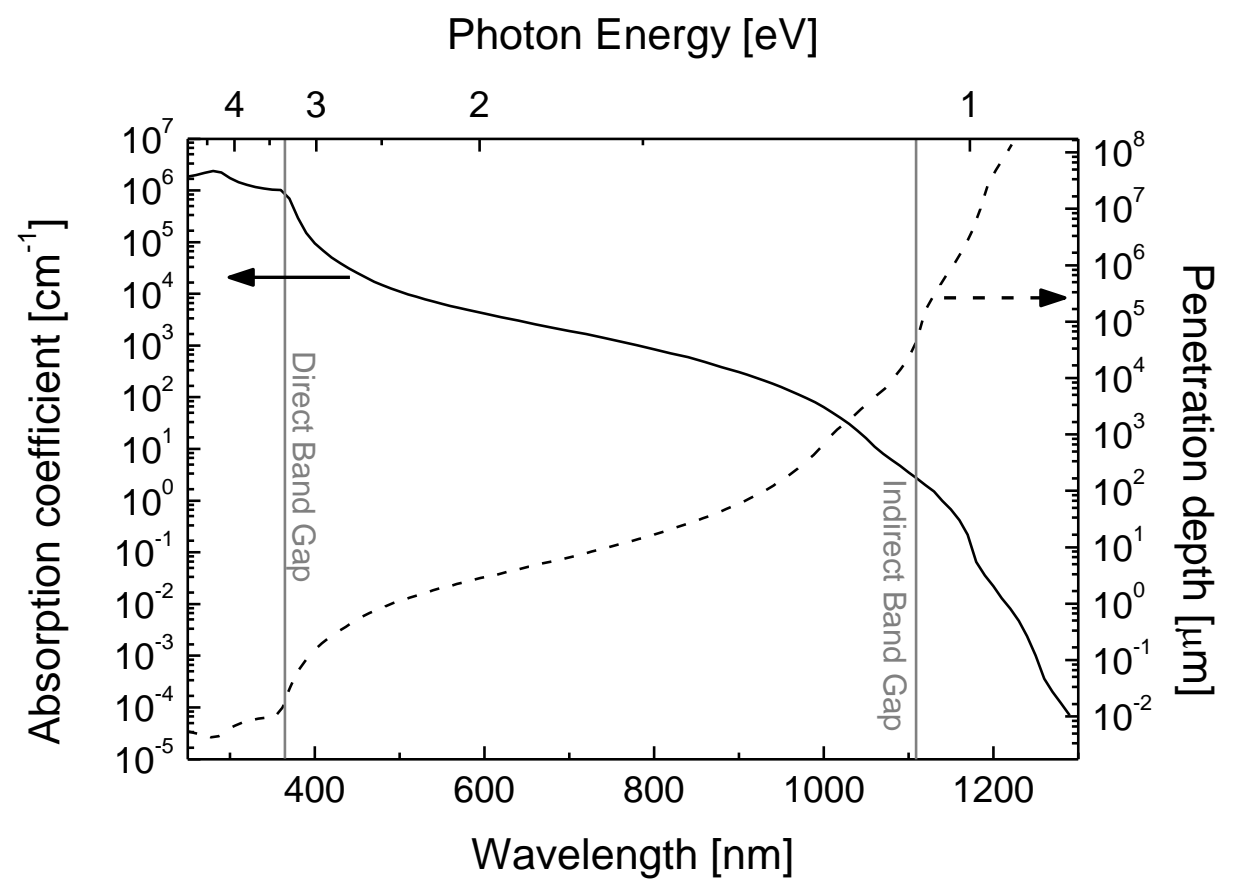

Figure 2.4: Absorption coefficient and penetration depth of silicon at $300 \mathrm{~K}$. Data is taken from [13].

\subsection{Electrical properties}

The energy-momentum relation shown in Figure 2.3 also sets implications on the electrical properties of silicon. In addition to this relation, allowed energy levels as well as a density of these states $D(E)$ for electrons and holes can also be calculated. The conductivity of silicon is mainly determined by the number of free charge carriers. Silicon atoms in a perfect single crystal have four electrons in their outer atomic shell, which are bound at absolute zero temperature. This implies that the electrons fill the 
valence band completely and none are in the conduction band. In this case, there are no free charge carriers to provide any conductivity. Increasing the temperature, some electrons are lifted up into the conduction band leaving behind a hole ${ }^{2}$. This temperature-dependent effect is described by Fermi-Dirac statistics, which also introduce the important Fermi energy level $E_{F}$. At absolute zero, all energy levels are filled up to $E_{F}$.

The quantity of free electrons $n$ in allowed energy states can be described as in Equation (2.1). $D(E)$ is the density of states and $F(E)$ is the Fermi-Dirac distribution, which describes the probability of occupation of these states. The integration only occurs above the conduction band energy $E_{C}$, since free electrons can only be found there. Holes in the valence band can be treated analogously.

$$
n=\int_{E_{C}}^{\infty} D(E) F(E) d E
$$

Using Boltzmann statistics as a simplification of the Fermi-Dirac statistics, the following equations for free electrons and holes in thermal equilibrium can be derived:

$$
\begin{aligned}
& n=N_{C} e^{\left(E_{F}-E_{C}\right) / k T} . \\
& p=N_{V} e^{\left(E_{V}-E_{F}\right) / k T} .
\end{aligned}
$$

Here, $N_{C}$ and $N_{V}$ are effective densities of states for electrons in the conduction band and for holes in the valence band respectively. The intrinisic charge carrier density $n_{i}$ can be related to $n$ and $p$ by the Law of Mass Action

$$
n_{i}^{2}=n p=N_{C} N_{V} e^{\left(E_{F}-E_{C}+E_{V}-E_{F}\right) / k T}=N_{C} N_{V} e^{\left(E_{G a p}\right) / k T} .
$$

A long list of sources concerning the determination of $n_{i}$ can be found in the literature. This number, which corresponds to both the hole as well as electron concentration in undoped silicon is about $1 \cdot 10^{10} \mathrm{~cm}^{-3}[14]$.

By introducing so-called dopants, which are atoms acting as charge donors or acceptors, the value either $n$ or $p$ assume in thermal equilibrium can be changed. Typical doping densities in silicon solar cells are around $10^{16}$ to $10^{20}$ atoms per $\mathrm{cm}^{3}$. Since the relation $n_{i}^{2}=n \cdot p$ still holds for doped silicon, $n_{i}$ is constant, and the number of free carriers introduced by the dopant is some orders of magnitude higher than the intrinsic carrier densities, the opposite charge type to the dopant's is diminished. For example, in $n$-doped (also called $n$-type) silicon if $n$ equals $10^{16} \mathrm{~cm}^{-3}$,

\footnotetext{
${ }^{2}$ Hole: The equivalent to an electron, which carries a positive charge. However effective masses and thus mobilities are different to the ones of electrons.
} 
$p$ must equal $\left.10^{4} \mathrm{~cm}^{-3}\right)$. In this case, electrons would be denoted majority carriers and holes minority carriers, with the opposite being the case for $p$-doped silicon.

When a semiconductor is illuminated, photons of a higher energy than the band gap energy $E_{\text {Gap }}$ generate additional free electron-hole pairs. Thus the relation $n_{i}^{2}=n \cdot p$ is not valid any more. This also implies that there is no longer a single Fermi level $E_{F}$, but separate so-called Quasi-Fermi-levels for electrons $\left(E_{F, n}\right)$ and holes $\left(E_{F, p}\right)$. This leads to

$$
\begin{array}{ll}
n=N_{C} e^{\left(E_{F, n}-E_{C}\right) / k T} & \text { and } \\
p=N_{V} e^{\left(E_{V}-E_{F, p}\right) / k T} &
\end{array}
$$

under illumination.

\subsubsection{Generation and Recombination}

When electrons are steadily excited to states in the conduction band with a generation rate $G\left(\left[\mathrm{~cm}^{-3} \mathrm{~s}^{-1}\right]\right)$ (either thermally or by optical absorption) and no charge carriers are extracted out of the semiconductor (meaning that $\nabla J=0$ ), an equilibrium has to be reached. This is described by the continuity equations as shown exemplarily in equation (2.7). The following equations are just shown for electrons, but are also transferable to holes (with some changes in the mathematical sign).

$$
\frac{\partial n}{\partial t}=G-R+\frac{1}{q} \nabla J_{n}
$$

A recombination rate $R\left(\left[\mathrm{~cm}^{-3} \mathrm{~s}^{-1}\right]\right)$ is introduced, which balances the generation rate in the scenario described above. Using this recombination rate and the excess carrier density $\Delta n$, a lifetime $\tau$ of generated charge carriers can by defined via

$$
\tau=\frac{\Delta n}{R}
$$

There are several possibilities for excited charge carriers to recombine. A description of possible recombination mechanisms as well as characterisation methods is given in [15]. An effective lifetime $\tau_{\text {eff }}$ can be calculated considering the mechanisms of radiative, Auger, Shockley-Read-Hall $(\mathrm{SRH})$ or surface recombination, which are indicated in the indices below, via

$$
\frac{1}{\tau_{\text {eff }}}=\frac{1}{\tau_{\text {radiative }}}+\frac{1}{\tau_{\text {Auger }}}+\frac{1}{\tau_{S R H}}+\frac{1}{\tau_{\text {surface }}}
$$




\section{Recombination in the bulk}

In the following, some recombination mechanisms active in the silicon bulk are explained briefly. In Figure 2.5 the mechanisms of radiative recombination, Auger recombination and recombination via defect states within the band gap (ShockleyRead-Hall recombination) are visualised.
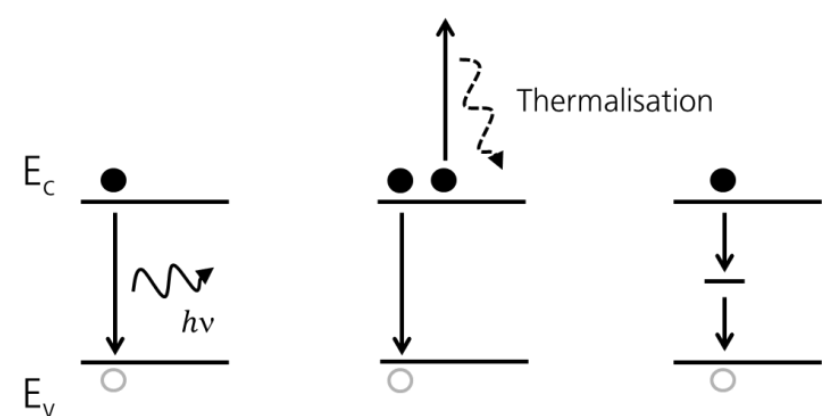

Figure 2.5: Sketch of radiative recombination, Auger recombination and recombination via defect states (from left to right).

The recombination process of excited charge carriers has to obey energy conservation; therefore, the sketched variations of recombination processes mainly vary in the way the "lost" energy is converted. In the case of radiative recombination, this is very easy, as it can be seen as the inverse process of optical excitation. Thus, a photon is emitted, having the band gap energy $E_{\text {Gap }}$. In the case of the Auger recombination, a third charge carrier is involved in the recombination process. The energy put into the photon emission for the radiative recombination process is given to this third carrier, which is excited to a higher energy in the conduction band and later thermalizes to its initial energy. These two processes of radiative as well as Auger recombination are intrinsic mechanisms that cannot be avoided.

The recombination via defect states, also called Shockley-Read-Hall (SRH) recombination, is related to material quality issues. The defect density and particularly the energetic level of the defect states within the band gap are crucial determinants of SRH recombination rates $[16,17]$. The most critical defect materials that lead to high SRH recombination are metals such as Copper, Iron or Gold, which create defect states close to the middle of the band gap. Again, here the energy lost by recombination is set free in the form of thermal energy or photons (defect luminescence) [18].

\section{Surface recombination}

Band calculations and most considerations about electrical and optical properties of silicon are based on the assumption of an infinitely extended single crystal. It therefore seems clear that an abrupt end of a periodic crystal goes in hand with the 
end of a perfect band gap. At the surface, defect states are distributed continuously all over the band gap. A recombination rate at the surface $R_{\text {Surf }}\left(\left[\mathrm{cm}^{-2} \mathrm{~s}^{-1}\right]\right)$ can be calculated similarly to the formalism introduced by Shockley, Read and Hall. The dimension of this recombination rate indicates that this recombination scales with the area, which will be of importance when it comes to texturing the surface. In the SRH recombination single distinct defect levels are considered, but since there is a continuum of states for surface recombination, an integration over the band gap is introduced [19]:

$$
R_{\text {Surf }}=\left(n_{s} p_{s}-n_{i}^{2}\right) \int_{E_{V}}^{E_{C}} \frac{v_{t h} D_{i t}\left(E_{t}\right) d E_{t}}{\left(n_{s}+n_{1}\left(E_{t}\right)\right) \sigma_{p}^{-1}\left(E_{t}\right)+\left(p_{s}+p_{1}\left(E_{t}\right)\right) \sigma_{n}^{-1}\left(E_{t}\right)},
$$

with $v_{t h}$ being the thermal velocity, $D_{i t}$ the interface defect density and $E_{t}$ the defect energy level. $n_{s}$ and $p_{s}$ are the electron and hole concentrations at the surface and $\sigma_{p}$ and $\sigma_{n}$ are the capture cross section for holes and electrons. $n_{1}$ and $p_{1}$ are the Shockley-Read-Hall electron and hole densities, which are defined as

$$
n_{1}(E)=N_{C} e^{\frac{E_{t}-E_{C}}{k T}} \quad \text { and } \quad p_{1}(E)=N_{V} e^{\frac{E_{C}-E_{G a p}-E_{t}}{k T}} .
$$

To reduce the complexity of this description, a recombination process over one single defect level can be assumed. An approximation for $R_{\text {Surf }}$ in the case of low injection is given in [8], where also a surface recombination $S$ is introduced, which has dimensions of centimetres per second:

$$
R_{\text {Surf }} \cong \sigma_{p} v_{t h} D_{i t} \Delta n \equiv S \Delta n
$$

These relations can be used to explain two strategies for minimizing surface recombination [20]:

(i) A minimization of the defect density $D_{i t}$ : This can be achieved by a chemical saturation of dangling bonds at the silicon surface (e.g. by a thermal silicon oxide).

(ii) Minimizing the minority carrier density at the surface since the recombination process requires both types of charge carriers: This can be achieved by a so-called field effect passivation, by which one type of carriers is repelled from the surface e.g. by fixed charges incorporated in a dielectric layer deposited on the silicon surface (e.g. positive charges in a silicon nitride layer). 


\subsubsection{Ideal solar cell}

A simplified $p$-type solar cell is sketched in Figure 2.6. It consists of a $p$-doped semiconductor base (also called $p$-type base, with a thickness of 150 to $300 \mu \mathrm{m}$ ) and a thin highly doped region ( $n$-type, with a thickness of 0.2 to $2 \mu \mathrm{m}$ ) at the front, which is called the emitter. When this semiconductor device is illuminated, free charge carriers are generated by the absorption of photons with energies higher than $E_{\text {Gap }}$. Within the electric field of the $p n$-junction, these optically excited electron-hole pairs can be separated and then extracted via contacts at the front and back side, which are connected to an electrical circuit to perform work.

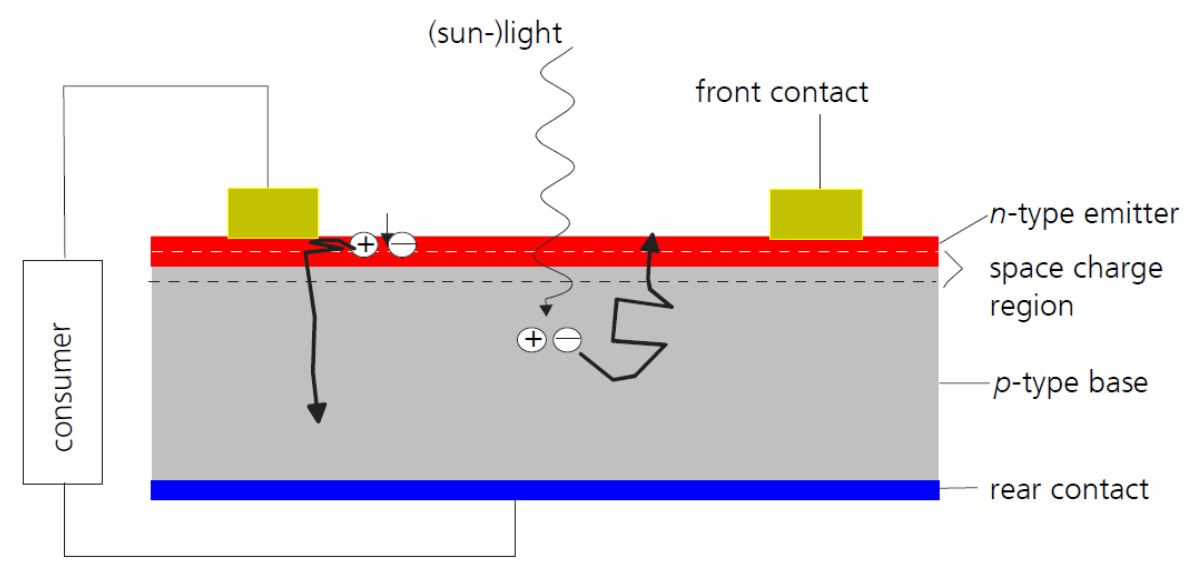

Figure 2.6: Sketch of a solar cell comprising the basic elements of an $n$ - and a p-type semiconductor (emitter and base respectively) and front and rear contact. Photo-generated electron-hole pairs are separated by the field in the space charge region between the differently doped regions. This graphic is taken from [21].

The easiest way to describe the behaviour of this solar cell is based on the one-diode equation introduced by Shockley:

$$
J(V)=J_{0}\left(e^{\frac{q V}{n k T}}-1\right)-J_{p h},
$$

where $J_{0}$ is the dark saturation current (describing the recombination behaviour of emitter and bulk), $n$ is the diode ideality factor ${ }^{3}, k$ is Boltzmann's constant, $q$ is the elementary charge and $T$ the solar cell temperature. Additionally, $J_{p h}$ is the photogenerated current. $J(V)$ is plotted in Figure 2.7, demonstrating the most important solar cell parameters, which are namely the short circuit current $J_{s c}$, the open circuit voltage $V_{o c}$, and the fill factor $F F$. These parameters can be used to calculate the

\footnotetext{
${ }^{3}$ The ideality factor is related to recombination effects. For an ideal $p n$-junction the ideality factor equals one, when introducing e.g. recombination in the space charge region or Auger recombination its value is typically between one and two.
} 
maximum power that can be extracted from the solar cell at so-called maximum power point (MPP) conditions. A more realistic model is given by the two-diode equation, where additionally a recombination in the space charge region as well as series and parallel resistances can be considered [22].

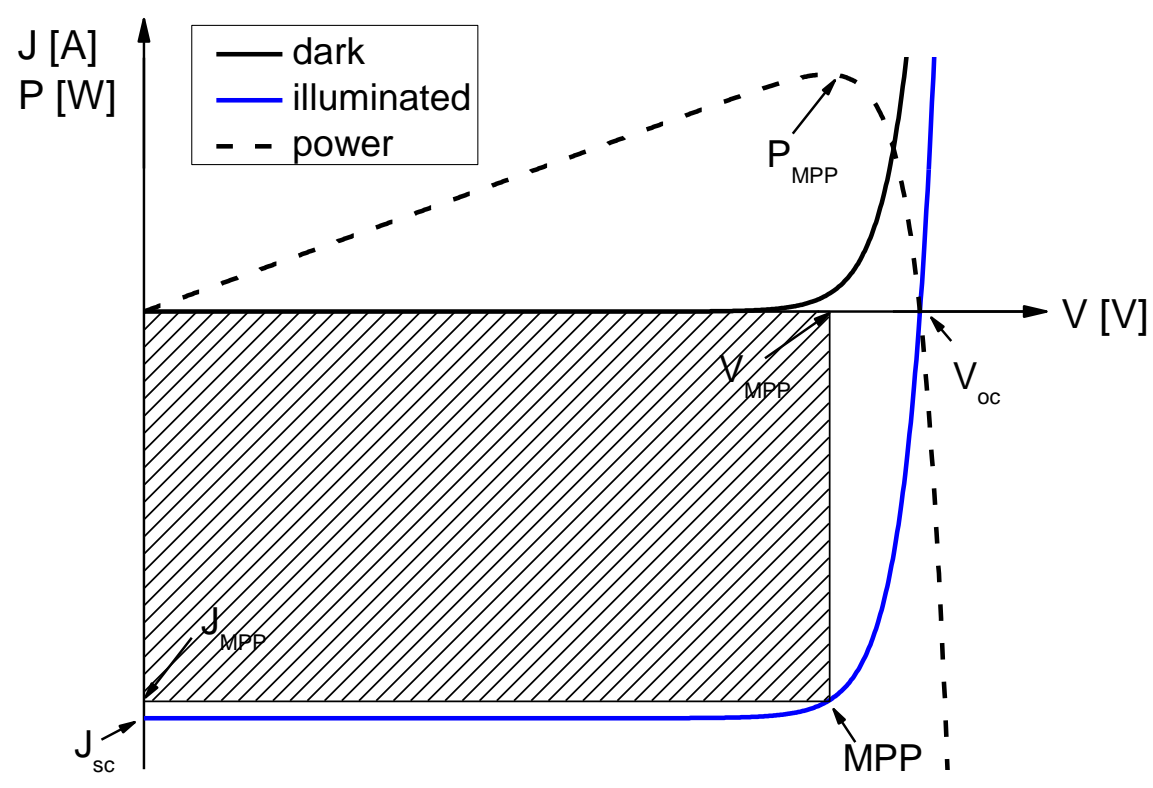

Figure 2.7: Characteristic current-voltage relation of a solar cell as calculated by the one-diode equation. Additionally, the resulting extractable power is shown and the maximum-power-point (MPP) is visualized.

As can be seen, the maximum power extractable from a solar cell is not equal to the product of $J_{s c}$ and $V_{o c}$, but to the product of $J$ and $V$ at MPP conditions. This discrepancy is described by the fill factor via

$$
F F=\frac{J_{M P P} V_{M P P}}{J_{S c} V_{o c}}
$$

Further on, the efficiency $\eta$ of a solar cell can be calculated with respect to the irradiance by

$$
\eta=\frac{P_{M P P}}{P_{\text {Irradiance }}}=\frac{F F J_{S c} V_{o c}}{P_{\text {Irradiance }}} .
$$

The theoretical limit for the conversion efficiency of a single junction solar cell under AM1.5g illumination as formulated by Shockley and Queisser is $33 \%[23,24]$. This is calculated assuming only radiative recombination and no concentration of sun light is considered. The maximum efficiency is dependent on the band gap energy of the semiconductor material, which in this special case is around $1.3 \mathrm{eV}$ (which is close to 
that of silicon). Photons of energies below $E_{\text {Gap }}$ are not capable of generating electron-hole pairs. Photons of higher energies than $E_{\text {Gap }}$ can only excite one electron-hole pair and their energy exceeding $E_{\text {Gap }}$ is transferred into heat. Models to calculate a limit of efficiency have been refined, especially for silicon solar cells, additionally considering Auger recombination, leading to a limit of $29 \%$ both for $n$ and $p$-type silicon [25]. The wafer thickness was also taken into account in this study and interestingly this limit of efficiency is reached for a $90 \mu \mathrm{m}$ thick cell. This shows that it is beneficial for the electrical properties of the cell that it should be very thin (bulk recombination is minimized). However, a very effective photon management must then be achieved to maintain high absorption over the solar spectrum. Therefore, concepts like the one presented in section 5.2, relying on diffractive effects, could contribute to approaching this efficiency limit.

\subsubsection{Real solar cell}

There are several effects reducing the maximum efficiency in a silicon solar cell. Beside the band gap induced losses as described before, loss mechanisms are either of electrical or optical origin. The electrical effects of recombination have already been explained briefly. One can divide between intrinsic effects (radiative and Auger recombination) and the extrinsic effect of the SRH-recombination, which is defined by the material quality. The intrinsic effects themselves cannot be avoided, although steps can be taken to reduce them, e.g. by avoiding too high doping concentrations in the emitter formation, Auger recombination can be minimised. Other electrical effects reducing the efficiency of a solar cell concern the series and parallel resistances. The series resistance should be minimised to reduce Ohmic losses. The parallel resistance corresponds to a shunting of emitter and base and thus this resistance should be as high as possible.

Four aspects leading to optical losses are sketched in Figure 2.8 for a standard silicon solar cell, which are namely: reflection and escape (or transmission) losses, shading and parasitic absorption. 


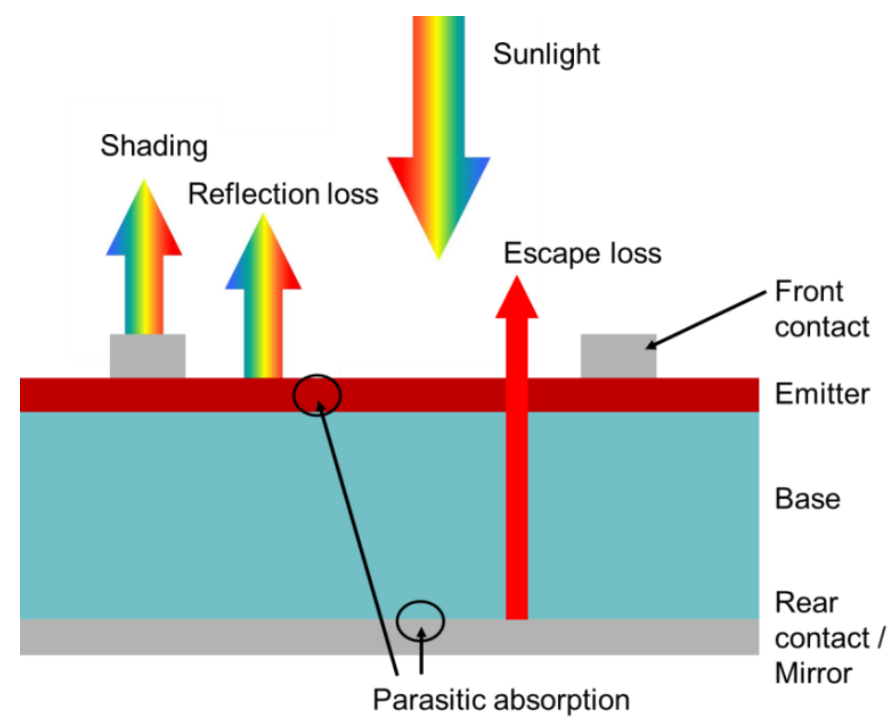

Figure 2.8: Sketch of four origins of optical losses in a standard silicon solar cell.

Shading occurs due to the metal coverage on the front side of the solar cell. For a standard solar cell, the fraction of metal coverage is about $2-7 \%$ [26]. This shading is avoided in back contact solar cells, in which both emitter and base contacts are placed on the rear [27]. Parasitic absorption can occur in different places in the solar cell. The main mechanism is absorption in the metal contact / mirror, but also freecarrier absorption (FCA) occurs. FCA is dependent on the carrier density and therefore mainly occurs in highly doped regions like the emitter. For most of the solar cells produced today, the rear contact is printed directly on the silicon. During a firing step, a so-called aluminium Back-Surface-Field (BSF) is formed (described in section 2.3.2). The internal reflectivity of such an interface is only about $65 \%$ [28]. Thus, considerable parasitic absorption occurs at this interface. Both reflection and escape losses can be minimized by photon management structures. Beside these surface textures, antireflection layers are also important measures to minimize reflection losses.

A minimization of these optical losses leads to a higher generation within the solar cell. Therefore, on first sight $J_{s c}$ should benefit especially from these efforts. In the following two equations it is shown that not only $J_{s c}$ is increased by an enhanced absorption, but also $V_{o c}$ and the ideal fill factor:

$$
V_{o c}=\frac{k T}{q} \ln \left(\frac{J_{s c}}{J_{0}}+1\right) \quad \text { and }
$$




$$
F F=\frac{V_{o c} / \frac{k T}{q}-\ln \left(V_{o c} / \frac{k T}{q}-0.72\right)}{V_{o c} / \frac{k T}{q}+1}
$$

Equation (2.16) is the solution of the one-diode equation for $V_{o c}$. Equation (2.17) to calculate the ideal fill factor is based on empirical findings and is shown for an ideality factor of one [10]. In Figure 2.9 graphs for both equations as functions of $J_{s c}$ are plotted. In the following some limitations for $V_{o c}, F F$ and $J_{s c}$ are listed.

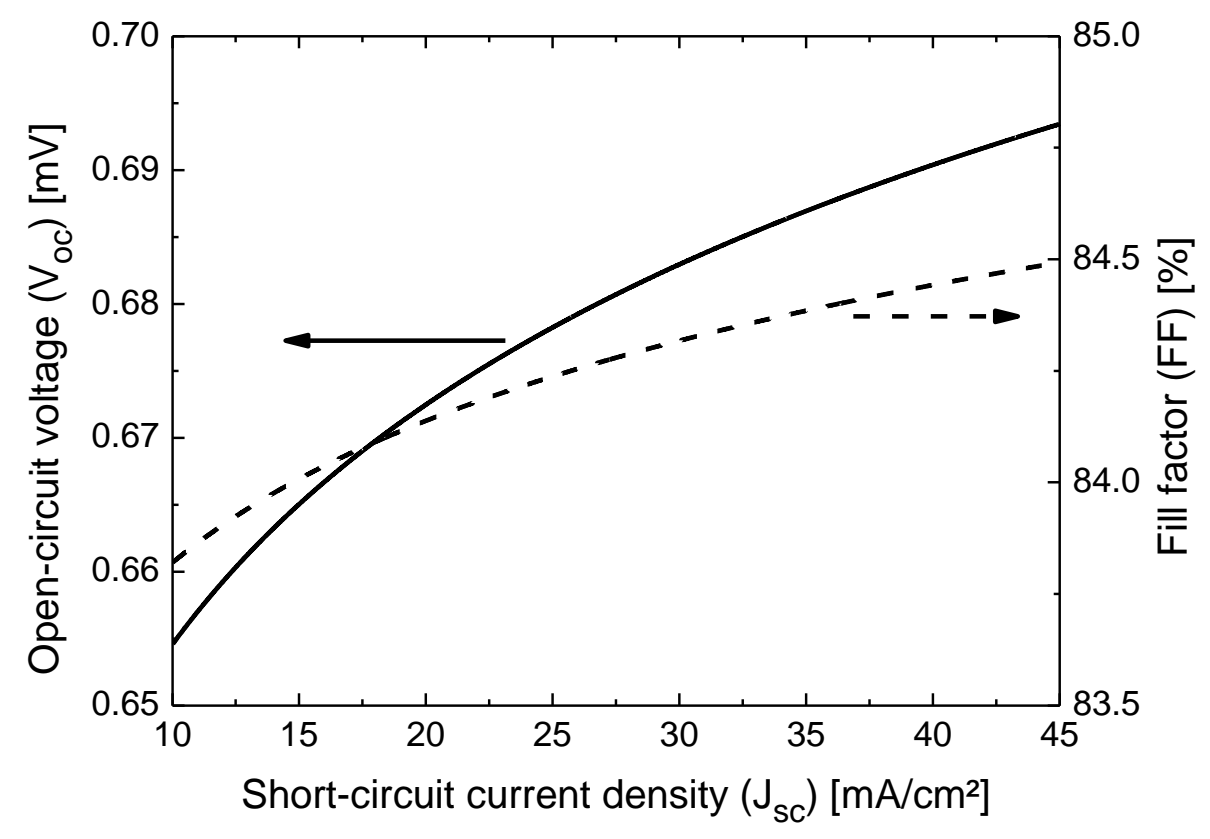

Figure 2.9: Calculated limits of $V_{o c}$ and FF using equations (2.16) and (2.17). Assumptions made in these graphs are a saturation current density of $100 \mathrm{fA} / \mathrm{cm}^{2}$ and in case of $F F$ an ideality factor of one.

\section{Limits to $F F, V_{o c}$ and $J_{s c}$}

- The fill factor of a solar cell reaches its maximum for zero series resistance (e.g. contact resistances), an infinitively large parallel resistance (this concerns shunts) and minimal dark saturation current densities. Practical considerations lead to a maximum fill factor of around $85 \%$ [10].

- The limit of $V_{o c}$ is not defined by $E_{G a p}(1.12 \mathrm{eV}$ for silicon), but by the splitting of the quasi-Fermi levels [29]. Very high values for $V_{o c}$ can be achieved for hetero-junction cells; values of up to $747 \mathrm{mV}$ have been reported [30].

- Integrating all photons in the AM1.5g spectrum with boundaries relevant for silicon solar cells (energies above $1.12 \mathrm{eV}$ ), one obtains a maximum for the achievable short-circuit current density $j_{s c}$ of $43.8 \mathrm{~mA} / \mathrm{cm}^{2}$. Very high values 
for $j_{s c}$ on FZ c-Si high-efficiency solar cells of $42.2 \mathrm{~mA} / \mathrm{cm}^{2}$ were already measured [31].

The highest efficiencies measured on silicon solar cells without concentration are $24.7 \%$ for monocrystalline [31] and $20.3 \%$ for multicrystalline silicon [5] (these values were corrected to $25 \%$ and $20.4 \%$ respectively [32] for the corrected AM1.5g spectrum in 2008 [33]). The difference between the efficiencies for these materials is mainly related to material quality (mainly through minority carrier lifetime), but also on the quality of producible front-side textures. For both of these record cells, very high external quantum efficiencies were achieved, these being about $96 \%$ and $86 \%$ respectively for the incoming photons in the relevant spectral range. One reason for this very efficient photon harvesting is that these solar cells featured photolithographically defined front side textures. Existing techniques to enable a designed patterning are either too complex and costly or do not deliver necessary resolutions to transfer these high efficiency features to industrial production. The present work concentrates on this discrepancy. To understand the requirements for introducing novel processes into solar cell fabrication, the fabrication steps of a typical industrially manufactured solar cell are explained in the following.

\subsection{State of the art of silicon solar cell fabrication}

A standard process flow in industrial fabrication of silicon solar cells contains the following steps:

Silicon purification, forming of ingots or bricks, wafering, removal of saw damages and texturisation, emitter diffusion, deposition of an antireflection coating (ARC), screen printing of a metal paste forming a grid on the front side and a fully coating on the backside, a firing step for the contact formation and finally the edge isolation.

In the following, these steps will be explained briefly for processes as used for the fabrication of a screen printed $p$-type silicon solar cell with an aluminium BSF; this still being the industrial standard (with few exceptions). In the last part of this section, some concepts to increase cell efficiencies and technologies, still maintaining feasibility for an industrial realisation, are presented.

\subsubsection{From sand to mono- or multicrystalline wafers}

Silicon is the second most abundant and probably the best-investigated material on earth, being the standard material not only for semiconductor applications but also for micro-electro-mechanical systems (MEMS) [34]. There are several types of silicon, which can mainly be distinguished by the grade of material purity. As the first step, 
metallurgical grade silicon is obtained by chemically reducing high purity quartzite $\left(\mathrm{SiO}_{2}\right)$ with carbon in a furnace at temperatures around $1800{ }^{\circ} \mathrm{C}$. This metallurgical grade silicon already has a purity of around $98 \%$ [8]. For the realisation of electronic grade silicon, which is necessary for the integrated circuit industry, metallurgical grade silicon is first converted into trichlorsilane and then reduced by hydrogen to form highly pure silicon. Since the resulting quality of electronic grade silicon is even higher than is required for solar cells (in terms of a cost-benefit ratio), there are different approaches to produce slightly less pure solar grade silicon [35].

Multicrystalline silicon is fabricated by melting relatively pure silicon in a crucible e.g. made of quartz and assuring a controlled solidification. Already in this stage a background doping of the silicon is achieved by introducing dopants like boron or phosphorus into the melt (examples of $p$ - and $n$-type doping respectively). The solidification has to proceed slowly from the bottom to the top of the crucible. This controlled solidification allows impurities and contaminations to migrate along the solidification front and also induces columnar growth of crystal grains. The reason for the migration of contaminations is that the liquid phase of silicon has a higher solubility of metal impurities. This leads to an increased material quality due to the controlled solidification process in mid regions and a lower quality of the silicon in the top of the ingot or the so-called cap layer, which is therefore removed before the wafering process.

The Czochalski growing technique allows the production of monocrystalline silicon (Cz silicon). A seed crystal is inserted into a silicon melt and is slowly withdrawn while being rotated. Again, dopants are introduced into the melt to achieve a background doping. Even a higher material quality can be obtained using the float zone process, where a seed crystal is placed below a polycrystalline rod and a small melting zone is moved upwards through the rod starting at the very bottom (FZ silicon). The small melting zone is realised by a radio-frequency heater and again one makes use of different segregation coefficients of impurities in the solid and liquid phase. However, FZ material is mainly used in high-efficiency laboratory scale processing. The industrial standard for monocrystalline silicon solar cells is Cz material. In Figure 1.1 it was shown that the PV market is dominated by wafer based silicon solar cells. Within the last years, the share between these two materials was dominated by multicrystalline silicon (in $201157 \%$ of all fabricated solar cells were made of this material; $30.9 \%$ were made of monocrystalline silicon).

After growing the crystalline material, the wafering has to be realised. The most common technique is wire sawing. Nowadays, wafers fabricated by wire sawing can be as thin as $160 \mu \mathrm{m}$ in industrial fabrication [36]. However, since there is considerable kerf-loss of up to more than $50 \%$ of the expensive silicon due to the sawing process, there are several techniques aiming for a reduction of these losses. 
One possibility is to directly form wafers out of the silicon melt as done in ribbon sheet technologies [37]. Another rather exotic but interesting approach is based on implanting hydrogen in a defined depth in an ingot and using this layer to cleave out a wafer [4]. This results in a near zero kerf-loss for the wafering process; however, there are restrictions concerning feasible crystal orientations, leading to drawbacks in the texturing processes later on.

\subsubsection{From wafers to solar cells}

Starting with homogenously doped (most often $p$-type) silicon wafers, the process chain for solar cell fabrication follows. At first, saw damages have to be removed in an etching process. This is typically combined with a texture etching process. For monocrystalline silicon, a wet-chemical treatment in an alkaline solution like potassium hydroxide $(\mathrm{KOH})$ is applied. This leads to pyramidally textured surfaces with excellent optical properties, when using wafers with (100) crystal orientations on their surface $[38,39]$. Multicrystalline silicon is textured in acidic aqueous solutions based on hydrofluoric acid (HF) and nitric acid $\left(\mathrm{HNO}_{3}\right)$ [40]. This etching recipe, namely an isotexture, allows isotropic etching independent of crystal orientations; hence it is advantageous for multicrystalline silicon. It is beneficial to apply the texture etching process with saw damages still on the substrate's surface, because etching reactions preferentially start at these damaged areas, leading to optically more effective textures [41]. However, the isotexture does not lead to an optical quality which is comparable to pyramidally textured surfaces (a detailed description of texturing processes and achievable results is given in section 3.1.2). The alternative wafering technologies mentioned above (ribbon sheet or implanting of a cleaving layer) suffer from inefficient texturing capabilities. In case of ribbon sheet there is no saw damage to allow an efficient acidic texturing [42] and in case of the technology based on implanting a cleaving layer, the (111) crystal orientated surface does not allow an efficient alkaline texturing. Therefore, the process chain presented in this work could also be of interest for these substrate types.

After the texturing process the emitter diffusion follows. For $p$-doped silicon, a donor (most often phosphorus) is driven into the silicon substrate in a diffusion furnace. At high temperatures between $800{ }^{\circ} \mathrm{C}$ and $1000^{\circ} \mathrm{C}$ a carrier gas is pumped through liquid phosphorus oxychloride $\left(\mathrm{POCl}_{3}\right)$ and introduced into the furnace and phosphorus diffuses into silicon according to Fick's laws [43]. When not applying a diffusion barrier, a phosphorus doped layer is formed all around the wafer. During the diffusion a phosphorus-silicate-glass layer (PSG) is built, which has to be removed afterwards in an etching step (mostly wet-chemically using HF).

After the formation of the $p n$-junction the silicon nitride $\left(\mathrm{Si}_{x} \mathrm{~N}_{y}\right)$ antireflection coating is applied on the front side via plasma enhanced chemical vapour deposition (PECVD) 
or sputtering. Next the front and back side are coated with a metal paste by screen printing. While on the front side a grid is applied using a paste containing silver, the backside is coated fully with an aluminium paste. Solvents of this paste are driven out and organic binders are removed in heating steps. In a following firing step at about $800{ }^{\circ} \mathrm{C}$ the contact formation on the front side takes place [44]. There, a glass frit, which is also contained in the paste, penetrates the $\mathrm{Si}_{\mathrm{x}} \mathrm{N}_{\mathrm{y}}$ layer and allows the contacting of the emitter. During this high temperature step on the back side, both the contact formation and the formation of a $p^{+}$layer, the so-called back surface field (BSF), takes place. This consists in building an alloy of silicon and aluminium thus overcompensating the phosphorus doping from the emitter diffusion by a $p$-doping with aluminium. This BSF acts as an electric shield repelling minority carriers from the back surface and thus reduces the effective surface recombination velocity at this interface. After this process flow the emitter is still connected with the p-contact on the back side via the edges of the wafer. This shunt is eliminated by an etching step either by wet-chemical (conducted already after the ARC deposition) or plasma etching, or a laser edge isolation process.

This standard process chain is summarised in Figure 2.10, where the resulting solar cell architecture is also sketched. At the moment the biggest part of industrially fabricated solar cells is made of this structure. Typical efficiencies on c-Si and mc-Si material range from 17 to $19 \%$ and 15 to $18 \%$ respectively. This very easily producible cell concept has drawbacks concerning both electrical and optical properties. In the following section, some drawbacks as well as measures to overcome the thereby implied limitations are explained.
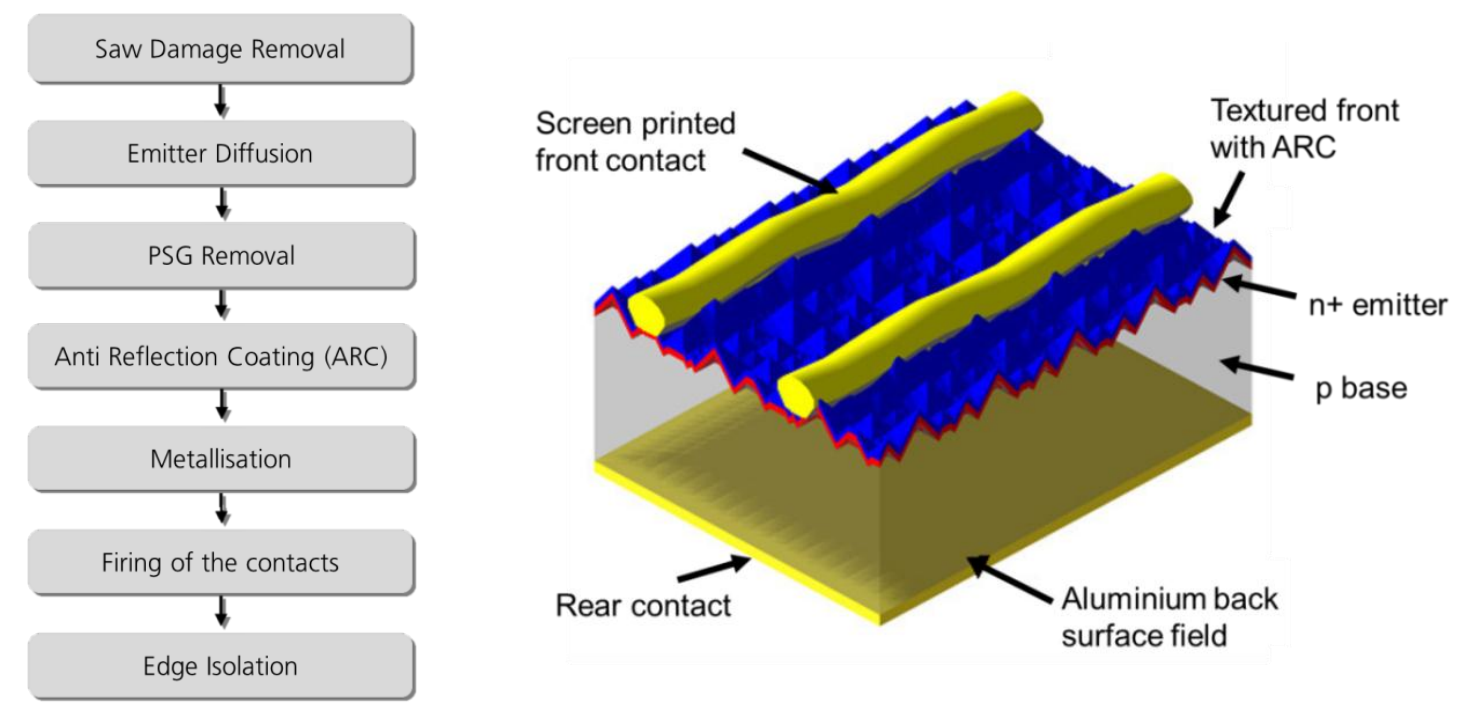

Figure 2.10: Typical process flow of a standard screen printed solar cell with an aluminium back surface field. On the right hand side the resulting cell structure is visualised schematically. Graphic modified from [44]. 


\subsubsection{Laboratory scale processes on the verge of an industrial realisation}

On the laboratory scale, there are plenty of measures to overcome the limitations described above, while still maintaining this cell architecture in principle (solar cells with a diffused front emitter, front and back side being contacted) [28]. Here, some of these features are presented, which are realised by already rather mature technologies and thereby are close to an industrial realisation. The features and processes listed in the following are not meant to be a complete overview but a selection of concepts, which are related to the developments described in this work.

One feature to increase the efficiency of a solar cell is the introduction of a dielectric passivation layer on the rear side as known for the PERC (passivated emitter and rear cell) concept. The benefit arising from a dielectric passivation layer is on the one hand the electrical effect of a reduced surface recombination velocity and on the other hand an optical effect of enhanced reflection properties compared to a back side with an aluminium BSF [45]. Dielectric passivation layers will become more and more important for decreasing cell thicknesses, since there the influence of surface passivation will gain importance and also a wafer bowing as known for the aluminium BSF approach can be avoided [28].

The introduction of a passivation layer on the back side will be of importance when it comes to the realisation of light trapping concepts, in which high internal reflection properties are extremely important. This is visualised in Figure 2.11 by internal quantum efficiencies using a simplified model of a pyramidally textured highefficiency solar cell calculated with PC1D [46]. In the long wavelength range, light is only weakly absorbed and thus travels several times through the $200 \mu \mathrm{m}$ thick cell. For every time light impinges on the back side, a part of it is absorbed there depending on the refractive indices and the optical quality of this interface. Various internal reflectivities for the back side are considered ranging from $65 \%$ to $95 \%$, and specular reflection behaviour is assumed. Internal reflection parameters are oriented to the ones published in $[45,47]$. While the back side reflectivity of $65 \%$ roughly corresponds to the one obtained for an aluminium BSF, the reflection of $95 \%$ is in the range of what is possible for a dielectric passivation layer with local contacts [28]. Additionally, the resulting short-circuit current densities for a single layer ARC on a random pyramid texture are given in the legend of Figure 2.11. It can be seen that by changing the internal reflectivity on the back side from $65 \%$ to $95 \%$, in this model a gain of $1.1 \mathrm{~mA} / \mathrm{cm}^{2}$ can be achieved. Obviously, making an effort to implement elaborate light trapping textures in a solar cell and thus creating long internal light paths in particular makes sense in combination with taking care about excellent internal reflection properties as can be seen in Figure 2.11. Additionally, a back side passivation layer will play a major role when it comes to the 
realisation of the diffractive grating on the back side as to be introduced in section 5.2 .

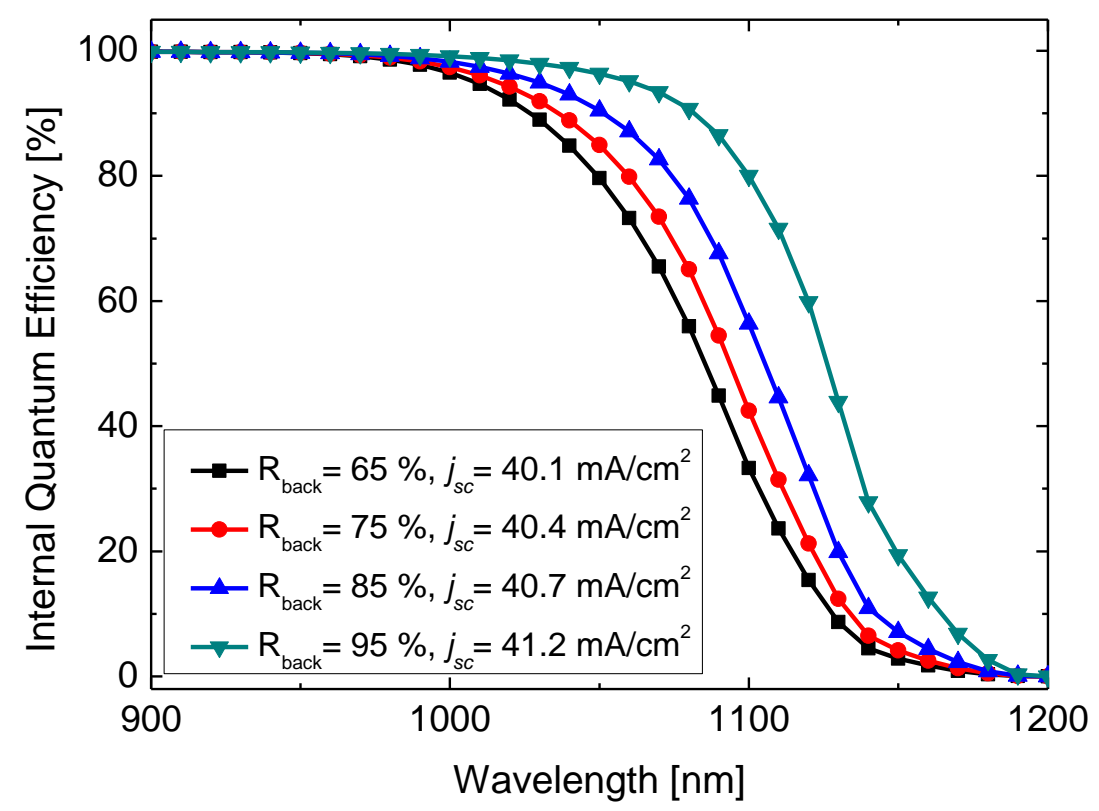

Figure 2.11: Calculated internal quantum efficiencies for a pyramidal textured silicon solar cell using PC1D to visualise the influence of the internal reflectivity at the solar cell back side on light trapping properties. Additionally, the resulting short-circuit current densities are shown in the legend (for a random pyramid texture with a single layer SiN ARC; the front side reflectivity was calculated using the OPAL tool [48]). Relevant solar cell features are: $200 \mu \mathrm{m}$ thickness, $1 \Omega \mathrm{cm}$ p-type FZ material, Gaussian $80 \Omega / \square$ emitter. Surface recombination velocity is omitted and internal reflection properties for the front side are oriented to the ones published in [45, 47].

A concept related to the introduction of passivated back surfaces for the PERC concept is the local contacting of a solar cell exhibiting this feature. A very promising and already very well understood means of achieving this is via laser-fired-contacts (LFC) [49]. In the LFC process an aluminium layer is deposited on the back side of the dielectric passivation layer (e.g. silicon oxide or silicon nitride) by screen printing or evaporation processes. Then, a pulsed laser is used to locally fire the aluminium through the dielectric layer and form an alloy with the silicon bulk. Thus even a beneficial local $p^{+}$region can be realised beneath the metal contact, and, since only a small fraction of the substrate's back surface is in contact with the metal, very low surface recombination velocities can be obtained.

Regular textures, e.g. inverted pyramids or honeycomb textures, are applied in silicon solar cells for reaching the highest efficiencies on the laboratory scale both for monocrystalline and for multicrystalline silicon (respectively [5, 31]). Especially for multicrystalline silicon the optical behaviour of a standard isotextured solar cell can strongly be improved. Therefore, there is on-going research for developing processes 
to realise defined regular textures feasible for industrial fabrication. This issue is tackled by the development described in this work and the state-of-the-art is therefore explained in section 3.2.

Besides standard micron sized textures on the front surface (also the case for the honeycomb texturing), there has been research about introducing diffractive features in solar cells to enhance light trapping [50]. In the following chapter, an overview of optical mechanisms to enhance absorption via surface texturing will be given. Furthermore, electrical effects as a result of the structuring of semiconductor surfaces have to be considered. Regarding these aspects, the development of an up-scalable process chain to realise sophisticated surface textures is described within this work for micro- and nano-scaled texturing concepts.

Figure 2.12 shows one possible adapted process flow incorporating a defined texture on the front side in a PERC cell that uses some of the measures described in this section. Also shown is a sketch of the resulting cell architecture.
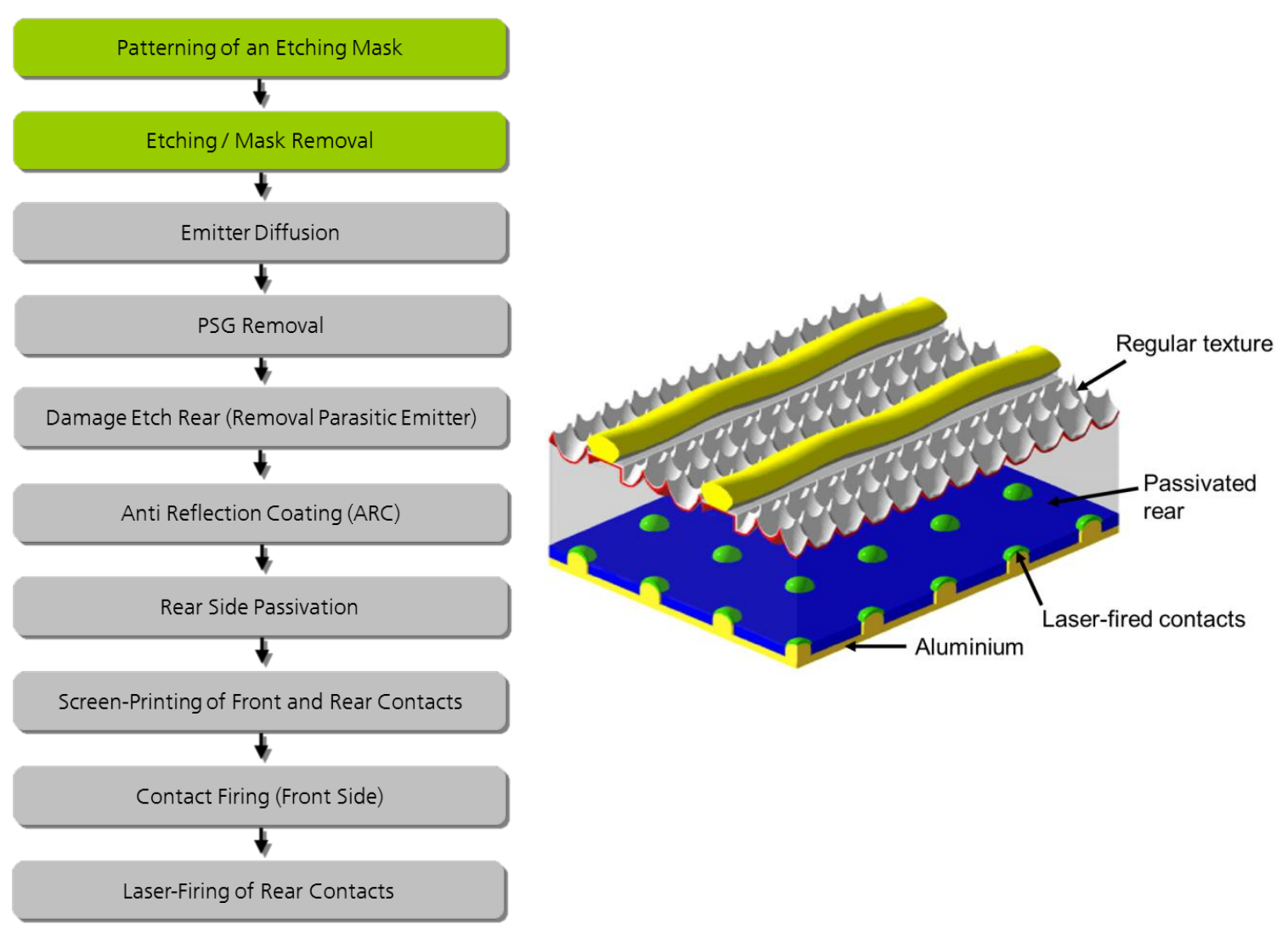

Figure 2.12: Left: Possible process flow of a PERC cell with a defined texture [51]. The process steps related to the defined pattern transfer as developed within this work are marked green. Right: The resulting cell structure is visualised schematically. Graphic modified from [21]. 


\section{Textured silicon surfaces - optical \& electrical effects}

In 1960 it was shown that by texturing the front of a silicon solar cell, its efficiency can be increased [52]. Within this chapter, basic mechanisms leading to this efficiency enhancement shall be explained. In this context, the optical behaviour of textured surfaces and the resulting benefits for solar cells will be described, followed by a review of the evolution of investigated texture types for silicon solar cells. However, there are not only positive aspects related to a surface texturing. Increasing the solar cell surface is related to increased recombination of free charge carriers. Furthermore, processes used for the texturisation can also lead to a deterioration of electrical properties. These negative aspects shall be the topic of the second part of this chapter.

\subsection{Optics for solar cells}

\subsubsection{Two effects: Reduction of reflectivity and light trapping}

The efficiency of power conversion in solar cells relies in the very first place on the absorption of impinging electromagnetic radiation in a semiconductor material. All radiation that is not absorbed within the semiconductor but reflected, transmitted or absorbed within other materials (e.g. metallisation) cannot be converted into extractable electrical power. Thus, one important field for optimising silicon solar cells is improving their optical characteristics.

In fact, for better understanding, the optical improvement can be tackled by two measures:

(i) Reflection at the front interface has to be minimised.

(ii) Internal light paths have to be increased as much as possible.

The first measure is generally important for solar cells, as any reflection at this interface is lost ${ }^{4}$. The second measure is important because of the spectral absorption behaviour of the indirect semiconductor silicon as shown in Figure 2.4. Radiation with photon energies close to $E_{\text {Gap }}$ are absorbed only very weakly and thus require very long internal path lengths. This in particular will gain in

\footnotetext{
${ }^{4}$ This statement is not entirely true when a solar cell is encapsulated in a module. There, internal reflection in the module glass can lead to a re-use of these photons.
} 
importance for decreasing cell thicknesses, a trend being followed to reduce costs in industrial fabrication.

The description of the interaction of light with textured or coated surfaces can follow different approaches. Effects relying on interference require a treatment of light as electromagnetic waves. This will be considered in the following for optical effects in thin layers. The effects caused by textured surfaces strongly depend on the spatial frequency of features. While small features with structure dimensions in the range of optical wavelengths and below require wave optical considerations to capture effects like e.g. reflection, transmission or propagation directions, larger features can be described using ray optical approaches. In the latter, no wavelength-dependent effects are considered except for dispersion. However, in modern ray tracing software, interference effects in thin layers can be considered, which are typically modelled using the transfer-matrix formalism [53]. When calculating photo-current densities $j_{p h}$ the quantized nature of light is applied and photons are counted per unit area and time.

Silicon solar cells convert radiation of a broad spectral range into electrical energy. Measures to improve the optical characteristics of solar cells can be strongly wavelength selective. Therefore, it is important to define a figure of merit that allows the comparison of results. This can be achieved by weighting spectral reflection measurements $R(\lambda)$ with the photon flux of the solar spectrum $\Phi(\lambda)$ (for non-concentrating systems, the AM1.5g is the relevant spectrum) via

$$
R_{w}=\frac{\int_{\lambda_{1}}^{\lambda_{2}} R(\lambda) \cdot \Phi(\lambda) d \lambda}{\int_{\lambda_{1}}^{\lambda_{2}} \Phi(\lambda) d \lambda}
$$

with integration boundaries $\lambda_{1}$ and $\lambda_{2}$ describing the spectral range useable for silicon solar cells. For determining $R_{w}$, please note that $\Phi(\lambda)$ is the photon, and not the solar spectrum in Watts per square meter, how it is often visualized. Figure 3.1 shows this solar photon flux per unit wavelength per square meter per second. The maximum of the AM1.5g photon flux can be found at a wavelength of around $650 \mathrm{~nm}$. Integrating this photon flux for energies above $1.12 \mathrm{eV}$ and multiplying it with the elementary charge $q$ leads to a maximum of the achievable short-circuit current density $j_{s c}$ of $43.2 \mathrm{~mA} / \mathrm{cm}^{2}$. 


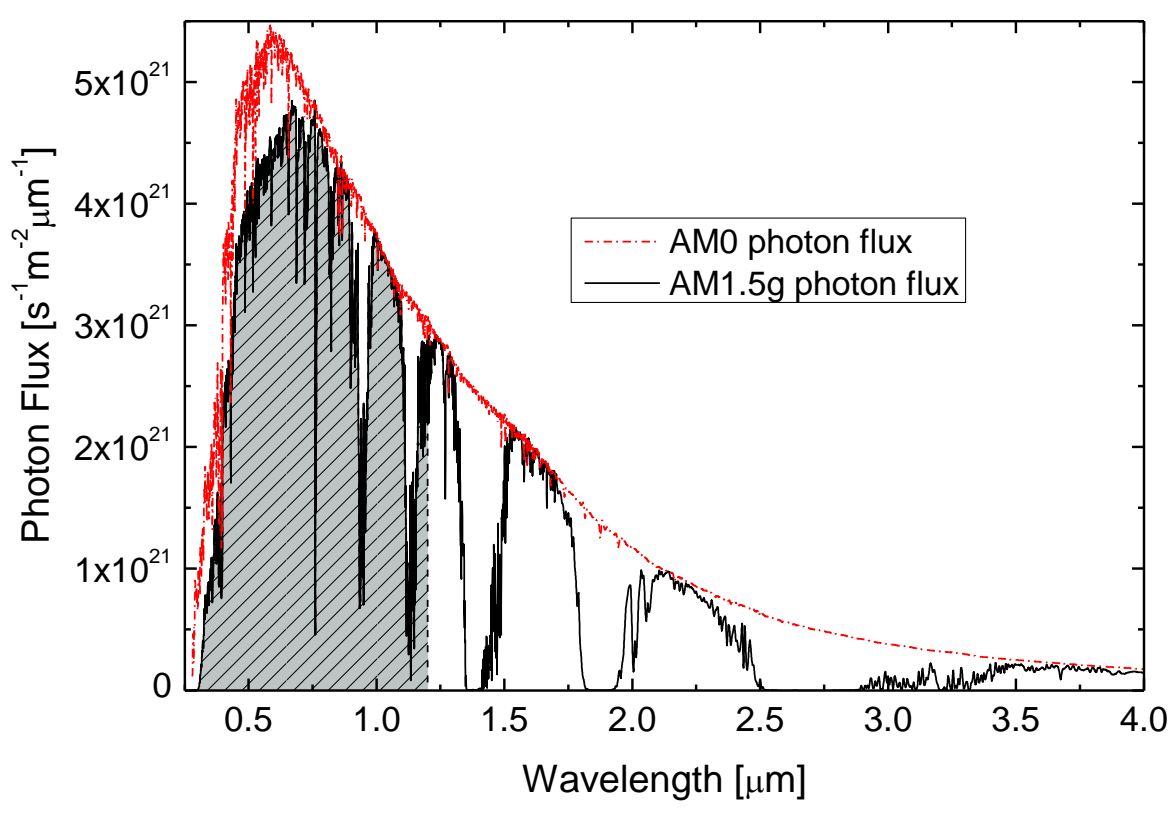

Figure 3.1: Photon flux of the solar spectrum. AM stands for air mass and describes the path length through the earth's atmosphere. Thus AMO denotes the flux before entering the atmosphere. AM1.5g is the global photon flux (containing both direct and diffuse radiation) travelling through 1.5 times the air mass. This value is typically used to characterise solar cells (that are not based on optical concentration). The grey and hatched area indicates the spectral range relevant for silicon solar cells.

\section{Interference in thin layers}

A very well-known effect to reduce front surface reflection is based on the use of interference effects in thin layers. A schematic view of such a system is shown in Figure 3.2. The reflection reduction of this single layer antireflection coating (ARC) is based on interference of the reflected waves at the interfaces between the incident medium and the ARC and between the ARC and the substrate material. By making use of destructive interference, the reflection can be oppressed completely. To this end, two prerequisites have to be fulfilled. First, the amplitudes of the reflected waves have to be equal. This is related to the refractive indices of all three involved media and can be calculated using Fresnel's equations. Second, the phase difference between the reflected waves has to be $\frac{\lambda}{4}$. Therefore, the optical thickness of the ARC has to be adapted to ensure this effect (therefrom the name $\frac{\lambda}{4}$-layer can be deduced). This further implies that, when using a single layer $A R C$, reflections can only be suppressed totally for distinct wavelengths. These two prerequisites of amplitude and phase conditions can be used to derive the following two equations to determine ideal refractive index $n_{A R C}$ and layer thickness $d$ to totally supress reflection for given incident and substrate materials 
with refractive indices $n_{1}$ and $n_{s}$, a design wavelength $\lambda_{0}$ (in vacuum) and normal incidence:

$$
n_{A R C}=\sqrt{n_{1} \cdot n_{s}} \quad \text { and } \quad d=\frac{\lambda_{0}}{4 \cdot n_{A R C}}
$$

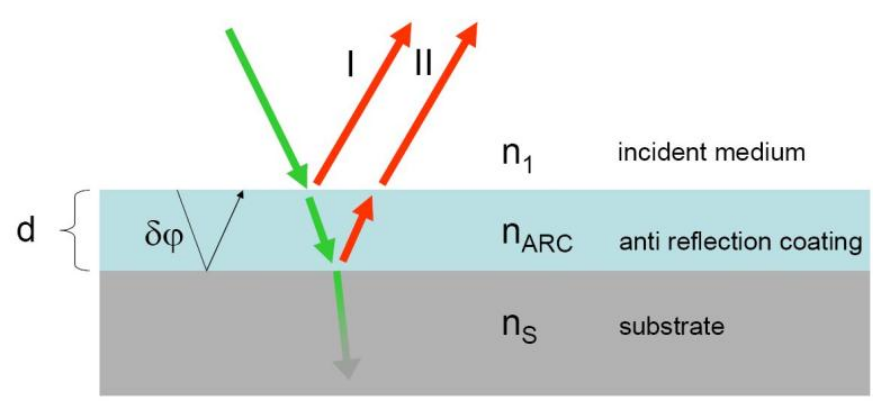

Figure 3.2: Sketch of a single layer antireflection coating. Graphic taken from [54].

For silicon these considerations lead to an optimum $n_{A R C}$ of around 2 or 2.3 for air or glass as the incident medium respectively. Corresponding ideal layer thicknesses are in the range of 80 or $70 \mathrm{~nm}$ to optimise the performance of the ARC at the design wavelength of $650 \mathrm{~nm}$. The application of an ARC to solar cells is standard in solar cell fabrication. The dominating material for this purpose is silicon nitride, which can be applied via sputtering or PECVD. The realisation of homogeneous layers improves the front surface reflection properties but does not induce any internal path length enhancements.

\section{Textured surfaces}

Another approach that in principle is capable of contributing to both measures of reflection reduction and path length enhancement is introducing a texture into the solar cell surface. However, depending on the type, position (front or rear side of the wafer) and dimensions of a surface texture, not necessarily both effects can be exploited. Depending on feature sizes, optical effects of textured surfaces can be explained by geometric optics or require more sophisticated wave-optical calculations to fully describe occurring effects.

Figure 3.3 visualises potential optical effects caused by textured surfaces. These exemplary cases of three ratios of wavelength $\lambda$ to pattern period $\Lambda$ are illustrated for periodic features. However, in principle conclusions drawn from this special case of periodic features can be extended to non-periodic surface structures. 


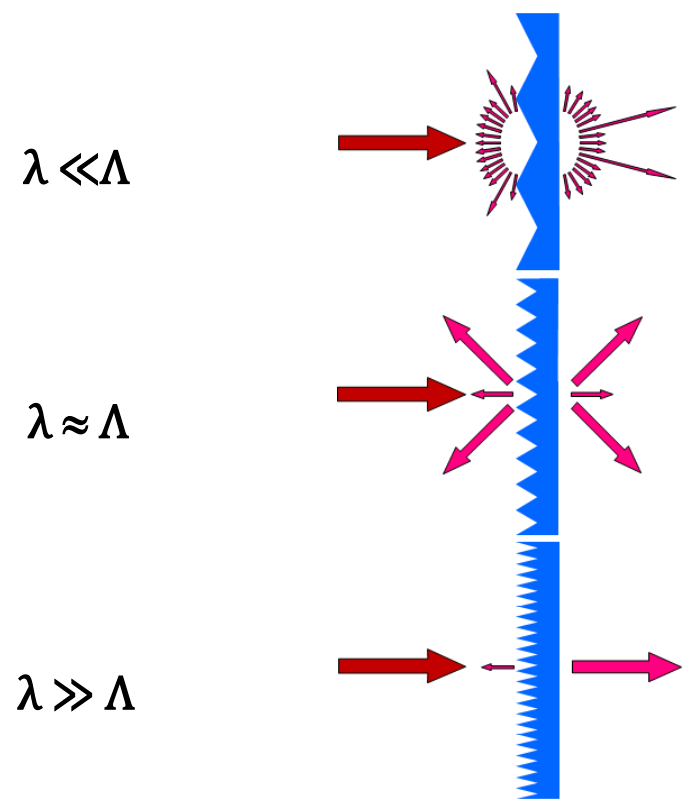

Figure 3.3: Schematic view of optical effects caused by periodically textured surfaces of varying special frequency for a given wavelength. Graphic taken from [55].

\section{$\lambda \ll \Lambda$}

For $\lambda \ll \Lambda$ a large number of diffraction orders can propagate. The intensity distribution of these diffraction orders strongly depends on the shape of the surface pattern. For a very high ratio of $\Lambda$ to $\lambda$, this effect leads to the geometric optical limit of refraction as can be described by Snell's law. Because of these effects related to a change of propagation direction, an internal path length enhancement in solar cells can be achieved. A second effect, when treating the effects occurring in these large structures geometric optically, is that multiple reflections can occur as shown in Figure 3.4. Here, a V-groove texture serves as an example. This effect additionally reduces the overall reflection at the front surface. The standard simulation method for patterned surfaces of present dimensions is ray tracing. Stochastically or randomly textured surfaces can be treated similarly, and may typically be sufficiently described by ray optics if feature sizes are large enough compared to wavelengths of the interacting light. 


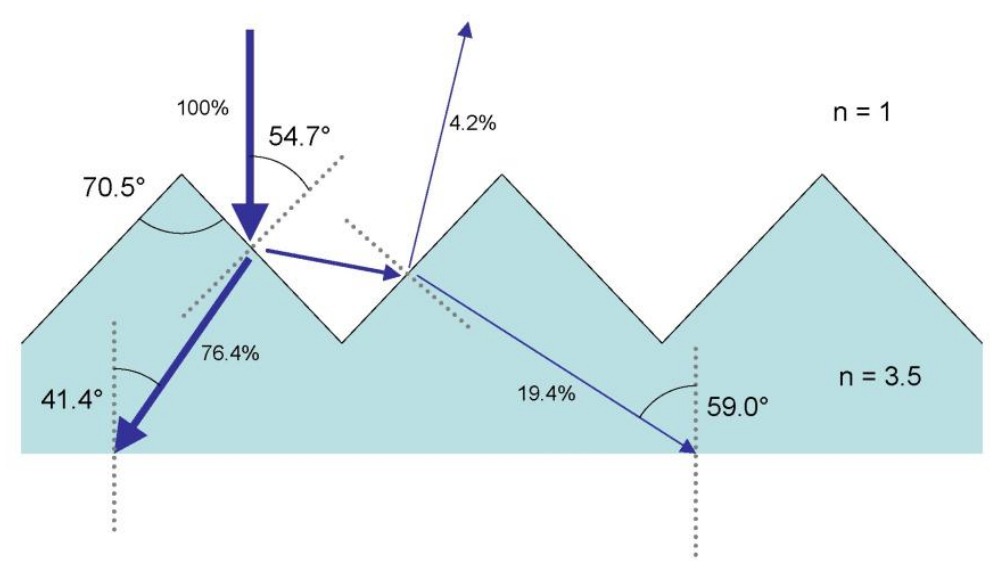

Figure 3.4: Schematic diagram of light paths in a V-groove pattern for the case of a double reflection on opposite surfaces. Angles correspond to $<111>$ planes in a $<100>$ silicon wafer. The figures given represent the case for light with a wavelength of $1040 \mathrm{~nm}$ and a solar cell with an antireflection coating of silicon oxide with a thickness of $105 \mathrm{~nm}$. Graphic taken from [54].

\section{$\lambda \approx \Lambda$}

For the interaction of pattern sizes and wavelengths of similar dimensions, interference effects leading to distinct diffraction orders dominate the optical behaviour. Because of these effects, the behaviour of the textured surface is strongly wavelength dependent in terms of reflection and transmission properties. Well defined gratings can be used to achieve both reflection reduction as well as a strong path length enhancement. However, this will typically be possible only for a narrow spectral range. The simulation of these features requires a rigorous solution of Maxwell's equations. Stochastic features in these dimensions can be described using scattering theories. Under the assumption of spherical features, the formalism of Mie scattering can be applied to describe complex scattering distributions. In this theory, each point on the surface of the spherical particle acts as source of spherical waves. The superposition of these waves leads to the scattering distribution.

\section{$\lambda \gg \Lambda$}

Periodically patterned surfaces with features the size of well below the incoming wavelengths can be described using effective medium theories. This means the structures are not resolved by the light and therefore act as a medium with an effective refractive index. As a consequence, these small features have influence on the amount of transmission and reflection but do not induce any light guidance since no change of propagation direction occurs. A special case of these subwavelength structures are the so-called moth-eye structures, in which a conical 
feature shape leads to a graded transition of effective refractive indices. Thereby, a very efficient and broadband antireflection effect can be achieved. This effect was discovered by Bernhard [56]; first artificially originated moth-eye surface patterns were reported by Clapham and Hutley [57] and a comprehensive study on this topic can be found in [58]. Looking at stochastic structures with very small structure dimensions, scattering effects also occur. These, in principle can be described by Mie or Rayleigh scattering (depending not only on mean feature sizes, but also on mean spatial frequency of the surface structures). Additionally, the approximation of regarding stochastically arranged small surface structures as a superposition of several high frequency periodic gratings can be applied. It can be concluded that reflection can be suppressed very well, but scattering efficiencies, in particular into large angles, are not likely to be very high for stochastic features with mean feature sizes far smaller than $\lambda$.

\section{Light trapping}

The expressions light trapping or also optical confinement are often used in the context of making light that enters the solar cell bulk travel long paths within the semiconductor. To this end, both a change of propagation direction into oblique angles as well as high internal reflection properties are the key to achieve pronounced light trapping effects. For silicon, the effectiveness of light trapping schemes is assessed by the absorption behaviour in the weakly absorbed wavelength regime close to $E_{G a p}$. Strategies to change propagation directions can be based on randomisation by applying e.g. Lambertian scattering schemes, geometrical effects aroused by surface structures that cause refraction or diffractive effects introduced by gratings. An important parameter describing internal reflection properties is related to the escape cone. This escape cone is defined by the critical angle $\theta_{\text {crit }}$, below which no total internal reflection occurs. This critical angle is solely defined by the refractive index $n$ of the semiconductor by

$$
\theta_{\text {crit }}=\operatorname{ArcSin}\left(\frac{1}{n}\right)
$$

There are theoretical works trying to quantify maximum path length enhancements for these different schemes of manipulating propagation directions. Götzberger analysed in 1981 the maximum absorption enhancement just by regarding the escape cone in silicon and assuming an ideal diffuse or Lambertian reflector as shown in Figure 3.5. There, he finds for a relatively simple model that only $8.5 \%$ of the radiation reflected on the rear side is re-emitted [59]. 


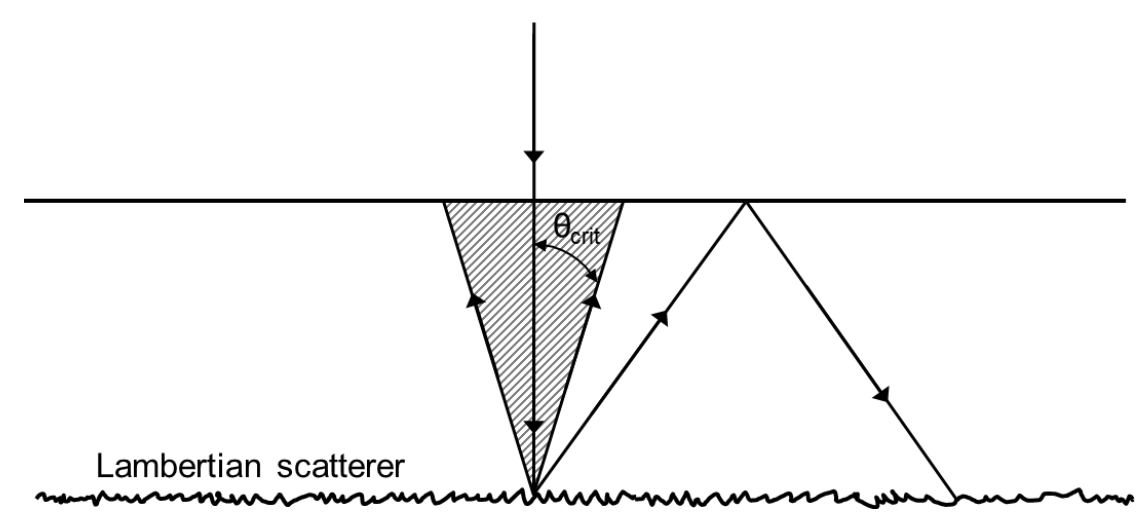

Figure 3.5: Sketch of a solar cell comprising a flat front and a diffuse rear side reflector. The escape cone with the critical angle $\theta_{\text {crit }}$ is drawn, within which light can escape the solar cell. Redrawn from [59].

The most renowned work, going in a similar direction, is probably that of Yablonovitch [60]. This work leads to the conclusion that a maximum path length enhancement, based on isotropic scattering and thus a total randomisation of light paths within the cell, equals a factor of $4 n^{2}$. This result, well-known under the expression Yablonovitch limit, was confirmed by similar theoretical considerations in [11] as well as numerical simulations in [61]. Thus, in silicon, a maximum path length enhancement of a factor of around 50 can be achieved compared to a planar wafer, applying randomisation schemes.

The work of Miñano [62] describes a possible way to overcome this Yablonovitch limit. There, it is shown that internal path lengths can be enhanced drastically by the application of angular selective filters. The solar radiation enters the solar cell only under a very small solid angle. Restricting the transmission of radiation at the front surface to this solid angle and applying a randomisation scheme on the rear, the factor of a potentially achievable path length enhancement can be as high as 46200. However, this would require an absolutely perfect tracking of the solar cells.

In [11] the idea is formulated that isotropic randomisation as fulfilled by Lambertian scattering might be ideal for devices with light impinging from arbitrary directions. However, when dealing with distinct and predictable angular distributed irradiance, there might be solutions, where path length enhancement schemes other than isotropic scattering are superior and thus also the Yablonovitch limit can again be exceeded.

Thus, surface textures that induce a path length enhancement not by randomisation but by refractive or diffractive effects are, in principle, capable of beating the Yablonovitch limit. The advantage of making use of refractive effects is that there is no wavelength dependency besides colour dispersion. However, for 
the sake of finding an upper bound of maximum achievable path length enhancements, gratings are more suited. There, as an ideal model it is possible to think of having solely one distinct diffraction order holding all energy (no intensity in the zero order). Peters et al. formulated a maximum path length enhancement introduced by an ideal grating on the rear side of a silicon solar cell published in [54]. This calculation is mainly based on the thought of sunlight having a certain divergence and that the interaction of light with different media and any optical elements (as also the ideal grating) has to obey the conservation of étendue ${ }^{5}$. A sketch of the considered setup is shown in Figure 3.6. As a result, a maximum path length enhancement factor of $853 n$ (2980 for a refractive index of 3.5) can be achieved.

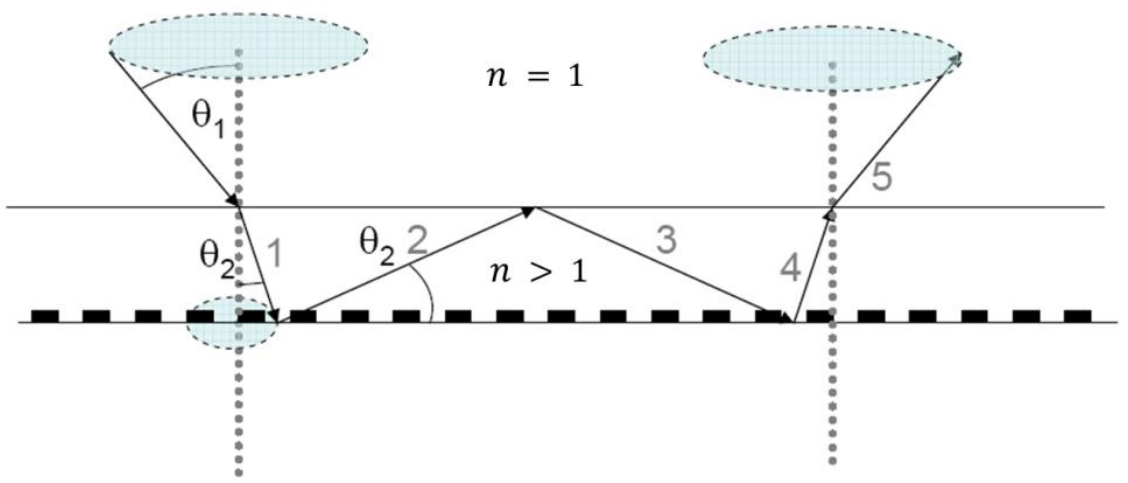

Figure 3.6: Schematic diagram of the paths of the light in a solar cell with a rear side grating. The blue ellipses indicate the divergence of sunlight. Graphic taken from [54].

The result of Peters et al. is based on an ideal model for one single wavelength. More recent publications of Mellor et al. [63] and Peters et al. [64] show that aiming for having only the plus/minus first diffraction orders, which shall travel nearly parallel to the wafer surface, is not the optimal case. In both publications, numerical studies on the absorption enhancement in around $40 \mu \mathrm{m}$ thick solar cells were performed considering the entire solar spectrum. The results of both authors are in good agreement and show that optimum periods of rear side gratings are in the range of $1 \mu \mathrm{m}$. Mellor additionally showed that for thicker wafers the period should be slightly increased. This indicates that when dealing

\footnotetext{
${ }^{5}$ The étendue is related to the angular distribution as well as the surface element crossed by a spatially limited wave packet. The expression ètendue can be seen thermodynamically as optical analogy to the entropy. When a spatially limited wave packet is interacting with ideal surfaces, resulting propagation for e.g. reflection or refraction induces no change in the étendue. However, any scattering or diffuse reflection is related to an increase in the étendue.
} 
with more than one wavelength, gratings perform better when more diffraction orders are permitted. In this case, it can be concluded that plus/minus first diffraction orders are just outside the escape cone and thus all diffraction orders are totally internally reflected for a flat front surface.

\subsubsection{Evolution of surface texturing for silicon solar cells}

\section{Overview of etching technologies (maskless)}

As mentioned in the beginning of this chapter, silicon solar cells were textured to improve their absorption behaviour as early as 1960 [52]. At that time, ultrasonic cutting was applied to fabricate tetrahedral inverted pyramids. Another approach in those early days of research on photovoltaics was based on sandblasting of silicon surfaces as patented by John in 1969 [65].

At the same time, it was found that alkaline etching processes in silicon lead to anisotropic etching behaviour of crystal planes [38]. As described in chapter 2, the silicon crystal shows the highest density of atoms in the $<111>$ planes, which, as a result of the stronger binding energy in this plane, are etched slowly compared to other (in particular the $<100>$ ) planes. Therefore, these anisotropic etching processes on $<100>$ orientated wafers lead to surfaces comprising of intersections of $<111>$ planes, which are consequently pyramidal patterns. Since then, these pyramidal textures evolved into the standard texture for monocrystalline silicon wafers. Previously, this alkaline texturing process was also applied for multicrystalline silicon; however, the non-unique crystal orientation in this type of material leads to a very inhomogeneous optical quality. Nonetheless, this anisotropic texturing process affords some benefit for multicrystalline silicon and the aqueous alkaline solutions are easily handed. Therefore, this etching process was standard for both of these types of materials for a relatively long time. Typically used etchants for this application were, and still are, potassium or sodium hydroxide ( $\mathrm{KOH}$ or $\mathrm{NaOH}$, respectively). The first commercially available pyramidally textured monocrystalline solar cells were fabricated for space applications in 1975 [66]. Figure 3.7 exemplarily shows SEM images of textures on mono- and multicrystalline silicon that result from alkaline treatment. 

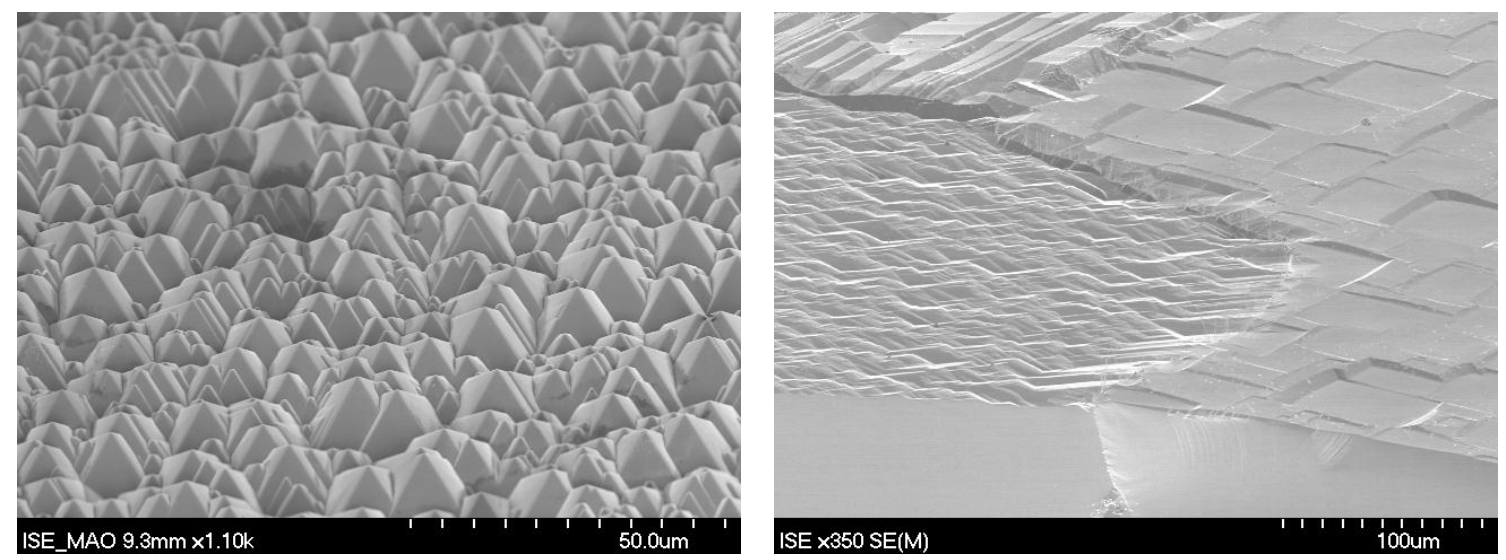

Figure 3.7: SEM micrographs of textured silicon surfaces realised via alkaline etching. On the left hand side a pyramidal texture as obtained on $<100>$ orientated monocrystalline silicon is shown. On the right hand side, the texture variation between differently orientated grains on multicrystalline silicon is visible.

The inhomogeneity of alkaline textured multicrystalline silicon was tolerated for a long time, despite a wet-chemical etching technique that works independently of crystal orientations being published in 1959 [67]. There, the etching processes of silicon using aqueous solutions of hydrofluoric and nitric acid ( $\mathrm{HF}$ and $\mathrm{HNO}_{3}$ respectively) are described. The etching mechanism is based on an oxidation of silicon by $\mathrm{HNO}_{3}$ and a removal of this oxide layer by HF. The introduction of this so-called acidic isotropic texture (often called an isotexture) into industrial environments followed in the 2000's [68]. The handling of these acidic texturing processes is demanding, since the involved reactions are strongly exothermic.

There are ways to obtain surface patterns of roughly homogeneous and predictable spatial frequencies for these maskless wet-chemical etching processes. Both for alkaline texturing of monocrystalline as well as acidic texturing of mutlicrystalline silicon, so-called bubble-masking effects can be applied by adding agents to the etching solution $[69,70]$. Especially for the isotexture, the etching process has to start at nucleation points so that no planar surface results (as a result of a homogeneous etching). A simplified process for the effective texturing of multicrystalline silicon using acidic etching processes is based on using saw damages on wafers as seeding or nucleation, in this way a homogeneously distributed texture can be achieved $[40,71]$. Figure 3.8 shows two photographs of multicrystalline silicon wafers, one textured using an alkaline solution, the other using an acidic solution. The effect of obtaining a texture independently of the crystal orientations of different grains by acidic in contrast to the alkaline texturing can clearly be seen. 

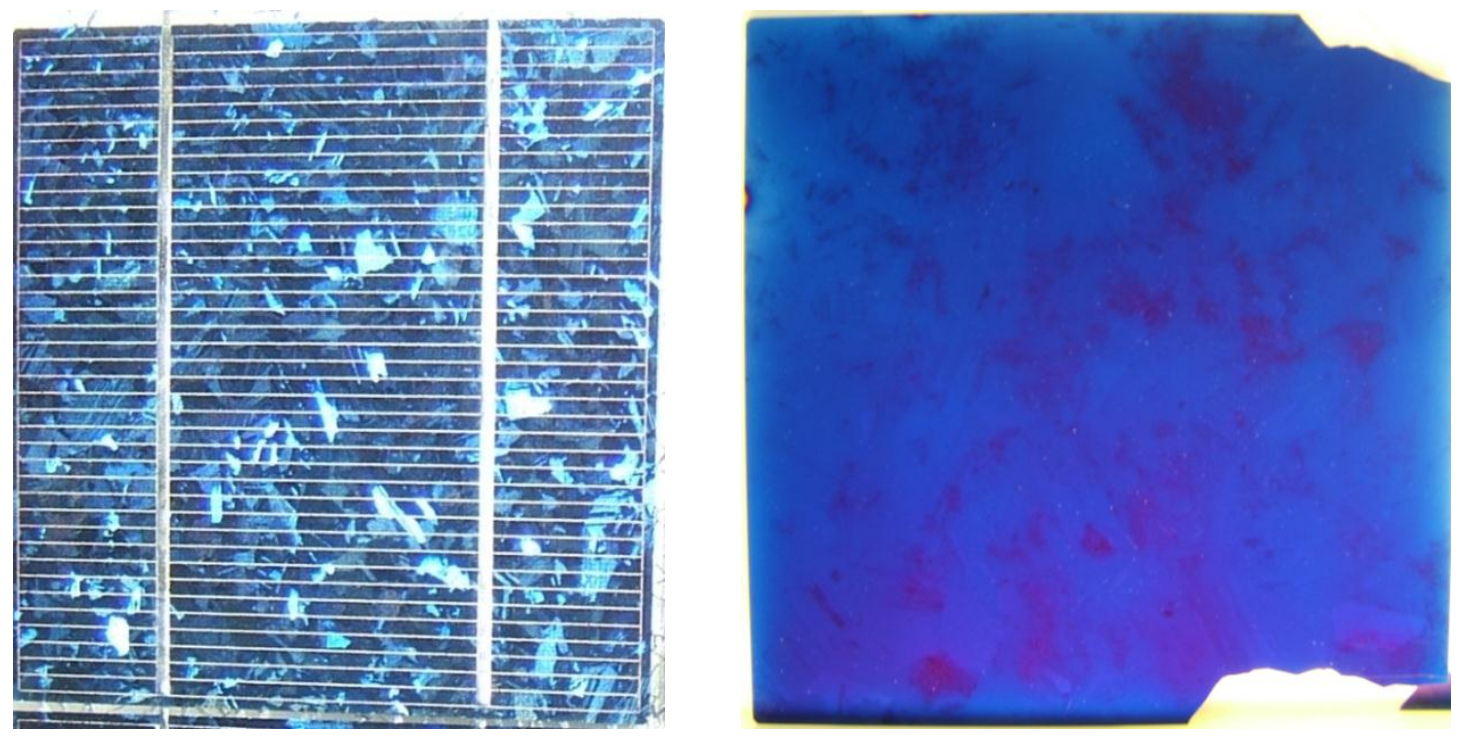

Figure 3.8: Photographs of two multicrystalline silicon wafers with silicon nitride ARC. On the left hand side a wafer textured by alkaline etching is shown. This wafer has contact fingers and busbars. On the right hand side the homogeneous texture of an isotropic texturing process using an acidic texturing solution can be seen.

At the moment, alkaline and acidic wet-chemical texturing are the state-of-the-art texturing processes for mono- and multicrystalline wafer based silicon solar cells respectively. These processes are applied without any etching masks leading to socalled random pyramids and the isotexture. Reflection measurements of silicon substrates with these surface textures and no antireflection coating are shown in Figure 3.9. There, it can be seen that the pyramidal texture is by far superior to the isotexture in terms of optical quality. This is related to the isotropic etching behaviour of the acidic texturing leading to spherical profile shapes. In the long wavelength range, comparison of these measurements is difficult, since the increase depends on the wafer thickness and also on the rear surface topography. In case of the isotropic texture, the rear side is also textured and the substrate was slightly thinner.

Feature sizes of these two types of textures are in the range of several microns and therefore can be simulated using geometric optics via ray tracing. There are also publications describing the optical behaviour both of pyramidal as well as isotextured surfaces using analytical approaches combined with geometrical considerations and, in case of the isotexture approximations of mean texture profiles. The analytical model for pyramidal textures by Mclntosh and co-workers is based on the evaluation of possible distinct light paths for different types of pyramidal textures $[48,72]$. This model is implemented in the freeware tool Opal. Obtained results for values of the weighted reflectance are in good agreement 
with the measured data found both within this work and in the literature. Weighted reflectance values of pyramidally textured silicon wafers without ARC can be expected to vary between 11 and $13 \%$, depending on the fraction of planar areas in between pyramids, the wafer thickness and the rear surface quality.

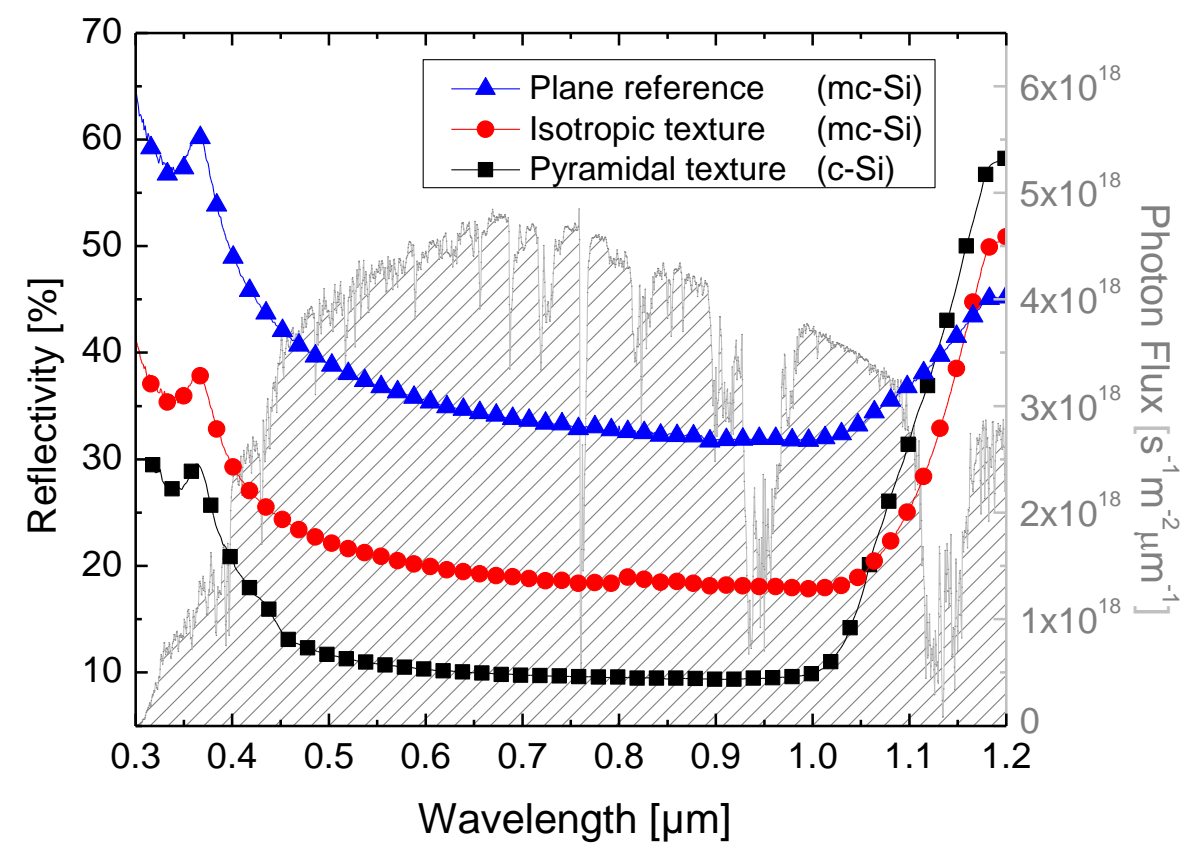

Figure 3.9: Reflection measurements of silicon wafers with different surface topography. In the background the AM1.5g photon flux is visualised.

\section{Alternative techniques for maskless texturing processes}

Another kind of surface texture is known under the expression porous silicon. This porous silicon layer can either be formed by chemical etching, using a specific aqueous solution of $\mathrm{HF}$ and $\mathrm{HNO}_{3}$, or by electrochemical etching using $\mathrm{HF}$ [73]. The main effect caused by this porous silicon texture mentioned in the literature is related to treating this porous layer as an effective medium. The porous layer formed by electrochemical etching consists of interconnected nanocrystalline features with sizes around $1-10 \mathrm{~nm}$ [74]. Thereby, it can be explained that this layer more or less acts as an antireflection coating with an effective refractive index [75]. For these very small feature sizes no significant scattering or diffraction for relevant wavelengths can be expected. In terms of reduction of the front surface reflectivity, very good results were achieved using these porous silicon antireflection coatings; however, for published data on cell results, this high optical quality could not be transferred onto electrical quality at the cell level. These problems will be described in the next section, which discusses the electrical effects of textured surfaces. 
Maskless plasma etching processes were also extensively investigated for solar cell texturisation. Especially the combination of reactive ion etching and self-masking effects (further explanation in section 4.4) are interesting since this combination allows the realisation of needle like textures. This texture leads to very effective antireflection properties and is therefore often mentioned in literature under the expression black silicon [76, 77]. The optical effect leading to this suppression of reflection is, depending on feature sizes, most often related to sub-wavelength features, which form an effective graded refractive index transition (analogue to the moth-eye effect described before). Here, excellent values for reflection can be found throughout the literature; however, similarly to the porous silicon layers, solar cell results did not match this high quality, again due to a degradation of electrical properties, as shall be reported in section 3.3.2. In contrast to the porous silicon concept, the maskless plasma texture is applied by at least one cell manufacturer, namely Kyocera. Figure 3.10 shows exemplarily an SEM micrograph of a black silicon texture.

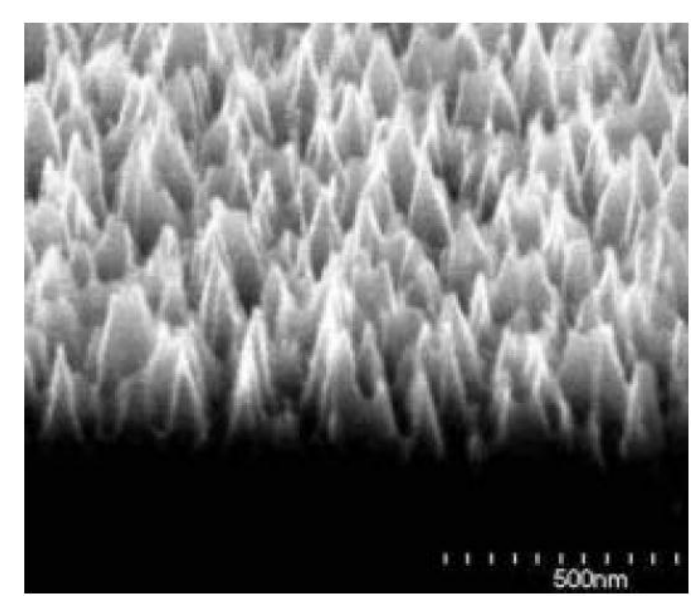

Figure 3.10: SEM micrograph of a black silicon textured wafer. Graphic taken from [78].

\subsection{Defined texturing processes}

To optimise the optical properties of solar cell textures a wide range of defined texturing processes as well as different types of tailored textures were investigated. The processes can mainly be divided into the two groups: direct patterning and two-step processes. In the latter, an etching mask is initially patterned, which afterwards is applied to achieve a defined pattern transfer into the silicon. In the beginning of this section, general approaches and technologies will be described. Afterwards, two concepts followed in this work will be explained in more detail.

The first direct patterning approaches to obtain pre-defined texture geometries were based on mechanical material removal such as ultra-sonic cutting, as 
mentioned in the very beginning of this chapter [52]. Sawing processes were afterwards investigated, mainly to fabricate $V$-groove but also pyramidal shaped geometries $[79,80]$. An interesting aspect for the sawing of $V$-groove textures is that the geometry is not restricted to crystallographic orientations in contrast to when etching with $\mathrm{KOH}$. Damages introduced by the sawing process typically have to be removed by wet-chemical etching.

Another direct patterning approach is based on direct laser ablation of silicon [81]. This technique was mainly investigated for improving texture quality on mc-Si and is typically combined with subsequent wet-chemical, but also plasma, etching [82]. This additional etching process is necessary to remove laser induced damages.

The highest number of degrees of freedom is given when applying texturing processes, which rely on the initial patterning of etching masks. Figure 3.11 visualises the very principle of this two-step texturing process. Furthermore, an overview of possible technologies/materials for these steps is given.

\section{Masking:}

Organic or inorganic resist materials applied from liquid phase (spin-, spray-, dip-coating...); inorganic layers applied via vacuum deposition.

\section{Etch mask patterning:}

Photolithography, hot embossing / nanoimprint lithography, micro-contact printing, inkjet, laser ablation etc.

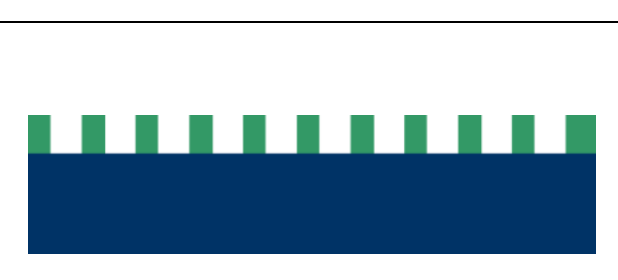

\section{Etching process:}

Wet or plasma etching.

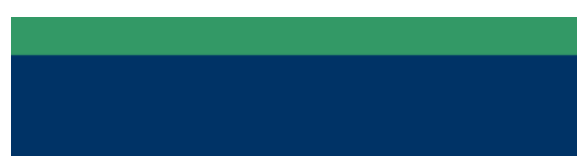

Figure 3.11: Schematic view of a defined texturing process based on the use of etching masks. An incomplete selection of possible materials or processes for each step is listed.

Classically, this basic principle of defined texturing is realised using photolithography processes as done in the microelectronics or micro-electromechanical-systems (MEMS) industry [83]. Extremely well defined conditions have to be ensured and thus most often a photoresist is patterned, which is then used to pattern a hard mask (e.g. silicon oxide) between the photoresist and the silicon 
substrate. The hard mask is patterned e.g. in an HF-dip and can then be applied as an etching mask in a wide range of etching processes. The advantage of using a hard mask compared to the direct use of polymeric photoresist as an etching mask is the potentially far higher etching selectivity. This selectivity describes the ratio of etching rates of the material to be removed to that of the masking material. Photolithography is the standard process when it comes to high definition pattering in the semiconductor industry, MEMS as well as laboratory scale processes in photovoltaics. Feature sizes for full-wafer mask aligner ${ }^{6}$ processes can get down to around $1-2 \mu \mathrm{m}$. However, the requirements set on processing conditions and substrate pre-treatment are by far too challenging to transfer this technology to industrial scale solar cell fabrication. Issues hindering this process from being applied in these environments are mainly cost related (e.g. expensive resist materials, additional process steps, processing times), but are also affected by established (pre-) processes such as the wire-sawing, which causes potentially very rough surfaces even after wet-chemical saw damage removal (especially for multicrystalline silicon). These rough surfaces can be removed by polishing processes; however, this further increases processing complexity and thus costs. Photolithography can be performed on these rough surfaces, but a reduced resolution has to be accepted.

Therefore, there is considerable research activity aimed at finding a substitution for photolithography to allow the realisation of defined high-resolution features in industrial scale processes. Alternative approaches for the patterning of etching masks that can be found in the literature are laser ablation or inkjet printing. Laser ablation can be used to pattern hard etching masks (e.g. silicon nitride) to allow acidic etching processes for mc-Si [84]. Disadvantages of laser ablation processes are a limited resolution, typically greater than $10 \mu \mathrm{m}$, and long processing times. The serial ablation of single etching pits using state-of-the-art pulsed laser systems is very time consuming. Therefore, diffractive optical elements (DOE) for the multiplication of a laser beam can be applied to increase processing speed [85].

The inkjet patterning of etching masks can be conducted using different media. There is a wide range from standard polymeric resist materials to hotmelt inks, which can be used as an etching mask [86]. In [87], a so-called direct patterned etching approach is described, where first an acidic water-soluble polymer layer is applied on the whole surface of a dielectric hard mask layer. In a second step, an inkjet process is used to locally form the acidic etching solution by printing an aqueous solution of fluoride ions. Afterwards, the wafer is washed in de-ionised

\footnotetext{
${ }^{6}$ Mask aligner: In contrast to wafer steppers, where a pattern of a photomask is projected onto a smaller area and a wafer is subsequently exposed, in a mask aligner a photomask can be aligned and a full-area exposure is conducted.
} 
water and a patterned hard mask remains. The resolution of these inkjet printing processes is in the range of $50 \mu \mathrm{m}$ feature sizes. Processing velocities of inkjet printing can be moderately high using modern drop-on-demand multi-nozzle printheads. An advantage for both approaches, - the laser as well as the inkjet patterning of etching masks, - is that these are contact-free patterning techniques.

Using photolithographic processes, a wide range of defined textures was investigated in several publications. In [39], a wide range of texture types based on the formation of pyramidal features when etching $<100>$ silicon in alkaline solutions is described. All these features applied to solar cell front surfaces aim for a positive effect on a broadband spectral range. Therefore, feature sizes are typically in the range of several microns. It has been shown that certain defined pyramidal texturing schemes are superior to random pyramid textures; the defined inverted pyramid texture was applied when reaching the highest efficiency on monocrystalline silicon [31]. When comparing the optical quality of random pyramids and photolithographically manufactured inverted pyramids found in the literature, it is doubtful that the small gain offered by inverted pyramids is worth the effort in industrial fabrication, even when finding a cheap substituting process for photolithography. What's more, all these processes rely on anisotropic wetchemical etching; this is not possible for mc-Si, and it is precisely for mc-Si that there is a need for optimising the optical properties as was shown in Figure 3.9. Therefore, in the following section, one concept for improving the optical properties of the front surfaces of mc-Si solar cells is described. Within the framework of the present work, nanoimprint lithography is used for the patterning of etching masks. This process allows by far higher resolutions than the above described alternative patterning techniques. Therefore, the fabrication of another, more sophisticated type of defined solar cell textures, namely diffractive gratings, is also made possible. As described before, this concept can be applied to achieve strong path length enhancements. Consequently, the state-of-the-art of diffractive gratings for wafer-based solar cell applications will also be described (with the focus on rear side grating applications.

\subsubsection{Honeycomb texturing of multicrystalline silicon}

Industrially established maskless etching processes on mc-Si yield weighted reflectance values around $25 \%$. To achieve homogeneous textures over the whole wafer area, isotropic etching processes have to be applied. These can be of acidic wet-chemical nature or plasma etching processes. Applying a defined texture analogue to the inverted pyramids to $\mathrm{mc}-\mathrm{Si}$, meaning that an etching mask with a crossed pattern symmetry and isotropic etching is applied, Stocks et al. [88] found 
that the overall reflection is only a little below the current state-of-the-art acidic texturing. However, they found light trapping can be increased drastically. To explain the moderate overall reflectivity of this textured surface, they reported that there was a considerable share of planar surface areas after the texturing. One aspect leading to planar surface areas is that the perpendicularly arranged openings in the dielectric etching mask patterned via photolithography had a finite diameter of $4 \mu \mathrm{m}$ (the pattern period was $10 \mu \mathrm{m}$ ). When applying isotropic etching processes, the unmasked areas remain planar. A second aspect is related to the pattern symmetry. To reduce the overall reflectivity, it is important to minimise planar surface areas and also to fabricate etching pits with steep sidewalls. The latter can be optimised when applying a texture with etching pits arranged with the highest packing density (hexagonal arrangement).

This so-called honeycomb texture was first demonstrated by Zhao et al. [89]. There, hole openings of again $4 \mu \mathrm{m}$ diameter were arranged hexagonally with a pattern period of $14 \mu \mathrm{m}$. A thick thermal oxide layer was patterned photolithographically and was then used as an etching mask in an acidic wetchemical etching process. Applying a double layer antireflection coating and a PERL ${ }^{7}$ cell structure, a then record efficiency for mc-Si of $19.8 \%$ was achieved. The short-circuit current density $j_{s c}$ was $38.1 \mathrm{~mA} / \mathrm{cm}^{2}$ showing the high optical quality of this texture. This is surprising, since ray-tracing simulations performed by Schultz [21] showed that this etching mask geometry in combination with purely isotropic etching leads to values for the weighted reflectance of around $20 \%$ in the best case (without ARC). Zhao et al. identified the finite hole openings achievable by photolithographic patterning as one aspect limiting the optical efficiency of this texture.

Having the identical arrangement for the etching mask, Schultz et al. investigated plasma etching processes for the pattern transfer into silicon [90]. The possibility of achieving higher aspect ratios leads to a higher optical quality close to that of inverted pyramids. They reported values for the weighted reflection of honeycomb textured mc-Si substrates is as low as $14 \%$ (without ARC). In a further work, Schultz et al. verified this very high optical quality on the cell level [5]. There, the still held record efficiency for mc-Si of $20.3 \%^{8}$ was reported. Interestingly, this record cell had a $j_{s c}$ of $37.7 \mathrm{~mA} / \mathrm{cm}^{2}$ and a cell thickness of only around $100 \mu \mathrm{m}$. The high $j_{s c}$ for this thin solar cell not only highlights the excellent reduction of overall reflectivity of this texture, but also the light trapping properties. On thicker

\footnotetext{
7 PERL: Passivated emitter and rear locally diffused cell structure [11].

8 This efficiency was later corrected to $20.4 \%$ based on the revision of the standardised solar spectrum.
} 
mc-Si wafers experiencing this processing scheme, values for $j_{s c}$ up to $39.3 \mathrm{~mA} / \mathrm{cm}^{2}$ were measured [21].

These impressive results in particular inspired research groups to find alternative processes to allow fabrication of this high-efficiency texture in industrial environments. As can be seen, there is a huge gap in weighted reflectance $R_{w}$ of state-of-the-art isotextured substrates to these honeycomb textured ones of around $25 \%$ to $14 \%$ respectively (without ARC). This justifies potential additional efforts in fabrication as considerable benefits in solar cell performance can be expected.

The inkjet patterning of etching masks for the honeycomb texturing was investigated e.g. by Fraunhofer ISE [86] as well as the University of New South Wales in Australia [87]. Both groups combined the patterning of etching masks with wet-chemical etching. Minimal values for the weighted reflectance were as low as $18.4 \%$. Solar cell efficiencies using this patterning approach were not reported up to now.

The fabrication of honeycomb textured surfaces via laser ablation was in particular pushed forward by Niinobe and co-workers from Mitsubishi Electric Corporation [84]. There, a silicon nitride layer was deposited via PECVD. This layer was opened by laser ablation and then served as an etching mask in an acidic wet-chemical etching process. Data on weighted reflectivity was not reported. However, they achieved an efficiency of $19.1 \%$ on a large area $\left(216.6 \mathrm{~cm}^{2}\right) \mathrm{mc}-\mathrm{Si}$ solar cell using a PERC cell structure. The corresponding value for $j_{s c}$ was $38.8 \mathrm{~mA} / \mathrm{cm}^{2}$ and the cell thickness was reported to be around $200 \mu \mathrm{m}$. On a $100 \mu \mathrm{m}$ thick cell they achieved $18 \%$ efficiency and $a j_{s c}$ of $38.1 \mathrm{~mA} / \mathrm{cm}^{2}$.

Very promising results for a honeycomb texturing process based on a direct laser ablation of silicon were just recently reported from the Institut für Solarenergieforschung Hameln (ISFH) [91]. They showed excellent values of $j_{s c}$ for honeycomb textured FZ c-Si substrates of up to $40.6 \mathrm{~mA} / \mathrm{cm}^{2}$ and $39.3 \mathrm{~mA} / \mathrm{cm}^{2}$ on $\mathrm{mc}-\mathrm{Si}$. However, this direct laser ablation process is very time consuming.

Two other approaches for the honeycomb texturing of mc-Si can be found in the dissertation of Li at the Massachusetts Institute of Technology [92]. There, a photodefined etching as well as a surface modification to achieve a selective wetting or de-wetting of silicon surfaces was investigated. In the first technique, the electrochemical properties of a semiconductor / electrolyte interface, which had been modified by localised illumination, were used to trigger etching processes. This technique was dismissed within that work because of a limited lateral resolution of the etching process. In the second approach, a soft lithography process is applied using PDMS stamps to selectively modify the wettability of the 
silicon surface. A polymeric resist is then applied self-aligned on areas with or without modified surface energy. Finally, a wet-chemical etching process is conducted. Within that thesis, these basic ideas are investigated, but no effective texturing process was set up.

\subsubsection{Diffractive gratings for solar cells}

Beyond the classical and well-understood relatively large front surface textures described above, diffractive features began to be investigated in the 1990s in order to enhance light trapping effects [93]. Making use of wave optically resonant effects gave rise to expressions like ultra-light-trapping or photon management. The first expression ultra-light-trapping is used for schemes that exceed the path length enhancement as imposed by the Yablonovitch limit. However, in spite of over 20 years investigation of diffractive features in solar cells, it is still undecided whether periodic diffractive features are superior to stochastic scattering textures [94]. Summing up the results of many theoretical works, the message can be extracted that if a broadband path length enhancement has to be achieved (e.g. as for silicon thin-film applications) a diffuse reflector is superior to strictly periodic features. Dealing with relatively thick silicon substrates in wafer-based applications, the situation can be different. There, only a narrow spectral range has to experience a strong path length enhancement. Thus, photonic features can be designed to be effective in this weakly absorbed spectral range. Theoretical considerations on this topic can be found in [94-96]. Besides these theoretical aspects of maximum achievable path length enhancements, there are two advantages for the realisation of periodic features in solar cells: (i) they can be fabricated in a well-defined way, which is important for understanding and characterising optical as well as electrical effects and (ii) the fabrication of the ideal Lambertian scatterer, which typically serves as reference in theoretical considerations, is yet to be realised.

In the following, the results of published experimental works are summarised. The focus is placed on diffractive gratings on the rear surface of wafer-based crystalline silicon solar cells. The main research on this topic was conducted at the Paul Scherrer Institute in Switzerland. There are countless other publications on diffractive gratings on the solar cell front or in particular for use in thin film applications. However, the boundary conditions for these embodiments are very different to those followed within this work. When integrating a diffraction grating on the front surface of a standard type solar cell, one has to deal with several topics like broadband antireflection, emitter formation, passivation and of course light trapping. For the introduction of such a feature in the solar cell rear, one can focus mainly on the light trapping and the passivation. Nonetheless, in 
principle the integration of such a grating on the front can also be very attractive as described in [95].

Early experimental work for the fabrication of diffractive features in silicon solar cells required elaborate technologies like electron-beam lithography, which is only suitable for small area processing, or interference lithography [97, 98]. Kies and co-workers described in [97] the fabrication of diffraction gratings on the wafer rear side with periods of $310 \mathrm{~nm}$ and $620 \mathrm{~nm}$ via interference lithography and subsequent plasma etching. The smaller periods are designed such that for the target wavelengths only the \pm first diffraction orders propagate and these orders propagate in oblique angles. In [50], the same group at the Paul Scherrer Institute published optical results of solar cell precursors. This time they focused on larger periods, but investigated also the possibility of introducing blazed gratings on the wafer rear. They found that the smaller periods are inferior in terms of light trapping and impose tighter process windows in terms of the grating depths to be realised. As in the previous works, they fabricated solely linear diffraction gratings. They found that the blazed grating performs better than the binary one (for a planar front surface) and described that the effective thickness of a wafer with the linear blazed grating can be enhanced by a factor of the order of five.

Besides these issues, two other important aspects when introducing diffraction gratings on the solar cell rear were also highlighted by this group in [98]: (i) the metallisation on top of the corrugated grating surfaces on the solar cell rear gives rise to parasitic absorption, which has to be minimised; (ii) the introduction of a grating in the silicon bulk deteriorates the electrical properties of this interface. For the first aspect, they mainly focused on the fabrication of smooth surfaces for the grating's topography and thus minimise rough surfaces as sub-topography. Furthermore, they proposed the use of silver for the rear metallisation to minimise parasitic absorption. However, the problem of having a corrugated metal surface in general is not captured as a problem to be solved. For the second aspect of the structured rear interface, they proposed a decoupling of the electrically active interface from the optically active grating layer. This is realised by applying a thin thermal oxide layer on the planar wafer. Onto this passivating oxide layer, an amorphous silicon layer is deposited, which then is patterned. In [98] an enhanced external quantum efficiency (EQE) for a $40 \mu \mathrm{m}$ thick silicon solar cell is measured. There, the grating is wet-chemically etched into the silicon bulk, the surface is passivated by $5 \mathrm{~nm}$ silicon oxide and silver is applied as the rear contact. Despite showing an enhanced EQE, they also measured considerable parasitic absorption. What was very positive about their findings is that they did not see any increase in rear surface recombination velocity $\left(S_{\text {back }}\right)$ in the internal quantum efficiency. This is probably related to the smooth wet-chemical etching process, since in the same 
publication they reported a deteriorated $S_{\text {back }}$ when they applied ion beam etching. Another very interesting aspect already conceived in this early stage is related to up-scaling of manufacturing processes to fabricate diffractive gratings in solar cells. They contemplated embossing processes as a potential solution to allow industrially feasible processes. These processes can be seen as predecessors to the ones investigated within this thesis.

Beyond these works at the Paul Scherrer Institute, there was activity related to the simulation of diffractive features on the rear of wafer based silicon solar cells [64, 95, 96, 99]. As an additional result to the ones described before, Mellor showed that bi-periodic grating symmetries are superior to linear gratings [95]. Furthermore, these theoretical works more or less in agree that the optimal periods are larger than the $620 \mathrm{~nm}$ investigated by Morf et al. and are rather situated around $1 \mu \mathrm{m}$, depending on the wafer thickness. While most of these works investigate binary or pyramidal patterns, Gjessing investigated rather complex structures, which can be somehow described as 2D blazed gratings [96]. Analogue to the experimental results on blazed gratings in the 1D case by Morf et al., these so-called zigzag patterns are superior to binary gratings in the 2D case. However, the feasibility of practical realization was not investigated up to now.

An alternative approach for the fabrication of diffractive elements on the wafer rear side is based on self-assembly processes when applying solutions of monodisperse spheres [100]. There, a spin coating process was applied with the aim of realising a single layer of silicon oxide or PMMA spheres. Afterwards, this pattern was infiltrated by a high refractive index silicon carbide material. In this study, a path length enhancement was verified on the cell level. However, there was an issue with a degraded $S_{\text {back }}$ counterbalancing this effect. The technology of spin coating itself is relatively simple; however, template based processes allow more degrees of freedom. 


\subsection{Electrical effects of textured surfaces}

In texturing a silicon solar cell surface, one aims to increase the conversion efficiency of this system. However, besides the optical gain that can be achieved by this texturing negative effects also occur. These are related to the electrical properties of the modified semiconductor. In this section, first some general considerations on mechanisms leading to the diminished electrical properties will be explained. To this end, the discussion will be focused on front side textures. The conclusions drawn from them can be translated to rear side textures. Afterwards, some experimental results will be reviewed to assess different texturing technologies, processes and resulting shapes.

\subsubsection{General considerations - a review of studies on alkaline etching of monocrystalline silicon}

In section 2.2, the basic recombination mechanisms in silicon were explained. was shown that the surface recombination rate is proportional to the surface area of the semiconductor [8]. This effect directly implies that when texturing a silicon surface, the overall surface recombination will increase. But this is not the only effect leading to higher surface recombination velocities $S$. In the following, some publications will be reviewed to understand the relevant effects as best as possible. These studies are based on the use of well-defined monocrystalline material. Besides the high material quality, which is necessary to allow a reliable investigation, another advantage is that a very defined and discrete texturing can be ensured. Texturing $<100>$ c-Si wafers in alkaline solutions leads to pyramidal textures as a result of the intersection of $<111>$ planes. Thereby a discrete surface enlargement of a factor of 1.73 compared to a flat surface can be realised. As the alkaline wet-chemical etching process leads to very smooth surfaces, it can be assumed that this process does not overly influence the actual behaviour and that this is rather determined by material properties.

In equation (2.10), which describes the surface recombination rate, the input parameters other than the surface area were e.g. defect densities. When passivating the surface by saturating dangling bonds, one aims for a reduction of these defect densities. As was also shown in chapter 2, the atomic packing density is different for different crystal orientations. The atomic spacing in $<100>$ planes is a factor of $\sqrt{2}$ larger than in $<111>$ planes. Therefore, it can be assumed that the density of defects should be different for different crystal planes. This is confirmed by measurements conducted by Jin et al. [101]. They investigated non-diffused and diffused planar $<100>$ and $<111>$ as well as textured $<111>$ surfaces passivated 
by a thermal oxide. Besides finding a higher defect density in $<111>$ silicon, they additionally assume a higher defect recombination activity on such interfaces.

Diffusing emitters in wafers with textured surfaces and/or different crystal orientations further complicates the situation. Glunz showed in [102] that especially lightly diffused deep emitters on inverted pyramid textures reveal surprisingly high emitter saturation current densities $j_{0 e}$. As a potential reason for this effect, an inhomogeneous diffusion profile as a result of the 3D pyramidal texture is discussed. It was stated that higher doping concentrations could result on the tips of the texture than at the bottom of the pyramids. However, he then also measured values for $S$ without applying an emitter diffusion on oxidepassivated samples and found values of $67 \mathrm{~cm} / \mathrm{s}$ and $1200 \mathrm{~cm} / \mathrm{s}$ for the planar and the textured sample respectively, which cannot be explained by a surface enlargement.

Recart and Cuevas [103] conducted capacitance measurements to investigate the enlargement of the junction area induced by a surface texture. They concluded that this effect especially comes into play for large features and could be used to explain enhanced recombination in the space charge region.

A study focused on the effects induced by thermal silicon oxide passivation of textured surfaces was performed by Cousins and Cotter [104]. They considered the stress induced during the film growth of the thermal oxide as a potential source of degraded passivation quality of textured silicon surfaces. To verify this effect, they demonstrated an improved behaviour when rounding sharp edges in a short acidic etching process after the alkaline texture formation.

A review on this topic was conducted by McIntosh and Johnson [105]. In terms of surface recombination velocities on non-diffused passivated samples, they report on a wide range of measured values by different groups. For samples passivated by a thermal silicon oxide, the ratio of $S$ of textured to planar wafers varied between 2 and 4 . For samples passivated by silicon nitride this ratio varied between 1.5 and 35. This huge discrepancy in the latter case is probably related to the different passivation field-effect mechanism and a difference in fixed charge densities. For diffused samples, carrying together data from the works of several groups, they confirm the findings of Glunz [102] that the $j_{0 e}$ enhancement of textured to planar samples depends strongly on the peak doping concentration and the sheet resistance of emitters. This makes sense, as the passivation quality of lower doped emitters is more effective, but also because, in a highly doped emitter, a shielding of the surface can be achieved and Auger recombination dominates. This effect is visualised in Figure 3.12 using the result of a rudimentary PC1D simulation, for which high quality $p$-type FZ material and a constant junction depth of $1 \mu \mathrm{m}$ of the 
Gaussian shaped phosphorous emitter were assumed. Then, a variation on the peak doping concentration as well as the front surface recombination was conducted (no rear surface recombination was considered). Using the results of $V_{o c}$ and $j_{s c}$ the dark saturation current density was calculated using the one diode equation. It can be seen that, for very high doping concentrations, the surface recombination velocity has virtually no influence on the dark saturation current density $j_{0}$.

To sum up the results of the review [105], a $2-12$ times higher recombination is found for lightly doped or non-diffused samples passivated by silicon oxide. This is by far higher than the surface enlargement alone. A second reason is the higher defect density of $<111>$ compared to $<100>$ planes. Further on, capacitancevoltage measurements showed that the defect density of planar $<111>$ surfaces is lower than for textured $<111>$ surfaces. It is argued that stress induced by film growth is responsible for this increased defect density. However, this was not resolved fully.

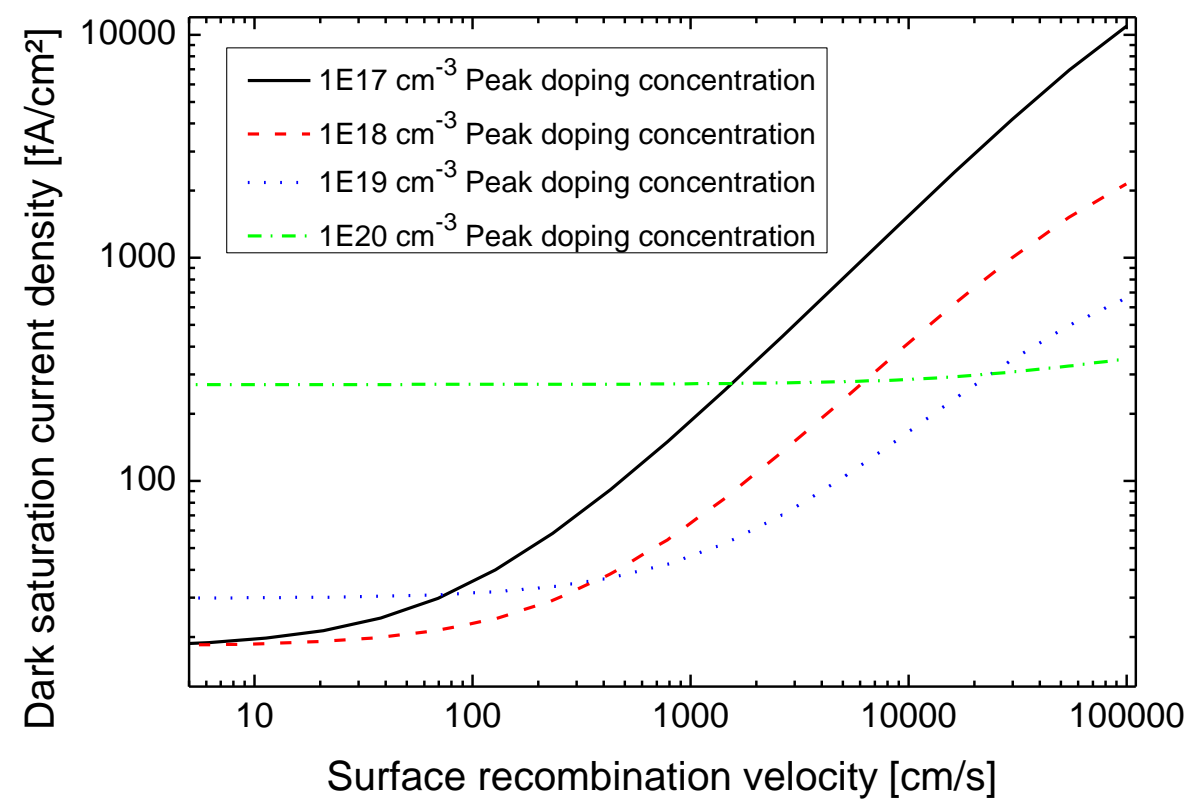

Figure 3.12: Simplified PC1D simulation to visualise the interacting influence of front surface recombination velocity and peak doping density on the dark saturation current density. The doping depth was kept constant (junction depth $1 \mu \mathrm{m}$, Gaussian emitter, p-type $1.5 \Omega \mathrm{cm}$ base resistivity, 1 ms minority carrier lifetime).

Results of a similar model implemented in PC1D are shown in Figure 3.13. There, the effect of either $S_{\text {front }}$ or $S_{\text {back }}$ on the open-circuit voltage for a cell with exemplary PERC parameters is studied. The non-diffused rear surface is passivated and thus the dark saturation current density is very sensitive to increases of $S_{\text {back }}$. 
Therefore, the impact of $S_{\text {back }}$ on $V_{o c}$ is typically higher than that of $S_{\text {front }}$ for this cell structure. A conclusion that can be drawn from these simulations is that, for PERC type solar cells, the possible increase in rear surface recombination velocity $S_{\text {back }}$ caused by a surface textured is particularly crucial.

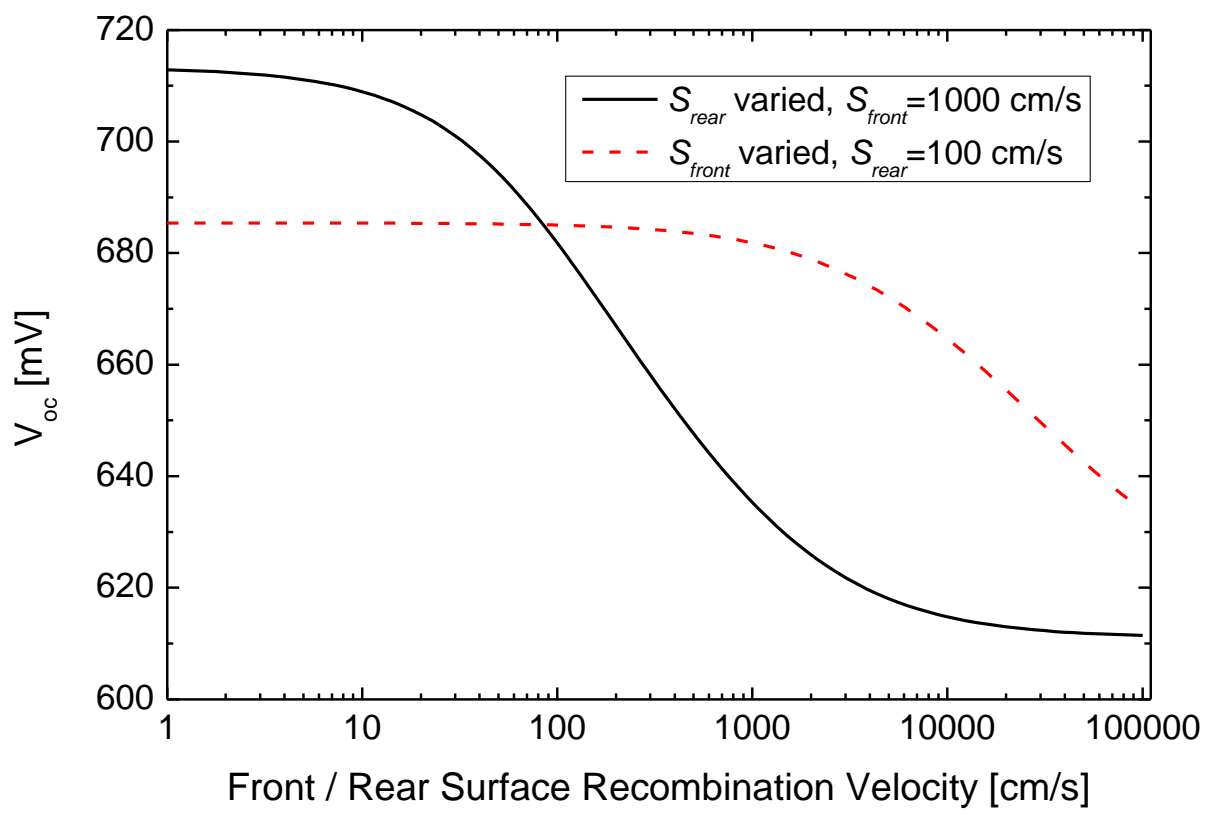

Figure 3.13: Results of PC1D simulations to visualise the difference in the effect of front or rear surface recombination velocity on the open-circuit voltage exemplarily for a PERC cell structure. When keeping $S_{\text {front }}$ constant a higher value was chosen, as the passivation quality typically depends on the dopant density [15]. Other cell parameters: p-type $1.5 \Omega \mathrm{cm}$ base resistivity, $1 \mathrm{~ms}$ minority carrier lifetime, peak doping $1 E 19 \mathrm{~cm}^{-3}$, junction depth $1 \mu \mathrm{m}$, Gaussian emitter.

A more recent study published by Baker-Finch and McIntosh [106] is about the distinguishing of $\langle 111\rangle$ facets in textures or edges and vertices as sources of decreased passivation quality. To investigate this influence they fabricated samples textured with inverted pyramids and varied their period. Thereby, the density of edges and vertices can be changed while keeping the surface enlargement constant. Furthermore, in this study, they investigated both silicon oxide as well as silicon nitride for the passivation. In a first step, they found for both passivation schemes on FZ $n$-type material a minimum for $S$ for a period around $10 \mu \mathrm{m}$. While for silicon oxide the minimum was rather flat, for silicon nitride there was a sharp minimum. This behaviour might be seen as contradictory to the conclusion of Cousins [104] that stress at sharp corners is responsible for increased values of $S$. The fact that values of $S$ increase towards smaller features is in agreement with this conclusion; however, for larger features, the density of sharp features is decreasing and thus $S$ should also be steadily decreasing, which was not found to 
occur. Baker-Finch and Mclntosh could not confirm experimentally that stress induced by thermal oxidation is the reason for this degradation.

From all these findings it can be concluded that texturing of a non-diffused $<100>$ c-Si surface leads to an increased surface recombination velocity due to (i) an enlarged surface; (ii) the exposure of crystallographic orientations with higher defect densities; and (iii) a dependency on feature periods; however, the responsible physical mechanisms (stress, enhanced defect density on edges, geometric effects, etc.) are yet to be fully clarified.

Forming an emitter by diffusion processes, the recombination described by the emitter dark saturation current density $j_{0 e}$ not only depends on $S$, but also strongly on the doping profile. High peak doping densities make $j_{0 e}$ insensitive to changes in $S$. For high-efficiency type emitters (low peak doping and deep junction depth) the $j_{0 e}$ of textured samples found in the literature was surprisingly high compared to planar references. This high ratio cannot be fully understood by reviewing the works listed in this section.

The effects were mainly discussed in the context of front side textures. The findings in principle can be directly transferred to rear surface textures. It was shown that, in particular for PERC type solar cells, the open-circuit voltage is strongly influenced by the $S_{\text {back }}$.

\subsubsection{Effects implied by other texturing processes on multicrystalline silicon}

The situation for mc-Si is different to the one sketched before for c-Si. First, there is no unique crystal orientation. The standard texturing scheme for this material is therefore independent of crystal orientations. When choosing the etching duration for the isotexture, a trade-off must be sought between reflectivity and surface recombination velocity $S$ [107]. Removing only a little material allows the realisation of optically good textures, but $S$ is found to be moderate. When removing more material, the surface becomes more planarised, and thus the optical quality decreases; however, the electrical quality improves and thus higher values for $V_{o c}$ can be achieved. Removing around $5 \mu \mathrm{m}$ leads to an optimal tradeoff between these effects [40].

In a recent publication, Baker-Finch et al. investigated the recombination effects of isotextured surfaces and their implications on cell efficiencies [108]. They find that the surface enlargement seems to be a minor factor regarding $S$ (for surfaces passivated with hydrogenated $\mathrm{SiO}_{2}$ or silicon nitride). This study is performed on $\mathrm{Cz}$ material on which varying depths are removed in an acidic etching process. The optimum in etching depth is determined to be $1-3 \mu \mathrm{m}$. They claim that this smaller removed volume compared to the older study mentioned before might be 
related to improvements in the wire sawing technology. In a following study, they report on multicrystalline silicon solar cells fabricated in an industrial environment with different amounts of silicon removals [109]. Their experimental results are in agreement with the older results of A. Hauser et al. [40] showing that an optimal removal of silicon is around $5 \mu \mathrm{m}$.

Still it seems not fully clear if the reason for this optimum pattern depth is mainly related to a removal of damaged area. Baker-Finch et al. stated that they did not see any dominating influences of varying crystal orientations or enlarged surfaces. However, in the very beginning, there are very sharp, convex and concave, features that besides the sawing damage could be responsible for high values of $S$. Alternative textures like the formation of porous silicon or needle-like black silicon surfaces show excellent optical properties; however, the electrical properties of these nano-scale textures seem to be of low quality. Both of these features introduce a highly enlarged surface area into silicon wafers. Although these textures exhibit different attributes that might be responsible for the respective diminished passivation qualities, the published results show similar characteristics. The front surface reflection is very small, but small values for short circuit current density were reported $[73,110]$. Also similar is the typically low blue response of devices with such front surface textures, indicating high surface recombination.

However, the formation of these nano-scaled features is totally different. In the case of black silicon fabrication, reactive ion etching processes are involved. The related ion bombardment and radiation can induce damages in the silicon crystal [111]. These plasma process related effects will be described in more detail in section 4.4. Besides these plasma induced damages, critical aspects for the black silicon texture are: (i) dopant distribution after emitter diffusion; (ii) small, needlelike features are oxidised immediately and thus will be removed in any HFcontaining cleaning step; (iii) mechanical stability in the following process steps. The situation for the porous silicon layers is different; an electrochemical etching process is applied for the formation the porous layer. This is not believed to introduce any damages in the underlying silicon crystals. Furthermore, mechanical stability does also not seem to be an issue for this type of surface texture. However, the problem of very high values of $S$ seems to be similar to the black silicon textures.

The black silicon texturing experienced a revival recently, which has to do with the appearance of the deposition technology of atomic layer deposition (ALD). This technology allows very conformal layer deposition and, especially for the case of aluminium oxide, a very effective surface passivation for silicon solar cells. Using this technology, very low values for $S$ on black silicon textured silicon wafers as 
low as $7 \mathrm{~cm} / \mathrm{s}$ were measured [112]. The best results published of black silicon textured FZ c-Si solar cells with diffused emitters still indicate that this texture is until now not suitable for high-efficiency processing [113].

\subsection{Summary and assessment of the approaches}

The findings of this chapter are summarised in Table 3.1, where different front side textures are compared in terms of reduction of the front surface reflection, light trapping and passivation quality. Furthermore, published data on weighted reflectances is listed. A very similar comparison can be found in [21] and [114].

Table 3.1: Summary of front surface textures. The different approaches are assessed in terms of reduction of front surface reflectivity (without $A R C$ ), light trapping and passivation quality. ++ = good $;+=$ moderate; 0 = bad .

\begin{tabular}{|c|c|c|c|c|c|c|}
\hline Texture type & $\begin{array}{l}\text { Front surface } \\
\text { reflection }\end{array}$ & $\begin{array}{l}\text { Light } \\
\text { Trapping }\end{array}$ & $\begin{array}{l}\mathbf{R}_{\mathrm{w}} \\
{[\%]}\end{array}$ & Ref. & $\begin{array}{c}\text { Passivation } \\
\text { Quality }\end{array}$ & Comment \\
\hline $\begin{array}{l}\text { Random } \\
\text { pyramids }\end{array}$ & ++ & ++ & $\approx 11-12$ & $\begin{array}{l}{[69],} \\
{[115]}\end{array}$ & ++ & \\
\hline $\begin{array}{l}\text { Inverted } \\
\text { pyramids }\end{array}$ & ++ & ++ & $\approx 10$ & {$[11]$} & ++ & Needs lithography \\
\hline Isotexture & + & + & $\approx 23$ & {$[40]$} & + & \\
\hline $\begin{array}{l}\text { Mechanical } \\
\text { micromachining }\end{array}$ & ++ & ++ & $\approx 14$ & [116] & + & $\begin{array}{l}\text { Damage removal; } \\
\text { critical for thin wafers }\end{array}$ \\
\hline $\begin{array}{l}\text { Direct laser } \\
\text { writing }\end{array}$ & + & + & $\approx 16$ & [114] & & Damage removal \\
\hline $\begin{array}{l}\text { Honeycombs } \\
\text { (wet-chemical } \\
\text { etching) }\end{array}$ & + & + & $\approx 20$ & $\begin{array}{l}{[21],} \\
{[89]}\end{array}$ & ++ & Needs lithography \\
\hline $\begin{array}{l}\text { Honeycombs } \\
\text { (plasma etching) }\end{array}$ & ++ & ++ & $\approx 14$ & $\begin{array}{l}{[90],} \\
{[21]}\end{array}$ & ++ & Needs lithography \\
\hline Porous silicon & ++ & 0 & $\approx 6$ & [117] & 0 & \\
\hline Black silicon & ++ & $0(+)$ & $\approx 2$ & [110] & 0 & \\
\hline
\end{tabular}

The pyramidal texturing of $\mathrm{c}-\mathrm{Si}$ already leads to very good optical properties when being applied stochastically. Thus, the additional lithographic effort to realise inverted pyramids will probably not be worthwhile for industrial fabrication as 
there will be no considerable benefit. The nano-scale textures, porous as well as black silicon, allow an excellent reduction of the front surface reflectivity; however, due to the very small features, the light trapping is not believed to be effective. Furthermore, the electrical properties that can be extracted from the literature indicate that recombination effects cannot be suppressed effectively, particularly for diffused samples.

Both mechanical abrasion as well as direct laser ablation lead to optically good textures in terms of reduction of the front surface reflectivity as well as light trapping. However, these technologies have to be combined with subsequent wetchemical etching to remove damaged areas, processing times are relatively high, resolution is limited and, in the case of mechanical abrasion, wear of the tooling has to be considered.

Trying to achieve similar optical behaviour to pyramidal textures on c-Si for mc-Si (broad band suppression of front surface reflection and light trapping via refraction), so far the best results were achieved using the honeycomb texture. There, the potential gain compared to the state-of-the-art isotexture is high, so that additional effort can be tolerated. In principle, both direct as well as lithographic patterning can be applied for the realisation of this type of texture. As a result of this consideration, the applicability of nanoimprint lithography (NIL) for the fabrication of honeycomb textured surfaces is investigated within this work as will be described in section 5.1.

The technology of NIL allows the patterning of etching masks with very high resolutions. Therefore, it is possible to realise diffraction gratings as described in section 3.2.2. There, it was reported that most of the experimental work found in literature is focused on the realisation of rear side gratings. This approach was also followed within this thesis. As was shown in this chapter, introducing textures onto the rear side can be very critical, especially for PERC type solar cells, since they are very sensitive to deteriorations of $S_{\text {back }}$. In the works of Morf and coworkers [98], it was suggested that an additive patterning in terms of applying an optically matched patterned layer on the planar passivated rear side might be a solution to overcome this problem. This additive approach is in particular suited for the wafer rear side since on the front care has to be taken to avoid parasitic absorption. 


\section{Texturing scheme developed within this work}

In this chapter the process chain developed within this work for an industrial realisation of defined textures will be presented. The focus will be placed on the development of tools and processes for nanoimprint lithography tailored for the demands set by PV applications. In the following sections, the individual technologies of interference lithography (master origination), cast moulding or hot embossing (stamp fabrication), nanoimprint lithography (patterning of an etching mask) and plasma etching (pattern transfer into the substrate) will be described in more detail.

\subsection{Overview of the developed process chain}

The fabrication of distinct patterns can either be realised by top-down or bottomup approaches. Bottom-up approaches make use of self-assembly processes, which typically occur on the molecular scale (e.g. in the formation of hexagonal pores for anodic alumina [118]) or in the micro- and nano-scale by self-arranging processes (e. g. the formation of random pyramids on c-Si [69], the origination of opaline structures by sedimentation [119] or by spin coating of mono-disperse spheres $[100,120])$. In top-down approaches, a defined pattern is applied to a layer, for example in lithographic processes as developed for microelectronics. Topdown processes can be further divided into sequential direct writing processes (e.g. direct laser ablation of silicon [121]) and template based processes. These well-defined processes are typically used for the realisation of high-efficiency solar cell textures. In section 3.2, an overview of typically applied processes for the realisation of defined textures on silicon solar cells was given. Table 4.1 summarises some of their main characteristics as well as those of nanoimprint lithography (NIL) relevant for solar cell texturisation. 
Table 4.1: Summary and qualitative assessment of technologies allowing a defined texturing (either directly or via subsequent etching processes). Question marks for NIL indicate aspects to be addressed within this thesis. $++=$ very good; $+=$ good; $0=$ medium; $-=$ moderate; $--=$ bad.

\begin{tabular}{lcccc}
\hline Process & Resolution & Throughput & $\begin{array}{r}\text { Requirements } \\
\text { on substrate } / \\
\text { pre-treatment }\end{array}$ & $\begin{array}{c}\text { Suited for large } \\
\text { area solar cell } \\
\text { processing }\end{array}$ \\
\hline Screen-printing & -- & + & ++ & ++ \\
Inkjet & - & + & + & + \\
$\begin{array}{l}\text { Direct laser } \\
\text { ablation }\end{array}$ & 0 & -- & + & - \\
$\begin{array}{l}\text { Laser ablation of } \\
\text { etching masks }\end{array}$ & 0 & 0 & 0 & - \\
$\begin{array}{l}\text { Photolithography } \\
\text { Nanoimprint }\end{array}$ & + & - & -- & $?$ \\
lithography & ++ & $?$ & $?$ & \\
\hline \hline
\end{tabular}

In terms of resolution, NIL is superior even to photolithography and is outplaying all other processes by far. However, there are some open questions for the use of NIL for silicon solar cell texturisation indicated by the question marks in Table 4.1. These points are in principle demonstrated for special types of NIL; however, there is a demand for research into the application of this technology for solar cell texturisation. Throughput could be excellent, considering roll-to-roll imprint processes on flexible foils, but the use of Roller-NIL processes on thin, rigid and brittle substrates has not been investigated so far. In terms of the requirements on the surface quality of substrates to be processed, it is known that soft stamp materials can adapt to wavy surfaces $[122,123]$. This is essential to even allow large area processing at all. However, how this process behaves on typically very rough surfaces (in the extreme case wire-sawn multicrystalline silicon substrates) has until now been unknown. Last but not least, no publications on the use of this technology for silicon solar cell processing were known to the author of this thesis (let alone for large area processing in silicon PV). Summing up these points, it can be concluded that NIL might offer completely new possibilities for solar cell processing, but some crucial issues have to be addressed.

$\mathrm{NIL}$ is a process to pattern etching masks. Therefore, this process can only be one single step within a process chain to texture silicon wafers. First, a master structure is needed from which the stamp for NIL is replicated. After the NIL process, the 
pattern has to be transferred into the silicon via etching processes. Figure 4.1 shows a selection of possible processes that might be applicable in combination with NIL. Beyond this process chain for texturisation, the integration into the solar cell fabrication has also to be considered.

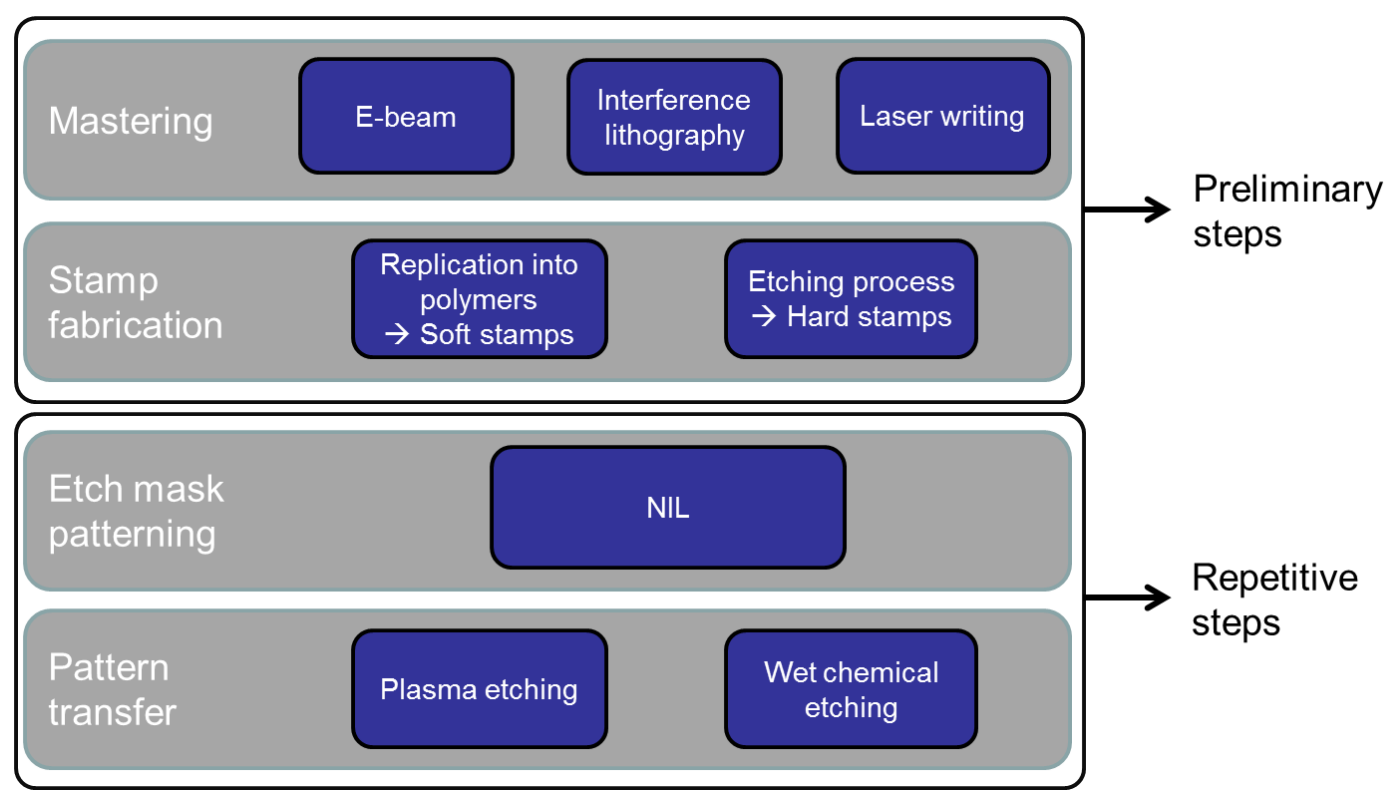

Figure 4.1: Sketch of the process chain for solar cell texturisation based on NIL developed in this work. The process chain can be divided into two stages: the preliminary steps for the stamp fabrication and the repetitive steps for patterning the silicon substrates. Some possible technologies are listed for each stage.

Interference lithography was chosen as the mastering technology within this work, because it allows the origination of surface reliefs with a wide range of feature sizes from $100 \mathrm{~nm}$ to $100 \mu \mathrm{m}$ on large areas [124]. This technology is particularly suited for periodic features, as are dealt with in chapter 5 , but can also be used to achieve non-periodic patterns. Different materials are applicable for the stamp fabrication. To allow a processing of large areas on potentially rough substrates, soft stamp materials are necessary. The pattern transfer can either be fulfilled dryor wet-chemically. We have chosen dry plasma etching processes, since they can realise a wider range of pattern geometries and aspect ratios in the silicon surface compared to wet-chemical etching processes. Finally, this texturing process flow has to be embedded into the solar cell fabrication scheme. Depending on the cell architecture, there are different options as to which step in the processing sequence the texturing is placed. As explained in section 2.3.3, the realisation of photon management structures is particularly interesting in combination with dielectric rear side passivations (PERC structure). 


\section{Declaration of main R\&D focuses of this thesis}

Most of the effort of this work was related to the development of tools and processes for nanoimprint lithography, which are tailored for the requirements set by PV applications. First, a conventional nanoimprint tool based on a planar stamp setup was developed (section 4.3.1) to gather information and learn about processing of different resist materials, plasma etching, the implementation into a solar cell process chain and potential up-scaling issues. The gained knowledge was then applied to the development of a Roller-NIL tool to enable the in-line patterning of high-resolution UV-curing etching masks on large areas on thin, brittle and opaque silicon substrates. A Roller-NIL tool fulfilling these aspects was not state-of-the-art and therefore had to be developed from scratch as described in section 4.3.4.

The main task regarding the processes of interference lithography and plasma etching was related to their integration into a complex process chain. When combining single processes into a functioning process chain, all cross-linking influences have to be understood and considered. Figure 4.2 captures schematically the interrelated process chain as well as some issues concerning interactions of the applied technologies. In chapter 4, these research topics are addressed in a general way and are revisited in chapter 5 for concrete applications, showing that different feature dimensions and surface qualities of substrates additionally change the requirements.

In section 4.2, interference lithography is presented as a mastering technology. Challenges in stamp fabrication are first described in section 4.3.2 and are revisited more precisely for the replication of very small features in section 5.2.2. Imprint processes based on different resist systems (solvent-based and solvent-free) are studied in section 4.3.3. These results, extracted using the conventional NIL tool, are then used to set up processes for the Roller-NIL tool in the best possible way. Issues regarding silicon etching processes (mainly based on polymeric etching masks) are described in section 4.4. The integral view of this whole process chain will later be described when it comes to the realisation of discrete texturing concepts (chapter 5). At that point, the embedding into the solar cell fabrication will also be considered and the feedback between single processes will be more concrete. 


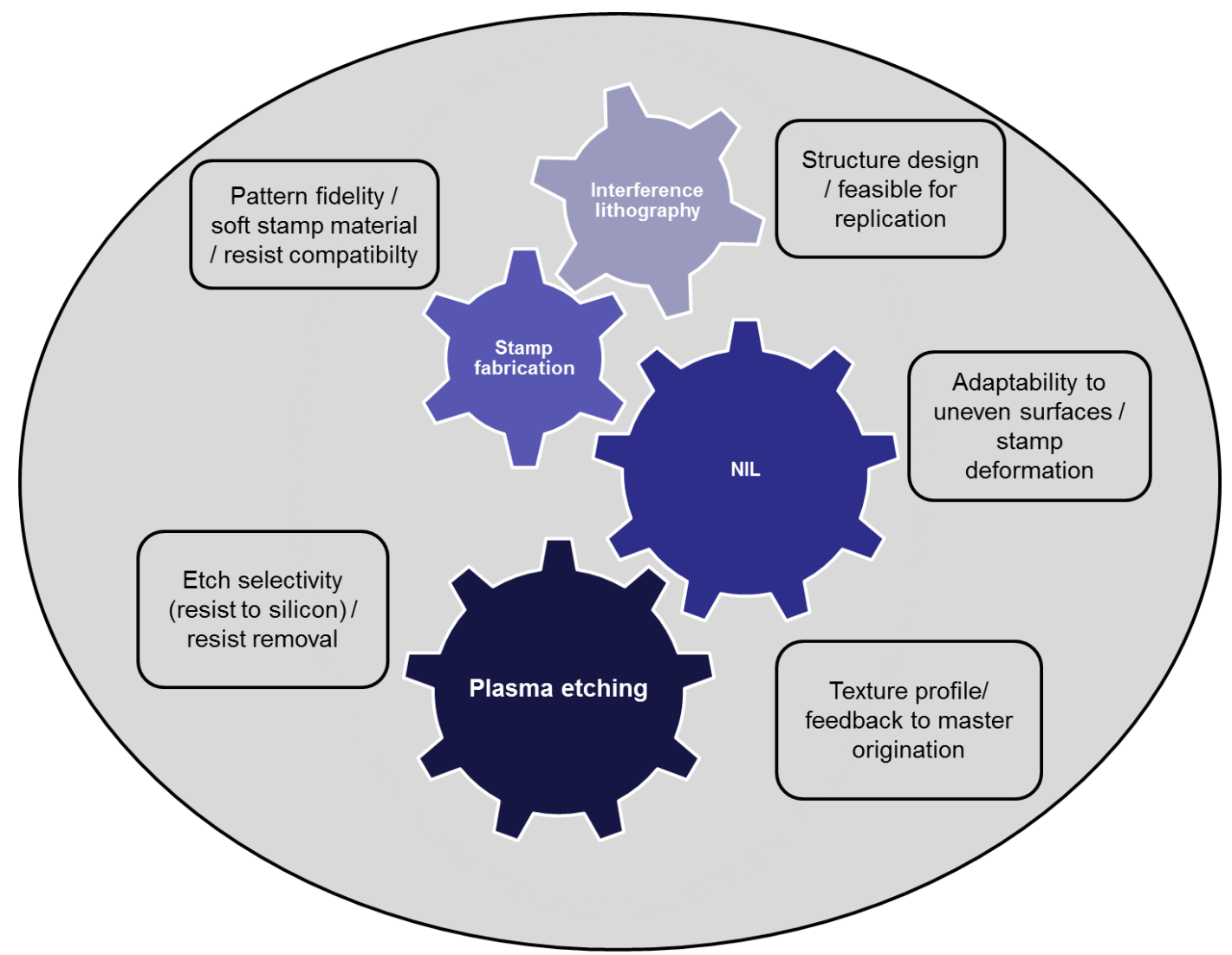

Figure 4.2: Schematic illustration of the complex process chain for silicon texturisation via nanoimprint lithography. In the boxes, important research topics are captured, which are especially relevant to consider when combining these processes into one functioning process chain.

\subsection{Master origination via interference lithography}

Interference lithography is an elegant technology to seamlessly pattern large areas with a wide range of possible feature sizes. In this technology, an interference pattern is used to expose a photoresist, which is thereby partially chemically modified as in classical photolithography processes. After the exposure, a development process is conducted in which, depending on the type of photoresist (within this work positive tone resists were applied for interference lithography), regions of high or low exposure dose are removed. The term interference implies some requirements concerning the light source for this optical lithographic technology. Interference occurs for the superposition of coherent waves, meaning that they have a constant phase relationship at each point of superposition. To meet this requirement a laser is typically used as light source.

One basic setup for interference lithography is sketched in Figure 4.3 [125]. We use an argon ion laser, which is twofold ionised and emits light of a wavelength of $363.8 \mathrm{~nm}$. The coherence length of the light emitted by such a laser is in the range 
of several metres. This has to be taken into account when designing a setup as sketched in Figure 4.3. It has to be assured that the difference in optical path lengths for the two beams is smaller than this coherence length. The laser beam is divided into two using a beam splitter and afterwards the two beams are deflected by mirrors onto the photoresist coated sample. In the optical path between the last mirror and the sample plane, a lens and spatial filter are inserted; the lens is used to expand a beam and the spatial filter is needed to remove disturbances in the wave field (e.g. caused by scattering at dust particles).

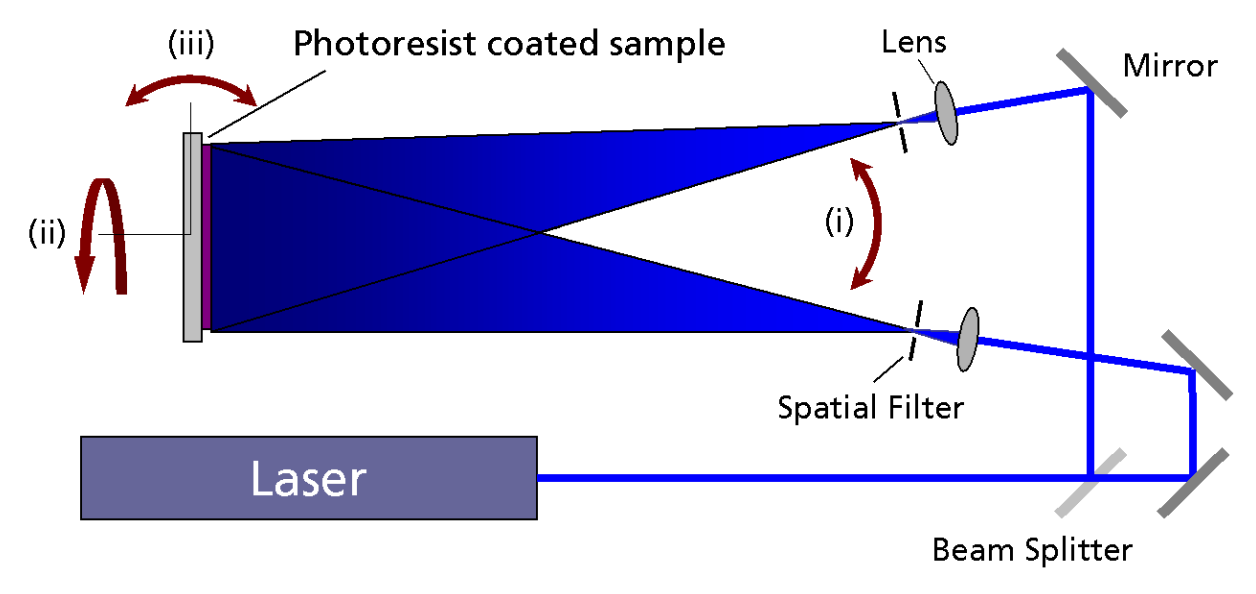

Figure 4.3: Sketch of a basic setup for interference lithography. Three degrees of freedom for the origination of periodic patterns are indicated. (i) concerns the angle of incidence $\alpha$ by which the period of the linear pattern can be adjusted. (ii) visualises the possibility of a rotation in between two exposures e.g. to generate crossed gratings. (iii): By tilting the sample prismatic profiles can be originated.

When these two expanded beams are superposed on the sample and the difference of the optical path lengths of both beams is less than the coherence length, a linear interference pattern results. The period of this pattern $\Lambda$ is determined by the laser's wavelength $\lambda$ and the angle of incidence $\alpha$ as described in [125].

Besides the angle of incidence, there are several degrees of freedom allowing the realisation of complex patterns and structure shapes as well as non-periodic patterns [58]. Two or even three dimensional modulated patterns can be originated by multiple exposures with a rotation of the sample in between or making use of interference patterns of three or four expanded beams [126]. For hexagonal patterns, as used for the honeycomb texturing in section 5.1, the superposition of three beams is applied. The corresponding setup, as well as the calculation of the dependency of the resulting period on the angles of incidence, is described in [127]. 
Using equation (4.1), periodic intensity distributions for the interference of multiple waves can be calculated. The electromagnetic field $\Psi_{i}$ describes a spherical wave (laser beam with the focal point of the lens in Figure 4.3 as a point source). The intensity distribution $I(x, y)$ of the interference pattern for the interference of $i$ waves at each point $(x, y)$ of the sample is proportional to the square of the absolute value of the electromagnetic field.

$$
I(x, y) \propto\left|\sum_{i} \Psi_{i}\right|^{2} .
$$

The original laser beam has a Gaussian intensity profile. This is only slightly modulated throughout the optical setup. Optical elements to realise top-hat intensity profiles are not applicable for their disturbance of the phase relation. Thus, the non-uniform intensity profile influences the homogeneity of the resulting pattern [58].

Up to now, we have only considered interference patterns as a result of the superposition of laser beams. However, these patterns are not transferable one-toone to the resulting surface relief in the photoresist after the development process. The resulting photoresist relief, even for identical exposure settings, depends on a manifold of parameters such as:

- reflection effects leading to losses in the deposited energy (air / resist interface) and standing wave effects as a result of the coherent light source (resist / substrate interface) [128]

- type, sensitivity and absorption of the applied resist (for $\mathrm{DNQ}^{9}$ type photoresists also the humidity is relevant for the photochemical reaction) [130]

- concentration, temperature of the developer solution as well as duration [128]

Each resist requires a specific preconditioning such as the application of an adhesion promoter, softbake parameters or re-hydration time. Another characteristic for choosing the right resist is its absorption behaviour and its sensitivity, which can be described by differential equations using the model of Dill. In this model, three parameters are used to quantify the absorption in the initial and in a bleached condition (a bleaching occurs due to the photochemical reaction) as well as the kinetics of the photochemical reaction. A description of Dill's model can be found in [131].

${ }^{9}$ DNQ: Diazonaphtochinone, a photoactive component for positive tone photoresists, typically embedded in a novolak resin [129]. 
After the exposure step, in which the resist is partially chemically modified, the development process follows, which allows further possibilities for manipulating the resulting pattern. By changing process parameters such as dilution or temperature of the developer, the development time and the preliminary baking parameters of the resist, the kinetics of the development process can be widely varied. Of particular importance is the ratio of development rate to dark erosion rate, by which resulting pattern shapes can be altered drastically.

All these points on the one hand lead to an extremely complex process around the actual interference lithography exposure step, in which there are a lot of interactions between all single steps, but on the other hand open up even more possibilities to realise nearly arbitrary pattern shapes.

The whole interference lithography process itself is rather elaborate. To compensate the high initial costs for this mastering process, high throughput replication processes have to follow. One route to allow many replications of one photoresist master is based on the origination of a metallic replication, which can then be replicated into polymers in processes nearly free of wear. In a first step, a thin conducting layer can be deposited on the photoresist master via sputtering. The metallic layer is then thickened in an electroplating process [83]. This way, whole family trees of so-called shims can be replicated out of one photoresist master. These shims (typically made of nickel) can then be applied in moulding processes like hot embossing, injection moulding or used again as a master structure for a stamp fabrication for nanoimprint lithography as described in the following subsection.

\subsection{Nanoimprint lithography (NIL)}

The basic principle of nanoimprint lithography (NIL) is not very different to classical embossing processes. A polymer layer is patterned by mechanical embossing using a stamp. However, in NIL this patterned polymer layer later serves as an etching or lift-off mask analogously to photolithographic processes. The term nanoimprint lithography was first introduced by Chou et al. in 1995 [132]. In that work, thin thermoplastic resist materials were imprinted in a very similar way to hot embossing processes. This process is widely known as NIL; however, it has often also been named hot embossing lithography (HEL) [133] or thermal NIL [134]. In 1996, Haisma et al. were the first to introduce a UV-curing NIL process later on known as UV-NIL [135]. This technology evolved and was especially pushed forward due to it being considered an alternative technology for future generation integrated circuit applications [6]. Reviews for different types of NIL can be found in References $[133,136,137]$. In the following, two tools for NIL developed within 
this work to meet specific demands for solar cell texturisation will be explained. The principles of these tools for NIL are sketched in Figure 4.4.

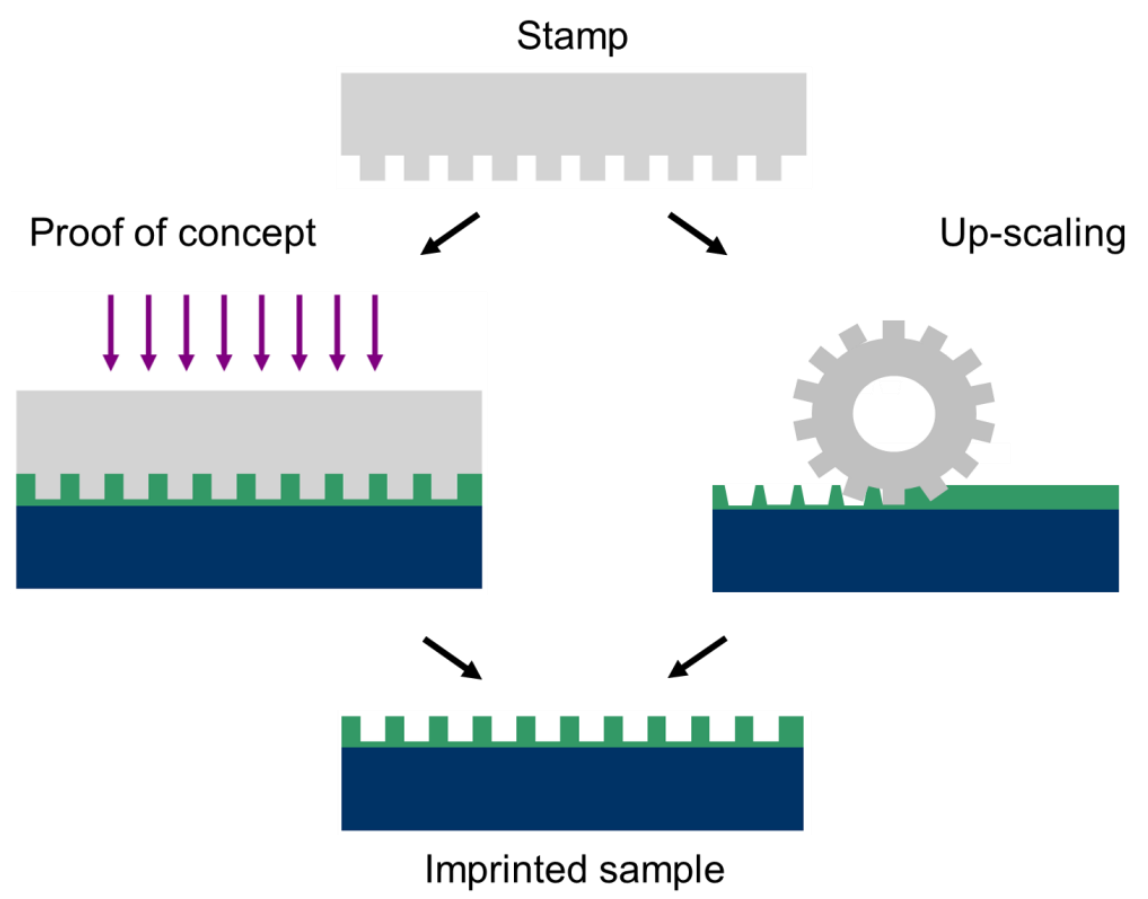

Figure 4.4: Sketch of the two types of nanoimprint lithography developed within this work. For the Roller-NIL patterning of an etching mask, a tapered shape of the resist is indicated. This effect can occur when trying to replicate a binary profile in a Roller-NIL process. The dimensions of the stamp's pattern and the diameter of the roller are not to scale.

In Figure 4.4, it is schematically shown that, when trying to replicate a binary pattern in a Roller-NIL process, a tapered profile shape in the resist mask can result due to the moulding and demoulding steps in Roller-NIL. This effect was not investigated and was also not observed to be crucial for the Roller-NIL tool developed as well as for the structures examined within this work. One reason for this behaviour is that the pattern dimensions are very small compared to the roller diameter.

The quality of an etching mask patterned via NIL strongly depends on the homogeneity of the so-called residual layer thickness, which is visualised schematically in Figure 4.5. This layer has to be as thin as possible to allow an immediate start of the etching process in the subsequent step. It also has to be as homogeneous as possible to obtain a uniformly textured surface later on. Two other characteristics of imprinted patterns are defined in Figure 4.5. These are namely the period (as we are dealing solely with periodic features within this work) and the pattern depth. The last two parameters are especially important for the 
use of soft stamp materials, because they can be used to quantify deformations in the stamp during the imprint process.

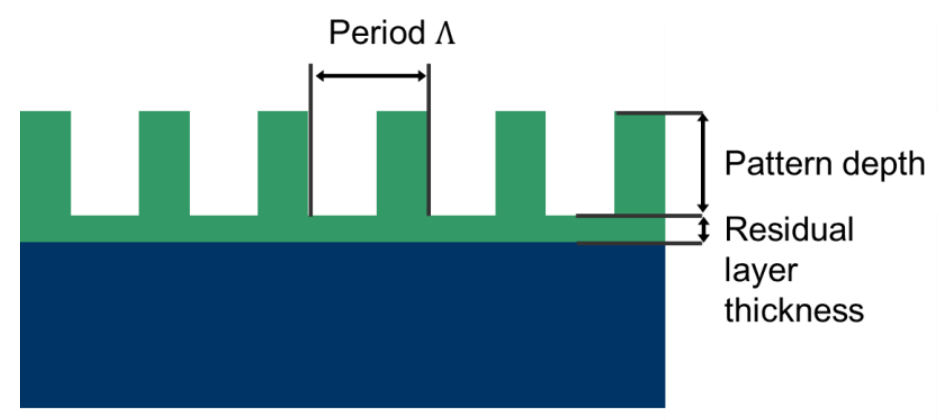

Figure 4.5: Schematic illustration of an imprinted pattern on a substrate. Some important characteristics to assess the quality of imprinted patterns are sketched: residual layer thickness, period and pattern depth.

\subsubsection{Development of a conventional UV-NIL tool}

The task of developing a conventional NIL tool had to be fulfilled meeting the following demands:

- uniform pressure distribution (potentially on rough substrates)

- minimal wedge errors for the embossing process

- a patterned area of $100 \times 100 \mathrm{~mm}^{2}$

- UV-curing of a negative resist (for opaque silicon substrates)

- high UV-transparency of components through which the exposure is realised

In this tool, a stamp shall be pressed into a low viscous resist and the exposure and thus the curing of the resist has to be realised through the stamp. Before this NIL process, a resist layer is spin-coated on the silicon substrate. The demoulding after the NIL process can be realised manually.

In Figure 4.6, SEM micrographs of two imprinted resist layers are shown. The thicker residual layer on the left hand side is a consequence of an unoptimised initial resist thickness after the spin coating. On the right hand side an optimised resist layer was applied leading to a very low residual layer thickness.

The homogeneity of the residual layer thickness depends on the homogeneity of the prevailing pressure distribution beneath the stamp. There are different approaches to build up the necessary pressure on the stamp. Classical hot embossing tools typically rely on rigid embossing plates onto which a force can be exerted, for example hydraulically. A lot of effort then has to be put into 
guaranteeing minimal wedge errors. Otherwise, these wedge errors lead to inhomogeneous pressure distributions and in the worst case pressure peaks on very small areas. To avoid problems like this, we aimed for a setup where the force onto the stamp is realised via an air cushion. This solution of applying air-pressure for very uniform pressure distributions is also used in commercially available imprint tools e.g. by Nanonex ${ }^{\mathrm{TM}}$ [138] or Obducat ${ }^{\mathrm{TM}}$ with their patented SoftPress ${ }^{\circledR}$ technology. The topic of pressure homogenisation is further tackled by the use of soft stamp materials like PDMS. There, as mentioned above, besides the Young's modulus the thickness of the stamp also plays a major role.
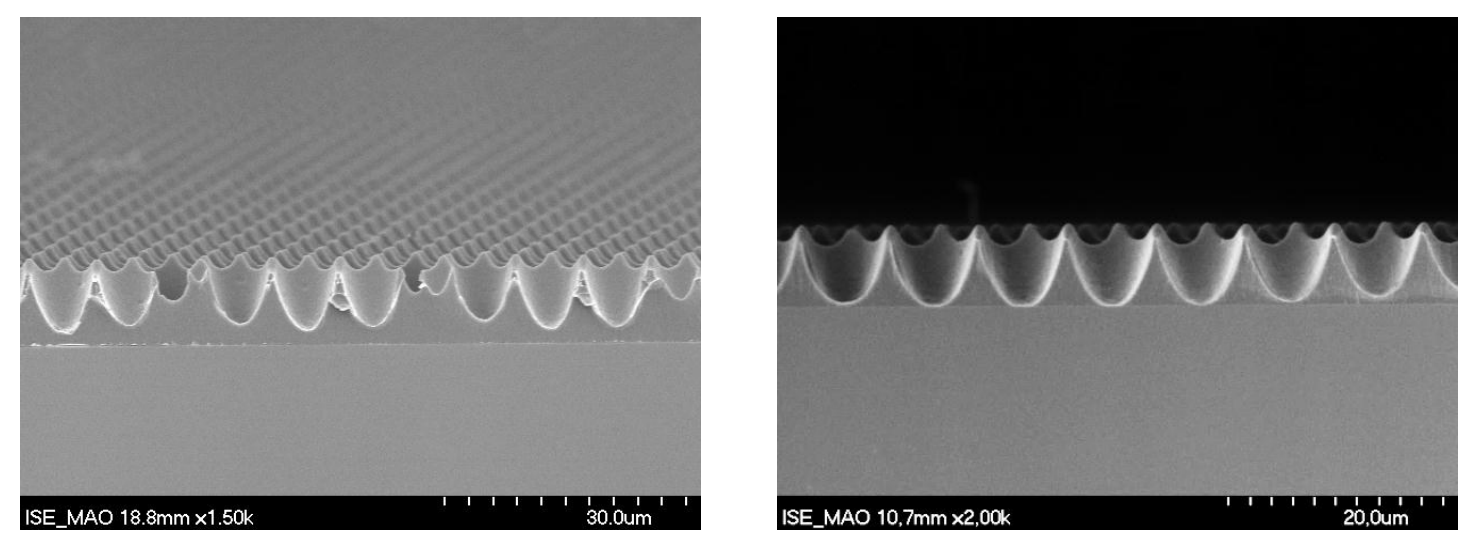

Figure 4.6: SEM micrographs of imprinted resist layers on top of glass substrates. For the sample on the left hand side, the residual layer thickness below the imprinted pattern is about $1 \mu \mathrm{m}$ and on the right hand side it is below $100 \mathrm{~nm}$. A very low residual layer thickness is essential for the subsequent etching process.

UV-curing resists are typically highly sensitive in the spectral range between 350 and $400 \mathrm{~nm}[139,140]$. Very often it is recommended to expose photoresists using i-line light sources $(365 \mathrm{~nm})$. To be able to easily switch exposure light sources, the tool is designed to permit the use of external light sources. As mentioned before, silicon is not transparent in this range and therefore the exposure has to be conducted through the stamp. Indeed all components through which the exposure is accomplished have to be transparent in the relevant wavelength range. The resulting setup is sketched in Figure 4.7. The NIL tool consists of an aluminium frame in which the stamp (bonded to a carrier quartz substrate) is mounted on springs. Below this stamp the wafer (already coated with photoresist) is inserted. Above the stamp, there is an air cushion made of UVtransparent $\mathrm{ETFE}^{10}$ foil. On the top, the aluminium frame is closed by a transparent cover, in which a pressure inlet is made. This whole setup can be placed beneath a UV-light source to conduct the NIL process.

10 ETFE: Ethylene tetrafluoroethylene is a UV transparent polymer with excellent mechanical properties in terms of tensile strength and elasticity. 


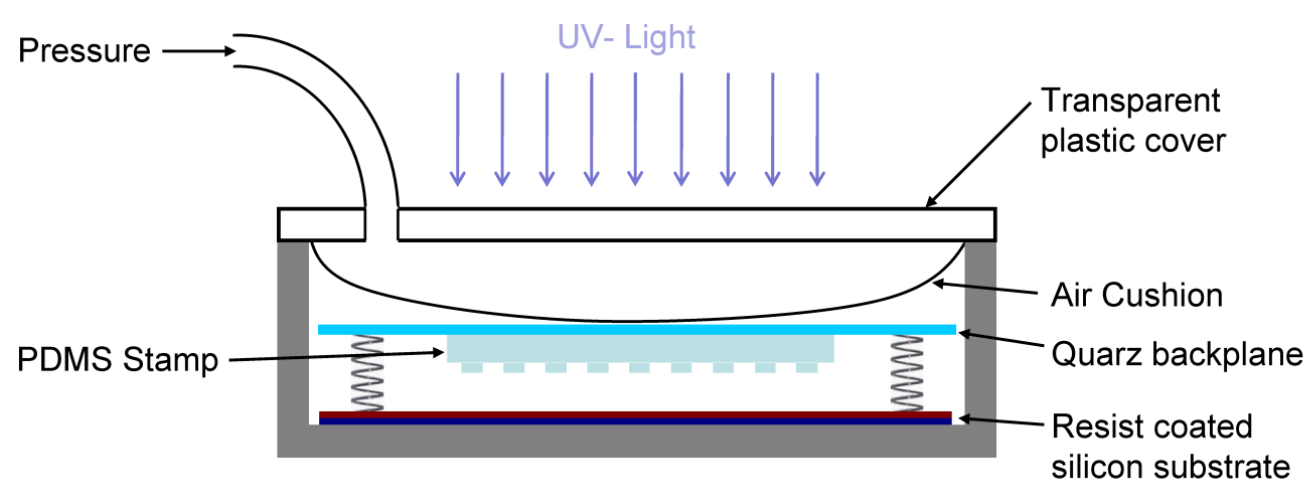

Figure 4.7: Sketch of the nanoimprint lithography tool relying on a planar stamp setup developed within this work. A similar concept was published in [138].

\subsubsection{Stamp fabrication}

Having originated a master structure via interference lithography and subsequent electroforming processes, we now want to replicate this master in order to fabricate a stamp for the nanoimprint lithography process. Reviews of possible techniques for stamp materials and fabrication can be found in [133, 136, 141].

In the following, some material requirements for the stamp will be listed. Afterwards these are explained for the type of nanoimprint lithography process we are aiming for:

- large area on potentially rough or wavy substrates (full wafer scale)

$\rightarrow$ flexible stamp materials

- fast processing in nanoimprint lithography

$\rightarrow$ UV-curing preferred to thermoplastic resist systems

$\rightarrow$ UV-transparent stamp materials therefore required (for opaque silicon substrates)

- easy maintenance

$\rightarrow$ preferably intrinsic anti-sticking properties

$\rightarrow$ cleanable

- up-scalability

$\rightarrow$ potentially useable for a Roller-nanoimprint lithography tool

When considering process development for the PV market, one always has to be aware of the necessity for large area processing combined with affordable costs. Thus, one cannot aim for step-and-repeat nanoimprint lithography based on the use of hard stamps. This sequential patterning process using small stamps allows a pattern transfer down to the sub-100 $\mathrm{nm}$ scale and therefore is interesting for 
microelectronics applications [142]. Full wafer scale nanoimprint processes require flexible stamp materials even for the very small waviness of monocrystalline silicon material [123]. This dramatically gains in importance when it comes to patterning etching masks on rough multicrystalline substrates as used for solar cell processing. However, when dealing with stamps made of materials of a low modulus of elasticity, other problems occur e.g. due to deformation effects. A trade-off therefore has to be found for the stamp's stiffness to achieve sufficient pattern fidelity as well as adaptability to rough surfaces. This also includes geometric considerations, because an important parameter for deformation as well as adaptability of the stamp is its thickness [143].

The thicker the stamp is, the more tolerant it is to rough surfaces, because more deformation in the PDMS bulk is allowed. However, for elastomeric materials like PDMS, the Poisson ratio is typically very close to 0.5 [144]. That means the volume stays nearly constant during deformation processes. Thus, a thicker PDMS stamp leads to a higher lateral deformation. Conversely, using a very thin PDMS stamp, the sideways deformation introduced by the constancy of volume can be minimised, however the tolerance to adapt to rough or wavy substrates is also minimised.

The effect of the lateral deformation is visualised in a simplified finite element method (FEM) simulation using the software COMSOL. In the simulation, it is assumed that the PDMS stamp is bonded to a glass substrate and thus, at the PDMS-glass interface, no in-plane movement of the PDMS is allowed. The stamp is pressed onto a flat and fixed surface with the pressure being applied from the back of the glass substrate. At this interface, the stamp is normally structured; however, in this simplification, the stamp's surface is being treated as flat. Furthermore, any friction effects at this interface are neglected. In reality, the structured stamp will be pressed into a low viscous resist layer in the case of the UV-NIL. This is a simple model, but it helps to visualise the dependency of the stamp's thickness on the lateral movement at the resist interface. This lateral movement can introduce a distortion of the replicated pattern. In Figure 4.8 a) an exemplary simulation result showing the examined geometry is shown. The simulation has been carried out for a range of stamp thicknesses. The displacement in the $x$-direction for different stamp thicknesses is shown in Figure $4.8 \mathrm{~b})$. 

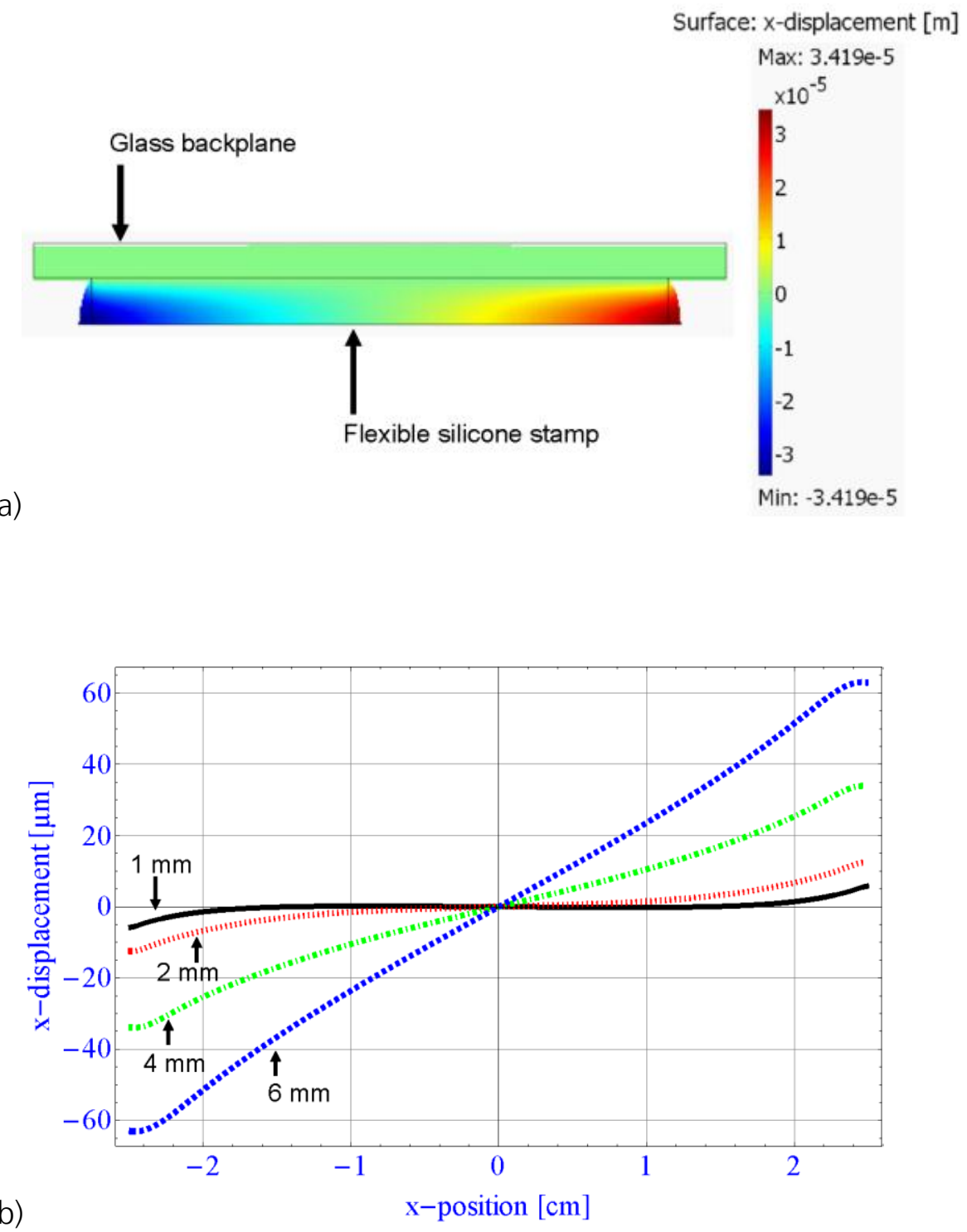

Figure 4.8: In a) the result of a FEM simulation of the stamp deformation is shown. The silicone stamp is bonded to a glass substrate. The stamp thickness in this case is $4 \mathrm{~mm}$ and its size is $5 \times 5 \mathrm{~cm}^{2}$. The material properties used for the PDMS in this simulation are a modulus of elasticity of $2 \mathrm{MPa}$ and a Poisson ratio of 0,49. The applied pressure is 0,2bar. The deformation in the graphic is scaled to better visualise the effect. b) shows the local lateral deformation at the lower surface of the stamp for thicknesses of $1 \mathrm{~mm}$ (continuous), $2 \mathrm{~mm}$ (pointed) $4 \mathrm{~mm}$ (dotdashed) and $6 \mathrm{~mm}$ (dashed).

Figure 4.9 shows results of the NIL process using a thin stamp on a rough surface (a) and a thick stamp on a smooth surface (b). For the thin stamp, the pattern is replicated very well, but the roughness of the wafer is not compensated. For the thick stamp, an outer region of the stamp's replication is shown in the SEM micrograph visualizing the effect of lateral deformation on the replicated pattern. 
a)
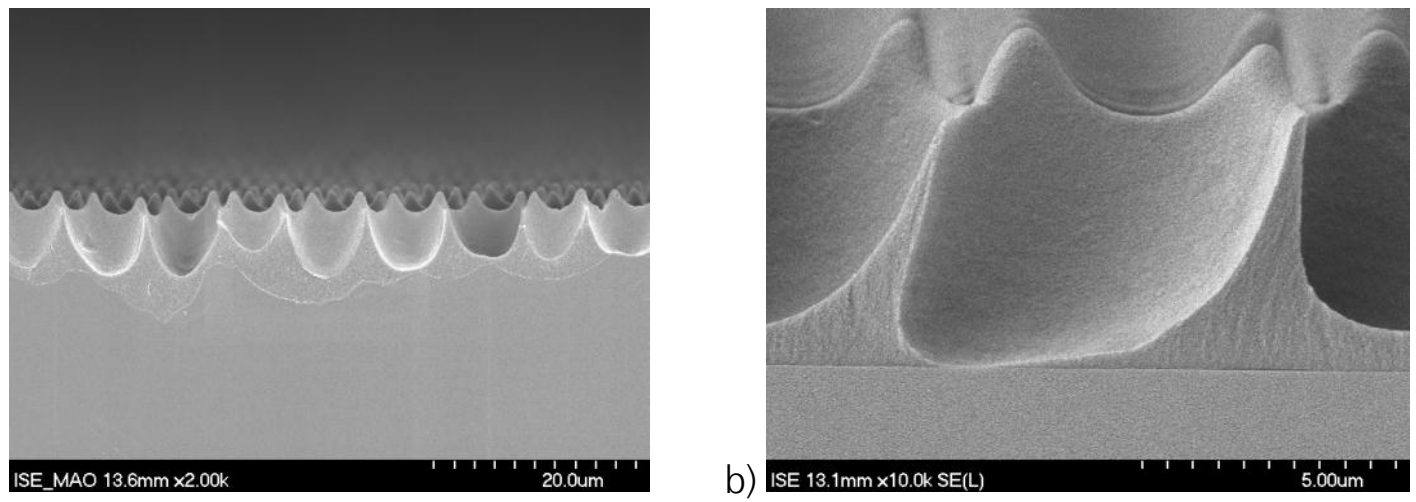

Figure 4.9: SEM micrographs showing results of imprinted polymer layers using different stamp thicknesses. In a), a stamp with a thickness of $2 \mathrm{~mm}$ was used to imprint on top of a rough surface. The stamp is not capable of compensating the surfaces roughness. In b), a $6 \mathrm{~mm}$ thick stamp is used to imprint onto a smooth glass surface showing a stamp deformation in an outer region. Looking at this cross section, this would be a region on the right side of the stamp, given that the deformation is in this direction.

For decreasing pattern dimensions, e.g. when realising diffractive elements in the sub-micrometre scale, other problems arise due to the small Young's modulus of standard PDMS, leading to a pattern collapse [145]. This problem can either be overcome by increasing the Young's modulus as described in [146] or by using other polymeric stamp materials like for example ETFE as published in [136, 147]. One very interesting approach is based on the fabrication of stacked PDMS stamps of layers with different Young's moduli [148]. This allows the combination of both the adaptability of soft stamps and the pattern fidelity of hard materials and is described in more detail in section 5.2.

PDMS as well as ETFE possess excellent intrinsic non-sticking behaviour as a result of a low surface free energy, so that an anti-sticking coating does not necessarily have to be applied [149]. Anti-sticking coatings are mainly based on fluorine chemistry and the major drawback of such coatings concerns their durability [136, 150]. A comparison of fabricating stamps with sub-micrometre features using PDMS and ETFE will be given in subsection 5.2.2.

UV-curing processes are supposed to allow shorter process times than thermoforming processes. Patterning a thermoplastic material requires exposure to temperature cycles [83]. This presents particular difficulties when aiming for a roller-imprinting process as we do, although it has been reported to work successfully [151]. Nevertheless, we aim for UV-curing processes, since they are more promising for high throughput processes [152]. In UV-curing NIL, the minimal process time is determined by the filling of the stamp's cavities, which can be quick due to very low viscous resists, and the necessary exposure dose to guarantee a sufficient cross-linking of the resist. Both PDMS as well as ETFE show a 
transmission of about $90 \%$ in the UV range around $365 \mathrm{~nm}$ for reasonable stamp thicknesses, and since both materials are flexible, it is also possible to wrap them around a roller.

\subsubsection{Resist investigations for NIL}

In the following, investigations of different types of resists for NIL are described. These investigations were conducted using the conventional NIL tool and shall help to set up processes for the Roller-NIL process later on. Regarding the type of NIL we are aiming for as well as the application of solar cell texturisation, the following demands have to be met by the resist:

- negative tone UV-curing resist system

- moderate necessary exposure dose

- low viscosity to allow short filling times of the stamps cavities

- compatibility with stamp (no inhibition of cross linking, no sticking to the stamp)

- sufficient adhesion to silicon (preferably without adhesion promoter)

- etch resistivity (in fluorine plasma or potentially also in acidic wet-chemical solutions)

- no contaminations for delicate solar cell processing (metal contaminations)

- clean removability after etching processes (lift off, dissolving, ashing)

- low price

Some of these points are essential (e.g. UV-curing, no contaminations), some others might contain contrary requirements (e.g. non-sticking vs. adhesion, etch resistivity vs. removability). However, all points have to be fulfilled sufficiently to pass through the complete process chain successfully.

Negative tone photoresists are typically based on monomers or pre-polymers, a photoinitiator and optionally organic solvents. The viscosity of the resist can be adjusted either by the length of the pre-polymer's molecular chains or by diluting the resist with a solvent. The influence of the small amount of photoinitiator on the viscosity is negligible. There are mainly two classes of polymers, which differ in their type of polymerisation: free-radical polymerisation (acrylates) and cationic polymerisation (epoxy systems). Both of these systems reveal interesting properties and thus both were considered for this application. Acrylate systems potentially need lower exposure doses and the polymerisation reaction proceeds very fast as a result of the presence of highly reactive radicals and monomers [153]. Cationic 
polymerising systems do not suffer from oxygen inhibition, show potentially less shrinkage and exhibit dark polymerisation after an exposure is stopped [154]. One cationic system investigated during this work belongs to the SU8 product family developed by IBM [155], which is commercially available from MicroChem. Freeradical polymerising systems used for the presented investigations are of the Laromer product family from BASF and mr-UVCur06 as well as mr-UVCur21SF from Micro Resist Technology. The prices of the resists listed above cover a wide range. This of course will be crucial when it comes to a transfer of the investigated process chain into industrial environments. However, in this early stage of development no resists were excluded on the basis of too high prices.

For all these systems, the compatibility with stamp materials is given. Some resists require adhesion promoters to avoid a sticking to the stamp's surface (e.g. SU8 and mr-UVCur06). However, these adhesion promoters also allow a removal via lift-off after the etching process and are therefore not necessarily to be considered as undesirable additional process steps. The removal of negative tone resists is in general not trivial. Due to the highly cross linked polymers, dissolving with organic solvents is typically not possible (especially after plasma etching processes with harsh radiation and additional thermal energy leading to a hard bake). If the resist is purely polymeric with no inorganic additives, oxygen plasma treatment can be used to burn the resist forming carbon monoxide, carbon dioxide, water and other volatile gases [156]. Several publications report on increased etching rates for the plasma ashing, when adding $\mathrm{CF}_{4}$ to the oxygen [157-160]. Other methods for resist removal are burning, swelling in organic solvents (leading to a lift-off), oxidation in molten salt baths [157] and, as is becoming more and more popular in semiconductor industry, the utilisation of ozonised water to remove organic resist residues [161].

\section{Considerations regarding soft PDMS stamps \& solvent assisted imprint processes}

As mentioned above, the viscosity of resists is often adjusted using organic solvents. When using solvent based resists in NIL, some problems might arise. Depending on the vapour pressure of the solvent and the environmental process conditions during the imprint, some evaporation can occur. This leads to a gaseous solvent accumulation between stamp and resist. For a non-permeable stamp material, this is critical, since then there might occur an inhibition of cross-linking processes in the resist material due to local solvent accumulations, leading to problems in terms of pattern fidelity and homogeneity. This problem can be solved by using PDMS as the stamp material. PDMS allows the gaseous solvent to migrate through it and thus the stamp is no barrier for the outgassing of solvent [133]. 
However, this useful property can also be interpreted as a negative characteristic, since this also leads to a moisture expansion or swelling. This again might lead to a pattern distortion and might also set limitations to the lifetime of the stamp. In the present study, these negative effects did not play a major role and the positive effect of the permeability outweighed them completely for solvent based resists.

The viscosity of the resist changes dynamically due to the solvent evaporation. This leads to a varying flow behaviour of the resist as a result of the decreasing solvent content over time. This is especially crucial when imprinting with stamps of a low Young's modulus. As a result of the softness of the stamp material, a deformation can occur. The consequence of this physical process on the result of the NIL process is visualised in Figure 4.10. There, a solvent based resist was applied on a glass substrate with a parameter variation of the spinning velocity. All other relevant parameters, such as spinning duration, imprinting pressure, time before imprint and exposure duration, were kept constant and are summarised in Table 4.2. The resist serving as an example for solvent based negative tone resists in this section is SU8-2002 ${ }^{\mathrm{TM}}$.

Table 4.2: Process parameters for NIL studying the solvent dependency on the resulting patterns.

\begin{tabular}{|c|c|}
\hline Process Step & Parameters \\
\hline Prebake of the substrates & 2 min @ 180 $\mathrm{C}$ (Hotplate) \\
\hline Spinning time & $15 \mathrm{~s}$ \\
\hline Spinning velocity & $300-1000$ rpm \\
\hline Imprint pressure & $0.2 \mathrm{bar}$ \\
\hline Holding time before exposure & $1 \mathrm{~min}$ \\
\hline Exposure time & $\begin{array}{l}\text { 1:30 min (resulting Dose }{ }^{11} @ 365 \mathrm{~nm}: 100 \\
\mathrm{~mJ} / \mathrm{cm}^{2} \text { ) }\end{array}$ \\
\hline Stamp size & $50 \times 50 \mathrm{~mm}^{2}$ \\
\hline
\end{tabular}

${ }^{11}$ The light source is a broad band Xenon lamp. The intensity was measured using a commercially available UV-meter (Lutron UVA-365). This UV-meter has a narrow Gaussian shaped spectral sensitivity with a maximum at about $365 \mathrm{~nm}+-20 \mathrm{~nm}(1 \sigma)$ according to the datasheet. 
a)

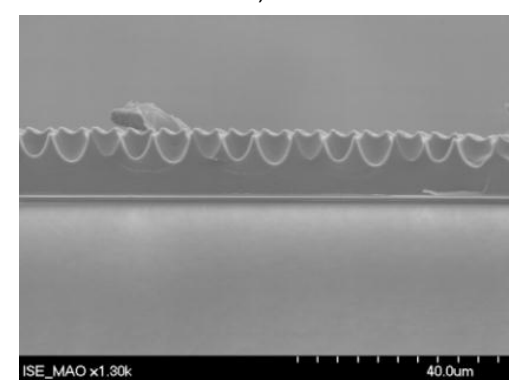

b)

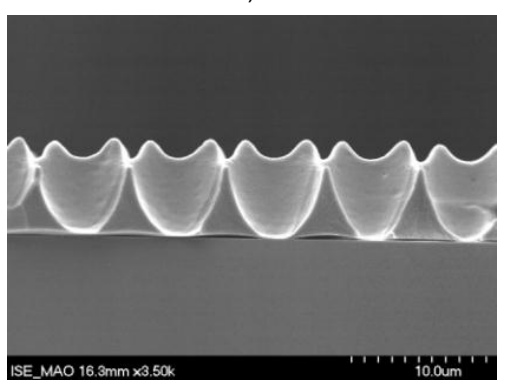

c)

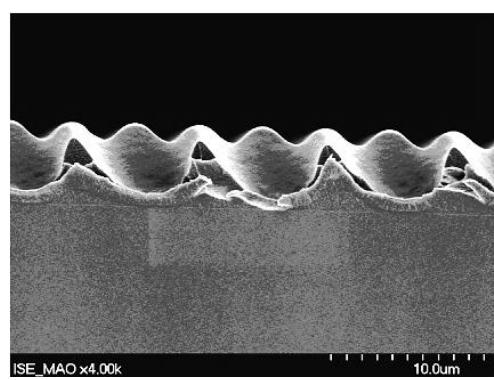

Figure 4.10: SEM micrographs of imprinted patterns in a solvent based negative resist on glass substrates. The resist was applied via spin coating with spinning velocities of $300 \mathrm{rpm}$ (a), $500 \mathrm{rpm}$ (b) and $1000 \mathrm{rpm}$ (c).

Figure 4.10 shows imprints with (a) a too thick residual layer, (b) a good result in terms of the pattern quality and residual layer thickness and (c) a deformed pattern. In (a), for a low spinning velocity the resulting residual layer is too thick, since the initial thickness after the spin coating process is too high. Then, for increasing spinning velocities in (b), the pattern is replicated nearly in its full structure depth and a near zero residual layer thickness (below $100 \mathrm{~nm}$ ) is achieved. For higher spinning velocities, one would expect that the depth of the replicated pattern is decreasing while the residual layer thickness stays near zero. However, for higher spinning velocities, more solvent evaporates, so that the resist shows a higher viscosity and the soft stamp is more likely to be deformed in the imprint process. In (c), although the initial resist thickness is less than in (b), the residual layer is again above $200 \mathrm{~nm}$.

SU8-2002 was applied to glass substrates at different spinning velocities and a number of characteristics of the coated layer and subsequently imprinted patterns were measured. The results are shown in Figure 4.11. The solvent content in the resist layer was determined gravimetrically after the spin coating process (Figure 4.11 (a) right scale). The resist weight was measured directly after the coating process as well as after driving out the solvent via baking $\left(15\right.$ minutes at $90{ }^{\circ} \mathrm{C}$ on a hotplate, Figure 4.11 (a) left scale). The solvent used in this resist is Cyclopentanone and its initial concentration in the resist is $70 \%$ by weight according to the datasheet [162]. The resulting imprint quality was evaluated by measuring the initial resist thickness, the pattern fidelity (detected by the structure depth) as well as the residual layer thickness (Figure 4.11 (b)). As expected, for the initial resist layer thickness an exponential decay for increasing spinning velocities can be seen. The residual layer thickness first decreases, then, after reaching a minimum, it increases again. The structure depth at first increases surprisingly. This might be explained by the fact that in this regime the resist is very thick and has a very low viscosity, leading to a kind of swimming or smearing of the stamp by 
which large deformations are induced. These deformations reduce the resulting pattern depth. At around $500 \mathrm{rpm}$, a maximum is reached, where the full pattern depth is replicated. For further increasing spinning velocities, the pattern depth decreases again, due to both too low an initial resist thickness and a decreasing solvent content in the resist.

a)

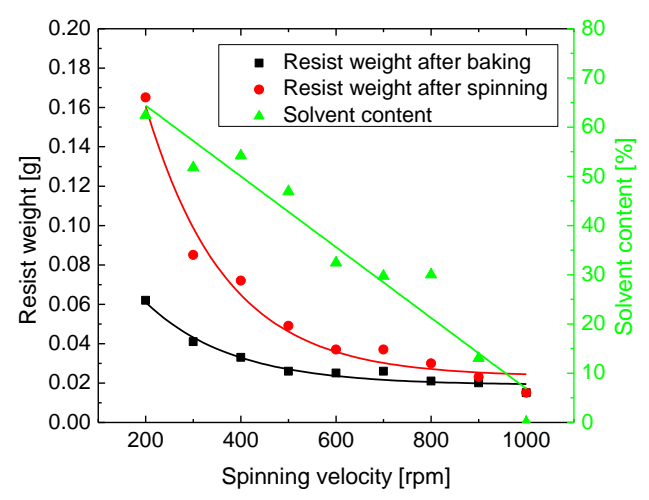

b)

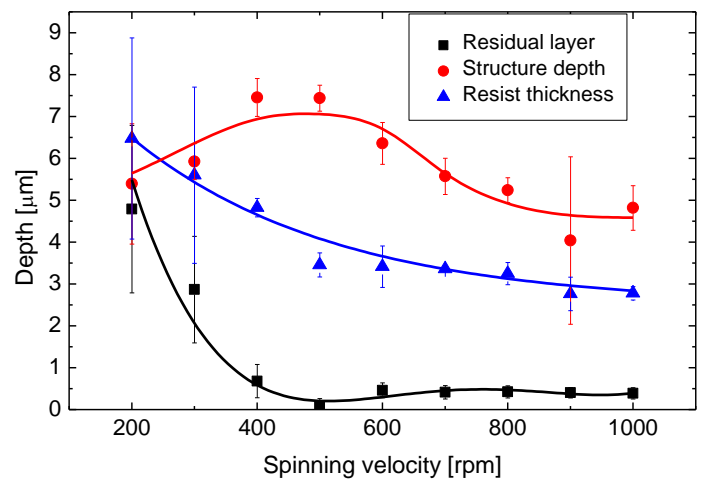

Figure 4.11: a) shows the resist weight wet and after baking out the solvent and the solvent content for different spinning velocities during the spin coating process for constant spinning durations. The weight relates to the substrate area of $75 \times 75 \mathrm{~mm}^{2}$. The measurement uncertainty for the applied scale is $\pm 5 \mathrm{mg}$. b) shows the initial resist thickness, residual layer thickness and replicated structure depth after a NIL process for the corresponding spinning velocities. Measurements were done by SEM analysis. Lines are guides to the eyes.

Using the optimum spinning velocity of $500 \mathrm{rpm}$, a reproducible and satisfactory NIL process could be set up. This set of parameters was used for proof-of-concept studies using the conventional NIL tool also to evaluate the following process steps from etching as far as solar cell fabrication. However, as a conclusion of these investigations, it can be stated that there is a complex relation between process and resist parameters for NIL, when using soft PDMS-like stamp materials and solvent based resists applied by spin coating. By additionally taking the initial solvent concentration and spinning duration into consideration, this becomes even more complicated. Looking at all these parameters, it becomes clear that the maximum of replicated structure depth and the minimum of the residual layer thickness in Figure 4.11 b) are achieved for identical spinning velocities. Therefore, the developed process can be understood as a solvent assisted NIL process. There are different types of solvent assisted NIL processes, where a dry polymer film is liquefied using an organic solvent atmosphere to allow an imprint (e.g. by Voicu et al. [163]). What both the processes investigated within this work, as well as the one reported by Voicu have in common is that a certain solvent content has to be realised within the resist layer. While we try to maintain a certain solvent content, the other approach relies on introducing it. A mind map showing input parameters 
for a successful solvent assisted NIL process under the described boundary conditions (PDMS stamp, spin-coated solvent based resist) is shown in Figure 4.12.

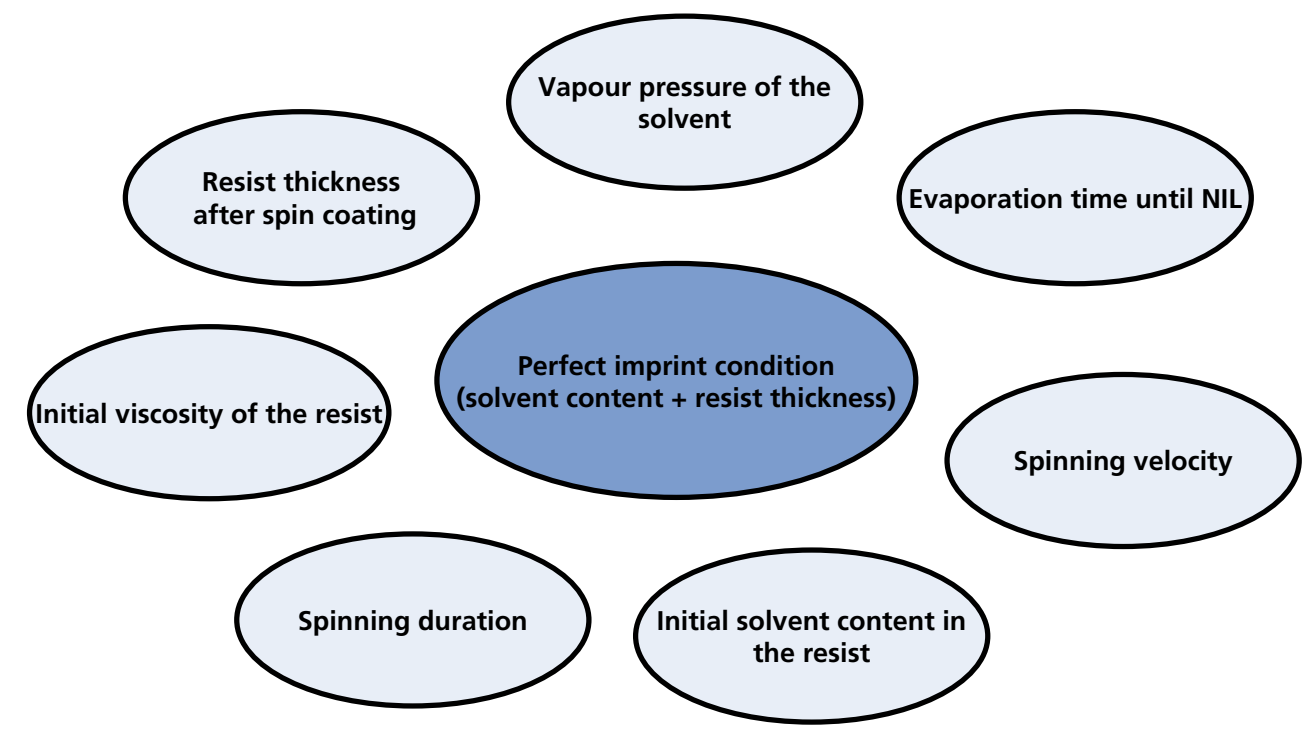

Figure 4.12: Sketch of the input parameters for the solvent assisted NIL process using PDMS stamps and solvent based resists applied via spin coating. There are interactions between almost every pair of processes or material characteristics listed in this drawing. Thus, a successful imprint necessitates the consideration of all these parameters.

\section{Implications for the applicability of this solvent-assisted process for Roller- NIL}

By the parameters "spinning duration" and "evaporation time until NIL" in Figure 4.12, time is captured as a relevant process parameter. In the following, the investigation into how crucial this time-dependent solvent evaporation process is on the quality of replicated patterns, is further on conducted using SU8-2002 $2^{\mathrm{TM}}$ as the resist material. This time dependency might be critical for Roller-NIL processing, since a sequential imprinting is conducted along the substrate. A gradual change of the viscosity during the imprint of one substrate might lead to a variation of the pattern fidelity. Of course this scenario only holds for application by spin coating, which is what was applied within this work. However, the findings of the present investigation are more generally important for a deeper understanding of imprinting processes with soft stamps and solvent based resist materials.

The time dependency can be evaluated by picking out the optimum spin coating and NIL parameters from Figure $4.10 \mathrm{~b}$ ) and Figure $4.11 \mathrm{~b}$ ), and inserting a defined evaporation time between the spin coating and the NIL process. Then, the influence of the evaporation process on the imprint quality can be isolated. By doing this, one would expect that, starting with an identical initial resist thickness after spin coating, the viscosity increases over time and, for constant imprint 
conditions, the stamp then gradually becomes unable to penetrate the resist layer completely. Deformation in the soft stamp material might also occur, which further reduces the resulting structure depth. Figure 4.13 shows the results of imprint quality for varying evaporation times and confirms the thoughts just mentioned before. In this case, the imprint quality was assessed by the structure depth as well as the residual layer thickness. It is visible that the quality of the imprinted pattern is reduced for very short waiting times. This decay in replication quality then continuously proceeds.

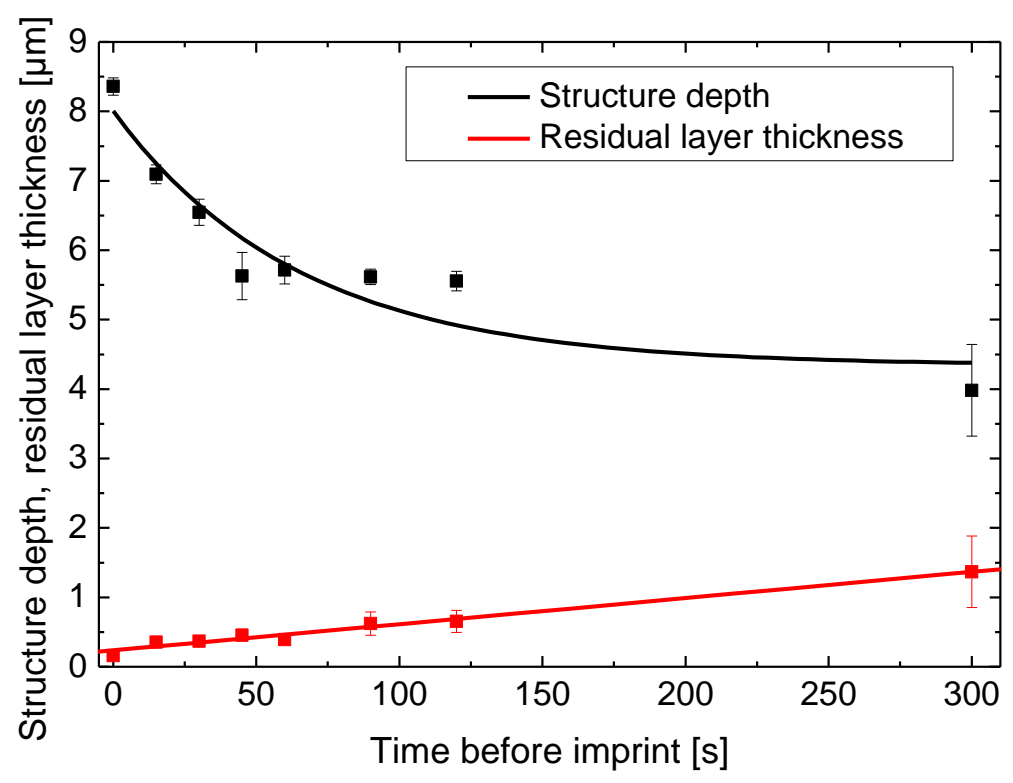

Figure 4.13: A graph showing the resulting structure depth as well as the residual layer thickness over a varied time between the spin coating and the NIL process. The structure depth and residual layer thickness were measured using a SEM. Lines are guides to the eyes.

Assuming this decay in replication quality is related to a solvent evaporation from the resist, leaving it more highly viscous, we want to investigate the dynamics of the solvent outgassing. This is done by evaluating time-dependent spectral reflection measurements on spin-coated resist layers on top of glass substrates. For these measurements, an Optical Multichannel Analyser (OMA) was applied. The optical interfaces that are relevant for this reflection measurement are sketched in Figure 4.14. 


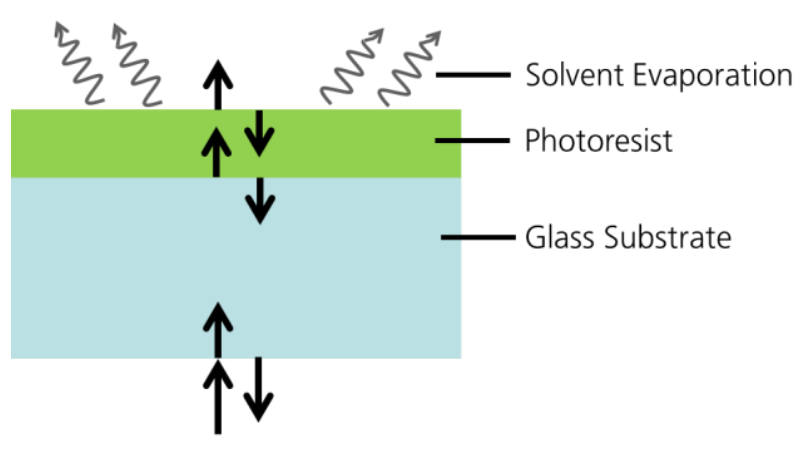

Figure 4.14: Sketch of the measurement setup to determine the decay in solvent content within the resist layer. In discrete time steps, spectral reflection measurements of a resist coated glass substrate are conducted. The illumination of the sample as well as the detection of reflectivity is both conducted from the back side.

In Figure 4.15, spectral reflection measurements are shown exemplarily directly after the spin coating as well as after 30 seconds and 200 seconds. The curves of these measurements give information about the refractive index and thickness of the resist layer. Since the resist layer acts as an optically thin layer, interference effects occur, which lead to a modulation of the reflection amplitude over the wavelength. This modulation can be used to calculate the refractive index of the resist at the moment of each measurement. This is important, since the refractive index of the polymeric resist material differs from the organic solvent ${ }^{12}$. The evaporation of the solvent, which has a lower refractive index than the filling material, leads to an increasing refractive index of the resist layer. This refractive index can be calculated either by using the amplitude of the reflection's modulation or the average over a whole period of this modulation. Both possibilities can then be used as an input to calculate the refractive index using the transfer-matrix formalism either treating the resist layer as optically thin or neglecting interference effects [53].

\footnotetext{
12 Dry baked SU8 - 2002 has a refractive index of about 1.61 (ellipsometric measurement). The solvent cyclopentanone has a refractive index of 1,437 according to [164]. Both values are given for a wavelength of about $590 \mathrm{~nm}$ (D-line).
} 


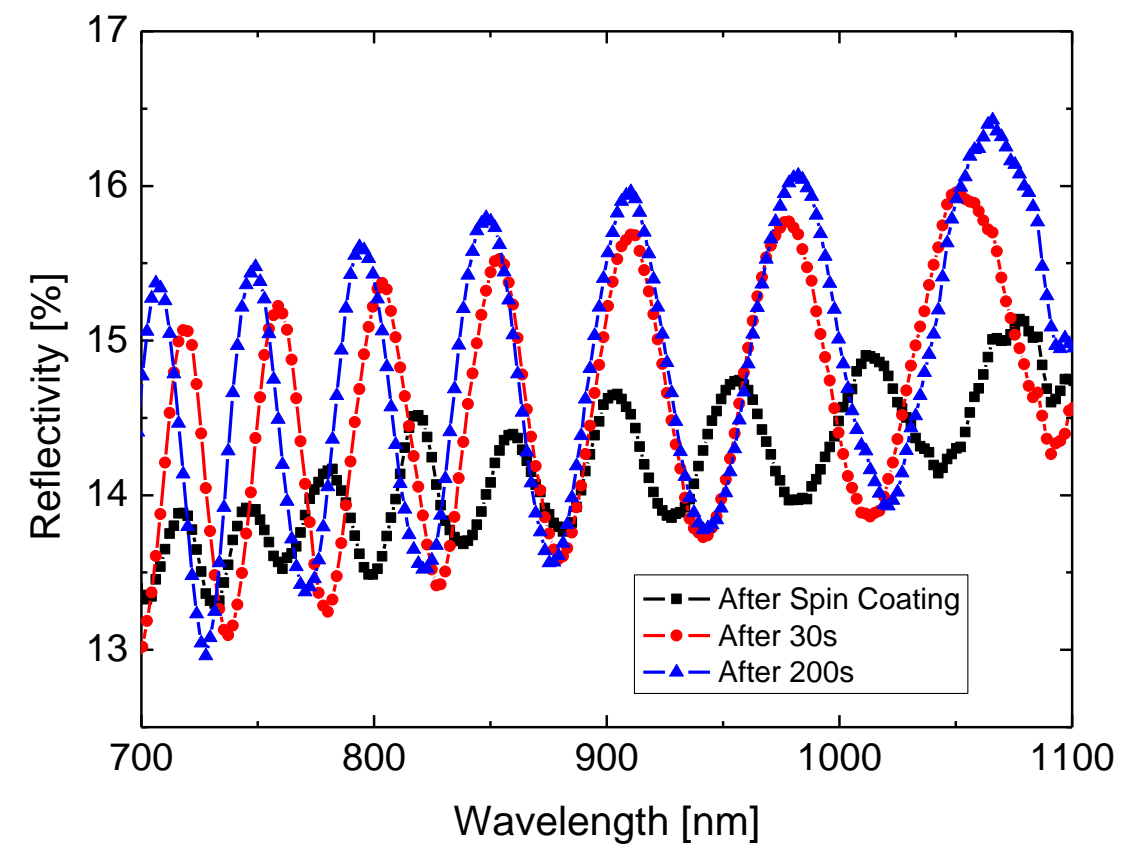

Figure 4.15: Spectroscopic reflection measurement of a resist coated glass substrate directly after spin coating, after $30 \mathrm{~s}$ and after $200 \mathrm{~s}$. The measurements were conducted using an Optical Multichannel Analyser (OMA). The amplitude of this swinging behaviour can be used to calculate the refractive index at the time of each measurement. Using this refractive index and the position of reflection peaks the thickness of the resist can also be calculated.

After the determination of the time-dependent refractive index, the reflection measurements, as shown in Figure 4.15, can be used to calculate the resist thickness for each reflection measurement. Neighbouring peak positions of the modulation of the reflection measurement (with the $\lambda_{i}$ being the wavelengths for which maxima are detected with the index $i$ for the numbering of maxima in a specified wavelength range) and the present refractive index $n$ can be used to extract the order $k$ of how many standing waves fit into the resist layer via:

$$
\frac{k \lambda_{i}}{n} \equiv \frac{(k+1) \lambda_{i+1}}{n}
$$

The solution of this equation for $k$ then can be used to calculate the resist thickness by

$$
\text { thickness }=\frac{k \lambda_{i}}{2 n}
$$

This can be evaluated for each peak in the considered wavelength regime to minimise measurement uncertainties. Several measurement sequences and the resulting thickness variations are plotted in Figure 4.16. Different measurement sequences were conducted to test the reproducibility, and since only a limited 
number of measurements could be done within one sequence, different time steps were applied (time steps between measurements from 2 to 10 seconds). For the calculation of these values, a constant averaged refractive index between $800 \mathrm{~nm}$ and $1000 \mathrm{~nm}$ was assumed for each time step.

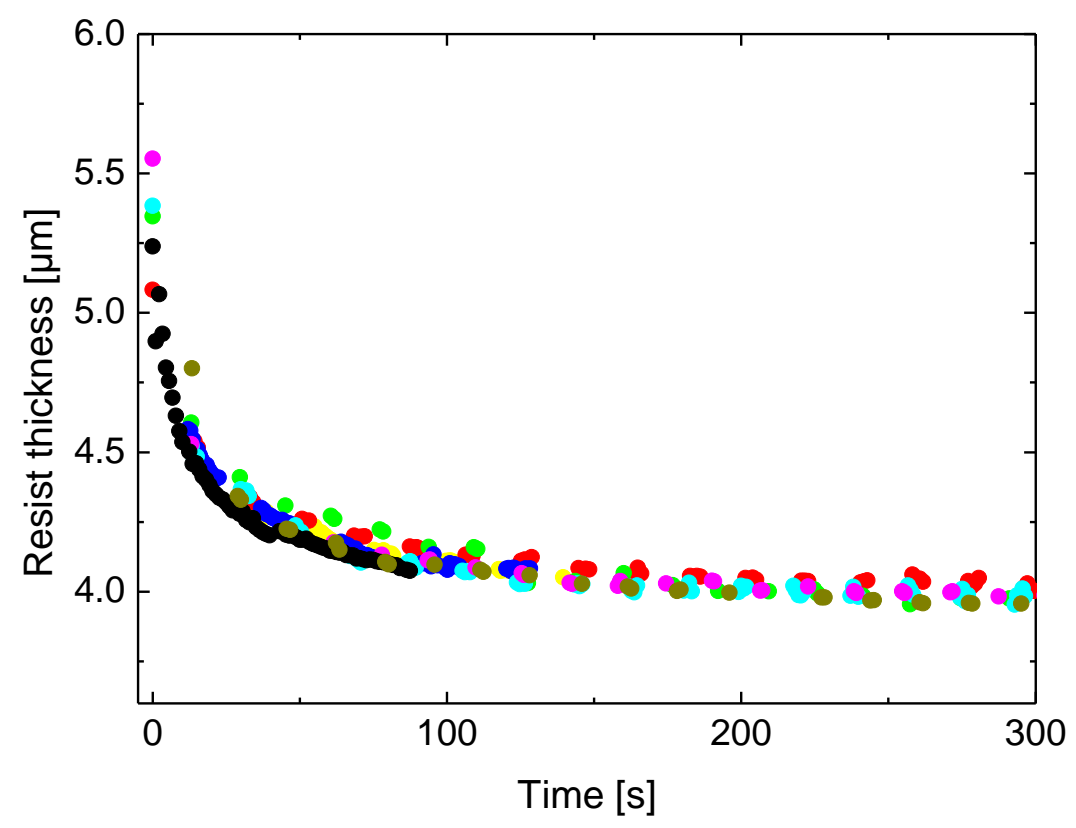

Figure 4.16: Calculated time-dependent resist thicknesses used to visualize the kinetics of the solvent evaporation. Input for these calculations are spectroscopic reflection measurements captured in a setup as sketched in Figure 4.14. The colours correspond to different measurement sequences to test the reproducibility.

The resulting decay in resist thickness visualises the evaporation of the solvent and, as can already be seen after a few seconds, the resist thickness and thus the solvent content is reduced drastically. The results of these measurements indicate that the solvent evaporation shows a strongly dynamic behaviour, especially in the very first seconds after the spin coating process, and that an exponential decay in the resist thickness can be assumed. This decrease in solvent content is related to an increase in viscosity and thus can be interpreted as one potential source for decreasing quality for the imprinting quality over the time. As mentioned before, this behaviour might be critical when it comes to a sequential imprinting process as it is to be realised in a Roller-NIL process. On the other hand, this investigation also shows some ways to circumvent this problem of an increased viscosity due to solvent outgassing. These options will be discussed in the following section related to the development of the Roller-NIL tool. 


\subsubsection{Development of a Roller-UV-NIL tool}

Roll-to-roll coating and patterning are well known and established technologies that are used for several printing applications. These applications cover fields from newspaper printing to the printing of electronic circuits. Roll-to-roll processing is a synonym for high-throughput mass production, often combined with the patterning of UV-curable resins. As the name roll-to-roll implies, this technology is restricted to the processing of flexible substrates, such as to pattern foils, to fulfil defined optical functions for example in display applications (e.g. collimating films, diffusers or polarisers). Looking at photovoltaics, this high-throughput technology might make the fabrication of organic solar cells very attractive [165]. For the patterning of resists on flexible substrates using roll-to-roll processes, it is possible to allow long contact times of the stamp and the substrate, since the coated substrate can be wrapped around the patterned stamp-roll. What's more, the resist coating of the substrates can easily be integrated into such setups as described in [166].

For the application of patterning an etching mask on wafer based silicon, one cannot realise a setup like this, since we have to deal with stiff and very brittle substrates. Therefore, the tool we have to develop could be better described by the expressions roll-to-sheet, roll-to-plate or Roller-NIL. The expression Roller-NIL using a thermal imprinting process was first introduced by $\mathrm{H}$. Tan et al. in 1998 [151]. Roller-NIL processes based on UV-curing of photoresists were later introduced based on different concepts in [167-171]. In [167], a resist layer is patterned on transparent glass substrates, so that an exposure through the substrate can be realised. In [168], a patterned foil is used as stamper. This foil is rolled onto the resist coated substrate, so that the pattern in the foil is filled. Afterwards, the foil remains on the substrate until a UV exposure through the foil is realised. After the curing of the resist, the foil is peeled off. In this case, the patterned foil is a disposable stamp. In [169], a similar approach is described where a flat stamper is sequentially rolled onto a coated substrate and the exposure is conducted afterwards through the stamper. In [170] and [171], approaches are described where the stamp is wrapped around a roll or a system of rolls and the light source is integrated within the roller system shining through the stamp.

For a stamp being wrapped around a single roll, a critical point results from the processing of stiff substrates: the contact area between the stamp and the resist coated substrate is drastically reduced (for a stiff stamp situated on a roll, this contact is in the limiting case just a line contact). Nonetheless, we aimed for this concept relying on a single roll instead of a system of multiple rolls on which the stamp is fixed, because of a reduced complexity. For this concept, in a short contact time the stamp's cavities have not only to be filled with the low viscous 
resist, but also a curing of the resist has to be realised. This curing does not necessarily mean that a complete cross-linking of the resist has to be assured, but rather that the resist has to be sufficiently hardened, such that, after a demoulding of the stamp, the pattern in the resist is stable enough not to reflow. A complete cross-linking of the resist could then be conducted separately after the Roller-NIL process. We tried to solve this problem by using flexible stamp materials, which can be deformed leading to an increased contact area when applying pressure. Furthermore, as described in chapter 2.3 we want to use flexible stamp materials anyway to allow an adaption of the stamp to rough surfaces of mc-Si wafers. Nonetheless, the contact area is comparatively small compared to classical roll-toroll processing.

Besides the issue of longer contact times, in the roll-to-roll processing the resist can be UV-cured in contact with the stamp by exposing through transparent foils, which can be used as substrate materials. Thus, non-transparent metal rolls can be used for the stamp. Since our substrate material (silicon) is not a UV-transparent material, we have to find another way to couple in the UV-light for the curing of the resist. This is realised by exposing through the stamp, setting even more restrictions to the material choice. Figure 4.17 shows schematically the setup of a standard roll-to-roll tool as well as the Roller-NIL tool developed within this work.

a)

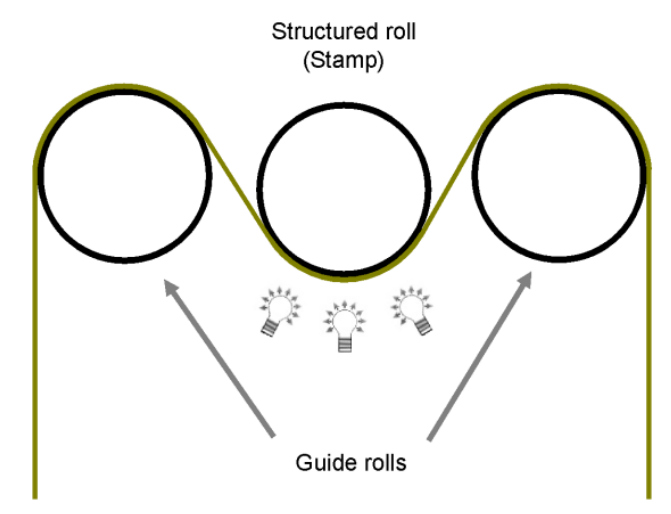

b)

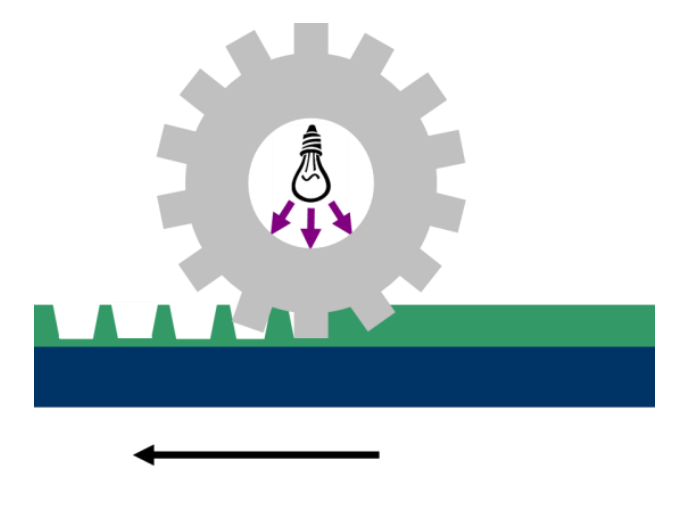

Figure 4.17: Sketch of two different types of roller-based imprint approaches. On the left-hand side a roll-to-roll process that could be used to imprint on flexible foils is visualised. On the right hand side a roll-to-sheet or Roller-NIL principle is visualised. The light bulb inside the roll shows schematically how UV-curable resists on opaque sheets could be patterned. The arrow indicates the moving direction of the substrate. A tapered shape of the patterned resist is indicated. This effect can occur when trying to replicate a binary profile in a Roller-NIL process; however, the dimensions of the stamp's pattern and the diameter of the roller are not to scale.

The demands listed above were taken into account for the design of the roller NIL tool conducted within the framework of the diploma thesis of S. Schwarzkopf 
[172]. The setup consists of a conveyor belt with a wafer carrier fixed on it. The fixing of the wafer is achieved using a vacuum chuck. The maximum speed of the conveyor belt and thus the wafer transport was first chosen to be about $0.5 \mathrm{~cm} / \mathrm{s}$. This velocity was the result of a conservative assessment, guessing potential filling times and necessary exposure doses. By a modification of the gear box driving the conveyor belt, its speed can easily be increased later on. The transparent silicone stamp is mounted on a quartz glass roll to allow the exposure through the stamp. As sketched in Figure 4.17 b), the UV-light source is implemented within the roll. The source itself consists of 18 high performance UV-Light Emitting Diodes (LEDs) emitting at $365 \mathrm{~nm}$ (i-line). The LEDs are mounted in a linear array to illuminate the contact line beneath the roll as homogeneously as possible over a length of about $200 \mathrm{~mm}$. Since, as already mentioned, the contact time of stamp and resist for this type of NIL might be very short (also depending on the imprinting velocity), it is preferable to obtain high intensities in this contact area to ensure a sufficient cross linking of the resist. The intensity resulting from this setup was increased by focusing the light emitted by the LEDs onto the line contact using a linear Fresnel lens. The setup of the LEDs, a heat sink and the lens is sketched in Figure 4.18. Taking the LED's angular dependency and reflection losses at the interfaces into account, the intensity in the contact area is calculated to be about $140 \mathrm{~mW} / \mathrm{cm}^{2}$. For a speed of $0.5 \mathrm{~cm} / \mathrm{s}$ and an assumed width of the contact of $1 \mathrm{~cm}$, this leads to a dose deposited in the resist of $280 \mathrm{~mJ} / \mathrm{cm}^{2}$.

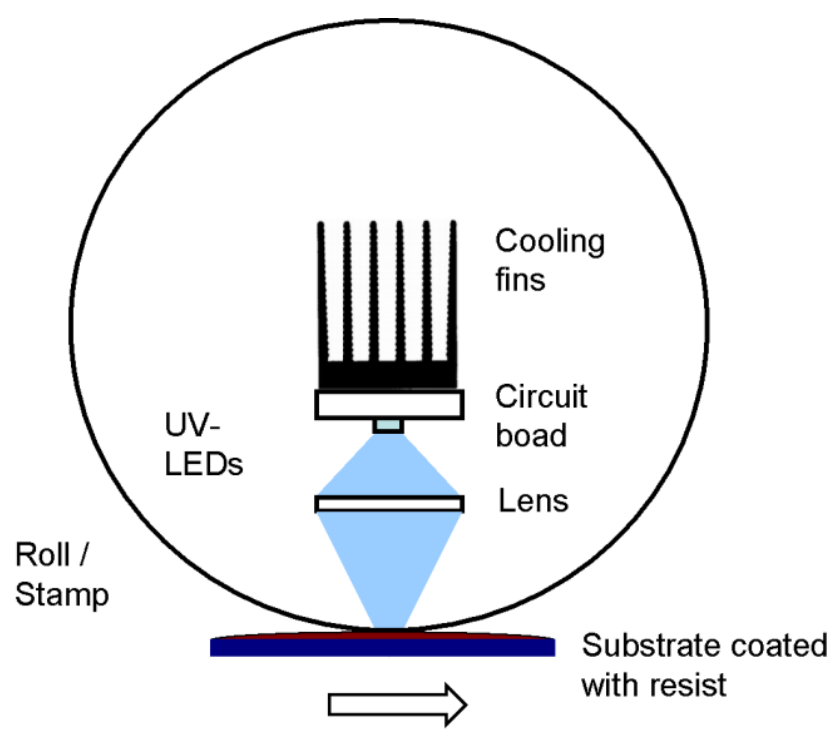

Figure 4.18: Visualisation of the interior of the roll showing the assembly of UV-LEDS, cooling and focusing of the emitted radiation onto the contact area [172].

The roll itself is mounted in a way so as to guarantee a self-alignment to exclude tilting, wedge errors and camber inclination by making use of a ball joint. The tool 
is designed to allow an adjustable embossing force. This is realised by variable forces counteracting the roller's weight. These counteracting forces on both sides of the roll are realised using springs and require a detection of acting forces to enable their calibration (see Figure 4.19). Sensors on each side are used to measure acting forces and to balance the roll. The homogeneity of the pressure distribution is essential for a uniform residual layer thickness in the NIL process.
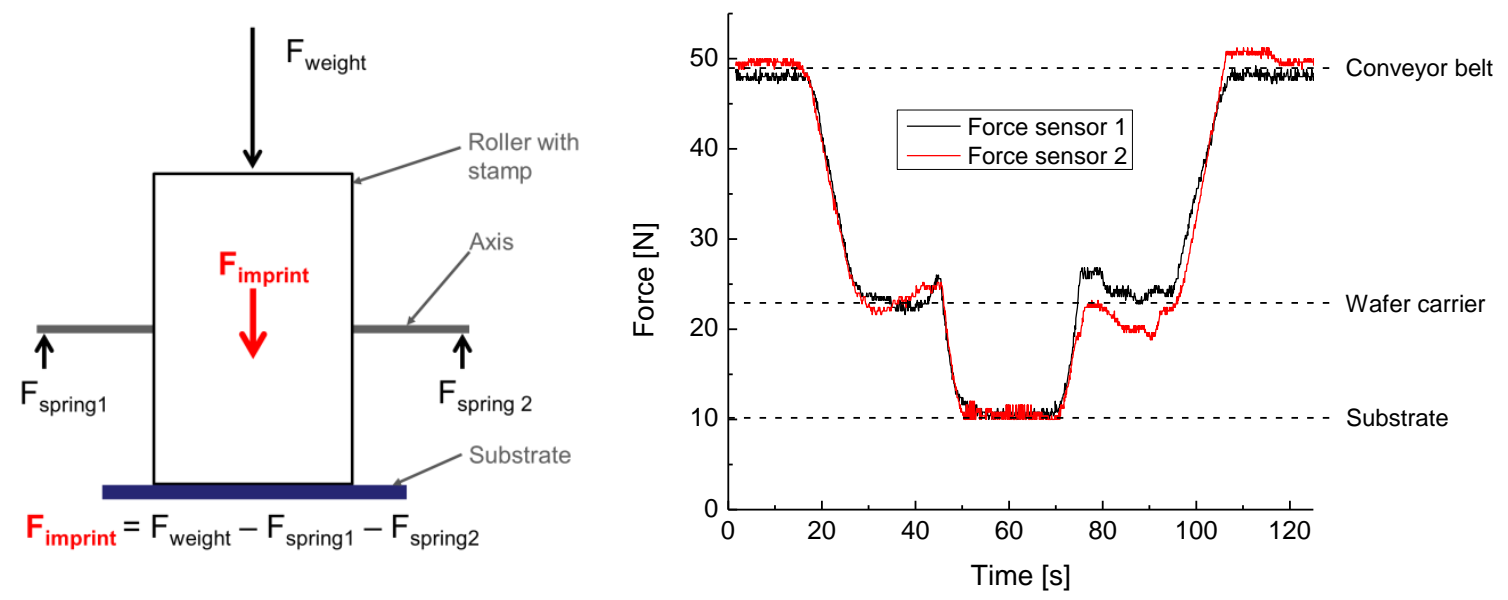

Figure 4.19: On the left hand side, acting forces in the Roller-NIL tool are schematically visualised. The imprinting force can be adjusted by variable forces induced by springs counteracting the roller's weight. These counteracting forces are detected by force sensors as shown in the graph on the right hand side (force sensors: type 8438 from Burster, measurement range from 0 to $100 \mathrm{~N}$ ). There, the forces during different stages of an imprint process are measured. First, the planar silicone belt is rolling over the conveyor belt; then, the roller is lifted onto the wafer carrier and the counteracting force is reduced; then, the roller again is lifted onto the substrate (in this case a glass substrate) and the patterned stamp is rolled over the substrate. During this step, acting forces have to be set properly to obtain a homogeneous imprint result; afterwards, the roller again passes the wafer carrier to the conveyor belt.

Figure 4.20 shows a photograph of the realised Roller-NIL tool imprinting a $125 \times 125 \mathrm{~mm}^{2}$ multicrystalline silicon substrate. 


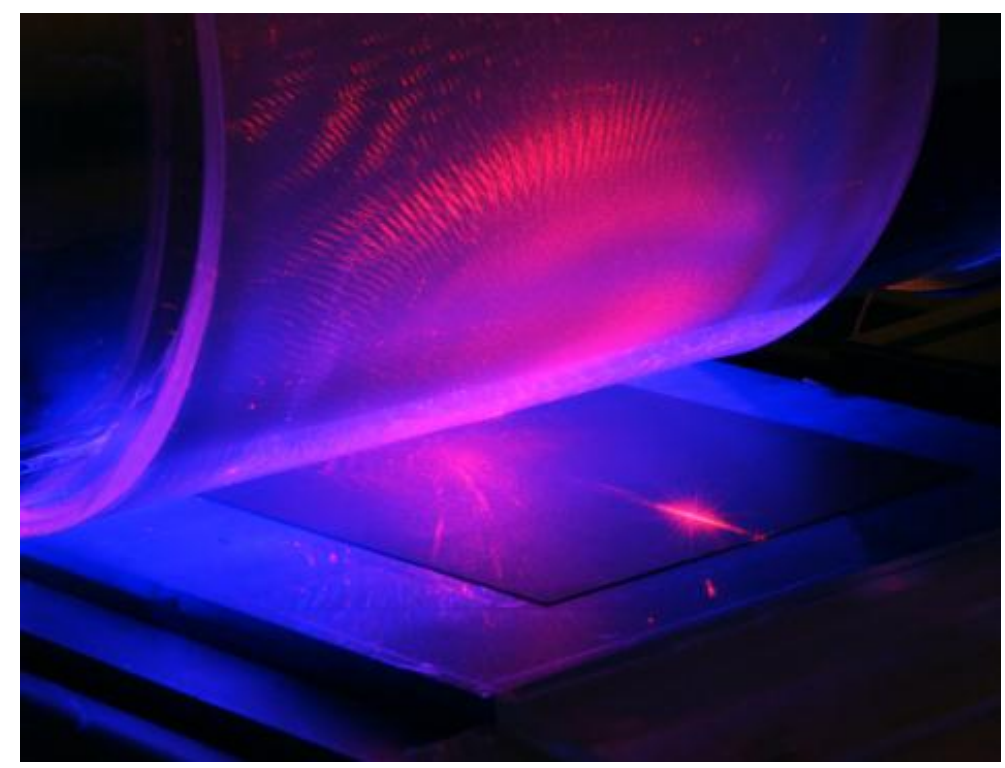

Figure 4.20: Photograph of the Roller-NIL tool processing a $125 \times 125 \mathrm{~mm}^{2}$ multicrystalline silicon substrate. The red light of a HeNe laser is applied to visualise the diffractive features in the stamp.

First tests of the Roller-NIL tool were made using a solvent based resist. The effect of the changing viscosity of the resist due to the outgassing of solvents on the Roller-NIL process was investigated in a similar way as for the conventional NIL tool in the previous section. To this end, a sample was prepared using the optimal conditions for spin coating as extracted from Figure 4.11 (500 rpm spinning velocity). Then, the imprinting was conducted using the Roller-NIL tool. The imprint process along one substrate was conducted piecewise, leaving defined halts of 30 seconds between each section [172]. Figure 4.21 shows the quality of the resulting imprints on three adjacent sections (number 1 being imprinted first and number 3 last). A direct correlation of the resulting pattern depths and residual layer thicknesses as a function of time to the ones obtained with the conventional NIL tool shown in Figure 4.13 is not possible for several reasons (non-homogeneous pressure for static imprinting using the Roller-NIL tool, different holding time before the exposure, a pre-exposure and thus a pre-crosslinking of the imprints number 2 and 3 in the Roller-NIL tool cannot be fully excluded). Still, the qualitative behaviour is in agreement to the results obtained using the conventional NIL tool. 

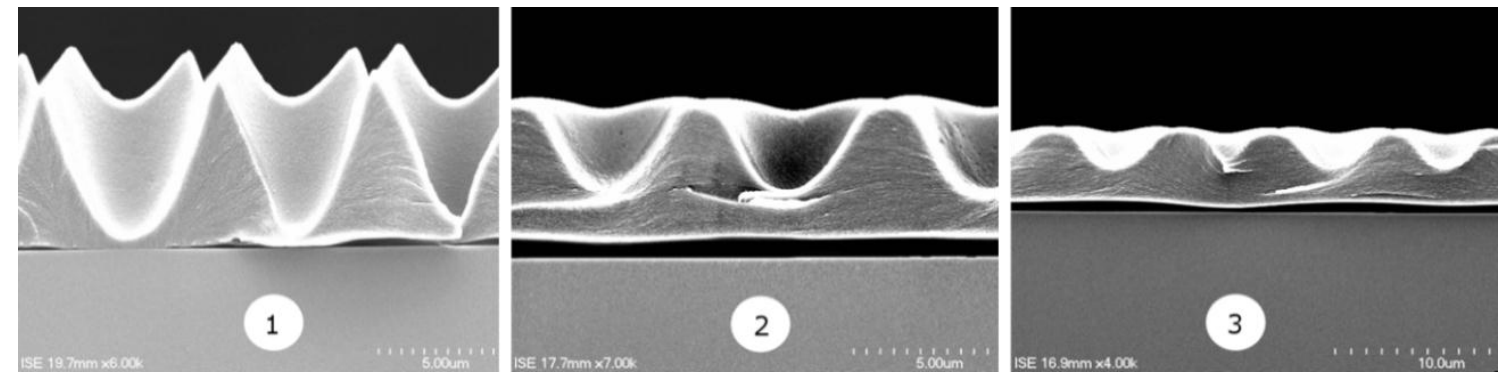

Figure 4.21: SEM micrographs of different parts of a sample, where a solvent based resist is patterned via Roller- NIL. Between these parts, defined halts of the imprinting process were introduced.

The cumulative worsening of the quality of the imprinted pattern after each halt confirms that a solvent assisted NIL process, as developed before for the conventional NIL tool using a planar stamp setup, implies problems for a sequential processing in a Roller-NIL tool. However, there are solutions to that problem so that solvent based resists do not necessarily have to be excluded for this process. First, a different coating technology than spin coating would change the starting position of the degassing along the substrate. An in-line resist coating (e.g. by roller-coating) would allow that each point on the substrate travels the same time from coating to imprinting and thus an initial solvent content could be chosen to meet corresponding requirements. Another option would be to change the type of organic solvent in which the resist is diluted. Solvents of lower gas pressures would help to retard the outgassing process. The methodology to capture the dynamics of the outgassing presented in the section before could then be applied to evaluate different solvent based resist systems. Thermal assisted UV-NIL processes using SU8 as the resist material can also be found in the literature [173]. This would mean that no solvent content would be necessary in the resist. However, in that work, hard stamps and high pressures were utilized, which is contrary to our requirements.

Within this thesis, spin coating was the only available coating technology. Therefore, the Roller-NIL processes were further optimized using solvent-free resists. These were mainly acrylic free-radical polymerising resists of the Laromer product class of BASF. No time dependency on the imprint quality of these resists was found. Figure 4.22 shows the near-zero residual layer thickness on midpoints and near-edge points on a $140 \times 140 \mathrm{~mm}^{2}$ glass sample. This excellent result confirms that a balancing of the roll can be achieved leading to high quality etching masks on large areas. Furthermore, on the full area, the pattern initially originated by interference lithography was replicated with homogeneous pattern fidelity. This shows that, throughout the process chain of master origination, nickel 
replication via electroplating, stamp fabrication via cast moulding and Roller-NIL, patterns can be replicated in a precise and exact way.

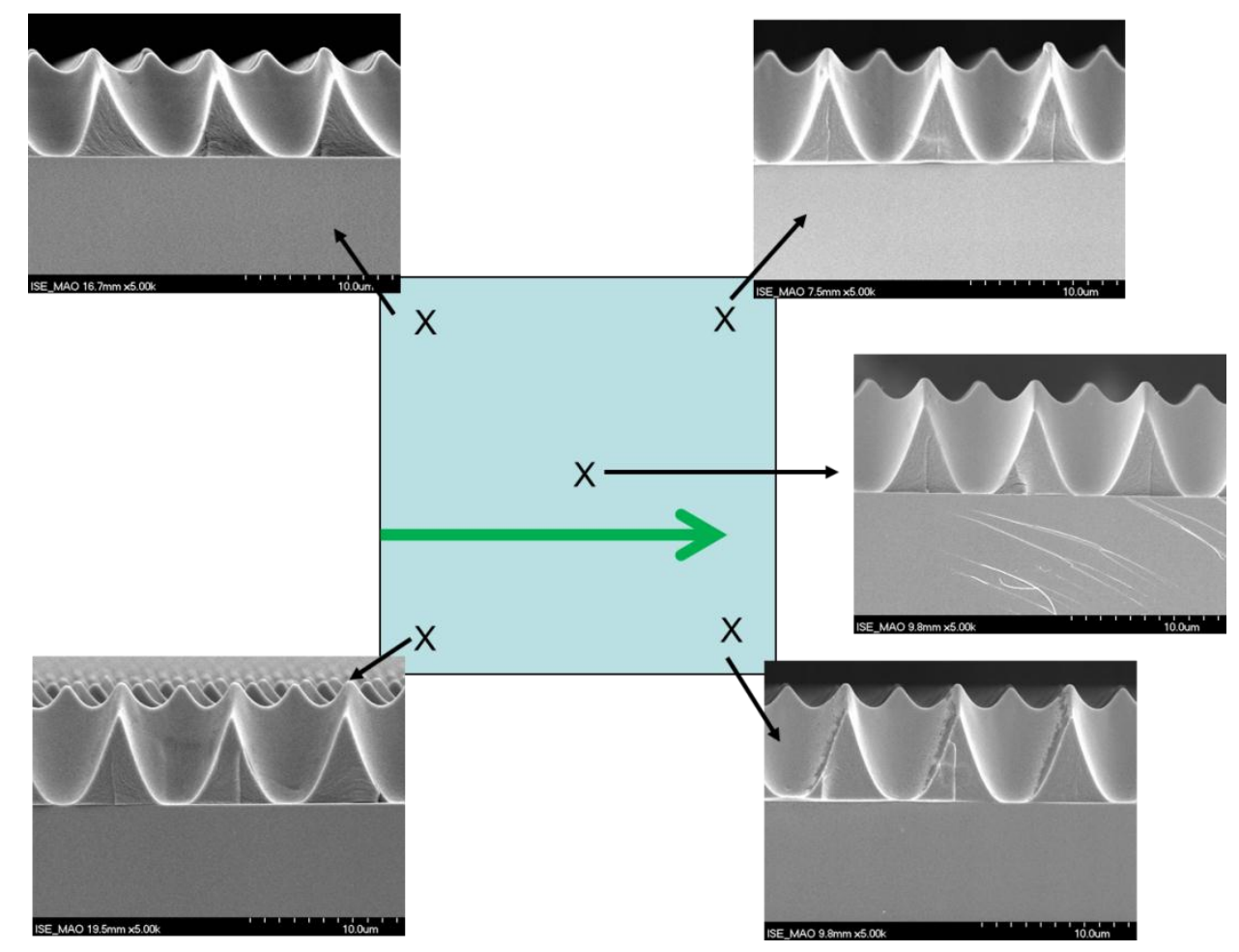

Figure 4.22: SEM micrographs of a Roller-NIL patterned resist layer on a glass substrate. The resist is the solvent-free radical curing Laromer PO84F from BASF. The patterned area is $140 \times 140 \mathrm{~mm}^{2}$. All across the sample, a near-zero residual layer thickness was observed. The micrograph of the slightly distorted pattern in the upper left corner indicates a deformation of the flexible stamp. The big green arrow indicates the moving direction of the sample beneath the Roller-NIL tool.

While the imprint quality using the solvent-free resists of the Laromer product family was found to be excellent, another problem arose concerning the stamp durability. It was found that the adhesion properties of the resist / stamp interface are modified with increasing numbers of imprints. As a result of this modification, the cured resist sticks to the PDMS stamp and is torn off of the substrate. This effect only occurs locally in the beginning, but then evolves to a global phenomenon so that the resist is torn off on the whole area. It is assumed that this results from a monomer or pre-polymer diffusion into the stamp. In contrast to the solvent-based SU8-2002 resist described before, where this effect of a deterioration of the stamps was not found, the low viscosity of the resists is not realised using solvents but results from short polymer chains prior to the curing. The assumption of diffusion effects leading to these degraded lifetimes was further strengthened by testing Laromer types of different molar weight and thus different viscosity. These were namely Laromer PO84F and Laromer LR8996. It was found that the resist with a lower molar weight (Laromer LR8996) led to a faster 
deterioration of the stamp. After approximately 20 imprints, the stamp was not useable any more. Furthermore, SEM micrographs confirmed that polymer residues were sticking to the PDMS surface (see Figure 4.23).
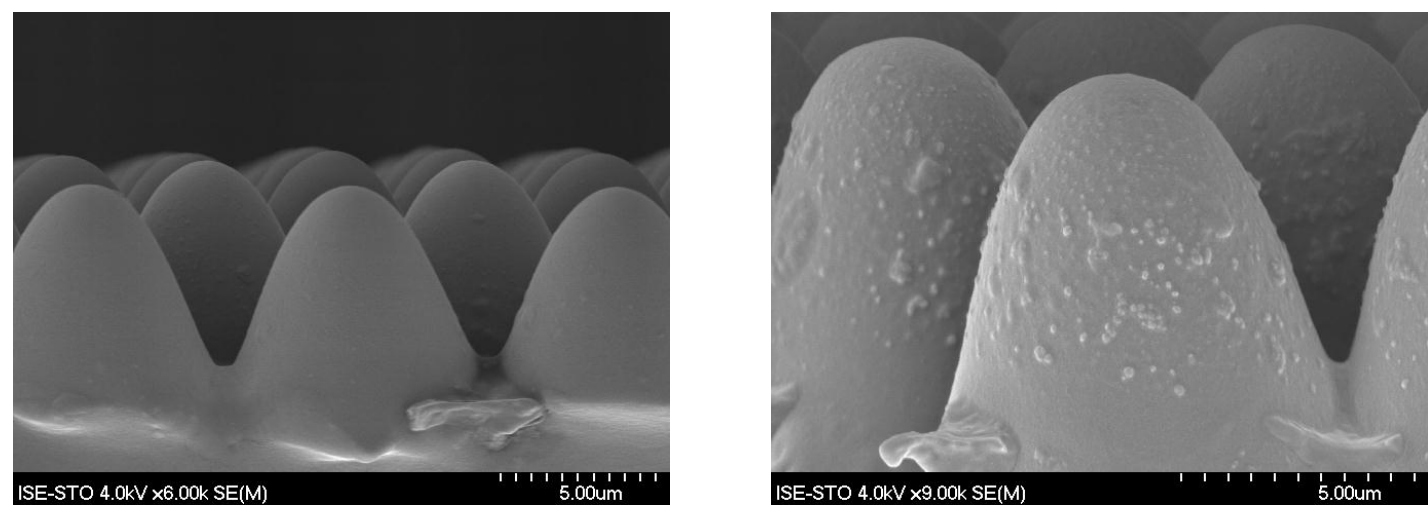

Figure 4.23: SEM micrographs of PDMS stamps before (left) and after (right) degradation by imprinting Laromer LR 8996.

For the Laromer PO84F, the durability is higher (around 50 imprints), but still is not satisfactory. Here, it is important to mention that the PDMS used in this investigation was the Elastosil RT601 from Wacker. The curing of this PDMS was conducted according to the datasheet $\left(50\right.$ minutes at $80^{\circ} \mathrm{C}$ ). Attempts to increase the durability by enhancing the cross-linking of the PDMS via tempering after the curing or modification of the curing process itself led to minor improves. It was found that the shore hardness and thus presumably the cross-linking of the PDMS can be increased slightly. However, the maximum achievable number of imprints using this material combination was below 100 imprints. This is not sufficient to establish industrial scale processes. For the solvent-based resist SU8 described before, no deterioration was found at all for well above 200 imprints. However, this resist system for the current setup of the Roller-NIL tool was only suitable for the conventional NIL tool. Therefore, in future works, the focus will have to be placed on finding suitable material combinations to avoid this deterioration.

\section{Summary of the NIL processes}

In this section, the development of two tools for NIL was described. First, the conventional tool based on a planar stamp setup was constructed and investigations around that tool were preliminary studies for the development of a Roller-NIL tool. As a result of the different stages of development, all structures presented within this thesis realised with the conventional NIL tool were processed with the solvent based resist SU8-2002. The process development using the newly developed Roller-NIL tool required the change of resist systems for the given setup. 
Therefore, all samples processed with the Roller-NIL tool were based on the use of the solvent-free resist Laromer P084F if not declared otherwise.

For the two different types of textures to be realised within the framework of this thesis, different demands were set on the imprint process. For the honeycomb texturing, the focus is placed on processing rough multicrystalline silicon substrates. Furthermore, feature sizes of the hexagonal pattern can be relatively large. The first argument requires and the second argument allows the use of soft PDMS materials for the stamp fabrication. The realisation of diffractive gratings on monocrystalline silicon is more demanding in terms of pattern dimensions. The surface quality of the substrates used for this application within this work is by far better than the one for the honeycomb texturing (bright etched FZ material). Still, NIL processes on full wafer area require the use of flexible stamps to achieve a homogeneous residual layer thickness. To this end, the use of stacked PDMS stamps was established at the Fraunhofer ISE for this application.

For both applications, the honeycomb texturing as well as the realisation of diffractive gratings, first the conventional NIL tool was applied for the process development before transferring the processes to the Roller-NIL tool.

\subsection{Plasma etching processes}

The pattern defined by the etching mask has to be transferred into the silicon substrate. There are potentially two routes which might be followed: (i) wetchemical or (ii) dry chemical approaches. Both might be attractive in combination with imprinted etching masks. Wet-chemical approaches have the advantage of being more or less the standard etching process used in industrial production lines at the moment. Therefore, the introduction of the presented process chain into industrial environments could be easier if wet-chemical etching processes were applied. On the other hand, the application of dry-chemical plasma etching processes allows the fabrication of surface textures with more degrees of freedom concerning the pattern shapes and achievable aspect ratios. Another advantage is the single side treatment, which is of importance when it comes to the introduction of passivated rear side concepts as for the PERC structure. Of course, there are also drawbacks for plasma processes e.g. highly energetic ion bombardment surely enables the generation of deep etching pits with steep sidewalls, but also is related to plasma induced surface damages. However there are also isotropic plasma etching processes, where this negative effect can be avoided, and damaged areas can even be removed.

The focus of the presented work was on plasma etching processes, because they show a higher potential to generate tailor-made high-efficiency textures as already 
demonstrated in [5]. In the following, the basic principles of plasma technology as well as two techniques for plasma etching are explained in detail. These are namely reactive ion etching (RIE) processes, which allow the fabrication of high aspect ratios, and isotropic microwave plasma etching processes, which allow an isotropic etching.

\subsubsection{Basic principles of plasma etching}

The basics about plasma etching described in this work are based on the book of Lieberman and Lichtenberg [160]. The term plasma describes a gas whose atoms are partially ionized. This ionization can be the result of thermal or electrical excitation. The net charge of all particles within the plasma (neutral atoms or molecules, electrons and ions) equals zero. However, there is no sharp distinction between the expressions plasma and gas. In technical applications, artificially ${ }^{13}$ generated low temperature and low pressure plasmas are commonly used for several purposes e.g. etching, deposition, modification of surface properties, etc.... The main characteristics describing a plasma are the following:

- pressure

- temperature

- degree of ionization (also plasma density)

- mean free path-length

Typically, in technical applications, weakly ionized plasma discharges are generated. These types of plasma have the following features: (i) they are generated electrically; (ii) charged particle collisions with neutral gas molecules are important; (iii) there are boundaries at which surface losses are important; (iv) ionization of neutrals sustains the plasma in the steady state and (v) the electrons are not in thermal equilibrium with the ions [160].

\section{Etching technologies}

There are different etching mechanisms applicable using plasma processes. Sputter etching relies on an ion bombardment using inert gases and hence a purely physical ejection of atoms. There, ionized atoms are accelerated onto the sample by an electrical field. The etching rate is mainly dominated by the acceleration voltage, the mass of the ionized etching atoms, the pressure (as this determines the mean free path length) and the material (binding energy and atomic masses) to be etched. For these reasons, sputtering is an unselective etching process since etching rates for different materials only vary in the range of a factor of $2-3$.

\footnotetext{
${ }^{13}$ Natural plasma can be found in stars or lightnings.
} 
Plasma etching processes can also be realized as purely chemical etching processes. In this type of plasma processing, the discharge supplies ions or radicals which can react with surface atoms to be removed. In this case, it is important that the reaction product is volatile, so it can be removed in the gas phase. These plasma etching processes based on chemical reactions are typically of an isotropic nature, unless there are varying etching rates for different crystal orientations of samples to be etched due to different binding energies. An often used process that relies on a purely chemical etching is the ashing of polymeric resists in oxygen plasmas. These polymeric resists mainly consist of long-chain polymers based on carbon and hydroxide. Thus, the chemical reaction with oxygen radicals allows the formation of (predominantly) gaseous phase water and carbon dioxide. In [160], it is also explained that the addition of fluorine based etchants (e.g. $\mathrm{CF}_{4}$ or $\mathrm{C}_{2} \mathrm{~F}_{6}$ ) can help to increase etching rates drastically. This may be related to the formation of $\mathrm{HF}$ when $\mathrm{F}$ radicals react with the polymeric resist. Thereby, a further attack of the resist by oxygen atoms could be facilitated. Other possible explanations of increased etching rates are related to higher densities of atomic oxygen within the plasma in the presence of fluorine.

Besides these two etching processes, it is possible to combine both processes. This combination can be found in the literature under the expressions of ion-enhanced energy driven etching or more often reactive ion etching (RIE). The advantage of this combination of physical ion bombardment and chemical removal of surface atoms is that etching rates can be enhanced significantly when comparing them to those obtained for the single etching mechanisms. However, as a side effect, one has to cope with limits concerning the advantages of these other two processes. A compromise has to be found between anisotropy (which is mainly determined by the physical etching component) and material selectivity (which is related to chemical etching reactions).

Another process that can be found in the literature, is the so-called ion-enhanced inhibitor etching [160]. The idea of this process is that besides etchants, other species are introduced into the plasma that lead to a plasma enhanced deposition. Precursors used for this purpose are e.g. $\mathrm{CF}_{2}, \mathrm{CF}_{3}, \mathrm{CCl}_{2}$ or $\mathrm{CCl}_{3}$ molecules. Thereby, fluoro- or chlorocarbon polymer films can be deposited on the sample. These resistant films then act as an inhibitor layer for the chemical etching. Applying additionally a physical etching component, this masking layer can be broken through. When both effects of deposition and reactive ion etching proceed in parallel, the formation of stochastically ordered needle-like structures can be achieved. This is based on the fact that after an initial abrasion the deposition of an inhibitor layer on the sidewalls of cavities is not removed efficiently by physical means later on. The physical etching is most effective for surfaces orientated 
normally to the incident ion beam. This process is applied for the fabrication of socalled black silicon [76], which reveals excellent reflection reduction properties and thus is very interesting for solar cells [77]. There are slightly different processes which also rely on the formation of an inhibitor layer to increase anisotropy in RIE processes. One well known process is the so-called Bosch process, which is based on alternating RIE and plasma deposition processes. This patented process allows the separate tuning of etching and deposition steps [174]. Fluorocarbon polymer films are deposited as the inhibitor layer. The third means of creating an inhibitor layer is based on the decomposition of the polymeric resist in standard fluorine based RIE processes. Thereby, the plasma composition is changed and a redeposition of fluorocarbon polymers can occur [175]. This effect can be used to realise high aspect ratios.

\section{Fluorine based etching of silicon}

Within the present work silicon etching processes based on the use of sulphur hexafluoride $\left(\mathrm{SF}_{6}\right)$ were applied. The main overall reaction within the etching process of fluorine atoms $\mathrm{F}$ with silicon atoms can be written as

$$
\mathrm{Si}+4 \mathrm{~F} \rightarrow \mathrm{SiF}_{4}(\mathrm{~g}) \text {. }
$$

This total reaction equation can be divided into intermediate reactions. In the following, the intermediate reactions responsible for the silicon etching in RIE processes are listed according to [160]:

Physisorption and thermal desorption of fluorine atoms

$$
\mathrm{F}(\mathrm{g})+\mathrm{S} \leftrightarrow \mathrm{F}: \mathrm{S}
$$

Chemisorption of physisorbed $\mathrm{F}$ atoms at silicon dangling bond $\left(\mathrm{Si}^{*}\right)$ sites

$$
2 \mathrm{~F}: \mathrm{S}+\mathrm{Si}^{*} \rightarrow \mathrm{SiF}_{2}: \mathrm{S} .
$$

lon-induced desorption of $\mathrm{SiF}_{2}$

$$
\mathrm{SiF}_{2}: \mathrm{S} \rightarrow \mathrm{SiF}_{2}: \mathrm{S} \text {. }
$$

Creation of $\mathrm{SiF}_{4}$ by ion beam mixing followed by ion induced desorption

$$
2 \mathrm{~F}: \mathrm{S}+\mathrm{SiF}_{2} \rightarrow \mathrm{SiF}_{4}(\mathrm{~g})+2 \mathrm{Si}^{*} .
$$

Physical sputtering of silicon

$$
\mathrm{Si}^{*} \rightarrow \mathrm{Si}(\mathrm{g})+\mathrm{Si}^{*}
$$

Chemical etching of silicon

$$
2 \mathrm{~F}: \mathrm{S}+\mathrm{SiF}_{2} \rightarrow \mathrm{SiF}_{4}(\mathrm{~g}) \text {. }
$$

Within these equations $S$ stands for the substrate's surface [51]. Besides $S_{6}$ other fluorine based molecules such as carbon tetrafluoride $\left(\mathrm{CF}_{4}\right)$ can be used for silicon 
etching processes. However, due to a lower dissociation energy and a higher amount of fluorine atoms, the etching rates are potentially higher for $\mathrm{SF}_{6}$ [114].

Introducing oxygen to the $\mathrm{SF}_{6}$ can lead to increased etching rates as a result of a higher plasma density of fluorine atoms [114]. This is due to the reaction of $\mathrm{O}_{2}$ with $\mathrm{SF}_{x}{ }^{*}$ or $\mathrm{O}^{*}$ with $\mathrm{SF}_{6}$ forming $\mathrm{SO}_{2}$ and $\mathrm{SOF}_{4}$ respectively. However, for high oxygen concentrations in the plasma, etching rates decrease; this is related to the deposition of a $\mathrm{Si}_{x} \mathrm{O}_{y} \mathrm{~F}_{z}$ film.

A critical issue for etching processes based on $\mathrm{SF}_{6}$, especially for large area markets such as photovoltaics, is the enormous global warming potential (GWP) of this gas. The 100 year GWP of $\mathrm{SF}_{6}$ is 22200 fold that of the same mass of $\mathrm{CO}_{2}$ and its lifetime in the atmosphere is 3200 years [176]. Therefore, gas abatement is very crucial when it comes to the industrial realization of plasma etching processes based on $\mathrm{SF}_{6}$. Some strategies for gas abatement systems are described in [114]. Another approach for maintaining fluorine based etching processes but minimising the GWP of used gases is the application of $F_{2}$ as an etching gas [177]. $F_{2}$ itself has a GWP of zero; however, the synthesis and the disposal are associated to emissions [178]. The main challenges for the technological implementation of this etching gas are related to its corrosive behaviour as a result of the formation of HF. Within the framework of this thesis, solely $\mathrm{SF}_{6}$ was used as a gas for silicon etching since only this was available in the laboratory scale tool. An up-scaling of the etching processes to industrial scale should be conducted using $F_{2}$. This was not further investigated within this thesis.

\section{Plasma etching tool applied within this work}

The development of plasma etching processes within this thesis was conducted using a laboratory scale plasma cluster consisting of a chamber for etching processes and one for PECVD processes. The description of this tool, schematically shown in Figure 4.24 is based on the works of Lüdemann and Schäfer [78, 179]. 


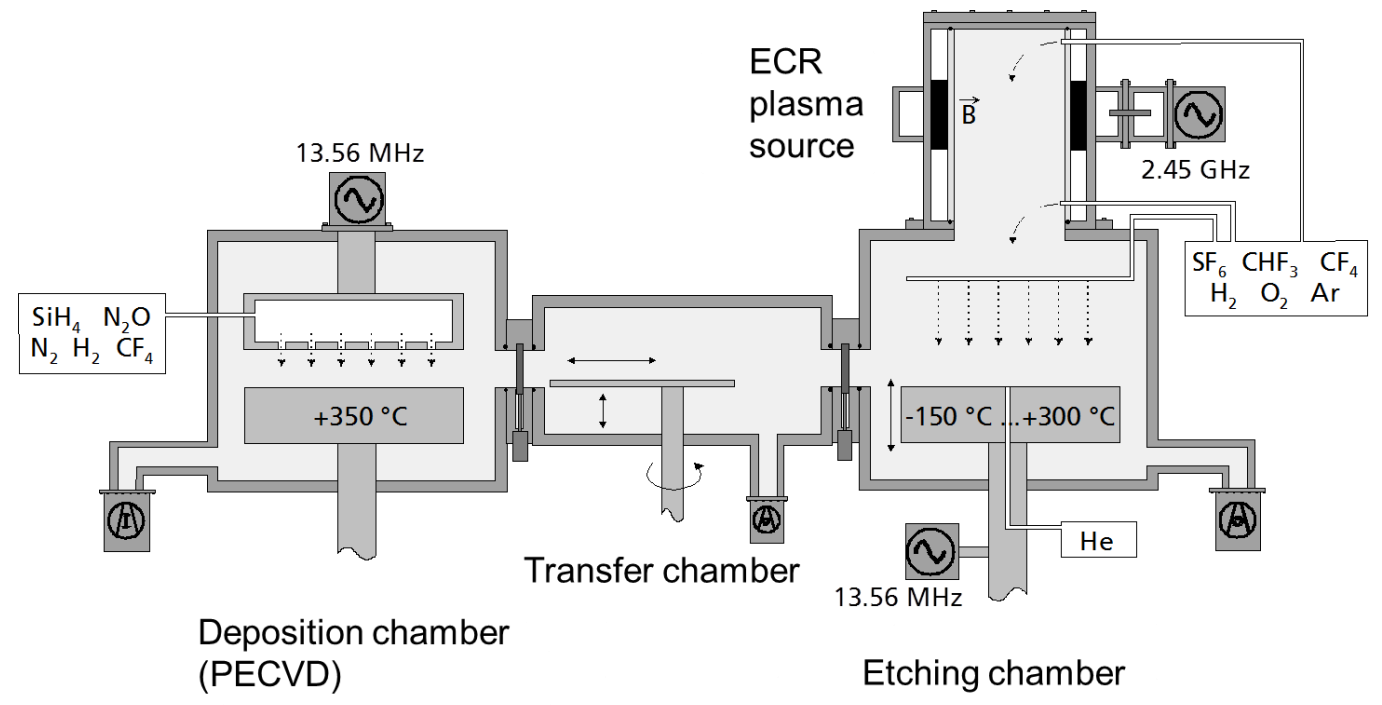

Figure 4.24: Setup of the plasma cluster for etching (right) and PECVD processes (left). A handling system connects both chambers. Graphic taken from [179].

Within this work only the plasma etching tool shall be described, since active development was only pursued therein. The radio frequency driven PECVD chamber was applied e.g. for the deposition of silicon nitride passivation layers or silicon oxide dielectric buffer layers, but only already existing standard processes were applied. The setup of the etching chamber allows the use of very different plasma etching conditions. This is related to different types of excitation sources. The radio frequency driven parallel plate setup allows the capacitively coupled excitation of gases for the use of RIE processes. As a second option, a microwave (MW) slot-antenna (SLAN) source is implemented. An overview of other potentially applicable plasma sources for PV applications was published by Schäfer et al. in [180]. In the present tool, there are two different inlets for the gases to be used. One is implemented just above the sample holder, and the other above the microwave SLAN source. The lower electrode on which the sample is placed can be elevated and its temperature can be adjusted. For a better thermal coupling of the lower electrode, it can be purged with Helium from the backside.

The maximum possible plasma density is proportional to the square of the excitation frequency [78]. Therefore, the $2.45 \mathrm{GHz}$ microwave source allows the realisation of higher plasma densities than the $13.56 \mathrm{MHz}$ RF source. The SLAN setup realised in the present tool additionally features a permanent magnet as indicated by the magnetic field $\vec{B}$ in the sketch in Figure 4.24 . When the magnet field is perpendicular to the electrical field, a more efficient incoupling of energy into the plasma can be achieved. Thereby, for low pressures, the so-called electron-cyclotron-resonance (ECR) can be realised. In this case, very high energetic 
electrons lead to a high density of ionised atoms. The advantage of microwave plasma excitation is that purely chemical etching processes are possible, since no acceleration voltage has to be applied as is the case for capacitively coupled plasmas. By combining these two power sources, the RF source can be used to tune an ion bombardment independently of the adjusted plasma density set via the MW source.

\subsubsection{Plasma etching for silicon photovoltaics}

There are several potential areas of application for plasma processes in solar cell fabrication. Both etching and deposition processes can be used to realise sophisticated solar cell concepts.

For the etching processes, there are different areas of application in solar cell processing. Besides the relatively obvious applications of saw damage removal and texturisation processes, there are also applications in the fields of dry chemical cleaning processes, selective removal of the PSG layer and edge isolation processes $[114,181]$.

There are also concepts relying on a fully plasma based fabrication of solar cells. To this end, all texturing and cleaning processes [176] as well as deposition of diffusion sources [182] can be applied via plasma processes. Advantages resulting from plasma etching compared to wet-chemical etching typically listed in the literature are: (i) single side treatment; (ii) independency of crystal orientations (in particular for mc-Si); (iii) less critical wafer handling; (iv) less consumption of deionised water; (v) no toxic chemical waste (especially for HF based etching processes). Critical issues related to plasma etching for solar cell fabrication are greenhouse gas emissions, throughput, maintainance and the necessity of vacuum technology. To the knowledge of the author of this thesis, up to now there is only one solar cell company using plasma etching based texturisation processes. In the scientific publications of Kyocera it can be found that they are working on RIE processes based on chlorine chemistry [183]. These processes are based on selfmasking effects by depositing an inhibitor layer, as described for the ion-enhanced inhibitor etching before, and are applied for the texturisation of mc-Si. Besides the mainly economical or ecological aspects mentioned up to now, another key aspect for introducing plasma etching processes into solar cell fabrication is the assurance of high material quality after the plasma treatment. Therefore, in the following, some aspects of plasma induced harms relevant for silicon solar cells will be explained. 


\section{Plasma induced deterioration of electrical properties of silicon}

There are various mechanisms of plasma etching processes, which can lead to enhanced loss mechanisms in solar cells later on. In particular RIE processes, where ions are accelerated onto the silicon substrate, can be crucial in terms of creating crystal defects or introducing contaminations.

Crystal defects as a result of the ion bombardment can be formed in terms of a breakage of chemical bonds or a restructuring of silicon atoms, which is more likely [179]. Contaminations can occur as a result of fast ions hitting the reactor wall or sample holders and so ejecting atoms like e.g. $\mathrm{Fe}, \mathrm{Ni}, \mathrm{Cr}$, Al or others. These atoms then can be ionised, thus forming part of the plasma, and subsequently be accelerated onto the sample. Thereby, defects can be introduced that lead to the creation of recombination sites, leading to increased Shockley Read Hall recombination. Another negative effect is related to harsh UV-radiation emitted from the plasma. These highly energetic photons can lead to breakage of chemical bonds and thus also create crystal defects. In [111] this radiation effect was investigated and it was found that even for samples with a dielectric passivation layer, there was a degradation of lifetimes due to RIE treatment. However, for these presumably radiation induced damages, a reversibility was demonstrated.

The effect of deposition processes occurring in parallel to etching processes is critical when it comes to the passivation of plasma processed silicon. Before, the deposition of fluorocarbon polymer films was described as positive effect for the realisation of high aspect ratio features and steep sidewalls. However, when it comes to the passivation of texturised samples, such films can deteriorate surface recombination properties. Therefore, either optimised plasma processes or wetchemical cleaning procedures have to be applied to ensure a complete removal of these films.

In Figure 4.25 these negative effects are visualised using a model introduced by Lüdemann [179] and Schäfer [78]. In this model, it is assumed that, depending on the process, a thin layer of non-volatile reaction products can be deposited on the silicon wafer. Directly beneath the silicon surface, a layer with heavy damages can be found up to depths of 10 to $20 \mathrm{~nm}$. At depths up to 50 to $80 \mathrm{~nm}$ there is a considerable amount of introduced contaminants leading to this layer being named modified silicon. Even deeper regions are affected by the diffusion of voids. These defects can be found up to depths of 1 to $1.5 \mu \mathrm{m}$. 


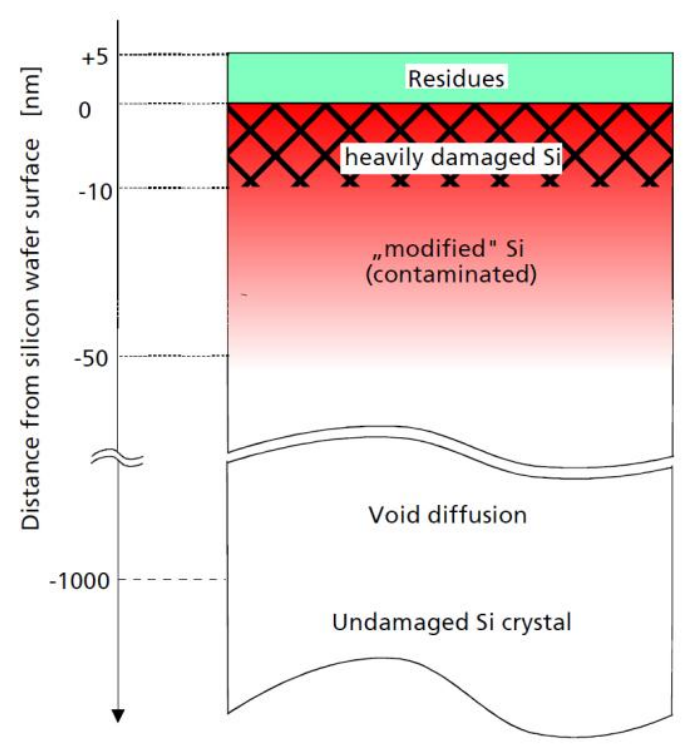

Figure 4.25: Sketch of plasma induced effects in silicon as a function of the distance to the silicon surface. This model was introduced by Lüdemann [179] and Schäfer [78]. The graphic is taken from [51].

\section{Summary of the plasma etching processes}

Plasma etching allows the realisation of well-defined textures independently of crystal orientations. Therefore, it is very well suited for both types of textures to be realised within this work. The different dimensions of these types of textures require different processing parameters in terms of etching rates and anisotropy. For the honeycomb texturing very high etching rates must be achieved, since several microns of silicon have to be removed. Contrarily, the realisation of diffractive gratings requires small etching rates to end up with reproducible etching depths in the $200 \mathrm{~nm}$ regime. It was mentioned that the anisotropy can be enhanced by a decomposition of polymeric resist masks. However, processes to achieve this are very sensitive and therefore cannot necessarily be transferred from one resist to another.

Besides these aspects, the introduction of damages caused by the plasma treatment was described. As the concepts investigated within this work are related to the fabrication of high-efficiency solar cells, processes have to be established that introduce as little damages as possible. This can be realised by minimising acceleration voltages in RIE processes, which can be counterproductive when it comes to the realisation of highly anisotropic profiles. Another way to minimise the influence on the electrical properties of the solar cell is introduced in section 5.2. There, an additional layer is patterned on top of a passivated silicon wafer. 


\section{Two concepts based on defined textures}

In this chapter two concepts are presented that aim for an improved optical behaviour of silicon solar cells by introducing defined textures via NIL. First, a micron scale texture shall be applied on multicrystalline silicon solar cells, namely the honeycomb texture. The second, more sophisticated concept is the realisation of photonic structures on the wafer rear side. The solar cell processing of NIL textured substrates was done in cooperation with the department for solar cell development and characterisation at the Fraunhofer ISE. Solar cell processes for the honeycomb textures were conducted in collaboration with Bernhard Michl and for the rear side gratings with Jan Benick.

\subsection{Honeycomb textured multicrystalline silicon solar cells}

\subsubsection{Description of this concept}

As explained in chapter 3 , the benefit of a designed texture on the front surface is especially pronounced for mc-Si. The highest efficiencies for this type of material were achieved applying the so-called honeycomb texture $[5,89]$. There, a hexagonal pattern is etched into the silicon surface using conventional photolithography. This fabrication method is suitable for the laboratory scale but the applied process chain cannot be transferred to industrial environments.

The technology of NIL, especially when implemented as a Roller-NIL process, has the potential to allow a cheap patterning of etching masks and thus to bring this high-efficiency texture closer to an industrial realisation. Concerning the structure dimensions to be realised, optical simulations led to the conclusion that the period of this texture should be somewhere above $5 \mu \mathrm{m}$ [184]. This results from the relatively broad spectral range $(\sim 300-1200 \mathrm{~nm})$ within which this texture shall be active to achieve both a reduction of front surface reflectivity as well as a path length enhancement in the solar cell. For smaller dimensions, wavelength-selective diffractive effects become more dominating. When looking at the practicability of this concept using NIL, a trade-off has to be met for the structure dimensions. Larger periods are easier to fabricate, in particular on rough substrates as is necessary for industrially applied mc-Si; however, it is desirable to achieve high aspect ratios in the following etching process. This implies that the etching time as well as the amount of silicon to be removed via etching increases for larger patterns. We tried to meet these demands by choosing a period to be $8 \mu \mathrm{m}$, which 
is well above the $5 \mu \mathrm{m}$ criterion given by the optical simulations and is feasible for NIL. Also, for soft stamp materials like PDMS, which has to be applied on rough substrates, these dimensions are not critical, as we are aiming for the aspect ratio of the etching mask pattern to be around one.

The shape of the hexagonally arranged etching pits is important for the optical behaviour of the texture. Within the diploma thesis of Marcel Pfeiffer [184] wave optical simulations were conducted to formulate some design rules for this shape. The modelling method applied for these calculations, is the rigorous coupled wave analysis (RCWA) [185]. Simulating bi-periodic structures with large periods using RCWA is computationally demanding; the profiles were therefore simulated as uniperiodic grooves as opposed to bi-periodic wells. The results should hence be interpreted as a comparison between types of structures, since absolute values may not be representative of the bi-periodic case. Furthermore, the calculations are done for one single wavelength of $680 \mathrm{~nm}$, which should be reasonable for a comparably large structure period of $8 \mu \mathrm{m}$ in terms of most likely minor wave optical effects. Figure 5.1 shows the calculated reflectivity as a function of the aspect ratio for the three profile types. These are namely a prismatic (in 2D this would correspond to a conical shape), a parabolic and an elliptical profile of the 1D grating. It can be seen that the prismatic profile performs best. In the following, based on the assumption that the results of this 1D simulation qualitatively also hold for the 2D case, some design rules for the honeycomb texture are extracted.

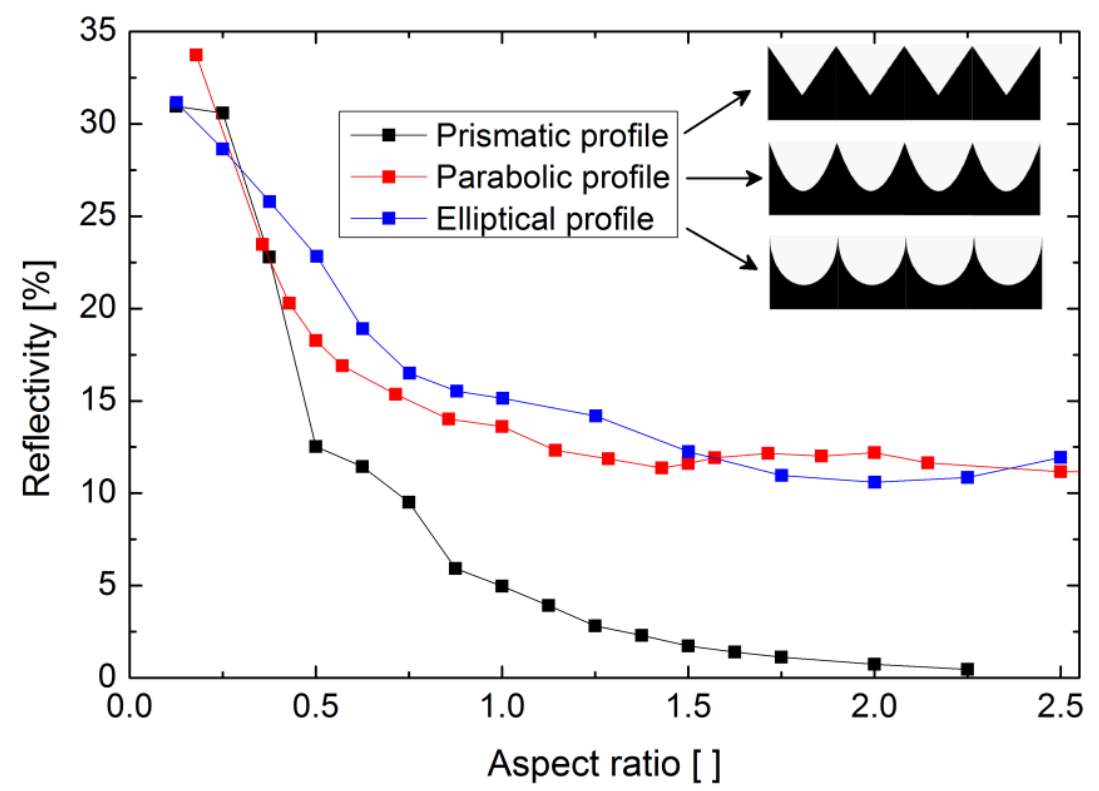

Figure 5.1: Wave optical simulation of reflectances for linear gratings for varying aspect ratios and distinct profile shapes etched into silicon. The results are obtained for a single wavelength of $680 \mathrm{~nm}$. Data is taken from [184]. 


\section{Summary of the design rules for the honeycomb texture and implications on the optimum etching mask pattern:}

- The period of the hexagonal pattern is chosen to be $8 \mu \mathrm{m}$.

- The prismatic (or in other words conical) shape is best: However, this most likely is not practicable in realisation for mc-Si.

- Looking at the more feasible shapes of elliptical and parabolic profiles, the aspect ratio should be above 1 . There, it can be seen that the parabolic shape is superior to the elliptical one and for both of them most of the potential gain is already reached.

- These first aspects can be put together in a statement: minimise flat areas at the bottom of the etching pits. This obviously also holds for the top regions of the pattern. The hexagonal shape of the honeycomb texture can also be related to this context, since etching pits are arranged in the densest packing order.

- To minimise (or even to avoid) flat areas in the resulting texture, it would be desirable to generate infinitesimal hole openings in the etching mask. The reason for this is: unmasked areas will typically remain flat after the etching process. Trying to replicate needle-like structures with sub-micron diameter and potentially necessary heights of above $2 \mu \mathrm{m}$ (to realise the desired aspect ratio later on) with soft stamp materials to generate very small hole openings, would be extremely demanding and not a stable and easy process. However, in NIL it is very easy to replicate continuous profile shapes. Therefore, we aim for an etching mask layout that is continuous and has a preferably small opening in the bottom regions.

- A continuous profile shape can later be beneficial when it comes to the upscaling of the NIL to a Roller-NIL process. The continuous demoulding process induces peeling forces, which might be very critical considering a binary profile.

- The pattern of the etching mask should not have too small an aspect ratio, so that we do not require an etching resistivity that is too high. Therefore, we aim for an imprinted pattern depth of about $8 \mu \mathrm{m}$ (equals to an aspect ratio of about 1 ).

In the following, the realisation of honeycomb textured mc-Si solar cells via NIL will be described. Each process step will be explained in detail.

\subsubsection{Interference lithography and stamp fabrication}

As explained in section 4.2, in interference lithography, expanded laser beams are superimposed on a photoresist coated sample. The hexagonal pattern necessary 
for the honeycomb texturing can be generated by a superposition of three laser beams. A more detailed description of the interference lithography using three beams to originate the hexagonal patterns as used in this work can be found in [127]. The period of the resulting pattern is determined by the angles of incidence on the sample of the laser beams. As shown in [143], for a symmetric setup of the three incoming beams (same polar angle $\alpha$ and azimuth angles of $0^{\circ}$ and $+-120^{\circ}$ ), the period $\Lambda$ can be calculated via

$$
\Lambda=\frac{2 \lambda}{3 \sin (\alpha)} .
$$

This equation can be derived in analogy to the reciprocal lattice formalism as demonstrated for the superposition of two waves in [58]. The requirements on the etching mask design formulated before have to be considered already for the master fabrication, since here the pattern of the stamp and thus the resulting pattern of the etching mask is determined to a certain degree. It was stated that we aim for pits with a continuous profile and a preferably tapered base. Further on, the pattern depth in the imprinted etching mask should be about $8 \mu \mathrm{m}$; therefore, the pattern in the master structure should be at least about $9 \mu \mathrm{m}$. In practice it should be deeper because each replication step that will follow the master fabrication might induce some loss in structure depth. To meet these demands, the positive tone photoresist $A Z 9260^{\mathrm{TM}}$ was chosen. This resist allows relatively thick films to be processed, in which continuous profiles can be transferred lithographically. A critical issue is the drying of thick films of this resist; this is described in [127]. In that work, the desired pattern was fabricated on an small area $\left(75 \times 75 \mathrm{~mm}^{2}\right)$. Within this work, master structures on sample sizes of $250 \times 250 \mathrm{~mm}^{2}$ were fabricated.

A resist layer was applied via spin coating with a film thickness of $17 \mu \mathrm{m}$. Beneath the resist layer, a chromium oxide layer was deposited, both for suppression of reflections at the resist/glass interface during the exposure as well as for adhesion promotion of the resist layer. An offset exposure is conducted to later on remove the so-called surface inhibition layer in the development step as described e.g. in [186]. This surface inhibition layer is only weakly soluble in the developer solution. The formation of this layer is related to the drying, and thus the solvent content, of the resist. The net dose applied in this step was $243 \mathrm{~mJ} / \mathrm{cm}^{2}$. After this exposure step, the resist is partially chemically modified so that regions that were exposed become soluble in an alkaline developer solution. The development process was conducted at room temperature using the developer AZ 400K ${ }^{\mathrm{TM}}$, which was diluted with deionized water at the rate of 1:3, respectively. 
After the development process, a photoresist surface relief remains on top of the glass substrate as shown in Figure 5.2. The period is around $8 \mu \mathrm{m}$ and the pattern depth is around $9 \mu \mathrm{m}$ (measured from the bottom to the tips of the pits). As already explained in section 4.2 these photoresist masters can be repetitively replicated via electroplating (most often in nickel), leading to a family tree of socalled shims.
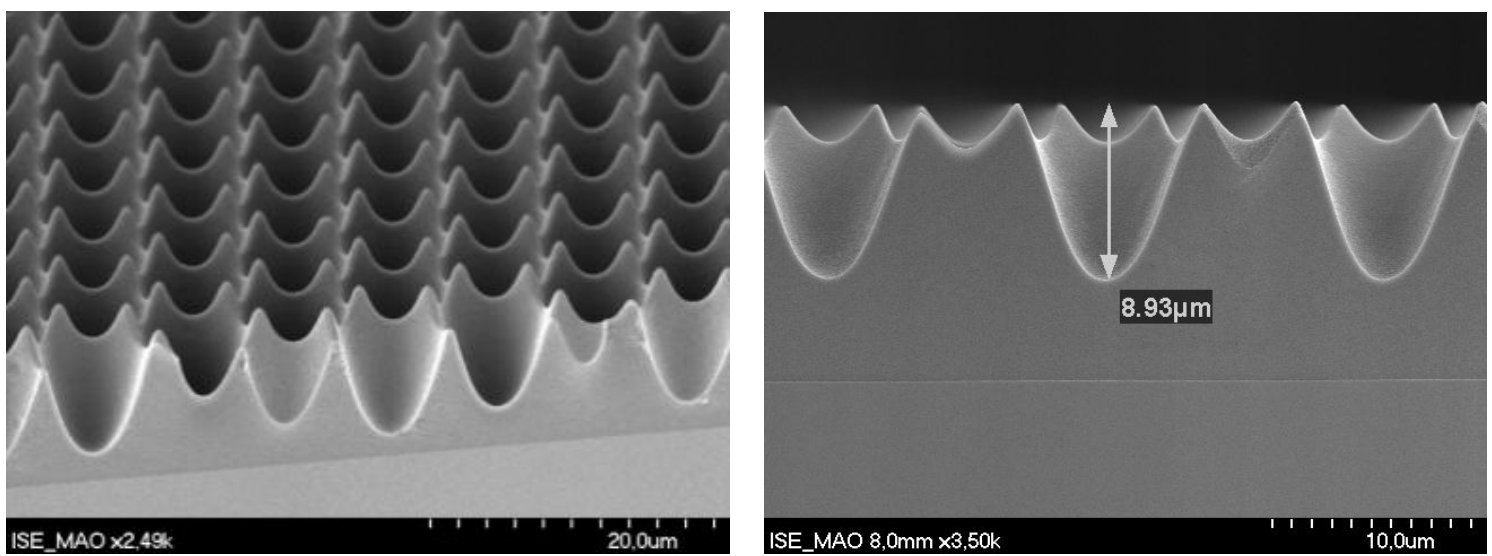

Figure 5.2: Hexagonal surface reliefs in a positive tone photoresist on top of a glass substrate. On the left hand side a tilted view and on the right hand side a cross-section view from SEM micrographs are shown.

The nickel shims fabricated by electroplating can then be used as a template for stamp fabrication. For these relatively large structures, commercially available PDMS materials from Wacker (Elastosil RT601) or DowCorning (Sylgaard 184) have been tested successfully. We used these two-component addition curing PDMS types, which were processed by cast moulding and thermal curing. Depending on the NIL tool for which stamps are fabricated, the processing is slightly different. For both tools presented within this work, the liquid PDMS is poured into a frame onto the template. For the conventional NIL tool based on a planar stamp setup, a quartz plate is coated with a primer (DowCorning 92-023) and the coated side is pressed onto the liquid PDMS. Then, during the thermal curing process, the PDMS is chemically bonded to the quartz glass substrate. In the fabrication of stamps for the Roller-NIL tool, a PDMS belt is formed, which is later fixed mechanically onto the quartz roll. Figure 5.3 shows an SEM image of the resulting pattern in PDMS. It can be seen that the hexagonal pattern is replicated with high pattern fidelity. This PDMS replication was found to be homogeneous on large areas up to $18 \times 20 \mathrm{~cm}^{2}$. 


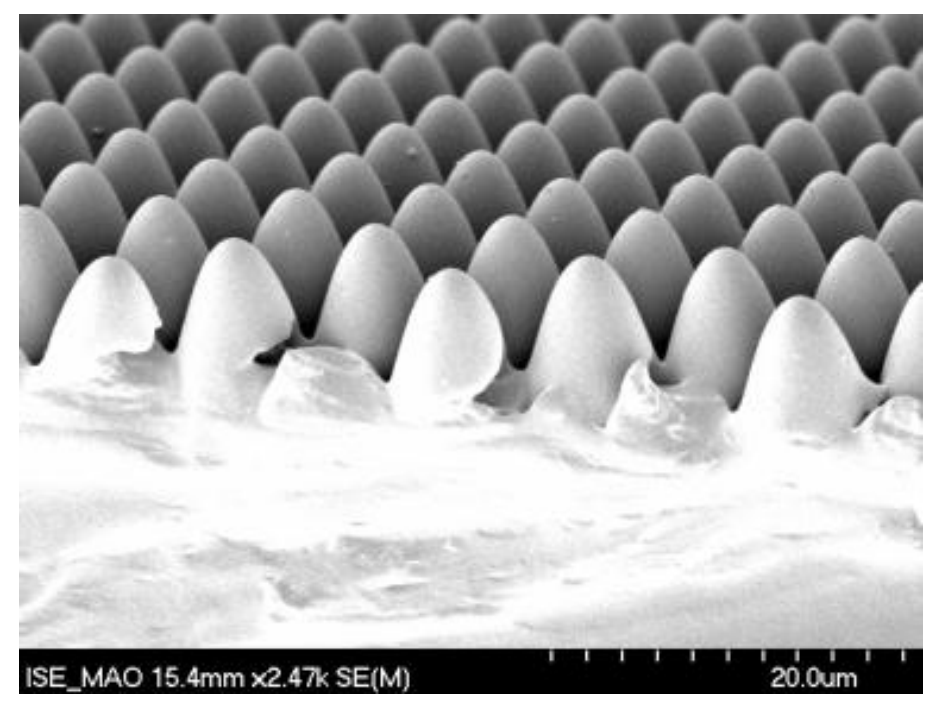

Figure 5.3: SEM micrograph of a replication in PDMS of the hexagonal surface relief fabricated by interference lithography, electroplating and subsequent cast moulding.

\subsubsection{Nanoimprint Lithography (NIL)}

In the process step of NIL, first proof-of-concept investigations were conducted using the conventional tool for NIL developed within this work, in which honeycomb textured solar cells were realised on small areas. In parallel to these works, the up-scaling to a large-area Roller-NIL process was conducted. There, it turned out that the processing is completely different compared to the conventional tool. This is mainly due to the different types of resist materials that have to be applied. To understand the implications for the following process steps, both process developments are described separately.

\section{Conventional NIL}

The development of the processing conditions for this process was already explained in subsection 4.3.3. The PDMS stamp, whose fabrication was described in the previous section, is bonded to a quartz glass substrate. This substrate acts as carrier within the NIL tool. The maximum substrate size that is applicable in this tool is $100 \times 100 \mathrm{~mm}^{2}$. All process development using this NIL tool was first conducted using the solvent based cationic curing negative tone resist SU8$2002^{\mathrm{TM}}$. Table 5.1 summarises the process conditions for the patterning of an etching mask on $100 \times 100 \mathrm{~mm}^{2}$ substrates. 
Table 5.1: Processing conditions for the spin coating and the NIL process.

\begin{tabular}{l|l|l}
\hline \hline Process & Process sequence / Purpose & Conditions \\
\hline \multirow{3}{*}{ Spin Coating } & Remove dust or particles & $4000 \mathrm{rpm}$, acceleration time $5 \mathrm{~s}, 10 \mathrm{~s}$ \\
\cline { 2 - 3 } & Apply resist / Spread & $50 \mathrm{rpm}$, acceleration time $5 \mathrm{~s}, 10 \mathrm{~s}$ \\
\cline { 2 - 3 } & Spinning & $500 \mathrm{rpm}$, acceleration time $10 \mathrm{~s}, 15 \mathrm{~s}$ \\
\hline \multirow{3}{*}{ NIL } & $\begin{array}{l}\text { Pressure / Holding time before } \\
\text { exposure }\end{array}$ & $0.6 \mathrm{bar} / 1: 30 \mathrm{~min}$ \\
\cline { 2 - 3 } & Exposure /Curing of the resist & $\begin{array}{l}1: 30 \mathrm{~min} \text { (resulting Dose at } 365 \mathrm{~nm}: 100 \\
\mathrm{~mJ} / \mathrm{cm}^{2} ; \text { explanation in the text) }\end{array}$ \\
\hline \hline
\end{tabular}

Prior to the resist application, the wafers are coated with an adhesion promoter. This material is spin-coated and afterwards baked. As depicted in section 4.3.3, the NIL process using this solvent-based resist is very time critical and shows a very narrow processing window. Therefore, just after the spin coating, the NIL process has to be started immediately. During the holding time (time between applying pressure onto the stamp until the start of the exposure) it can be seen that in the beginning there is typically trapped air and/or degased solvent beneath the stamp. This air bubble decreases in size gradually until it vanishes completely. This effect nicely shows the effect of gas permeability of PDMS. After all air inclusions vanish, the exposure is started. A broad band illumination is conducted using a Xenon lamp. The intensity is measured using a commercially available UV-meter at a narrow spectral range around $365 \mathrm{~nm}$ (Lutron UVA-365, see also section 4.3.3). This measured intensity is used to calculate the exposure duration to deposit a dose of about $100 \mathrm{~mJ} / \mathrm{cm}^{2}$. As the light source emits in a broader range then the measured one, the total dose will be higher; however, for the negative tone resists applied within this work, an over exposure is not critical. After the curing of the resist, the substrate is demoulded from the stamp. This process step is realised manually. Figure 5.4 shows resulting imprints on c-Si as well as mc-Si substrates. The pattern depth of the imprinted features is slightly reduced to about $8 \mu \mathrm{m}$ (for the master structure it was about $9 \mu \mathrm{m}$ ). However, this is not too surprising looking at the several replication steps in between. Finally, during the imprint process using a soft stamp material like PDMS deformation effects furthermore decrease the pattern depth. Still, the imprinted resist pattern shows a high quality and the design criteria formulated in the beginning of this chapter are fulfilled. These are mainly a low residual layer thickness, an aspect ratio of around one and very small hole openings of the mask when applying the plasma etching. 

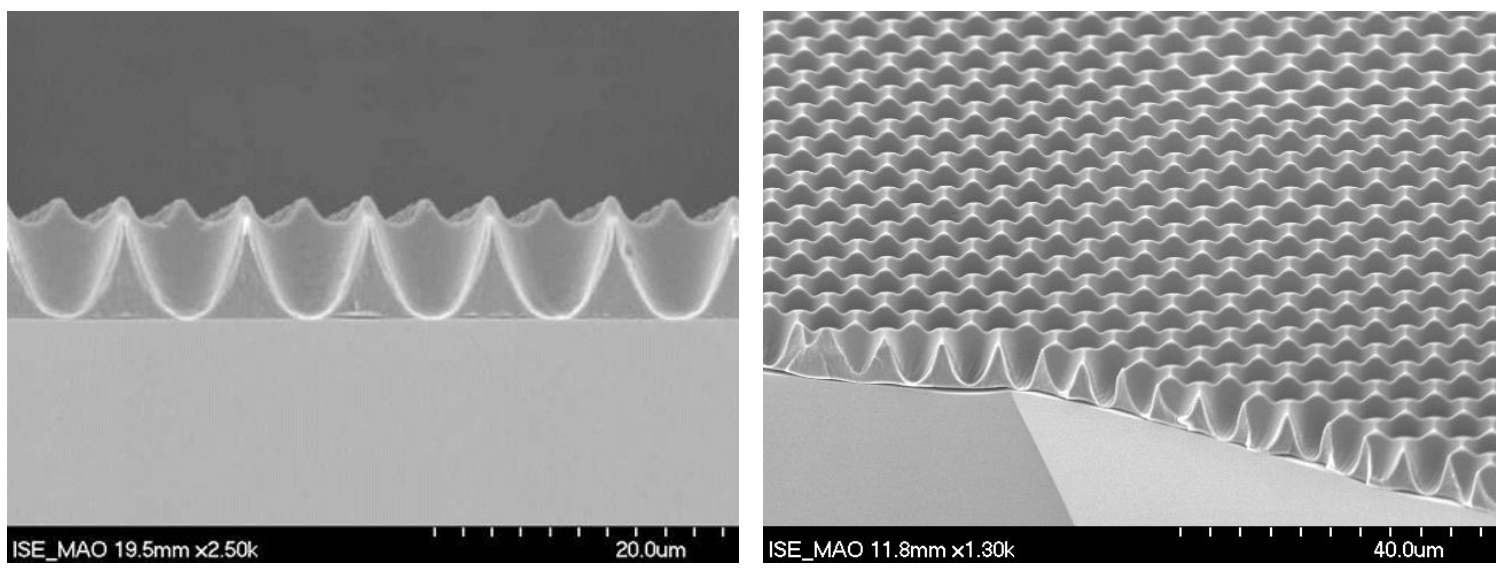

Figure 5.4: SEM micrographs of via conventional NIL patterned etching masks on a c-Si (left) and a mc-Si (right) substrate.

\section{Roller-NIL}

As described in section 4.3.3 a different resist material than for the conventional NIL tool has to be applied. For the works described in this chapter, the solvent-free radical curing material Laromer P084F from BASF was used. Again, the resist was applied via spin coating. Unlike as for the conventional tool, no adhesion promoter was necessary for the applied resist system. However, it was also tested for the purpose of using it as sacrificial layer. Compared to SU8, the solvent-free Laromer offers a wider processing window. The resist has to be applied in the appropriate thickness, so that for a complete filling of the stamp's cavities there is ideally no residual layer. When measuring the spin-coated resist layer thickness, one would have to cure the resist in an inert gas atmosphere to achieve reliable measurements. This results from oxygen inhibition effects that occur for this radical polymerising type of resist. Therefore, it can be more convenient to optimise the initial resist layer thickness by investigating the imprint result. Thus, the residual layer thickness can be applied as a figure of merit to assess the initial layer thickness. Figure 5.5 shows a graph with measured residual layer thicknesses for specific spinning durations at 4000 rotations per minute. 


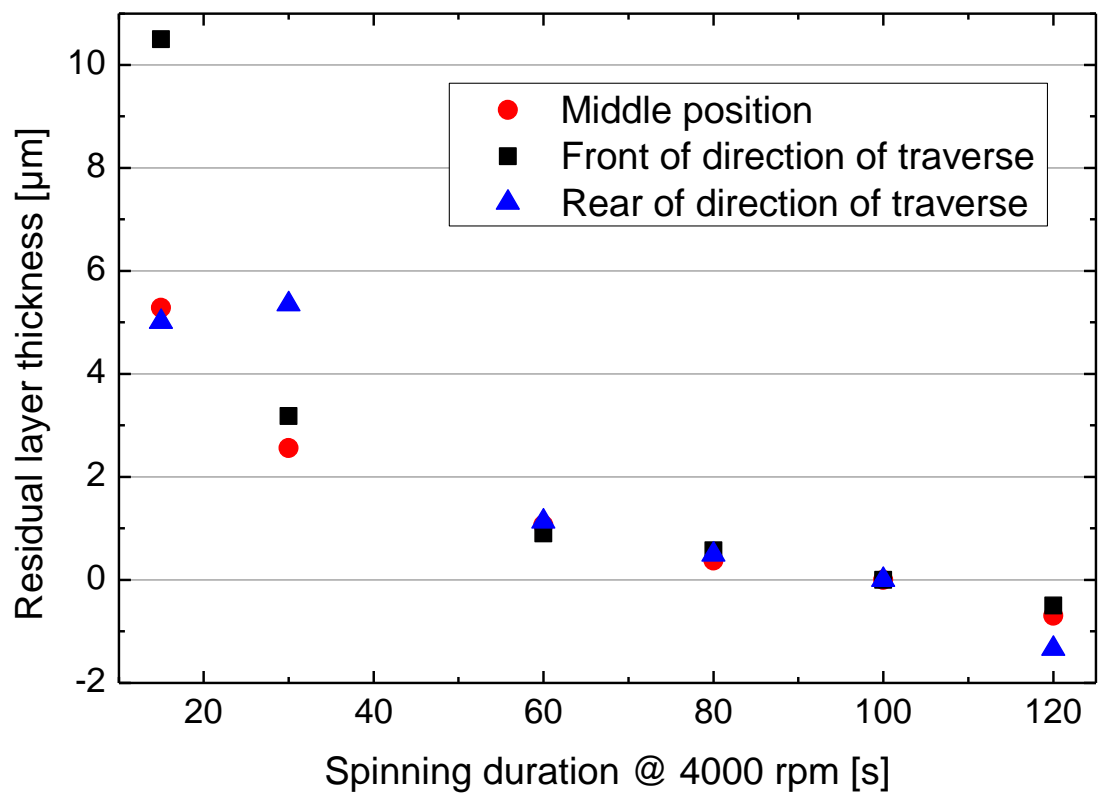

Figure 5.5: Residual layer thickness obtained after the Roller-NIL process using Laromer PO84F spincoated for different times at a fixed spinning velocity. Thicknesses were measured on different positions on the sample. The meaning of negative values is explained in the text.

In the graph in Figure 5.5, the residual layer thickness is determined by measuring the total resist thickness from the top of the resist pattern to the resist/glass interface and then subtracting a fixed pattern depth. The fixed pattern depth was determined as the maximum achieved using this particular stamp. As can be seen, the optimum spinning duration is $100 \mathrm{~s}$ at 4000 rotations per minute. There, virtually no residual layer can be found in the SEM analysis. The negative values for the residual layer thickness for longer spinning durations indicate that, for thinner initial resist layers, the stamps cavities are not filled completely, which leads to a deformation of the stamp's pattern (for better understanding, see the right SEM micrograph in Figure $5.6 \mathrm{c})$ ). This study was conducted on three positions on the sample.

For the Roller-NIL process, the most important parameters are the velocity of the conveyor belt, the power of the UV-LEDs emitting at $365 \mathrm{~nm}$ (and thus the resulting intensity) and the pressure (as a result of acting forces and contact area between stamp and substrate). There is an interaction between the velocity of the conveyor belt and the LED power, as both determine the deposited dose for the curing of the resist. Within this work, no optimisation concerning the maximum velocity for the imprinting was investigated. The applied dose was about $900 \mathrm{~mJ} / \mathrm{cm}^{2}$ for the lowest velocity of $1.5 \mathrm{~mm} / \mathrm{s}$, so that a complete curing of the resist could be assumed. The imprinting pressure of about 0.6 bar results from a resulting force of the roller of $110 \mathrm{~N}$ and a contact area beneath the stamp of 
about $18 \mathrm{~cm}^{2}$. Figure 5.6 shows SEM micrographs of imprinted patterns into resist layers of different thicknesses.

a)

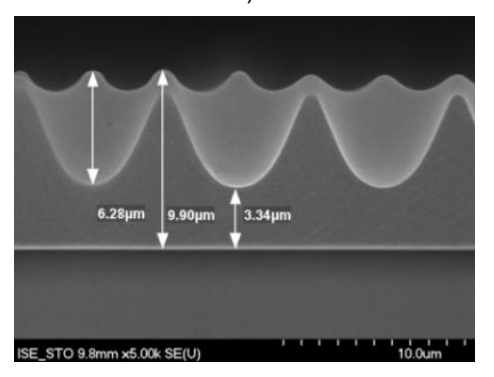

b)

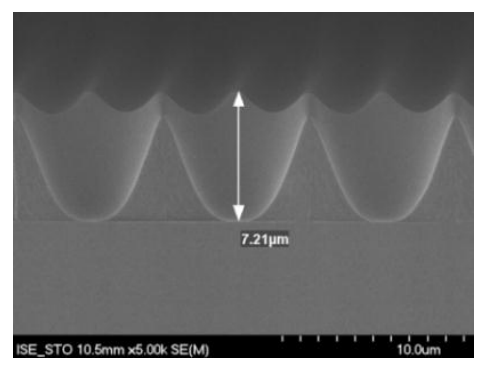

c)

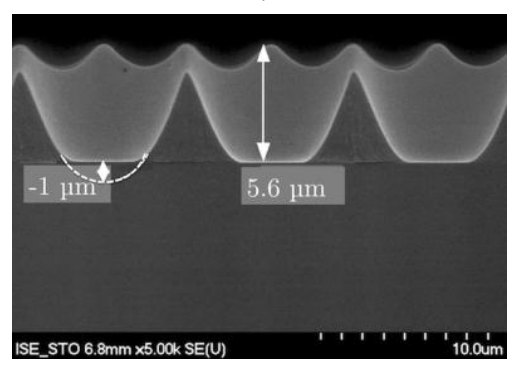

Figure 5.6: SEM micrographs of via Roller-NIL patterned resist layers on glass substrates. From left to right the initial resist thickness is decreasing, leading to different residual layer thicknesses as well as structure depths and widths.

For overly thick resist layers, the residual layer is too thick, so that an etching process later on cannot start immediately (Figure 5.6 a)). For overly thin resist layers, the structures of the soft stamp are deformed leading to increasing hole openings in the etching mask (Figure $5.6 \mathrm{c}$ )). This is not favourable, because as indicated before this leads to flat areas in the resulting texture after the etching processes. Therefore, an optimum exists for the resist thickness before the imprinting process (Figure 5.6 b)). However, this only holds for silicon wafers with very smooth surfaces. When patterning resist layers on rough mc-Si substrates, a compromise has to be met. Applying the optimum layer thickness as determined for a smooth sample, there are considerable areas in which the residual layer will be too thick to allow a pattern transfer. Decreasing the resist thickness leads to a minimisation of this effect; however, the diameter of the hole openings due to an elastic deformation of the stamp's structures is increasing. Both effects are visualised in Figure 5.7. 

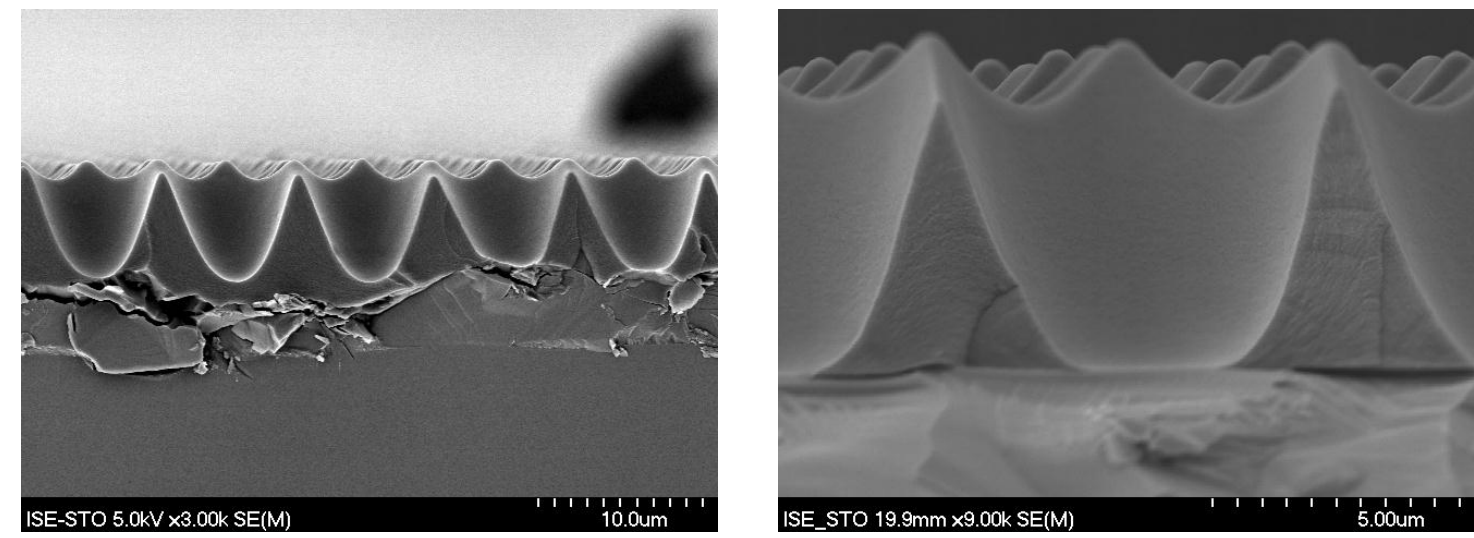

Figure 5.7: SEM micrographs of Roller-NIL patterned resist layers on rough as-cut mc-Si substrates. On the left hand side, the resist layer thickness was applied via spin coating according to parameters that were optimised on a perfectly planar surface. On the right hand side, a thinner layer was applied. This leads to a homogeneous residual layer thickness, but also to widened hole openings in the etching mask.

In Figure 5.8 the excellent adaptability to rough surfaces is visualised for a via Roller-NIL patterned etching mask on a rough mc-Si substrate. There, as a test to evaluate the adaptability of the stamp, the as-cut surface after the wire sawing process was not removed. A compromise in the spin-coated resist thickness allows the realisation of a homogeneous residual layer thickness without introducing too strong deformations in the stamp.

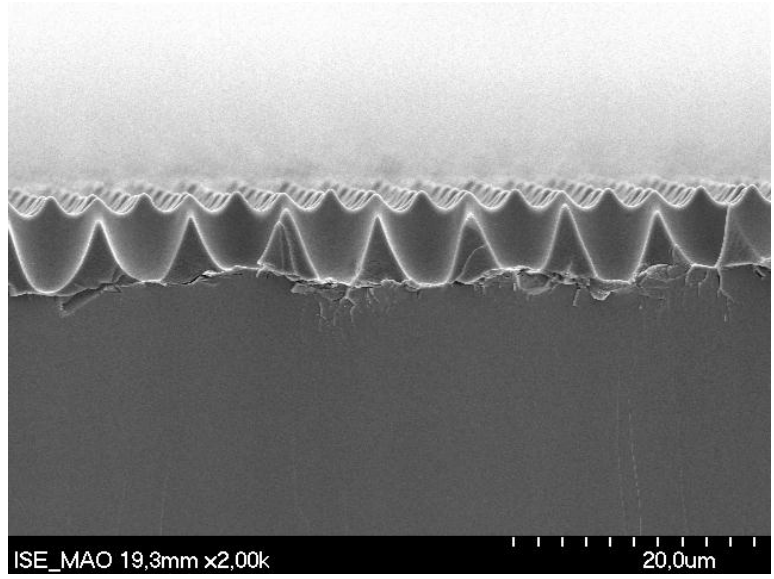

Figure 5.8: Roller-NIL structured polymer layer on top of a rough mc-Si substrate. The hexagonal patterned polymer layer has a very low residual layer thickness (polymer thickness beneath the depressed areas), which is essential for the following etching processes. For this sample, the patterned substrate size was $100 \times 100 \mathrm{~mm}^{2}$.

The newly developed Roller-NIL tool allows the patterning of such etching masks on industrial scale substrate sizes of $156 \times 156 \mathrm{~mm}^{2}$. Issues that have to be tackled next are finding a resist coating process suitable for an integration into the RollerNIL tool thus allowing a fully in-line patterning of etching masks. Other 
optimisations will be related to processing velocities and are very important to increasing the durability of the stamps. These topics shall be investigated in future works.

\subsubsection{Plasma etching and resist removal}

The plasma etching processes for the pattern transfer of the honeycomb texture are oriented to the ones developed in [21]. There, a two-step plasma etching process was applied, which amongst other developments led to the efficiency record for mc-Si. Within this work, the two-step etching process has been complemented by a resist removal step in between. In the first place, a reactive ion etching (RIE) process is used to generate deep, isolated etching pits with steep sidewalls. After this RIE process, resist residues are removed. For this purpose, both wet-chemical as well as plasma-based approaches are investigated. The second etching step is based on an isotropic microwave (MW) plasma process. In this step, the etching pits are widened until they merge and planar areas between them vanish. Furthermore, this very smooth etching process is applied to remove potentially introduced plasma-damaged regions close to the surface. Figure 5.9 visualises this process sequence of etching and resist removal.
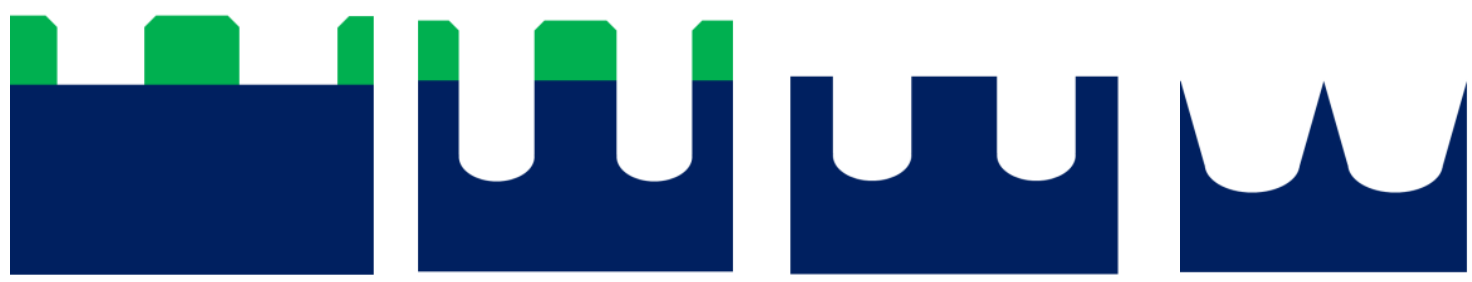

Figure 5.9: Sketch of the steps for the pattern transfer starting with a masked silicon wafer. In a first step, an anisotropic reactive ion etching (RIE) process is conducted. In the second step, resist residues are removed. In a final step, an isotropic microwave plasma etching process is applied to widen the etching pits.

The etching processes and the resulting textures are afterwards assessed in terms of profile shapes, and thus resulting optical quality, as well as surface recombination velocities of the processed samples. Measurements of topography are done by SEM or optical microscopy. The optical properties of textured substrates are investigated using Fourier spectroscopy. Electrical properties are captured via quasi-steady-state photoconductance (QssPC) [187] or photoluminescence measurements.

\section{Reactive Ion Etching (RIE)}

The RIE processes developed in this work are conducted in a parallel plate reactor, were the RF power is coupled capacitively into the process chamber (see section 4.4). As the etching gas, sulphur hexafluoride $\left(\mathrm{SF}_{6}\right)$ was used. First, the influence of 
adding oxygen $\left(\mathrm{O}_{2}\right)$ to the $\mathrm{SF}_{6}$ on the etching rate was investigated as described in [188]. A variation of the ratio of these gases as well as the total gas flow was conducted. Additionally, the effect of the vertical position of the lower electrode, on which the substrates are lying, was studied (with a lower level meaning a larger distance between the electrodes). The pressure in the plasma chamber was kept fixed at $20 \mathrm{~Pa}$ to achieve high etching rates with low bias voltages. The power of the plasma source was kept constant at $300 \mathrm{~W}$. These investigations were conducted on bright-etched FZ substrates, on which an SU8 resist layer was imprinted using the conventional NIL tool with the parameters as listed in Table 4.2. The etching rate was then determined using an SEM. The resulting etching rates for these variations are shown in Figure 5.10.

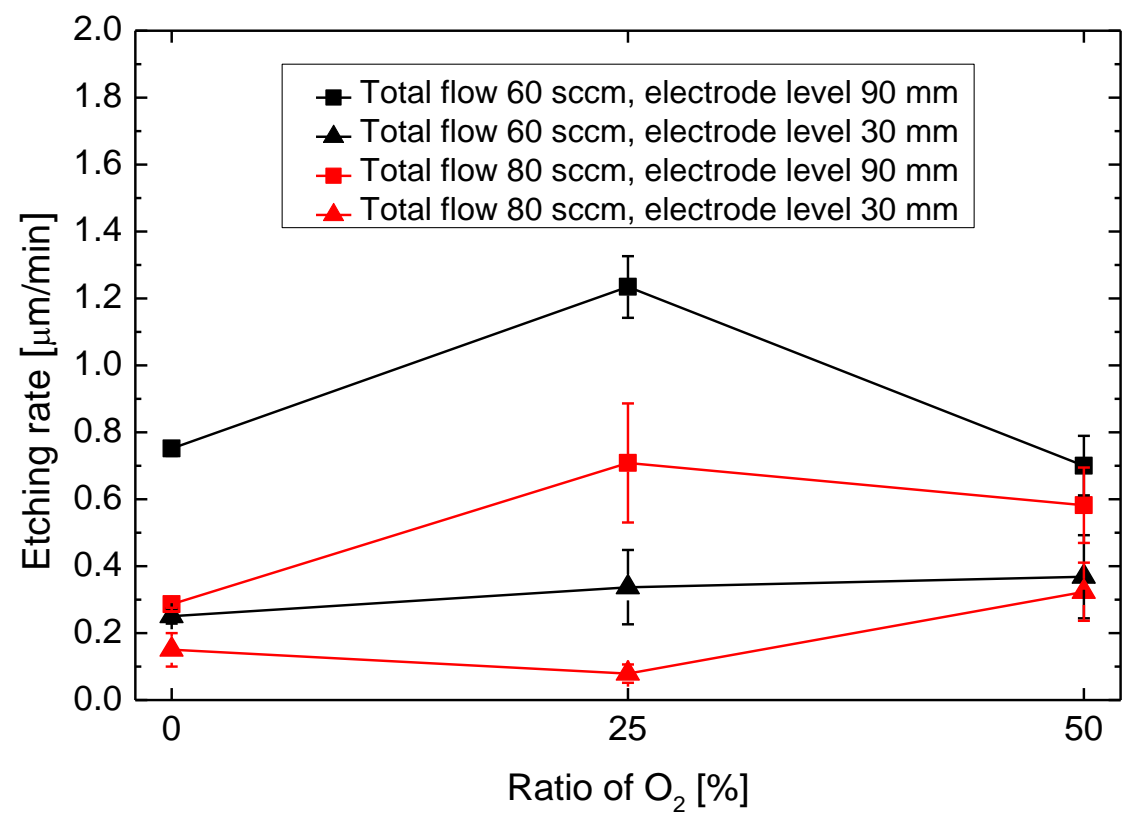

Figure 5.10: Influence of the ratio of $\mathrm{SF}_{6}$ and $\mathrm{O}_{2}$ as well as two different levels of the lower electrode on the etching rates in RIE. The electrode level is a machine specific dimension.

In this figure, it can be seen that a maximum of the etching rates of $1.3 \mu \mathrm{m} / \mathrm{min}$ can be achieved for a total gas flow of $60 \mathrm{sccm}\left(15 \mathrm{sccm} \mathrm{O_{2 }}\right.$ and $\left.45 \mathrm{sccm} \mathrm{SF}_{6}\right)$ and a higher level of the electrode (a smaller distance between the plates). That the etching rate rises and then falls on increasing the oxygen is in agreement to [114]. This effect was explained in section 4.4.

As the next step, potentially occurring plasma damages to the front surface were investigated. Unmasked samples were etched using the presented process variation (FZ, $p$-type, $1 \Omega \mathrm{cm}, 250 \mu \mathrm{m}$ thick). Afterwards, a phosphorus emitter diffusion was applied, leading to a sheet resistivity of $125 \Omega / \square$. The samples were passivated by a $10 \mathrm{~nm}$ thick thermal oxide and the minority carrier lifetimes were 
determined to extract values for $S_{\text {front }}$ in order to calculate the emitter saturation current density $j_{0} e$ as described in [188]. Furthermore, the values of $j_{0 e}$ allow the calculation of the implied limit to the open-circuit voltage $V_{o c}$ as it is limited by the recombination in the emitter and the textured front as described by equation (2.16), which is a rearrangement of the diode equation. In a real device, $j_{0}$ consists of $j_{0 e}$ as well as the recombination in the bulk $j_{0 b}$ (and potentially in the back surface field $\left.j_{0 b s f}\right)$. In the calculation of $V_{o c}$, it is assumed that recombination only occurs in the emitter and on the front surface, and therefore $j_{0}$ equals $j_{0 e}$. Figure 5.11 shows values for the implied limit of $V_{o c}$ as a result of the investigated etching processes for the assumption of a $j_{s c}$ of $38 \mathrm{~mA} / \mathrm{cm}^{2}$.

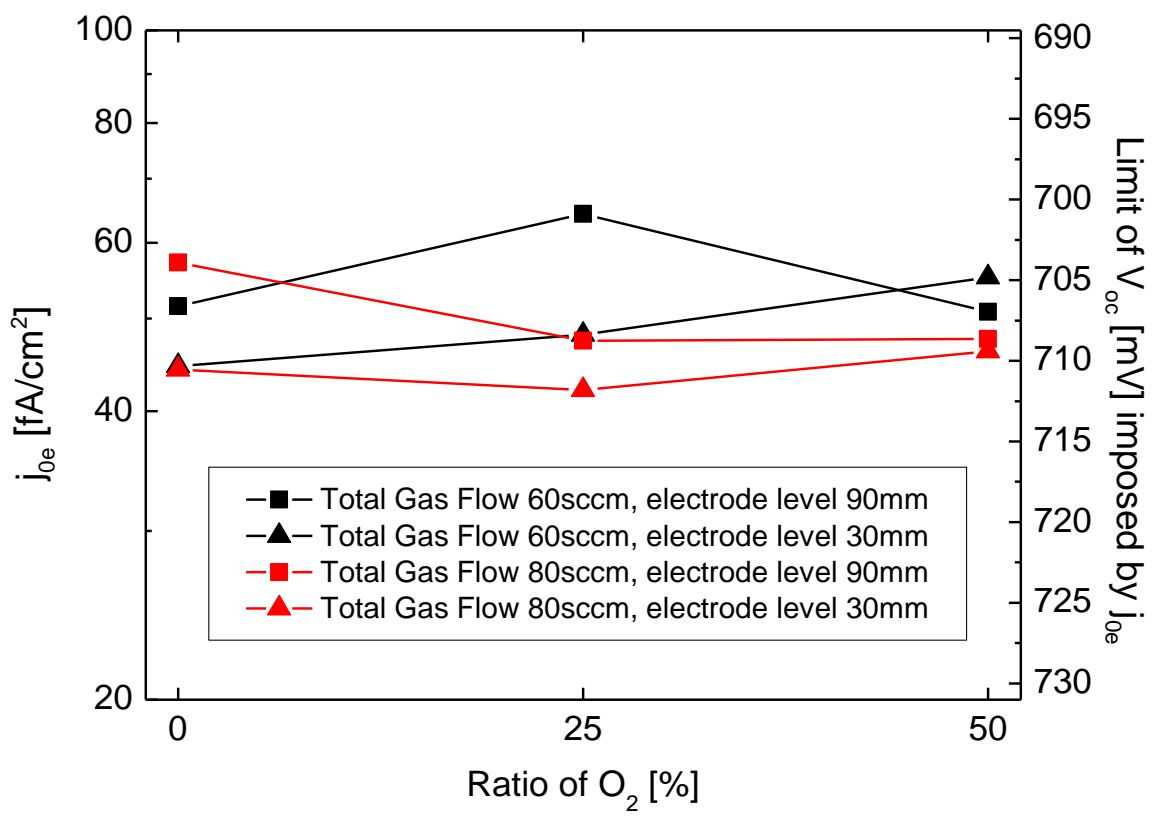

Figure 5.11: Influence of the ratio of $\mathrm{SF}_{6}$ and $\mathrm{O}_{2}$ as well as two different levels of the lower electrode in the RIE process on the emitter saturation current density $j_{o e}$ and the thereby limited implied open-circuit voltage $V_{\text {oc, implied }}$ (note the reversed scale).

These values are all in an excellent range with limits of $V_{o c}$ above $700 \mathrm{mV}$ revealing that mc-Si solar cells will not be limited in their efficiencies for any of these process variations. However, these values for maskless processing only show the pure influence of the etching processes and do not include any information on how a textured surface will limit $V_{o c}$. In this case, additional effects, e.g. the surface enlargement, will come into play. This study has shown that severe damages are not introduced by the RIE processing itself. What's more, after the RIE process, the isotropic MW plasma will remove potentially damaged areas.

Besides these electrical investigations on the RIE process, the optical quality of the resulting texture is defined already to some extent. Desired characteristics of the 
NIL and RIE generated etching pits concern mainly their profile shape as well as achievable aspect ratios. The assessment of the quality of the profile shape is mainly done considering the shape of the bottom of the etching pits as well as the shape of the sidewalls. For the bottom of the pits it is desired to have an ideally conic profile and unwanted to have a flat bottom. The latter typically will remain flat after the following isotropic etching. Figure 5.12 shows exemplary profile shapes with respect to the bottom of the etching pits.
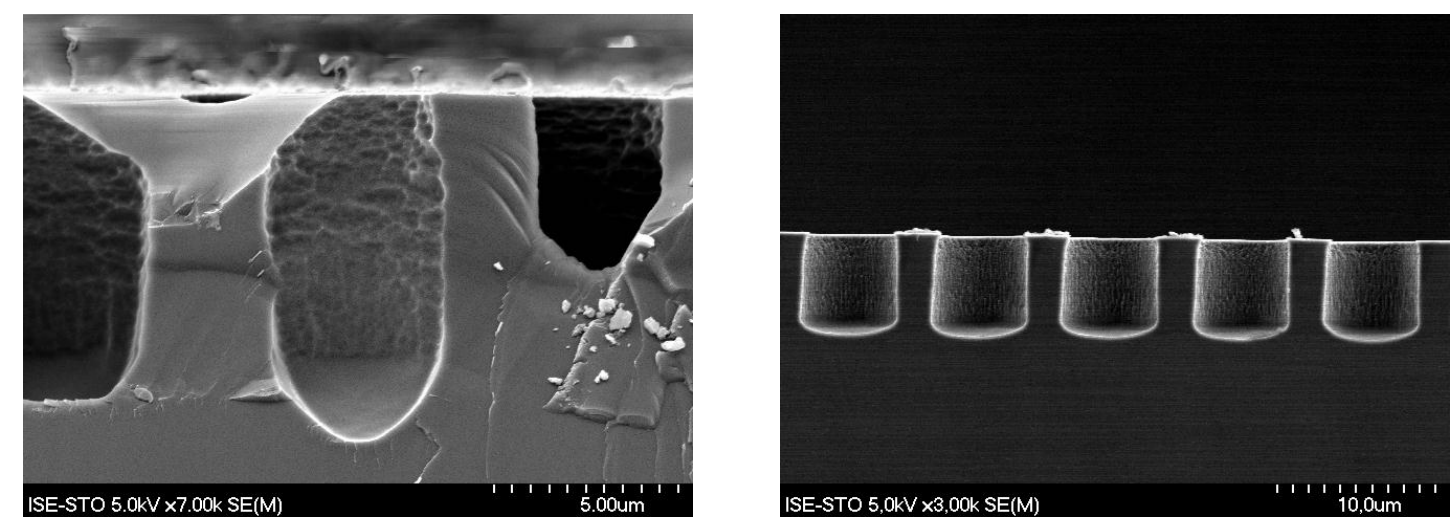

Figure 5.12: SEM micrographs of etching pit profiles after NIL, RIE and resist removal. The focus for these images concentrates on the bottom of the etching pits. Left: A nearly conic shape is realised. Right: A broad flat bottom in the etching pits is generated.

Besides the shape of the bottom, which can be manipulated by varying the RIE process parameters, the shape of the sidewalls as well as the aspect ratio will also have a significant influence on the achievable final texture after the following MW plasma process. These two parameters are linked to some extent. It was found that for very distinct processing conditions, strongly depending on the type of resist, very steep sidewalls and thus a very high aspect ratio of the etching pits can be achieved. One potential reason is that for these conditions an effective plasma polymerisation process occurs, which leads to a sidewall passivation of the etching process [189]. The origin of this polymerisation process lies in the (slow) decomposition of the resist in the plasma etching process. Typical resists mainly consist of hydrocarbon chains. These components, together with other species built from the etching gases (especially the fluorine) and the etched substrate, can polymerise again and be deposited as a thin layer on the substrate to be textured. The ion bombardment on the bottom of the etching pits opens this polymer layer locally, so that the depth of the etching pits can be increased. Chemically these fluorocarbon polymers are very stable and thus a purely chemical attack at the silicon sidewalls is inhibited.

Making use of this phenomenon, etching pits of high aspect ratios can be realised even for very low bias voltages and high chamber pressures $\left(\mathrm{V}_{\text {bias }}<40 \mathrm{~V}\right.$ and $\mathrm{p}=$ 
$20 \mathrm{~Pa}$ ). Figure 5.13 shows etched samples with a pronounced and a less effective sidewall passivation. The less effective sidewall passivation in this case was found for a sample that was masked only on a small area and the rest of the wafer's silicon surface was exposed to the plasma. An approach to explain this behaviour of a reduced anisotropy for this sample is that the larger amount of silicon being in interaction with the plasma gases leads to a varied plasma composition. This again might lead to a less effective polymer deposition.

a)

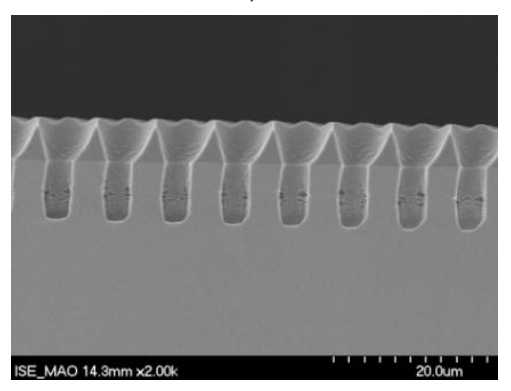

b)

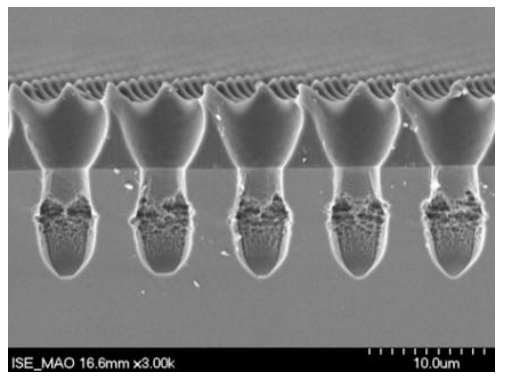

c)

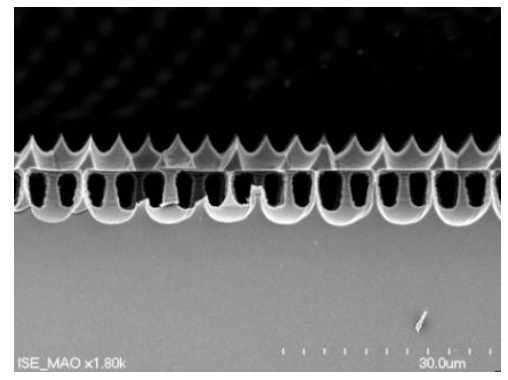

Figure 5.13: SEM micrographs of etching pit profiles after NIL and RIE visualising the effect of the fluorocarbon polymer sidewall passivation. All images are etched with the same processing parameters. For the images in the middle and on the right (right: longer etching time), there was additionally some unmasked bare silicon in the plasma chamber.

The formation of a thin flurocarbon polymer film via plasma deposition was further investigated. After RIE, an extensive plasma ashing process was conducted for several hours. In the SEM no resist residues were detected afterwards; however, a non-removable film at the sidewalls of the etching pits was left. Samples were characterised via Auger spectroscopy to obtain information about the material composition. By doing this in combination with a sputter etching of the analysed samples, this information can be obtained as function of the etching depth. On the one hand, the fluorocarbon polymer film is wanted to achieve high aspect ratios even for low bias voltages in the RIE process. On the other hand, it has to be removed afterwards, as it is a contamination of the wafer surface and can inhibit the following etching processes when it comes to a widening of the etching pits. Therefore, this investigation not only aims for the confirmation of the formation of this polymer layer, but also of the removability of it. In the literature it can be found that these fluorocarbon polymer films can be removed in aqueous hydrofluoric acid (HF) solutions [190].

Figure 5.14 shows the result of this investigation for a sample directly after the resist removal as well after an additional wet-chemical etching step in a $20 \%$ solution of HF. There, no silicon areas were exposed to the plasma on purpose (corresponding to the sample in Figure 5.13). No absolute values for the information of the depth can be given, since no calibrated measurement was 
available. However, since the samples are identical (except for the additional etching step), they can be compared with each other. It can be seen that the sample without the HF treatment shows a fluorine concentration of about 4 atomic per cent, which was not found after the etching step. Thus it can be concluded that both the formation of this film takes place and also the removal is conducted successfully. Furthermore, it can be seen that the HF treatment removes the native silicon oxide, which afterwards again is formed in a very shallow depth.
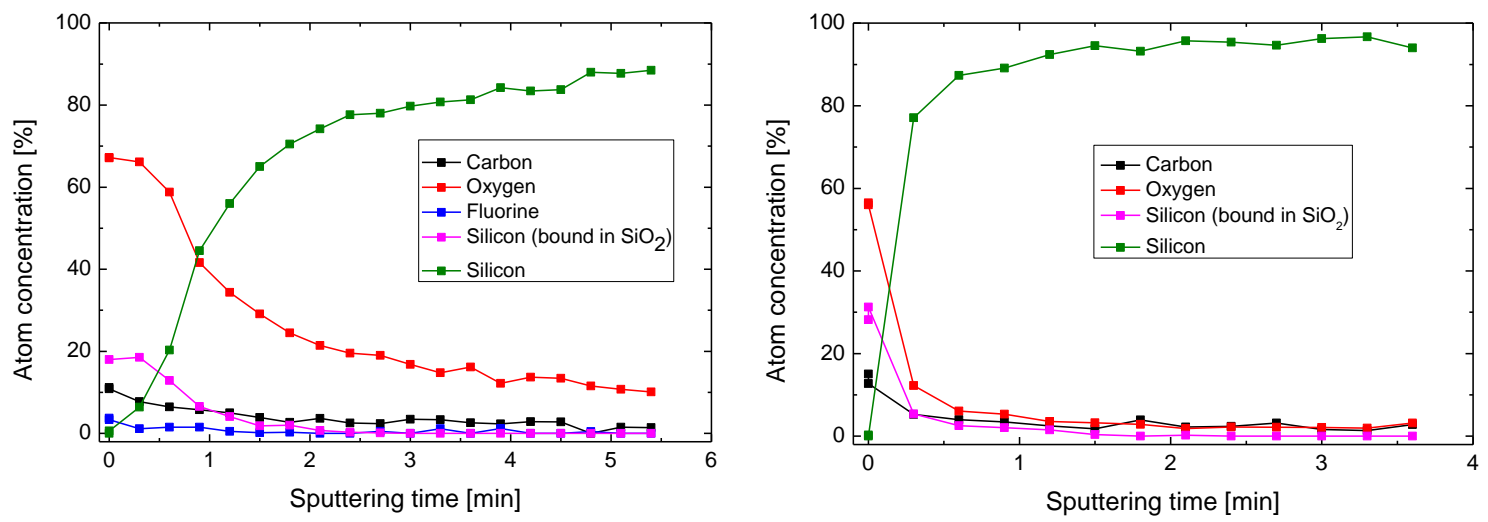

Figure 5.14: Material composition determined via Auger spectroscopy for different sample depths, which experienced a RIE process with a resist etching mask, a resist removal (left) and an additional etching step in hydrofluoric acid of $20 \%$ concentration for one minute. The measurement sequence switches between measurement of atomic concentrations and a sputter etching of the measured surface. Therefore, insight into the depth dependency of the concentrations can be gained. As the sputter etching process is not calibrated for silicon, the depth is solely displayed as a sputtering time.

Considering the demands set to achieve an optimum starting point for the isotropic etching process from the optics point of view, the following set of process parameters was found:

- gas flows: 45 sccm SF 6 ; 15 sccm O (Net gas flow: 60 sccm)

- RF-power: $300 \mathrm{~W}$

- pressure: $20 \mathrm{~Pa}$

- substrate temperature: $-15^{\circ} \mathrm{C}$

- electrode level: $90 \mathrm{~mm}$

This corresponds to the process for which the maximum etching rate was determined in the beginning. However, the lowest values of implied $V_{o c}$ were also found for this process. Still, this process was chosen to optimise the following MW plasma etching process. The reason for this decision is that the overall damage by the RIE process was found to be marginal and an etching step follows, in which this slightly degraded material can be removed. 


\section{Resist removal}

The removal of crosslinked negative tone resists is not a trivial task. Some strategies or processess were already explained concerning their basic mechanisms in section 4.3.3. In the following, some options for a resist removal after the RIE process are described. These options can be first divided into the groups of wet and dry-chemical removal. For the wet-chemical approach, there are the options of etching the resist or dissolving a sacrificial layer beneath the resist. The drychemical route investigated within this work is based on optimised plasma ashing processes. In Figure 5.15, these options are summarised and materials or processing details are introduced. The investigations regarding the resist removal were conducted using the Roller-NIL tool and Laromer P084F as the resist if not declared otherwise.

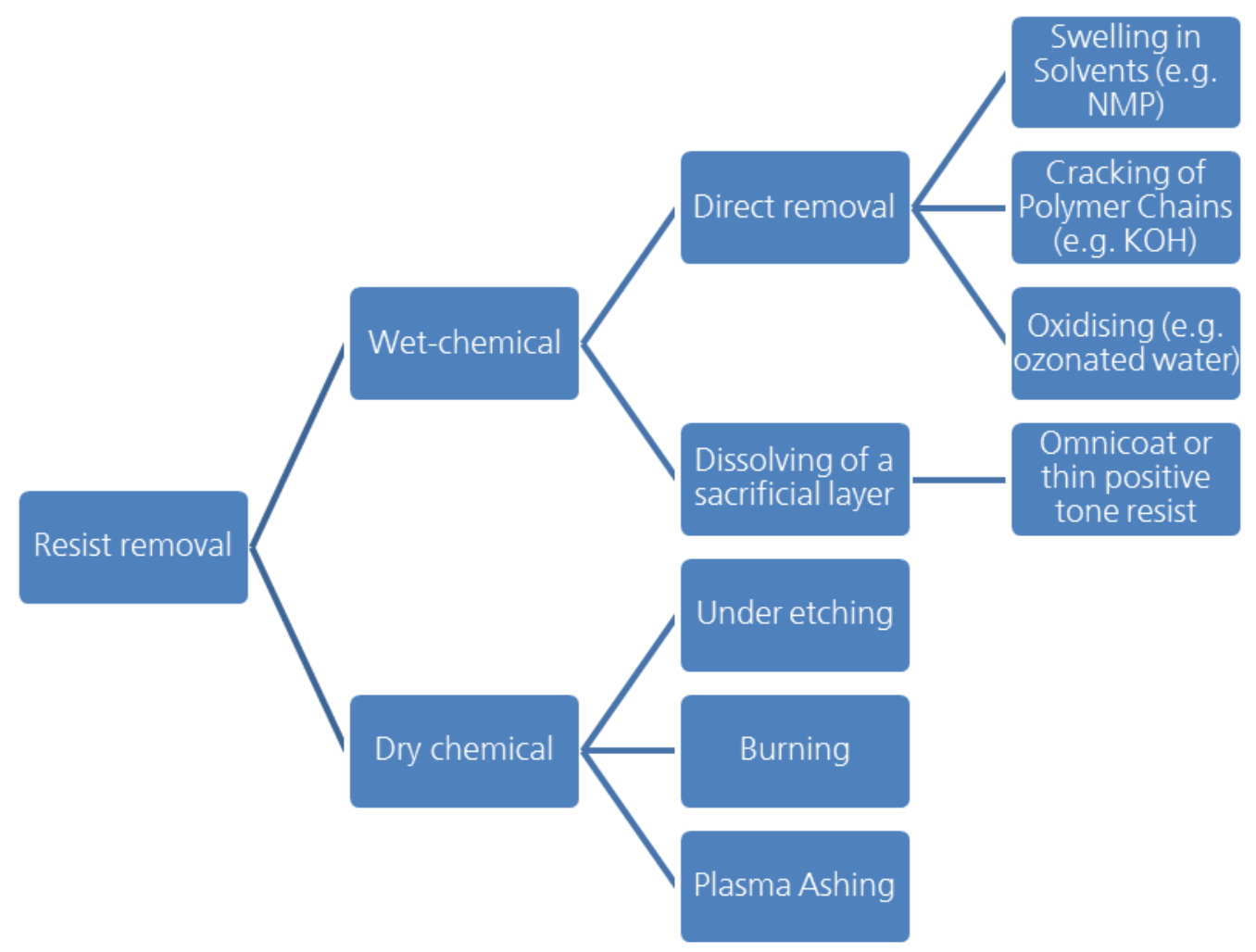

Figure 5.15: Overview of possible technological routes for the removal of resist residues after reactive ion etching.

\section{Wet-chemical resist removal}

First, experiments were conducted where the resist mask was under-etched completely and afterwards could be blown away with a nitrogen pistol. However, this process depends crucially on a very high homogeneity of the imprinted resist layer and the etching rates in the plasma etching process. 
In some datasheets of crosslinking resist materials, it can be found that a swelling in organic solvents is a suitable measure to remove these resists fully. It was found that this holds for the solely imprinted and cured resist masks; however, after the RIE etching with related temperature treatment and exposure to harsh UV-radiation, this is not possible anymore.

A promising approach is based on the application of a sacrificial layer beneath the actual resist. One possible solution, which was investigated within this work, is the product Omnicoat from MicroChem ${ }^{\mathrm{TM}}$. This material is suggested as an adhesion promoter as well as sacrificial layer for SU8 in LIGA ${ }^{14}$ process applications. This material is spin-coated prior to the resist coating as was introduced in the introduction of the NIL process in this chapter. After the spin coating process, a baking process follows during which all the solvent is driven out. The processing of this material was done according to the datasheet. After the NIL and the RIE process the Omnicoat layer is dissolved in the organic solvent N-Methyl-2pyrrolidone (NMP) at $80^{\circ} \mathrm{C}$. Figure 5.16 shows a SEM micrograph of c-Si as well as a photograph of a mc-Si substrate after NIL, RIE and resist stripping via dissolving of the Omnicoat layer (right). The c-Si sample was processed using the conventional NIL tool and SU8-2002 as resist.
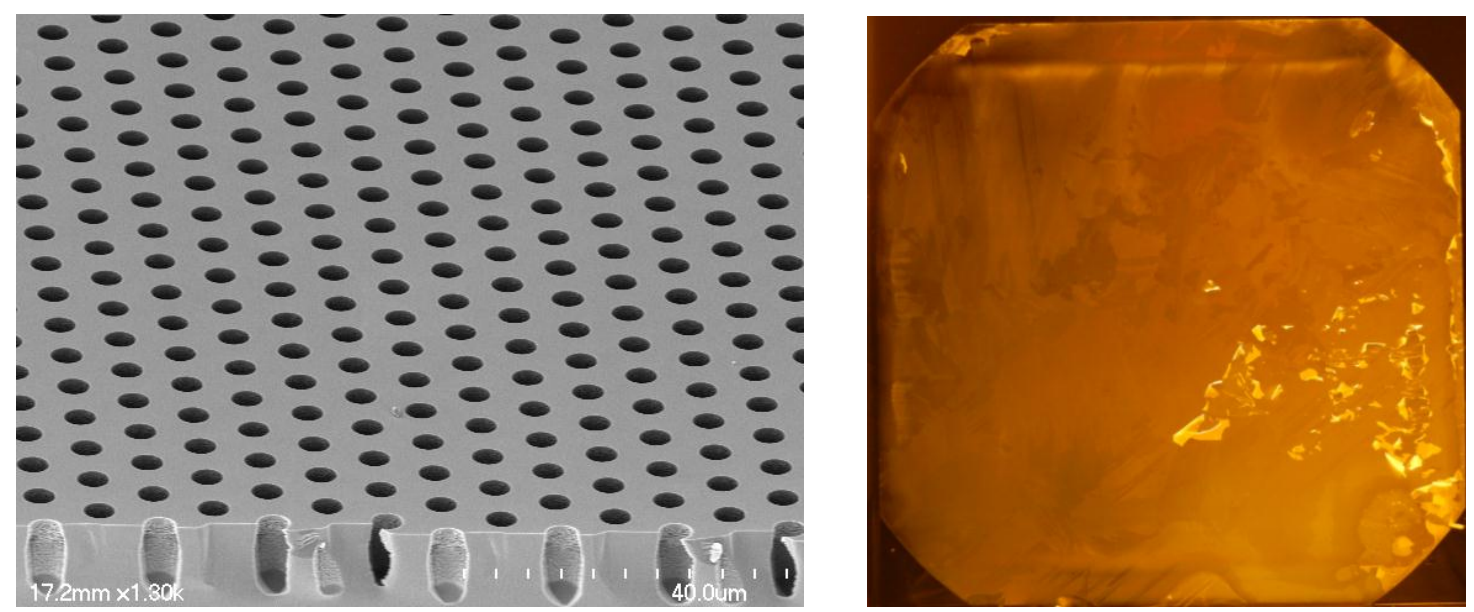

Figure 5.16: Silicon substrates after NIL, RIE and resist removal via dissolving of a sacrificial layer. Left: SEM micrograph of a successful removal of the etching mask on a bright etched monocrystalline silicon substrate. Right: Photograph of a pseudo-square $156 \times 156 \mathrm{~mm}^{2}$ substrate showing that there are still resist residues left after the removal process. There, the multicrystalline silicon surface was chemically polished prior to the texturing process chain.

The difference in the resists applied for the samples shown in Figure 5.16 was of no relevance. It was found that this process works on bright etched surfaces;

14 LIGA process: The German acronym LIGA stands for a process sequence of Lithography, Electroplating and embossing for the fabrication of high aspect ratio features. 
however, even for chemically polished mc-Si substrates, a complete removal over the whole surface was not achieved. Nevertheless, this process chain was applied for the processing of bright etched c-Si substrates. It is assumed that the surface roughness of the substrates is the most relevant parameter influencing the resist removal. The Omnicoat layer thickness was determined to be about $13 \mathrm{~nm}$ using ellipsometry. This very thin layer and the fact that the material Omnicoat consists almost entirely of solvent and has a very low viscosity gives rise to the suspicion that an incomplete coverage of the Omnicoat might be the source of this problem. However, several attempts using modified spin coating parameters or even multiple spin coating processes did not lead to a reproducible resist removal.

Therefore, another material, which can be applied as thicker layer, was investigated for use as a sacrificial layer. For this purpose, the positive tone

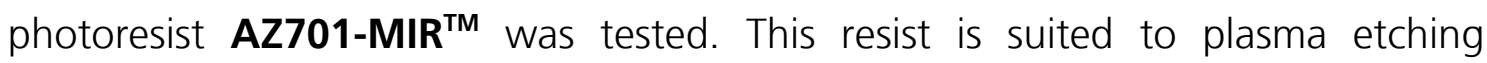
processes and, therefore, has a high thermal stability. Furthermore, contrary to negative tone resists, a dissolving of this resist is possible. This resist was applied in various thicknesses up to $400 \mathrm{~nm}$ and the resist removal after RIE was investigated for several grades of surface quality of silicon wafers from bright etched to mc-Si as-cut surfaces. It was found that a complete resist removal can be achieved even for very rough surfaces, when using $400 \mathrm{~nm}$ thick AZ701-MIR ${ }^{\mathrm{TM}}$ layers. However, it was also found that the homogeneity of the resulting texture suffers strongly. The reason therefore is that the spin coating of the AZ701-MIR ${ }^{\mathrm{TM}}$ layer leads to a levelling, on which the NIL resist is then spin-coated. As the AZ701-MIRTM also acts as an etching resist, the inhomogeneity in this layer leads to an inhomogeneous overall residual layer thickness beneath the imprinted pattern. Therefore, a strongly varying etching depth is obtained after the RIE process (see Figure 5.17).
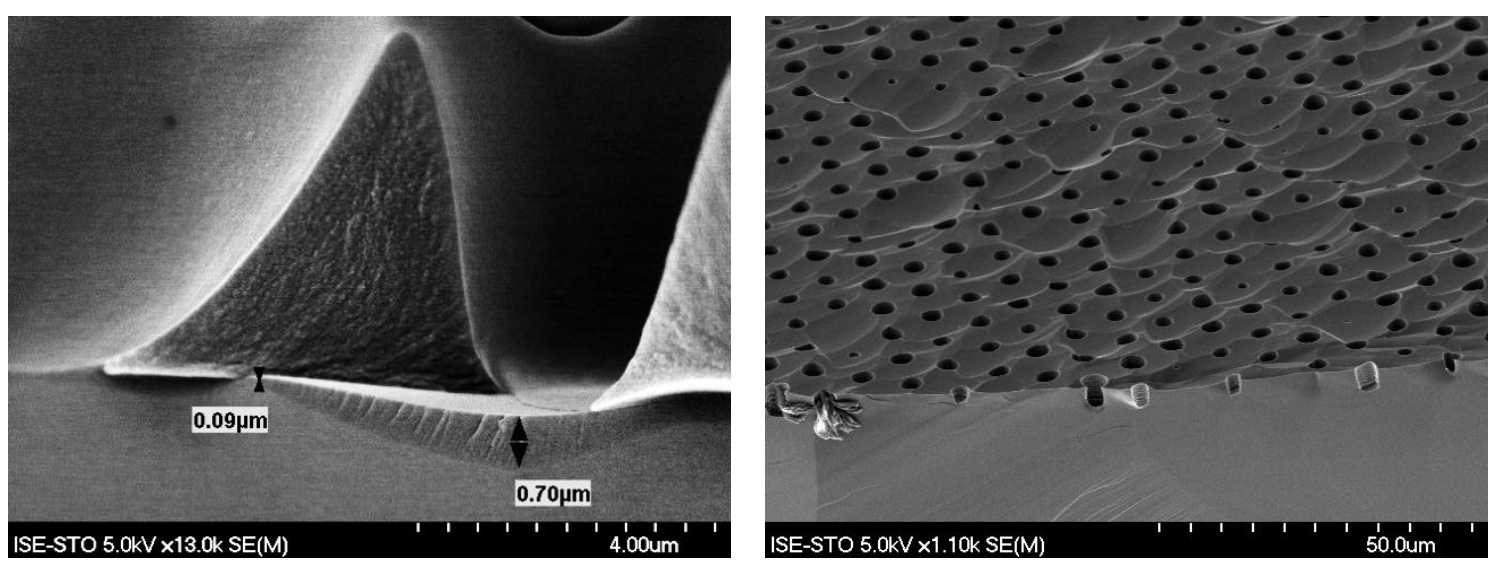

Figure 5.17: SEM micrographs: Left: Imprinted resist layer on top of a nominal $400 \mathrm{~nm}$ thick positive tone resist layer (as sacrificial layer) on a rough mc-Si substrate. Right: Inhomogeneous texture resulting of a rough substrate and a thick sacrificial layer. 
In order to avoid these problems of inhomogeneity or incomplete resist removal related to the use of spin-coated sacrificial layers, other direct removal techniques were investigated. Some crosslinked resist materials can be removed in heated and strongly diluted alkaline solutions. It was shown that a complete resist removal after NIL and RIE in an aqueous $\mathrm{KOH}$ solution with a low $\mathrm{KOH}$ concentration of 0.5 per cent by weight at $80^{\circ} \mathrm{C}$ requires a treatment of at least seven minutes [191]. Even for this strongly diluted solution, a silicon removal was detected. More critical than the removal itself in this case is the anisotropic etching behaviour using alkaline solutions. As result of this, a crystal orientation-dependent modification of the etching pits occurs (see Figure 5.18). This makes this removal technique unsuitable for the given application on mc-Si substrates, which have different crystal orientations across one substrate. Increasing the concentration to 3 percent by weight leads to a faster removal; however, the etching of the silicon is also increased leading to worse results.
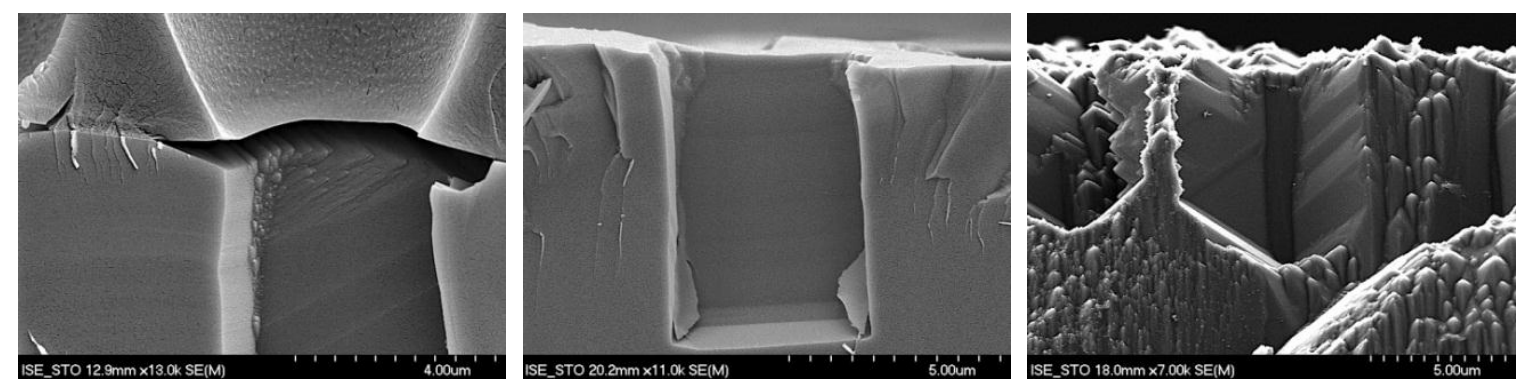

Figure 5.18: SEM micrographs showing the effect of the resist removal in different aqueous alkaline solutions at $80^{\circ} \mathrm{C}$ on mc-Si substrates. Left: Start of the resist removal after 2 minutes in $0.5 \%_{\text {weight }}$ $\mathrm{KOH}$ solution. Middle: Sample with removed resist after 7 minutes in $0.5 \%_{\text {weight }} \mathrm{KOH}$ solution. The bottom of this etching pit is completely planarised. Right: Sample with removed resist after 1 minute in $3 \%_{\text {weight }} \mathrm{KOH}$ solution.

\section{Dry chemical resist}

Plasma ashing is a well-known technique to remove polymeric resists. However, for pure oxygen plasma ashing, the etching rates in crosslinked resist systems are typically very low. Dentinger et al. [157] and Engelke et al. [159] demonstrated increasing etching rates of crosslinked SU8 resist by adding tetrafluoromethane $\left(\mathrm{CF}_{4}\right)$ to the oxygen. There, it was shown that by adding a $\mathrm{CF}_{4}$ gas flow in the range of less than $10 \%$ of the total gas flow, resist etching rates above $1 \mu \mathrm{m}$ per minute can be achieved for SU8. A design of experiments (DoE) was set up to investigate achievable resist etching rates for Laromer PO84F [191]. Without $\mathrm{CF}_{4}$ only very small abrasion rates of the resist of less than $200 \mathrm{~nm}$ per minute were found. As a result of the DoE, a maximum resist etching rate of above $2 \mu \mathrm{m}$ per minute is predicted for the following set of parameters: 
- gas flows: $90 \mathrm{sccm} \mathrm{O}_{2} ; 5 \mathrm{sccm} \mathrm{CF}_{4}$

- pressure: $17 \mathrm{~Pa}$

- level of the electrode: $120 \mathrm{~mm}$

- power: 50 W RF-power; 1000 W MW-power

- temperature: $-15^{\circ} \mathrm{C}$

The substrate temperature was not increased in this study; however, it can be expected that this should further increase the resist etching rate. A verification of the efficiency of this prediction was also demonstrated in [191]. Furthermore, an indicator was found to determine the progress of the resist removal during the resist ashing. The bias-voltage was found to drop at the time of the complete removal (see Figure 5.19). This could easily be implemented as a process control. It is also shown that for different substrate sizes and slightly different processing conditions the absolute level as well as the time of the drop changes. Still a very characteristic drop can be found. Furthermore, the drop in the bias-voltage after around three minutes of processing confirms the high resist etching rates of about $2 \mu \mathrm{m}$ per minute, as the patterned resist layer after NIL and RIE is still up to $7 \mu \mathrm{m}$ thick. What is also important is that although $\mathrm{CF}_{4}$ was added as processing gas, no significant etching of the silicon substrate was detected for the investigated processes.

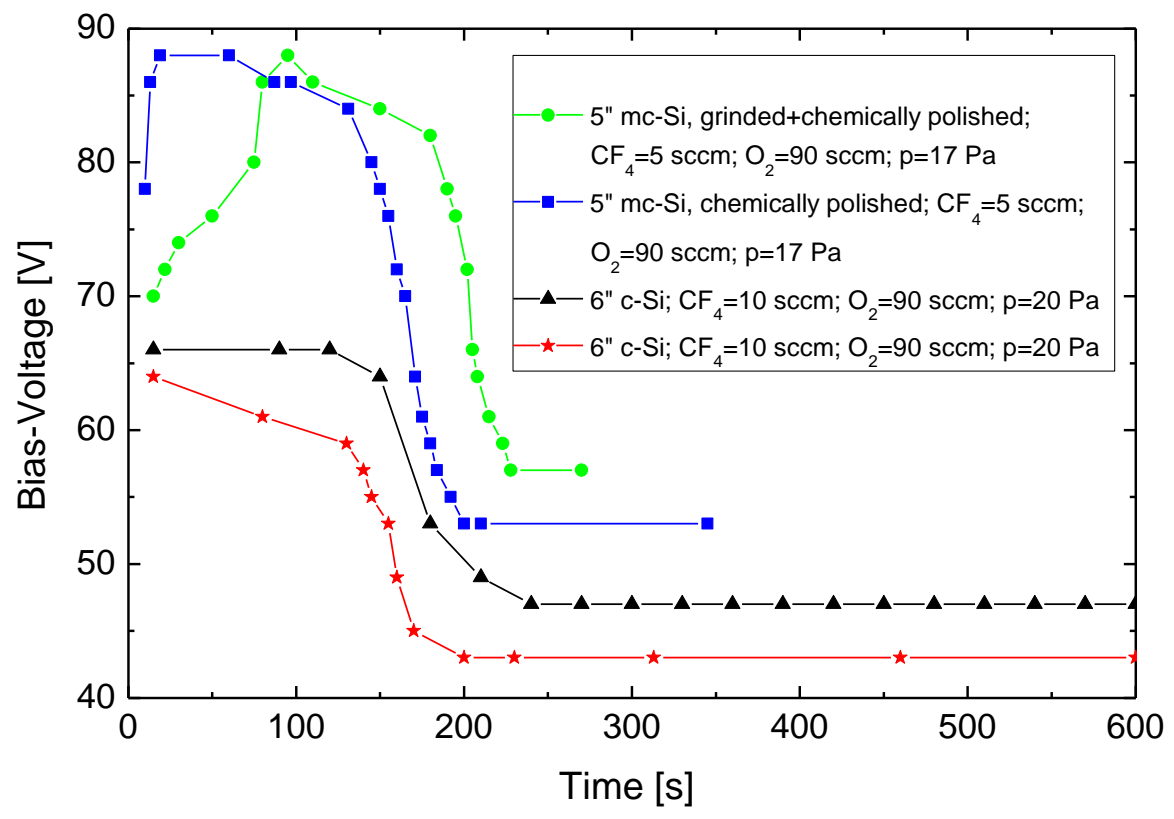

Figure 5.19: The bias-voltage plotted against processing time during the plasma ashing. Shown are results for different substrate sizes and pre-conditionings.

When controlling the completeness of the resist removal in the SEM, residues were found. These residues could not be removed even for a very long plasma ashing 
process. Therefore, it was concluded that these residues have to be inorganic and cannot be removed in oxygen plasmas. Presumably, these inorganic components are originally bound in the photo initiator as is known e.g. for salts in SU8 [192]. These residues were characterized using energy-dispersive $X$-ray spectroscopy (EDX) and inductively coupled plasma optical emission spectrometry (ICP-OES). This way it could be excluded that these inorganic residues are of metallic nature. Furthermore, an effective removal of these residues in hydrofluoric acid is possible as shown in Figure 5.20. In the micrographs after the removal of these residues, it can be seen that there is a kind of porous layer left in the areas where the residues have been. The origin of this porous layer was not identified within this thesis.

a)

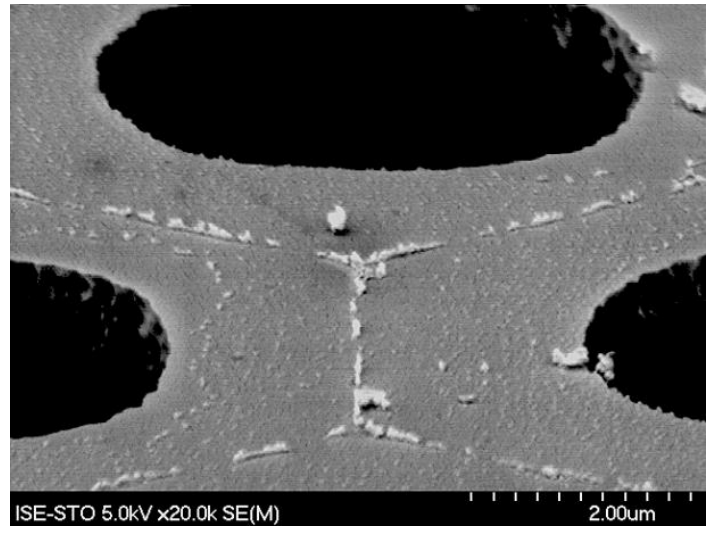

c)

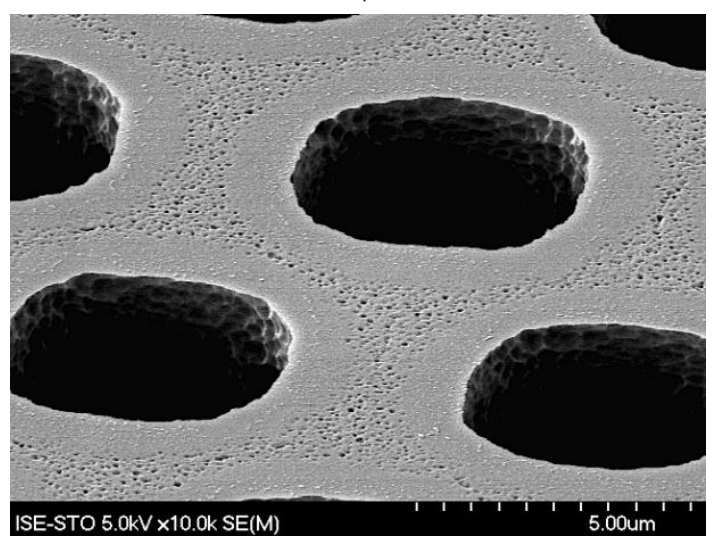

b)

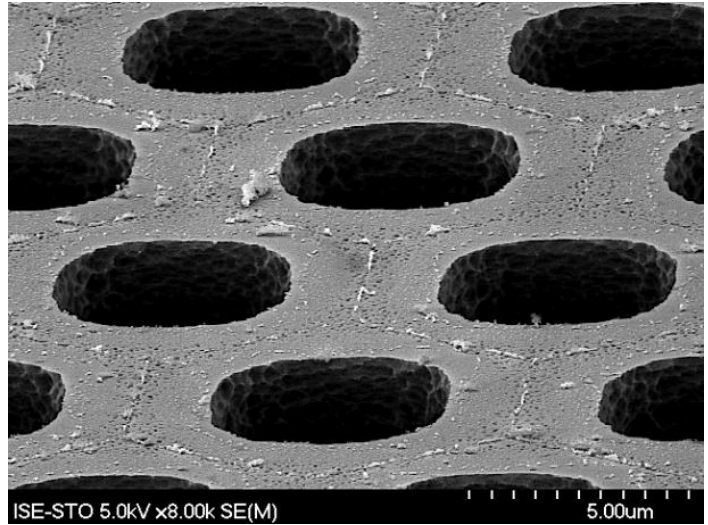

d)

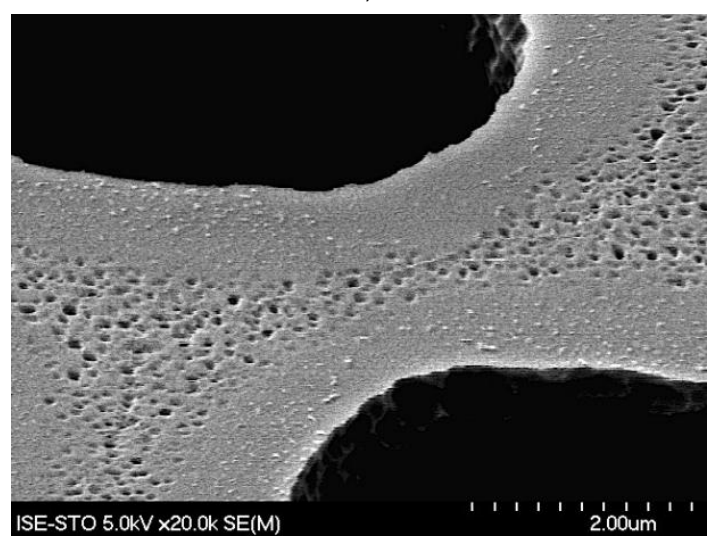

Figure 5.20: SEM micrographs of silicon surfaces after NIL, RIE and plasma ashing. The images a) and b) show that for increasing plasma ashing times no removal of the residues occurs (10 and 15 minutes respectively). The lower two images c) and d) show, that a removal of these residues in hydrofluoric acid is possible.

\section{Rating of the presented methods for resist removal}

In the presented process chain, based on plasma etching processes, a dry chemical resist removal might be advantageous compared to wet-chemical approaches. 
However, also for the dry chemical removal, a wet-chemical HF treatment was found to be necessary to fully remove residues. Whether this step is necessary to achieve good solar cell performance was not investigated within this work.

As an alternative route, it would also be possible to set up a combination of dry and wet-chemical etching processes. The advantage of RIE processes could be used to generate deep etching pits and the isotropic widening of the etching pits could be carried out wet-chemically. Then a wet-chemical resist removal could be easily integrated into the process chain. Table 5.2 summarises some characteristics as well as the advantages and disadvantages of the presented resist removal techniques.

Table 5.2: Summary and rating of the presented resist removal techniques.

+ = good; 0 = moderate; - = bad.

\begin{tabular}{l|c|c|c|c|c}
\hline Technique & $\begin{array}{c}\text { Complexity / } \\
\text { Process steps }\end{array}$ & Effectiveness & $\begin{array}{c}\text { Requirements } \\
\text { on } \\
\text { homogeneity/ } \\
\text { process window }\end{array}$ & $\begin{array}{c}\text { Integration in } \\
\text { the process } \\
\text { chain }\end{array}$ & $\begin{array}{c}\text { Effect on } \\
\text { texture } \\
\text { quality }\end{array}$ \\
\hline Swelling & + & - & 0 & 0 & + \\
\hline Sacrificial layer & $-(+1$ step) & $\begin{array}{c}\text { surfaces) } \\
\text { - (rough } \\
\text { surfaces) }\end{array}$ & - & $\begin{array}{c}\text { - (for dry } \\
\text { processing) } \\
+ \text { (for wet } \\
\text { etching) }\end{array}$ & $\begin{array}{c}\text { - (for } \\
\text { thick } \\
\text { layers }\end{array}$ \\
\hline KOH etching & + & 0 & $\begin{array}{c}- \text { (for dry } \\
\text { processing) } \\
+ \text { (for wet } \\
\text { etching) }\end{array}$ & - \\
\hline Underetching & + & 0 & - & + & - \\
\hline Plasma Ashing & + & + & + & + & + \\
\hline \hline
\end{tabular}

\section{Microwave (MW) plasma etching}

The purpose of this second etching process is to widen the etching pits isotropically. Additionally, this etching process shall be as soft as possible so as to not introduce any plasma damages and to allow low surface recombination velocities of the textured substrates. For these reasons a MW plasma is applied in which the ions are not accelerated onto the substrate to avoid a bombardment. Thereby, a purely chemical etching behaviour can be achieved. The process applied for this purpose was developed by S. Schäfer and is described in [78]. There, it is shown that for MW plasma etching of silicon, etching rates above $500 \mathrm{~nm}$ per minute can be achieved (see Figure 5.21). The etching rate increases for pressures 
smaller than $8 \mathrm{~Pa}$. These high etching rates are related to very high plasma densities as can be achieved for electron-cyclotron-resonance (ECR) conditions (see also section 4.4). A higher plasma density in this case is related to a higher concentration of reactive species, which again leads to higher etching rates. In the study of Schäfer, solely $\mathrm{SF}_{6}$ was used as etching gas. In the present work, argon was additionally introduced into the chamber. The introduction of argon when etching silicon in RIE processes has the benefit of introducing additional physical sputtering processes and thus removing potentially built polymer films. When applying purely isotropic etching processes, the radical density can be influenced by the addition of argon [114].

Furthermore, in [78] effective minority carrier lifetimes were also determined for different pressures in the MW plasma etching process. As for the etching rate, the optimum lifetime values of above $900 \mu$ s were achieved for a pressure of $1 \mathrm{~Pa}$. Therefore, this process was chosen for the widening of the etching pits after NIL, RIE and resist removal within this work.

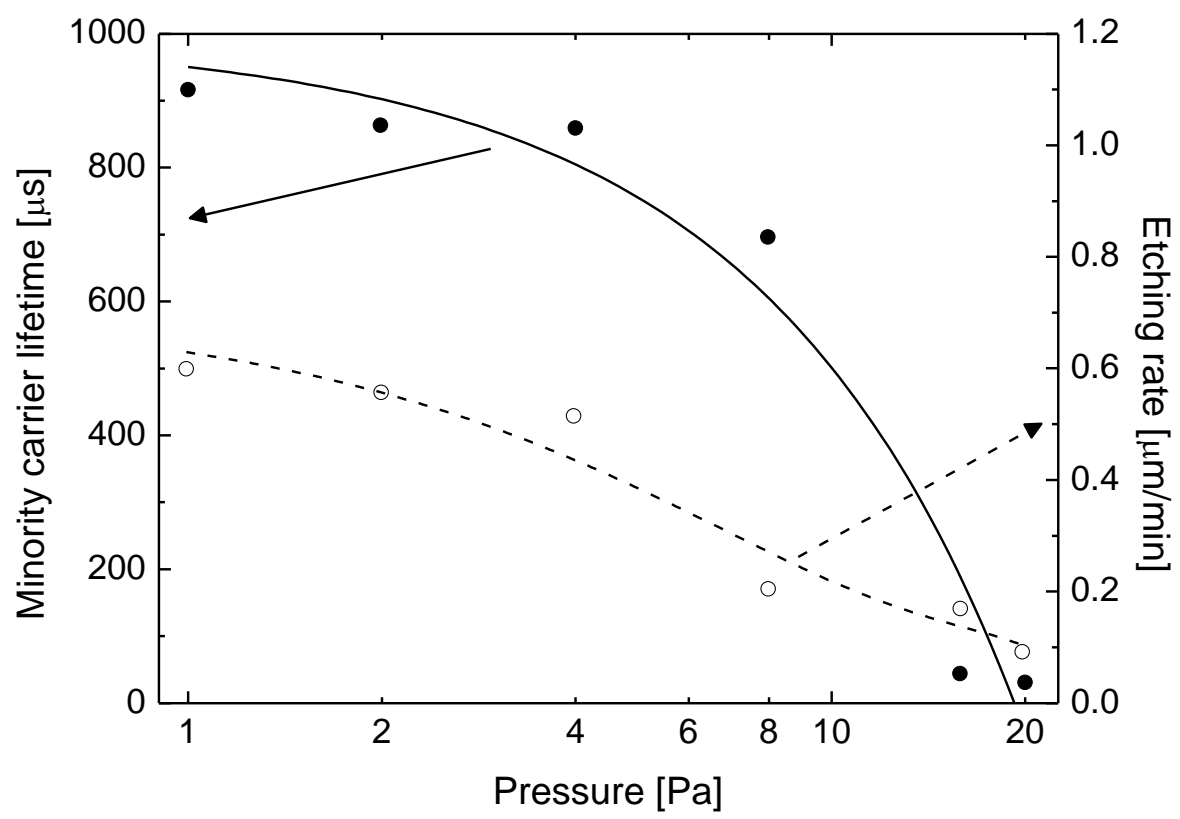

Figure 5.21: Effect of the pressure during MW plasma etching of silicon using $\mathrm{SF}_{6}$ on the etching rate as well as the effective minority carrier lifetime determined for $F Z p$-type silicon passivated by a PECVD silicon nitride. Lines are guides to the eyes. The data is taken from [78].

Applying this isotropic etching process to the RIE pre-patterned substrates, the optimum etching time has to be evaluated. Figure 5.22 shows c-Si as well as mc-Si substrates after the MW-plasma etching process in cross-section as well as tilted topview. For these samples, SU8 was patterned in the conventional NIL tool and the resist removal was conducted using Omnicoat as a sacrificial layer. The process optimisation for RIE and MW plasma etching was mainly conducted using this 
resist. However, the behaviour of the etching processes using Laromer P084F patterned via Roller-NIL required only minor changes in the processing parameters. The main parameter that was changed for this resist was the etching times. This could be related to a more pronounced deposition process for this resist.
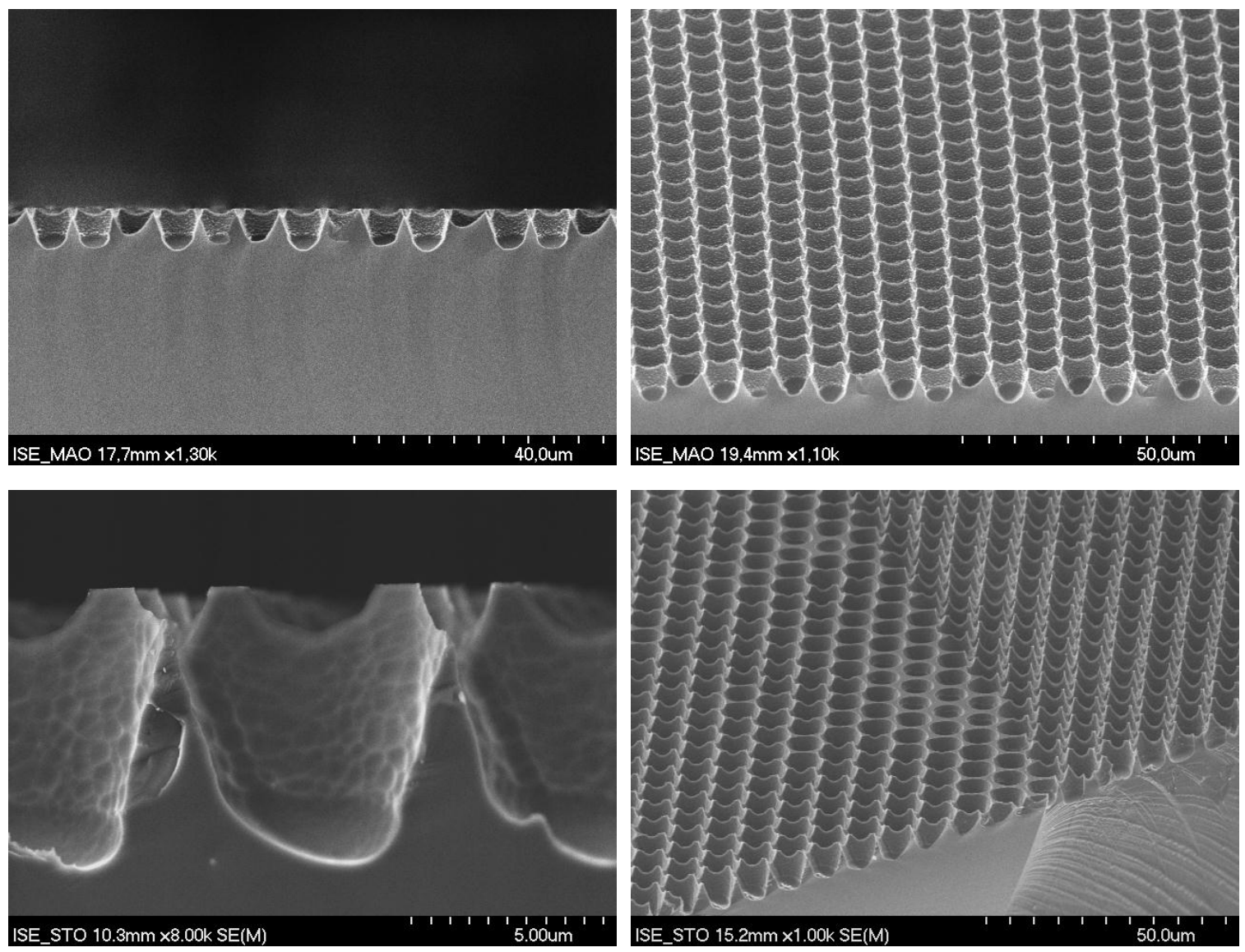

Figure 5.22: SEM micrographs of honeycomb textured silicon surfaces after NIL, RIE, resist removal and MW plasma etching. The upper two images show the resulting texture on monocrystalline silicon, the lower two on multicrystalline silicon.

\subsubsection{Optical and electrical characterization}

In Figure 5.22 it can be seen that the honeycomb texture on c-Si has a profile shape very similar to the target one formulated in subsection 5.1.1, where the optimal profile shape was defined:

- The aspect ratio is about one,

- the etching pits merged, so that there are no planar areas in between,

- the bottom of the etching pits seems to be non-planar,

- in the cross-section view a kind of conic shape is approximated.

On mc-Si, a slight distortion of the pattern can be seen in the cross-section. This might be related to a stamp deformation during the NIL process due to the uneven 
surface. In the SEM micrograph of the tilted top view, the influence of a step at a grain boundary on the resulting texture can be seen. Directly at the grain boundary in this case probably the residual layer thickness was too high after NIL. This led to a smaller etching depth and diameter after RIE and, therefore, the etching pits are not merged after the MW plasma etching process. However, it can also be seen that this negative effect occurs only locally.

The optical quality of these honeycomb textured substrates is determined by reflection measurements. These measurements were conducted using a Fourier spectrometer. Three reference systems were chosen to assess the quality of the fabricated textures as shown in Figure 5.23. These were a planar reference, an isotextured mc-Si substrate and a c-Si substrate with inverted pyramids [193]. All samples were measured without anti-reflection coating. To be better able to compare the reflection measurements, values for the weighted reflection $R_{w}$ as defined in equation (3.1) are given in the legend in Figure 5.23.

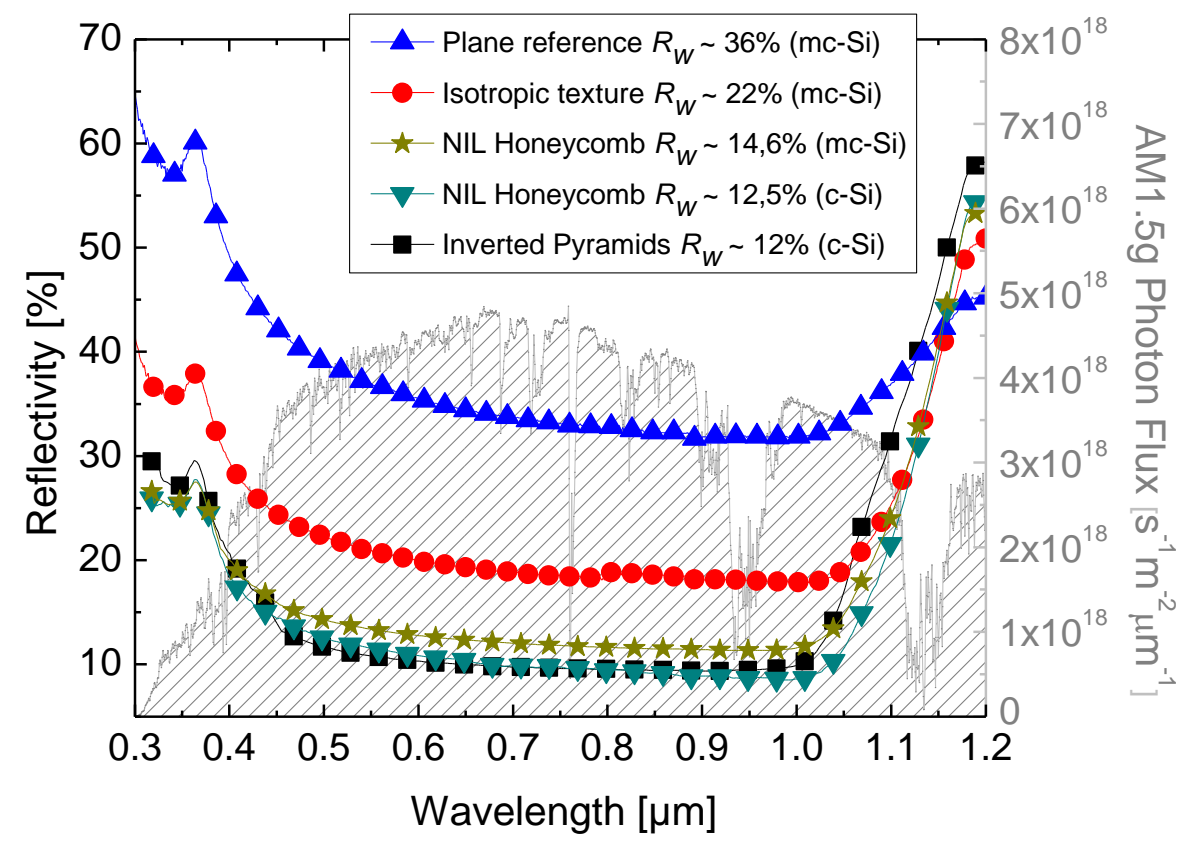

Figure 5.23: Reflection measurements of differently textured substrates without antireflection coating. In the background the photon flux of the solar AM1.5g spectrum is shown. The reflectivity was averaged over the wavelength range using the AM1.5g spectrum as weighting. The average values are shown in the figure legend for each [193].

When comparing these measurements, care has to be taken for wavelengths above $1000 \mathrm{~nm}$ for which strong deviations might arise due to different topologies on the backside (e.g. for the isotextured substrate the backside has the same texture as the frontside). Also, the wafer thickness is important in this regime. The c-Si wafers shown in this graph are $250 \mu \mathrm{m}$ and the mc-Si wafer below $200 \mu \mathrm{m}$ 
thick. However, both points (rear side topography and thickness) are only crucial for these long wavelengths since the penetration depth for shorter wavelengths is smaller than the wafer thickness.

The isotexture is the state-of-the-art of industrial processing for mc-Si. Thus we have to compare the result of our process chain to this type of texture. The inverted pyramids texture on c-Si is a defined pyramidal texture for high-efficiency cells produced on a laboratory scale using photolithography and alkaline etching. There is a slight difference between the quality of the honeycomb texture on c-Si and on mc-Si. As mentioned before, it is supposed that this is a result of the residual layer thickness being slightly less homogeneous on mc-Si. However, on mc-Si, the optical quality of the honeycomb texture via NIL and plasma etching is considerably superior to the isotexture on $\mathrm{mc}-\mathrm{Si}$ and is even close to pyramidal textures realised on c-Si. On c-Si, the optical quality of the honeycomb texture is very close to that of the inverted pyramids texture, which is at present used for reaching the highest efficiencies for silicon solar cells.

Besides the optical characterisation, also the electrical properties of substrates textured with the presented process chain have to be investigated. Lifetime samples of different texturing schemes were prepared to evaluate surface recombination effects of the honeycomb texture. The different types of surfaces that were investigated are a random pyramids texture, an isotexture and the honeycomb texture fabricated by the presented process chain. The samples for the random pyramids and the honeycomb texture were processed on FZ $p$-type $8 \Omega \mathrm{cm}$ substrates. For a reasonable acidic wet-chemical texturing (comparable to the standard isotexture on mc-Si), it is necessary to use substrates still comprising saw damaged areas [71]. Because of a lack of FZ substrates of the same resistivity that do not yet have the saw damage removed, for the isotextured substrates FZ $p$ type $0.9 \Omega \mathrm{cm}$ material was used.

After the texturing of the substrates, a shallow $120 \Omega / \square$ emitter was realized by a $\mathrm{POCl}_{3}$ diffusion. On the front side a combination of a thin thermal oxide and an antireflection silicon nitride was applied. The backside was passivated using silicon nitride. The lifetime samples were then measured with the QssPC method. The isotextured substrate was evaluated at low level injection and the random pyramid and honeycomb textured substrates were evaluated at high level injection using the slope method [194], [195] as described in [196]. Figure 5.24 shows a graph with the calculated $j_{0 e}$ and thus implied $V_{o c}$ values. 


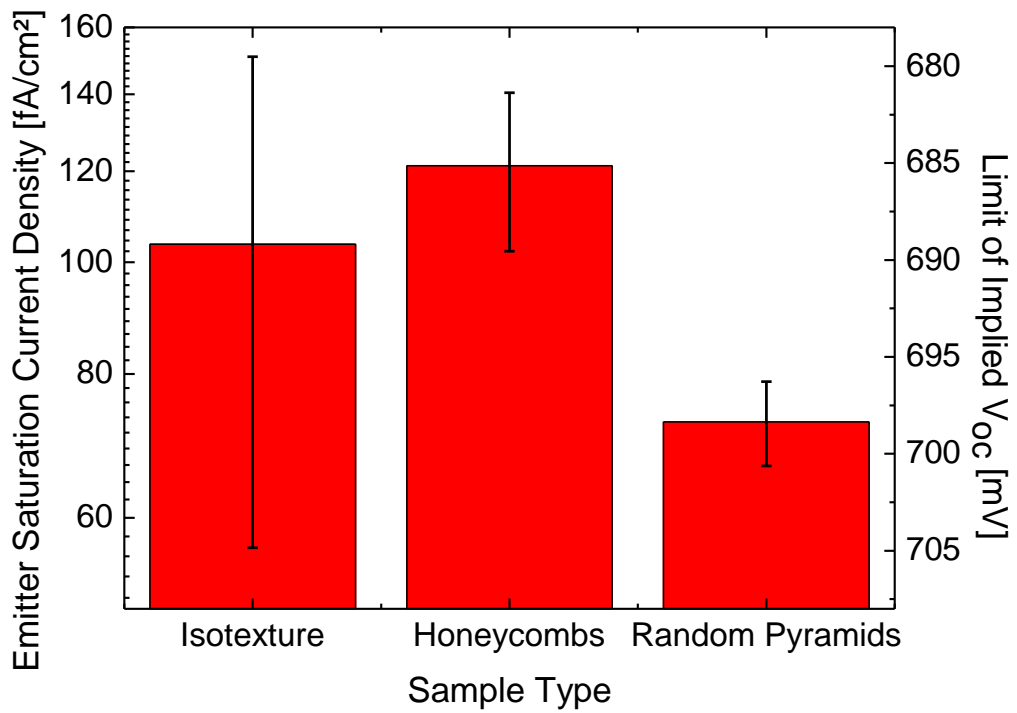

Figure 5.24: Values of the emitter saturation current density and the limit of the thereby implied $V_{O C}$ for substrates with different textures extracted from lifetime measurements using the QSSPC method.

The values for $j_{0 e}$ are slightly higher for the honeycomb texture compared to the isotexture $\left(121 \mathrm{fA} / \mathrm{cm}^{2}\right.$ and $103 \mathrm{fA} / \mathrm{cm}^{2}$ respectively). The random pyramids show values of around $73 \mathrm{fA} / \mathrm{cm}^{2}$. The values for the isotexture showed a considerable deviation. This was not investigated in more detail. Due to the rather distinct surface enlargement for the random pyramids and the honeycomb texture compared to a planar surface, one can put the two values of $j_{0 e}$ of these textures into relation. The random pyramids should have a surface enlargement of around 1.7 and the honeycombs of around 2.5 compared to a flat surface. This difference could already be used to explain the difference in $j_{0 e}$ as this quantity depends on the surface area. These measurements indicate that there is no major problem concerning contaminations or surface damages due to this new process chain.

\subsubsection{Solar cell processing}

This part is divided into a proof-of-concept stage, where small-area solar cells are fabricated using the conventional NIL tool, and an up-scaling part, where the Roller-NIL tool is used to texture large area silicon substrates.

\section{Fabrication of honeycomb textured solar cells using the conventional NIL tool}

The samples used for the determination of the electrical properties of honeycomb and isotextured samples were used to fabricate solar cells. Again, for the 
honeycomb textured substrates FZ $p$-type $8 \Omega \mathrm{cm}$ and for the isotextured substrates $0.9 \Omega \mathrm{cm}$ material was used (see also section 5.1.5). A shallow $120 \Omega / \square$ emitter was realized by a $\mathrm{POCl}_{3}$ diffusion. Again, the front surface was passivated by a $10 \mathrm{~nm}$ thick thermal silicon oxide with an antireflection silicon nitride on top of it. The rear side was also passivated by a thermal oxide and was contacted through laser fired contacts (LFC) [49]. The front contacts were realized using photolithographic processes, evaporation of a stack of titanium, palladium and silver (TiPdAg) and subsequent electroplating. The full processing scheme is visualized in Figure 5.25 .

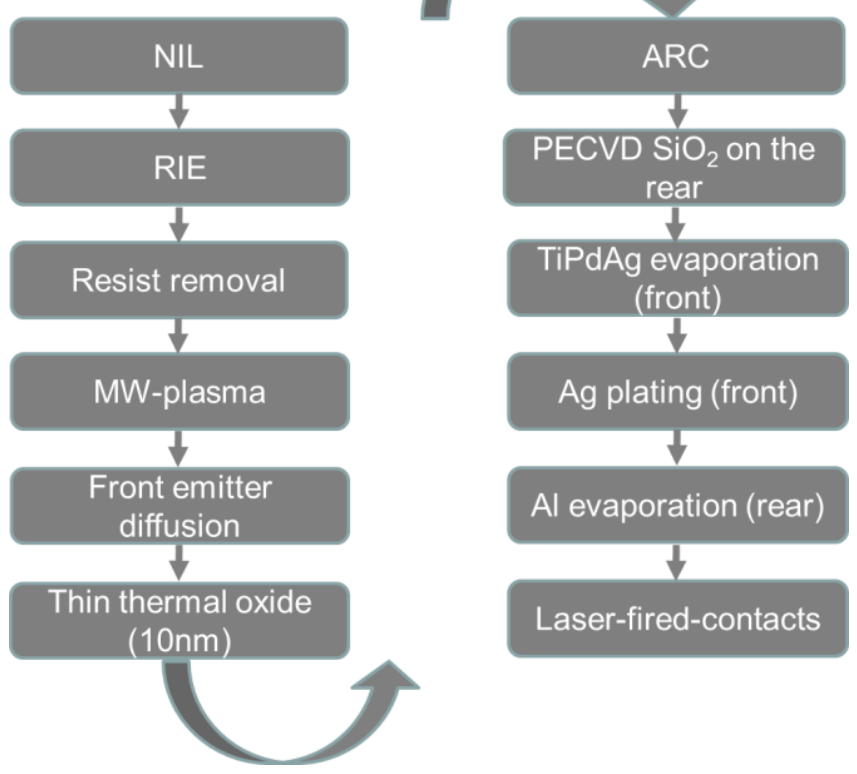

Figure 5.25: Schematic view of the process chain applied for the fabrication of honeycomb textured solar cells on monocrystalline FZ material.

The honeycomb as well as the isotexture was applied on the full 4" wafer area; however, to generate more data, seven cells were processed on each substrate with $2 \times 2 \mathrm{~cm}^{2}$ cell size. Table 5.3 summarizes the results of isotextured references as well as honeycomb textured cells with a single layer ARC (SARC) from [196] and with a double layer ARC (DARC) [143]. It should be mentioned that at the time these cells were fabricated, the reflectivity of the honeycomb-textured cells was not yet as good as shown in the reflection measurement in Figure 5.23 . 
Table 5.3: Solar cell parameters of honeycomb $(8 \Omega \mathrm{cm})$ and isotextured $(0.9 \Omega \mathrm{cm})$ solar cells on $\mathrm{FZ}$ material.

\begin{tabular}{lcccc}
\hline \hline Type of texture & $\begin{array}{c}\mathbf{V}_{\mathbf{o c}} \\
{[\mathbf{m V}]}\end{array}$ & $\begin{array}{c}\mathbf{j}_{\mathbf{s c}} \\
{\left[\mathbf{m A} / \mathbf{c m}^{2}\right]}\end{array}$ & $\begin{array}{c}\mathbf{F F} \\
{[\%]}\end{array}$ & $\begin{array}{c}\mathbf{n} \\
{[\%]}\end{array}$ \\
\hline $\begin{array}{l}\text { Honeycombs (DARC) } \\
\text { Best Cell }\end{array}$ & 628.0 & 40.7 & 72.8 & 18.6 \\
$\begin{array}{l}\text { Honeycombs (SARC) } \\
\text { Best Cell }\end{array}$ & 630.1 & 40.1 & 73.9 & 18.7 \\
$\begin{array}{l}\text { Isotexture (SARC) } \\
\text { Best Cell }\end{array}$ & 657.1 & 38.49 & 77.3 & 19.6 \\
\hline $\begin{array}{l}\text { Honeycombs (DARC) } \\
\text { Average of 4 cells }\end{array}$ & 629.0 & 40.3 & 72.3 & 18.3 \\
$\begin{array}{l}\text { Honeycombs (SARC) } \\
\text { Average of 21 cells }\end{array}$ & 621.7 & 39.6 & 68.2 & 16.8 \\
$\begin{array}{l}\text { Isotexture (SARC) } \\
\text { Average of 14 cells }\end{array}$ & 644.8 & 37.9 & 77.8 & 19.0 \\
\hline \hline
\end{tabular}

The overall very high absorption of the honeycomb textured cells is confirmed by excellent values for the $j_{s c}$ of up to $40.7 \mathrm{~mA} / \mathrm{cm}^{2}$. The overall efficiency of the isotextured substrates is superior to the honeycomb textured ones. This mainly results from the low fill factor, but also from the moderate open-circuit voltage of the honeycomb textured samples. For improving the fill factor, tests have been performed using aerosol printing of the front contacts, which resulted in strongly reduced contact resistances [196]. It was assumed that the very steep sidewalls of the honeycomb texture were not contacted continuously with the evaporated metals. However, it was not possible to see this effect with a microscope after the plating process. Another possible source for the lower fill factors might be related to the higher base resistivity and thus higher contact resistances on the rear side for the LFC process.

To work out the influence of the base resistivity on the cell results, PC1D simulations were performed by Bernhard Michl for the best cells [143]. Figure 5.26 shows reflection measurements, internal and external quantum efficiencies as well as the simulation results for the best isotextured and honeycomb textured cells with SARC. The results for short-circuit current density and open-circuit voltage are in good agreement to the measured values (Honeycombs: $39.0 \mathrm{~mA} / \mathrm{cm}^{2}, 635 \mathrm{mV}$; Isotexture: $37.5 \mathrm{~mA} / \mathrm{cm}^{2}, 660 \mathrm{mV}$ ). Also, the measured and simulated quantum efficiency for an isotextured as well as a honeycomb textured cell are in good 
agreement as shown in Figure 5.26. By changing the base resistivity for the honeycomb textured cell in the simulation to the one of the fabricated isotextured references of $0.9 \Omega \mathrm{cm}$, the $j_{s c}$ decreases by about $0.3 \mathrm{~mA} / \mathrm{cm}^{2}$ to $38.7 \mathrm{~mA} / \mathrm{cm}^{2}$, whereas the $V_{o c}$ increases to a value of $658 \mathrm{mV}$. Thus, the pure optical gain of the honeycomb texture compared to the isotexture for an equal base resistivity of $0.9 \Omega \mathrm{cm}$ is around $1.2 \mathrm{~mA} / \mathrm{cm}^{2}$ and the moderate voltages of the honeycomb textured cells can be attributed to the lower base doping. Additionally, the very high IQE for short wavelengths for the honeycombs indicates that there is no problem with plasma induced damages reducing $S_{\text {front }}$ dramatically. This was also found to be consistent with the PC1D simulations. There, a factor of 2 higher $S_{\text {front }}$ was extracted for the honeycomb texture, which might be attributed to a surface enlargement.

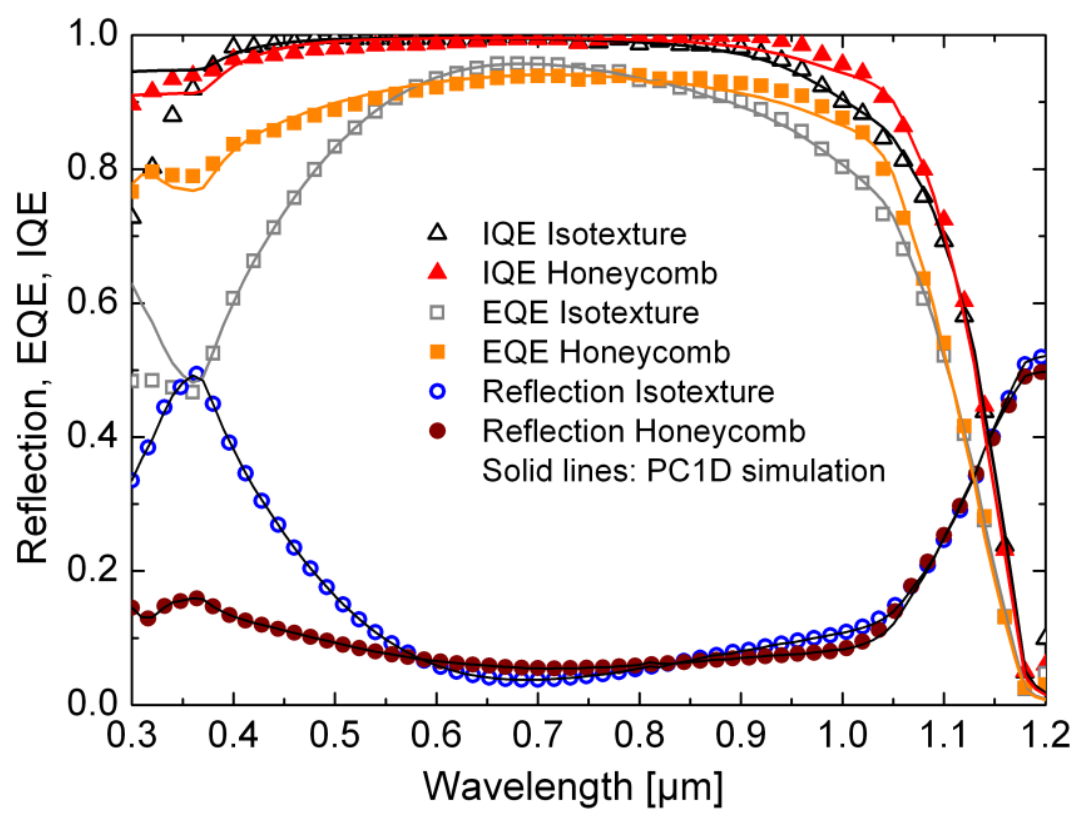

Figure 5.26: Local spectral response measurements (not full cell area) of a honeycomb and an isotextured cell (both SARC) including reflection measurements as well as internal and external quantum efficiencies are shown. The symbols represent measured values; the lines are the result of a PC1D simulation.

\section{Fabrication of large area honeycomb textured mc-Si solar cells using the Roller-NIL tool}

After the proof-of-concept cell fabrication using the conventional NIL tool, an upscaling to the fabrication of $125 \times 125 \mathrm{~mm}^{2}$ large area cells using the Roller-NIL tool was conducted. To avoid the problems concerning the low fill factor that were found for the small area cells, two alternative processes for the front surface metallisation were investigated. The aerosol printing, which was already reported 
before to perform well for contact resistance test samples, and a laser-chemicalprocessing (LCP, [197]) to open the dielectric passivation layer were tested. Both processes are combined with subsequent plating processes. For the material, high quality FZ $p$-type $1 \Omega \mathrm{cm}$ material was again chosen so not to be affected by material quality issues in this development step. The $120 \Omega / \square$ emitter was realised by a $\mathrm{POCl}_{3}$ diffusion. On the rear, an aluminium layer was screen-printed and fired to form an aluminium back surface field (BSF). The choice of an aluminium-BSF cell architecture is motivated by faster processing cycles, because in the first solar cell batches problems in terms of contact resistances or low open-circuit voltages occurred, which were not reproducible. The reference samples were a planar and a random pyramid textured cell. All cells had a single layer ARC. Since for the LCP processed samples problems occurred in terms of contact formation, only results for aerosol printed solar cells are shown in Table 5.4. Furthermore, it is difficult to extract statistical data out of this solar cell batch since for the honeycomb textured cells no uniform process sequence was applied. Besides the testing of contacting schemes, additionally the second etching step (widening of the etching pits) was varied. There, in addition to the microwave plasma etching, also wet-chemical etching as well as a combination of both approaches was tested. The best honeycomb textured cell was obtained for pure microwave plasma etching.

Table 5.4: Results of $125 \times 125 \mathrm{~mm}^{2} \mathrm{FZ}$ p-type $1 \Omega \mathrm{cm}$ cells with Al-BSF.

\begin{tabular}{lcccc}
\hline \hline Type of texture & $\begin{array}{c}\mathbf{V}_{\mathbf{o c}} \\
{[\mathbf{m V}]}\end{array}$ & $\begin{array}{c}\mathbf{J}_{\mathbf{s c}} \\
{\left[\mathbf{m A} / \mathbf{c m}^{\mathbf{2}}\right]}\end{array}$ & $\begin{array}{c}\mathbf{F F} \\
{[\%]}\end{array}$ & $\begin{array}{c}\mathbf{\eta} \\
{[\%]}\end{array}$ \\
\hline Planar (1 cell) & 652 & 33.8 & 61.9 & 13.6 \\
Random Pyramids (1 cell) & 643 & 37.0 & 75.4 & 17.9 \\
$\begin{array}{l}\text { Honeycomb (best cell, } \\
\text { best values for each parameter in brackets) }\end{array}$ & 632 & 34.9 & 76.0 & 16.7 \\
\hline \hline
\end{tabular}

From the moderate values for $j_{s c}$ it can be concluded that the texturing process for this solar cell batch was not entirely successful. However, the low level of these values is also caused by the low internal reflection properties of the aluminium BSF rear. In [89] it is found that the light trapping of honeycomb textures is even higher than for pyramidal textures. Having bad internal reflection properties this effect is diminished. This could be one reason leading to the honeycomb texture not showing it's full strength in this aspect. Still, the texture quality was found to be suboptimal. For the wet-chemical etching, it was found that the texture quality was locally better than for the microwave plasma etching, but the homogeneity was lower. 
The values for $V_{o c}$ showed a large deviation, which was on a reasonable level for an aluminium-BSF rear side. There was even one sample with a very high voltage of $651 \mathrm{mV}$ (this was reached for a combination of microwave plasma and wetchemical etching for the widening of the etching pits; the $j_{s c}$ for this sample was $\left.34.9 \mathrm{~mA} / \mathrm{cm}^{2}\right)$. This value for $V_{o c}$ is higher than for the random pyramids sample and even in the same range as the planar reference. A similar characteristic was found for the fill factor of the cells. The best value of $77.7 \%$ shows that the contacting using aerosol printing and plating was successful.

\subsubsection{Summary and next steps}

It was shown that the process chain of NIL and plasma etching can be applied to realise honeycomb textures in silicon of a very high optical quality (down to $14 \%$ weighted reflectance without ARC on multicrystalline silicon). This was demonstrated using both the conventional as well as the newly developed Roller$\mathrm{NIL}$ tool. For the latter one the patterning of etching masks was demonstrated on substrate sizes up to $156 \times 156 \mathrm{~mm}^{2}$.

Using the conventional tool, $2 \times 2 \mathrm{~cm}^{2}$ PERC solar cells on high-efficiency FZ material were fabricated. There, very high short-circuit current densities of up to $40.7 \mathrm{~mA} / \mathrm{cm}^{2}$ were reached (DARC). IQE measurements and PC1D simulations of the realised cells have shown that the texturing process does not introduce significant damages (plasma damages or contaminations). Thus, the honeycomb texturing based on the presented process chain is suitable for the fabrication of high-efficiency multicrystalline silicon solar cells.

Afterwards, the up-scaling to large area cell processing using the Roller-NIL tool was investigated. There, the adaptions in the process chain had to be implemented in terms of etching processes and resist removal. First cell batches suffered from non-reproducibilities in terms of deviations of minority carrier lifetimes. After finding a stable process, the feasibility of large area processing was demonstrated on an FZ $p$-type aluminium BSF batch using $125 \times 125 \mathrm{~mm}^{2}$ wafers. Unfortunately, the texturisation process was not optimal in this batch and also a high deviation of resulting IV-parameters was obtained. This was related to inhomogeneous optical properties of the texture. Still, isolated best cell parameters verified the feasibility of this process chain on basis of the Roller-NIL tool.

As future steps for the honeycomb texturing, there are already on-going cell batches to verify the high-efficiency potential on multicrystalline silicon based on the PERC structure. One important issue is the up-scaling of etching processes to larger substrates or even multi-wafer processing. This is especially important, as it was found that despite having a better homogeneity in the etching mask patterning using the Roller-NIL tool compared to the conventional one, the quality 
of the final texture was not very stable. One potential source might be related to the plasma tool applied within this work. Besides the etching processes, there is also on-going research into the contact formation on the front side based on laser chemical processes [197].

Another research topic that has been started within this work is the integration of features into the stamp, which allow the realisation of plateaus for a latter metallisation of solar cells. This could even be used to realise a kind of buried contacts without any additional process steps. To this end, a combination of NIL and photolithography processes was used to integrate spacings into the periodically patterned "master structures". From these newly realised master structures, silicone stamps were replicated and imprints were performed using the conventional NIL tool and SU8 resist on glass substrates. Figure 5.27 shows SEM micrographs of these replicated features integrated into an etching mask. It can be seen that additional process optimisation has to be conducted in terms of residual layer thicknesses, but the $30 \mu \mathrm{m}$ wide spacings for contact fingers are replicated very well.
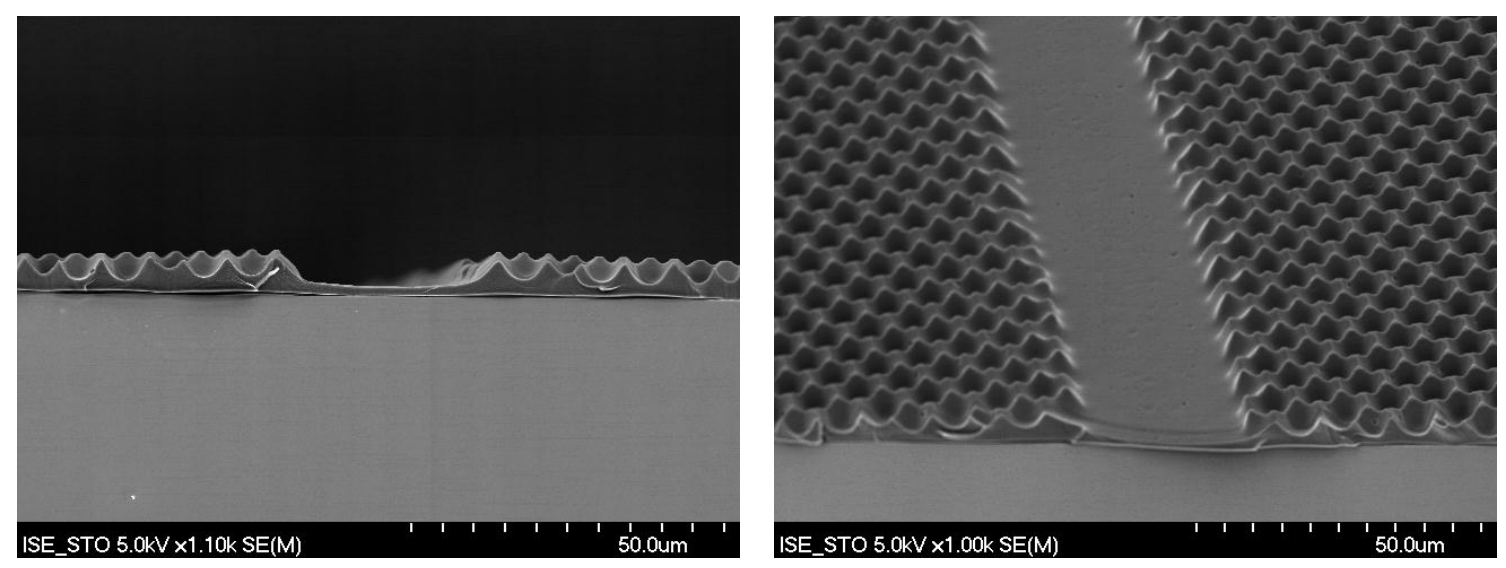

Figure 5.27: SEM micrographs of an imprinted etching mask on a glass substrate with integrated $30 \mu \mathrm{m}$ wide spacings for contact fingers in future solar cells. 


\subsection{Diffractive gratings on the back side of silicon solar cells}

\subsubsection{Description of this concept}

The basic of introducing diffractive elements into solar cells, as well as the relevant physics, was already explained in subsection 3.2.2. The design of the diffractive elements realised within this work is based on the theoretical works of Peters et al. [64] and Mellor et al. [63, 95]. The concept to implement photonic structures on the wafer rear side was originally introduced by Morf et al. [98].

Peters and Mellor published results of wave optical simulations of gratings on the wafer rear side, indicating that the optimum structure period is around $1 \mu \mathrm{m}$ (see Figure 5.28, [64]). In [99] this result also was confirmed by simulations. There thickness-dependent studies were performed, which showed that for silicon thicknesses from 100 to $200 \mu \mathrm{m}$, which are reasonable for wafer based solar cells, the optimized period is around $950 \mathrm{~nm}$. Furthermore, the results of Mellor show that theoretically bi-periodic patterns perform better than uni-periodic patterns. This shall also be investigated experimentally within this work.

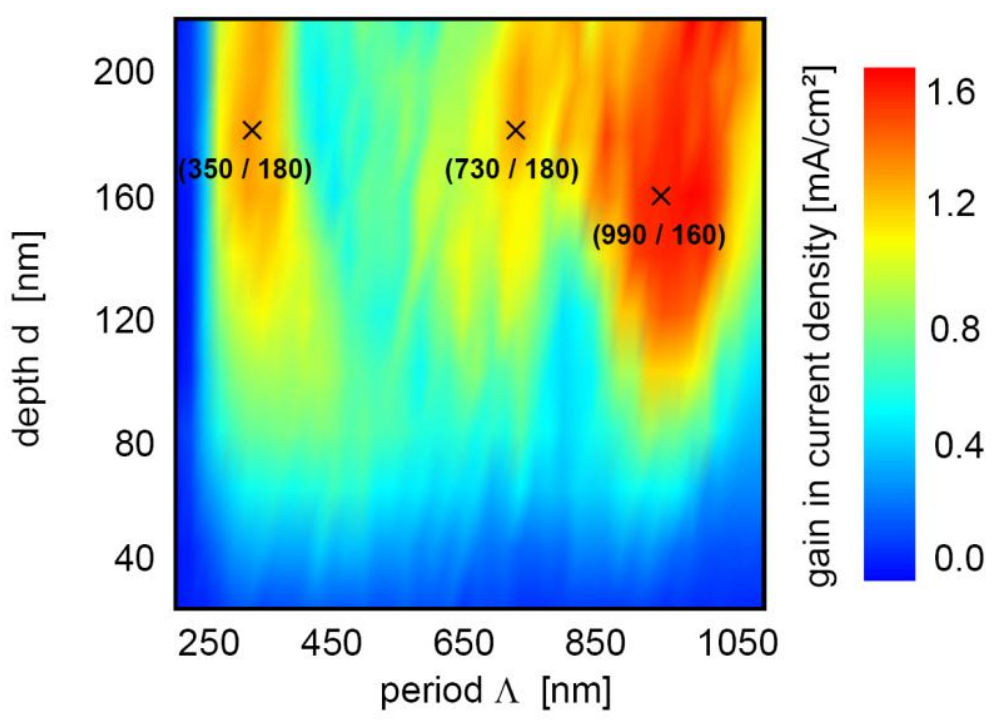

Figure 5.28: Wave optically simulated gain in absorbed photocurrent density of a sample with a grating on the rear compared to a flat reference. The varied parameters are the grating period and the depth. The simulated structure is a $40 \mu \mathrm{m}$ thick silicon solar cell with a flat front, a silicon nitride $A R C$, and an ideal, non-absorbing, dielectric back reflector. Points of local maxima are highlighted and the corresponding parameters are given. Graph taken from [64].

These underlying calculations both of Peters et al. and Mellor et al. were performed for silicon solar cells with flat front surfaces. This is mainly related to the simulation approach applied in these works, which is based on the rigorous coupled wave analysis (RCWA). In principle it can be expected that implementing a photonic structure on the rear of a solar cell with a flat front will not outperform 
the optical performance of classical pyramidal front side textures ${ }^{15}$. And consequently one should aim for a combination of a pyramidal texture with a photonic rear side structure. A patent was filed for the combination of a front $\mathrm{V}$ groove texture with a linear diffraction grating on the rear that is oriented perpendicular to the grooves [198].

However, within this work, samples with a flat front were investigated for several reasons: (i) to allow a better comparison with the theoretical works of Peters et al. and Mellor et al.; (ii) the absorption enhancement is more pronounced for flat front surfaces and thus the study of this effect can be investigated more easily; (iii) polarisation optical effects can be investigated more thoroughly (iv) one important issue is the electrical quality of the nano-patterned rear side and this can also be studied independently from a front side texture; ( $v$ ) no alignment to front side structures is necessary; and finally (vi) after setting up a process chain to fabricate a photonic rear side structure without degrading the electrical quality of the solar cell rear, this concept can easily be transferred to solar cells with a textured front side.

The experimental proof of efficiency enhancements on a high baseline level efficiency introduced by diffractive rear side structures cannot be found in the literature up to now. This is mainly related to a degradation of the electrical quality of nano-textured surfaces realised via plasma etching [199, 200]. With new surface passivation technologies as enabled e.g. by atomic layer deposition (ALD), these problems might be overcome. Just recently, it was shown that this technology even allows for the passivation of black silicon [112]. We also aim for a concept using ALD for the deposition of a passivation layer. However, we aim to combine an optically active layer on the wafer rear, without affecting the best achievable properties for electrical passivation. To this end, we maintain a planar passivated rear side. Thereby, the best possible passivation quality can be realised. Then, on this passivated layer, an amorphous silicon (a-Si) layer is deposited, which is later patterned. After that, a dielectric buffer layer (e.g. made of silicon oxide) and the aluminium rear reflector are deposited. In a solar cell, the silicon bulk might be contacted with the aluminium locally e.g. by laser-fired contacts. This setup allows the decoupling of the electrical and optical properties, since the optically active grating is not fulfilling any electrical purposes. A patent was also filed regarding this decoupling of electrical and optical effects [201].

There are some design rules concerning the layer thickness and topography, which have to be obeyed for this setup. First, the passivation layer should be as thin as

15 This statement only holds for established single layer antireflection coatings made of silicon nitride. 
possible $(<10 \mathrm{~nm})$ to minimise any optical influences i.e. reflection. The a-Si layer should be so thin, so that a local contact firing process can be applied. On the other hand, the grating depth determines the minimal a-Si thickness, in that a thin a-Si layer must still be maintained in the recessed areas after the plasma etching so that the $\mathrm{Al}_{2} \mathrm{O}_{3}$ passivation layer remains protected. Because of the bigger band gap energy of a-Si, the long wavelength radiation, which is all that impinges on the wafer rear side, cannot be absorbed in this layer. The dielectric layer used as an optical buffer layer between the a-Si grating and the aluminium absorber from the optical point of view of damping non-propagating modes should be as thick as possible. The reason is that evanescent modes introduced by the grating (or by oblique incidence exceeding the critical angle for total internal reflection) should be damped as much as possible to minimise parasitic absorption in the metal reflector. However, the optimum thickness to achieve best reflectance properties additionally is affected by Fabry-Pérot effects [202]. A second aspect to minimise this unwanted type of absorption is that the interface between dielectric and metal should be as planar as possible, for it is known that corrugated metal surfaces are lossy reflectors [203]. Another requirement set for the thickness of the dielectric layer is related to the contact firing process. Overly thick layers should be avoided, so that a laser firing of contacts is possible. For the metal reflector, processes should be applied that lead to a smooth surface quality (e.g. evaporation).

The investigations on the realisation of diffraction gratings were performed on bright etched FZ wafers. Therefore, the focus will not have to be placed so much on the adaptability to rough surfaces. Still, NIL processes on large areas require the use of flexible stamp materials to achieve homogeneous residual layer thicknesses. As a target for the grating geometry, binary shapes with a duty cycle of $50 \%$ were aimed for. This corresponds to the geometries investigated in the theoretical works referenced before.

\subsubsection{Interference lithography and stamp fabrication}

\section{Interference lithography}

Within this work, master structures of $1 \mu \mathrm{m}$ period with linear and crossed gratings were fabricated according to the theoretical works listed before. The basics of the process of laser interference lithography (LIL) are explained in section 4.2. This process was applied for the fabrication of polymeric master structures with these periodic structures within the framework of the master thesis of Aron Guttowski [204]. The detailed description of the processing is given there. The processing of these master structures on glass substrates comprised of the steps: 
- cleaning process sequence

- spin coating of a bottom anti reflection layer (BARC)

- baking process on a hot plate

- spin coating of a positive tone photoresist

- soft bake on a hot plate

- the actual lithography process

The periodic features were realised in different pattern heights between 300 and $500 \mathrm{~nm}$ on $75 \times 75 \mathrm{~mm}^{2}$ glass samples. Therefore, corresponding film thicknesses of the positive tone resist were realised in this range. For these small film thicknesses, a BARC layer is essential, especially when exposing with a highly coherent light source. This helps to minimise the formation of standing wave effects within the resist due to the superposition of incoming and reflected laser beams. After the spin coating of the BARC layer, solvents are driven out by a baking process. On this BARC layer the resist is applied. We used the positive tone resist AZ-MIR701 ${ }^{\mathrm{TM}}$ for the fabrication of the surface reliefs. After the spin coating and a softbake, in which an appropriate solvent content within this resist shall be adjusted, the actual LIL process follows. To realise the wanted types of periodicity, a two beam LIL process was applied. While a single exposure leads to a linear uniperiodic pattern, a twofold exposure with a rotation of the sample of $90^{\circ}$ between the exposures using the same setup leads to a crossed grating. After the exposure process, the development follows, in which photo-chemically modified volumes of this resist can be removed. This takes place in an aqueous alkaline solution. The resulting patterns of the master structures are exemplarily shown in Figure 5.29.
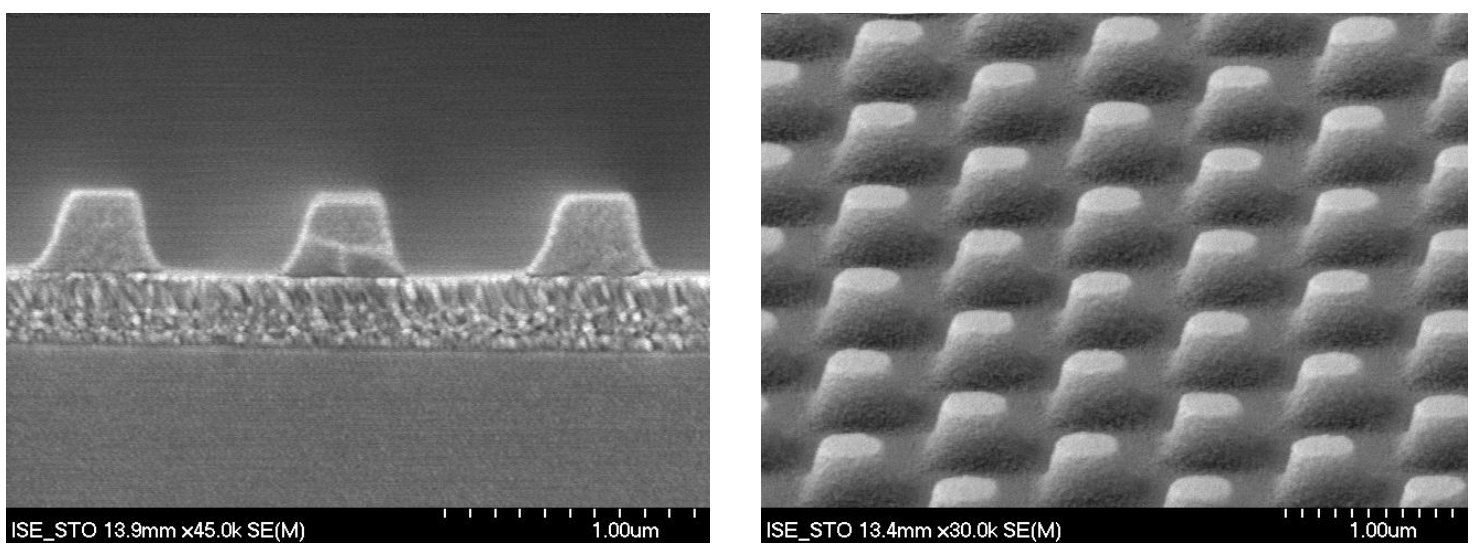

Figure 5.29: SEM micrographs of photoresist structures realised via interference lithography. On the left hand side a linear grating is shown in cross section and on the right hand side a tilted view of a crossed grating is shown. In both cases the period is about $1 \mu \mathrm{m}$ and the pattern depth is about $350 \mathrm{~nm}$. The sample size was $75 \times 75 \mathrm{~mm}^{2}$. 


\section{Stamp fabrication}

As already described in section 4.3 and 5.1.2, the stamps to be used in the following NIL process shall be soft (to enable a conformal adaption to wavy surfaces) and UV-transparent (optical curing of the crosslinking resist used in NIL). Again, mainly PDMS materials were evaluated for this purpose. First, commercially available products of Wacker and Dow Corning were tested. The Young's modulus of these silicone materials is in the range of $2 \mathrm{MPa}$. These materials are very simple in terms of handling and processing and were also used for the fabrication of etching masks for the honeycomb patterning as described before. However, for decreasing pattern dimensions, problems occur in terms of feature stability. This effect already mentioned in section 4.3 leads to a so-called pattern collapse for aspect ratios exceeding a particular value for a given structure period, shape and duty cycle as well as the Young's modulus of the material. A simplified model to calculate critical aspect ratios for binary patterns is described in the dissertations $[145,205]$. We found that for the pattern dimensions applied within this work ( $1 \mu \mathrm{m}$ period), we were just at the borderline using the standard types of PDMS. While samples of a pattern height of around $300 \mathrm{~nm}$ were replicated very well, a pattern collapse was found to occur for samples in the range of $500 \mathrm{~nm}$. Therefore, research was conducted to find materials that allow a shift of this physical borderline by increasing the Young's modulus of the stamp material. Characteristics like UV-transparency have to be maintained. The adaptability to non-planar surfaces suffers when increasing the Young's modulus. Thus, a compromise has to be found.

Materials tested to improve the stamp fabrication for the UV-NIL process, which can also be found in the literature were COC [206], ETFE [147, 166, 207] and modified PDMS [122, 146].

The commercially available COC used for the investigations was the Topas 5013 from Topas Advanced Polymers. This thermoplastic material can be bought in thin sheets of about $1 \mathrm{~mm}$ thickness. The patterning of this material is realised using hot embossing processes. Positive characteristics of this material are its very high UV-transparency, its high pattern fidelity of replicated features and its chemical resistance against solvents as well as acidic and alkaline solutions. Also, its Young's modulus of 3.2 GPa is by far higher than for elastomeric PDMS materials [208]. Disadvantages found within the present study were related to sticking behaviour of resists to this stamp material during the NIL process, the adaptability to rough or wavy surfaces was not convincing and this material is virtually not permeable to gases. This last point is critical when applying solvent assisted imprint processes as described in subsection 4.3.3, but can also be crucial in terms of trapped air beneath the stamp during imprinting. 
There are some publications showing impressive imprint results using ETFE as a stamp material $[147,166,209]$. One big advantage for this stamp material is the intrinsic anti-sticking behaviour of this Teflon-like fluoropolymer. This material is commercially available as a foil material e.g. by DuPont or Asahi Glass Company and values for the Young's modulus can be found in the literature that are in the range of $190 \mathrm{MPa}$ to $1 \mathrm{GPa}[147,209]$. The patterning again takes place in a hot embossing process. Conditions for this replication process can be found in [166, 207]. In Figure 5.30, AFM measurements of replications in different materials of a linear grating with a period of $410 \mathrm{~nm}$ and a pattern depth of about $260 \mathrm{~nm}$ are shown. In Figure 5.30 a) the results of the soft PDMS Elastosil RT601 are shown. It can be seen, that a pattern collapse occurs. As can be seen in Figure $5.30 \mathrm{~b}$ ) these small features with high aspect ratios can be replicated into ETFE. There, as a result of the high Young's modulus, no problems related to a pattern collapse were seen. Within this work, the anti-sticking behaviour of this material was found to work very well for the demoulding within the imprint process; however, during the patterning of the ETFE via hot embossing using nickel masters, no good and reproducible results were obtained. Mainly the homogeneity was found to be very critical when replicating large area masters. Often features seemed to be torn off during the demoulding and kept sticking in the master structure (see Figure $5.30 \mathrm{c})$ ). This might be related to crystallisation effects as a result of not optimised temperature and pressure setting during the processing [207]. However, changes in these parameters did not yield significant improvements. Other disadvantages of this material are that it is difficult to fabricate homogeneous stamps in terms of applying the pressure on this foil in a uniform process. We tried to fabricate a stack consisting of a soft PDMS bulk onto which the ETFE foil is mounted. However, no process could be found to effectively bond these materials together. As with COC, ETFE also has virtually no gas permeability. 

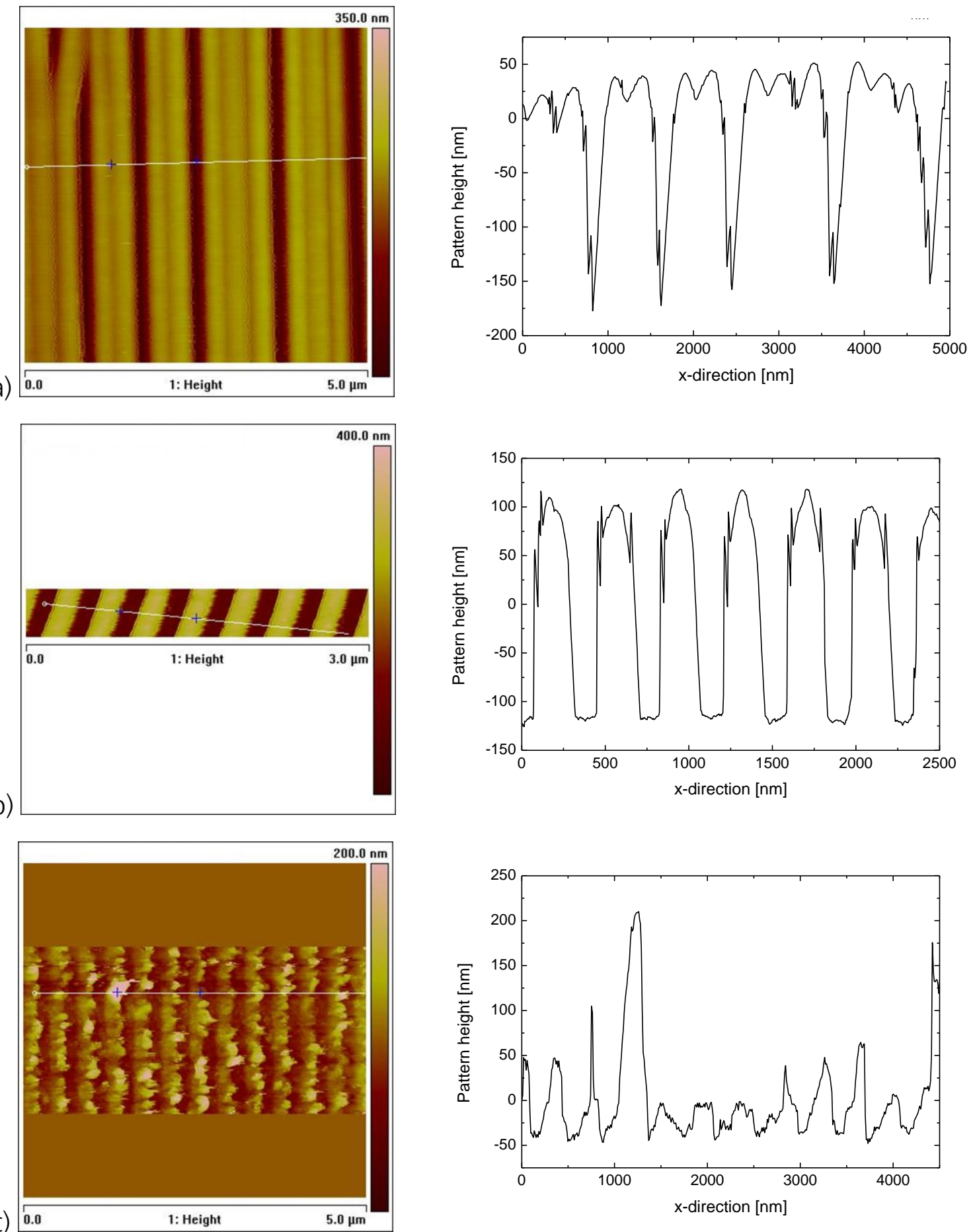

Figure 5.30: Contour plots and cross-sections of AFM measurements of replicated stamps using different materials. The master structure is a linear grating with a period and depth of about $410 \mathrm{~nm}$ and $260 \mathrm{~nm}$, respectively. a) Resulting pattern collapse using a soft PDMS material (Elastosil RT601). In b) and C) ETFE was used as material for the replication process. b) shows the very high quality that can be achieved using this material; however, in c) a very rough surface and a poor replication quality was found for very similar processing conditions. 
As mentioned before, the anti-sticking behaviour as well as the high Young's modulus of ETFE in principle allows the replication of very small features via NIL. This was evaluated using self-organised pores in anodic alumina as a master structure [118]. From this master structure, an ETFE stamp was replicated using hot embossing. The ETFE foil was placed beneath a block of soft PDMS during the NIL process using mr-UVCur06 as resist. Figure 5.31 shows an SEM micrograph of the patterned resist layer on top of a glass substrate. It can be seen that the very small features (diameters in the range of $100 \mathrm{~nm}$ ) are replicated very well with depths of around $300 \mathrm{~nm}$. Still, the negative aspects of reproducibility and inhomogeneity outweighed these impressive results.

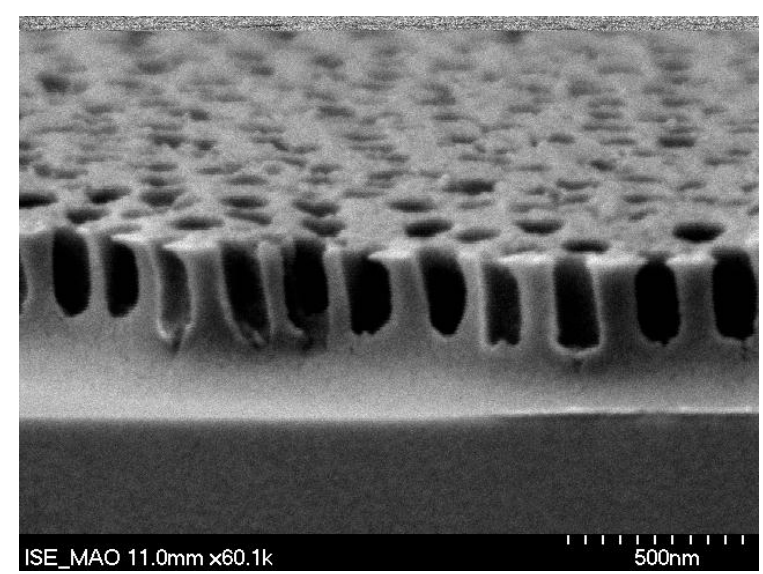

Figure 5.31: SEM micrograph of a NIL patterned resist layer. The master for this structure was fabricated using self-organisation processes in anodisation of aluminium. Hot embossed ETFE was used as the stamp material and mr-UV-Cur06 as the resist material. The pores have diameters and depths of around $100 \mathrm{~nm}$ and $300 \mathrm{~nm}$ respectively.

In [122, 146], so-called h-PDMS compositions are described, which allow the fabrication of PDMS types with higher values for the modulus up to around $10 \mathrm{MPa}$. In [210], we found, that this material is very brittle compared to commercially available types of PDMS. Therefore, it becomes necessary to fabricate stacks of PDMS materials to obtain the best possible behaviour. In [148], the fabrication of a stack comprising of a thick bulk of soft standard PDMS is described, which is bonded to a quartz carrier substrate, onto which a thin layer of this h-PDMS is bonded. This thin h-PDMS layer carries the surface pattern. This setup combines the advantages of the soft PDMS of adaptability to uneven surfaces with the pattern fidelity of the harder thermoplastic materials described before. Even more, this setup allows the maintenance of gas permeability (even if reduced compared to the standard PDMS) and the intrinsic good anti-sticking behaviour of PDMS in general. Of course, this h-PDMS is still an elastomeric material and has a limited modulus. Therefore, the critical feature size for a given aspect ratio is reduced, but still might not be satisfactory when it comes to 
imprinting sub-100 nm features. In the dissertation of M. Verschuuren, which was published just recently, the development of so-called X-PDMS is described [145]. This material is supposed to be fabricated having values for the modulus of up to $80 \mathrm{MPa}$. In general, all the PDMS formulations used within this work, the commercially available Elastosil RT601, Sylgaard 184 and the h-PDMS, are addition curing systems based on two types of pre-polymers, a platinum catalyst and a modulator, which is responsible for manipulating the reaction kinetics.

The fabrication of these multilayer stamps follows the description in [148]. The single steps of this process are:

- cleaning sequence of carrier quartz glass

- spin coating and drying of a primer to achieve an adhesion between PDMS and quartz

- casting and slight thermal curing of a standard soft PDMS bulk onto the quartz substrate

- spin coating of the thin h-PDMS layer onto the master

- assembling the sandwich of quartz/PDMS bulk/h-PDMS/master

- thermal curing of the silicone materials

- demoulding of the master

The feature sizes, which had to be realised within the present work, are in the range of what is possible using the h-PDMS formulation. Because of the advantages of this material compared to the alternatives described before, the focus for the further processing was put onto this material. Figure 5.32 shows the result of an AFM measurement of a replication in h-PDMS. It can be seen that for the h-PDMS the pattern collapse can be avoided even for very small features of $300 \mathrm{~nm}$ period and a pattern depth of about $300 \mathrm{~nm}$.
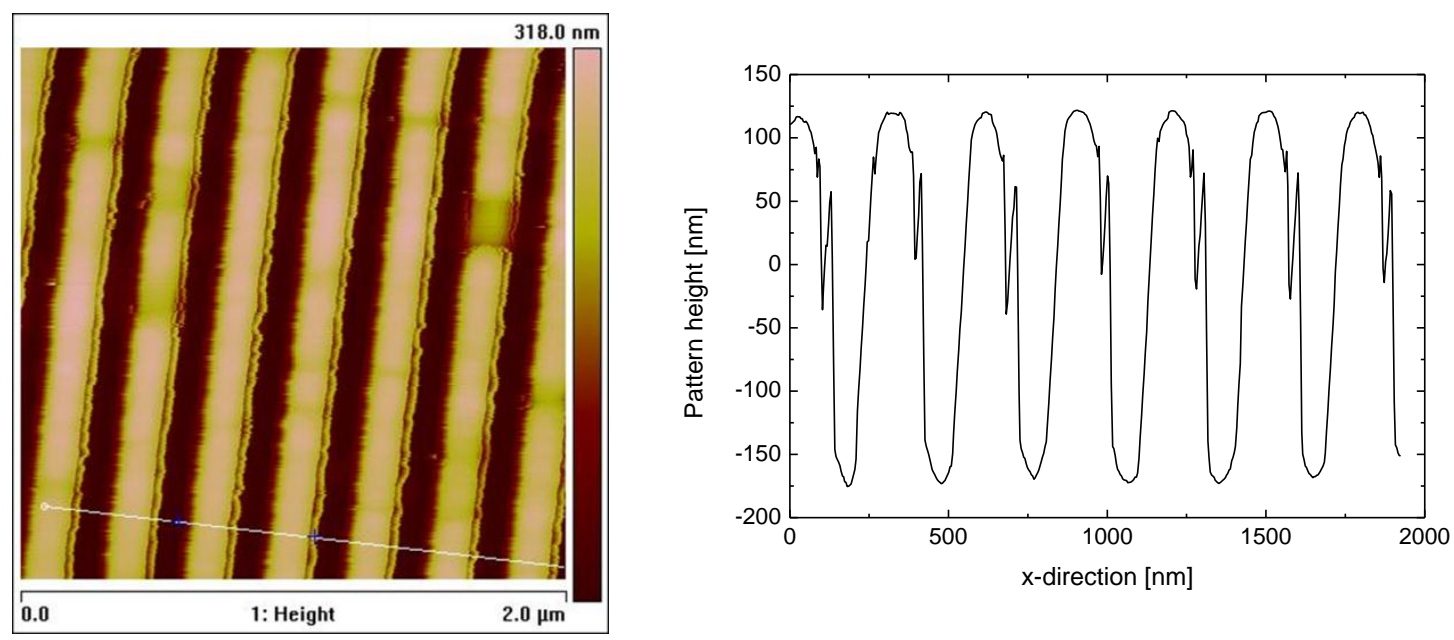

Figure 5.32: AFM measurements of a replicated stamp in h-PDMS. The spikes in the right flanks both in the contour plot (left) as well as in the section (right) are measurement artefacts. 


\subsubsection{Nanoimprint lithography (NIL)}

The NIL process again was set up using the conventional NIL tool based on a planar stamp setup. The up-scaling of the imprint process for the fabrication of rear side diffraction gratings to the Roller-NIL tool was not fully realized within this work. However, first successful results of this up-scaling step are shown at the end of this chapter.

\section{Conventional NIL}

Having fabricated the stacked PDMS stamp as described before, the development of the actual NIL process is the next step. Therefore, appropriate resist materials have to be identified. Critical characteristics are adhesion to the stamp and to the substrate, UV-curability and low viscosity (especially for thin layers / small features). A process was set using the material mr-UVCur06 from Micro Resist Technology [152]. This resist is a low viscous free-radical curing material especially designed for UV-NIL processes. The adhesion promoter Omnicoat as well as the resist were applied via spin coating according to the datasheets [211, 212]. The processing sequence is summarised in Table 5.5 .

Table 5.5: Conditions for the processing of the adhesion promoter, the resist as well as the NIL process.

\begin{tabular}{l|l|l}
\hline \hline Process & Process sequence / Purpose & Conditions \\
\hline \multirow{3}{*}{$\begin{array}{l}\text { Spin coating / } \\
\text { adhesion promoter }\end{array}$} & Remove dust / particles & $4000 \mathrm{rpm}, 10 \mathrm{~s}$ \\
\cline { 2 - 3 } & $\begin{array}{l}\text { Apply adhesion promoter } \\
\text { Spread }\end{array}$ & $50 \mathrm{rpm}, 10 \mathrm{~s}$, \\
\cline { 2 - 3 } & Spinning I & $500 \mathrm{rpm}, 5 \mathrm{~s}$ \\
\cline { 2 - 3 } & Spinning II & $3000 \mathrm{rpm}, 30 \mathrm{~s}$ \\
\hline \multirow{2}{*}{$\begin{array}{l}\text { Baking of adhesion } \\
\text { promoter }\end{array}$} & Hotplate / driving out solvents & $180^{\circ} \mathrm{C}, 2 \mathrm{~min}$ \\
\hline \multirow{2}{*}{$\begin{array}{l}\text { Spin coating / } \\
\text { resist }\end{array}$} & Remove dust/ particles & $4000 \mathrm{rpm}, 10 \mathrm{~s}$ \\
\cline { 2 - 3 } & Apply resist / Spread & $50 \mathrm{rpm}, 10 \mathrm{~s}$, \\
\cline { 2 - 3 } & Spinning & $1500 \mathrm{rpm}, 2 \mathrm{~min}$ \\
\hline Softbake & Hotplate & $100^{\circ} \mathrm{C}, 1 \mathrm{~min}$ \\
\hline \multirow{2}{*}{ NIL } & Pressure / Holding time & $0.4 \mathrm{bar} / 5 \mathrm{~min}$ \\
\cline { 2 - 3 } & Exposure /Curing of the resist & $16: 00 \mathrm{~min}\left(\sim 1500 \mathrm{~mJ} / \mathrm{cm}^{2} @ 365 \mathrm{~nm}\right)$ \\
\hline \hline
\end{tabular}

Very low residual layer thicknesses were achieved both for the crossed as well as the linear gratings. Figure 5.33 shows a photograph as well as SEM images of these samples. The patterned area was around $70 \times 70 \mathrm{~mm}^{2}$. The developed 
processing scheme based on the conventional NIL tool was applied to later process silicon samples as described in the following sections.

a)

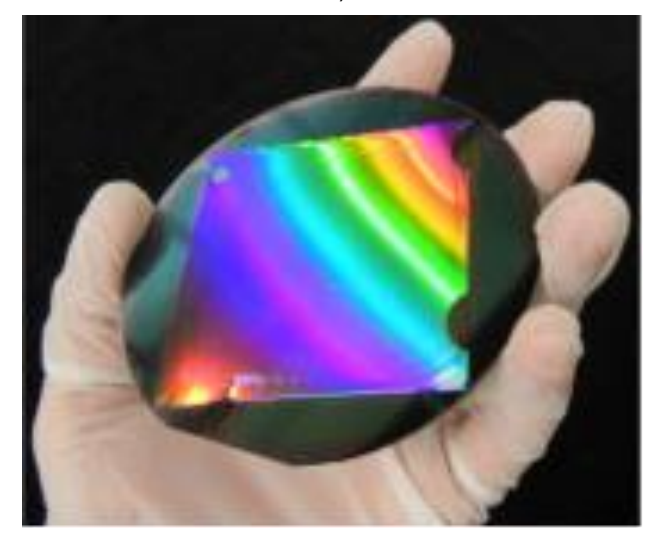

c)

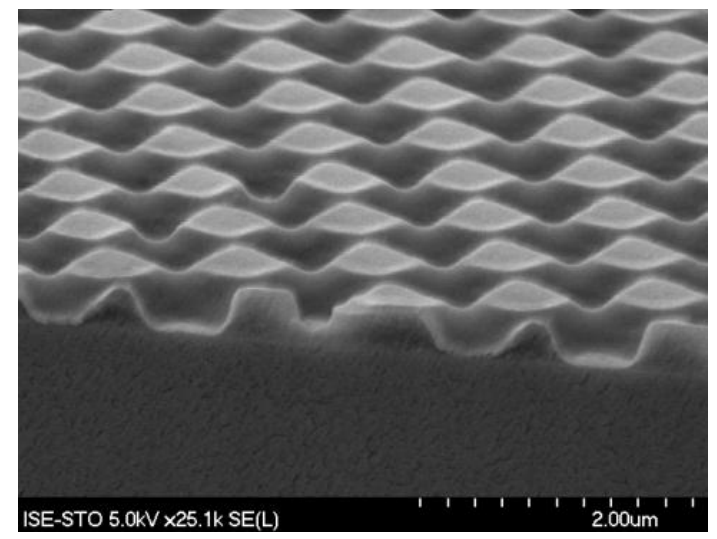

b)

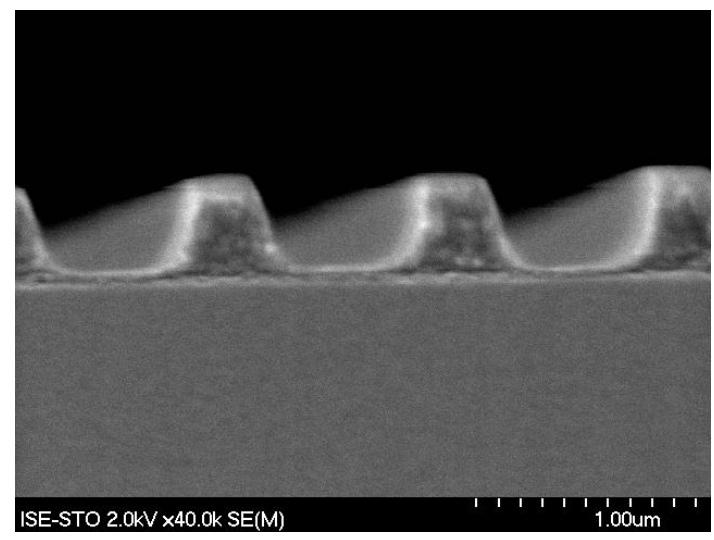

d)

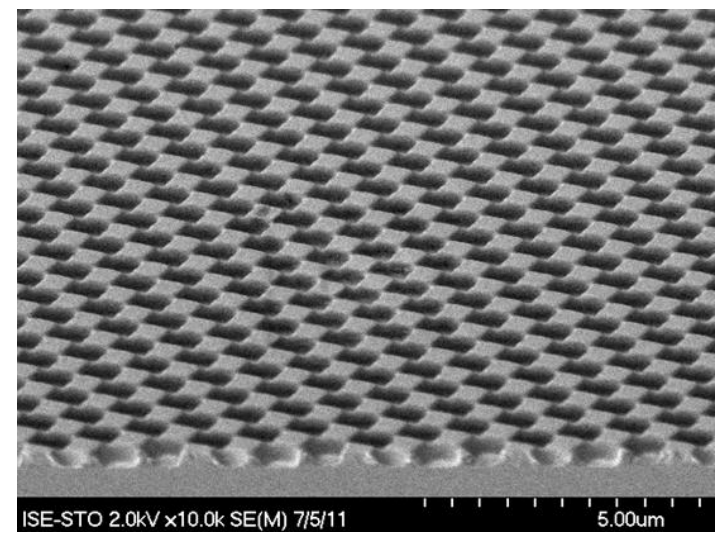

Figure 5.33: a) Photograph of an NIL patterned etching mask on a monocrystalline silicon wafer. The realised structure is a $1 \mu \mathrm{m}$ period linear grating as can be seen in the SEM micrograph shown in b). The micrographs in c) and d) show different magnifications of $1 \mu \mathrm{m}$ period crossed gratings. In all cases the patterned area is around $70 \times 70 \mathrm{~mm}^{2}$ and very low residual layer thicknesses below $50 \mathrm{~nm}$ were achieved.

\section{Roller-NIL}

The up-scaling of imprinting processes for the application of the resist mask patterning for the fabrication of diffractive rear side gratings was investigated on a proof-of-principle basis within this work. Here, basic tests concerning the transferability of the gained knowledge for stamp fabrication and NIL learned from the conventional NIL tool to the newly developed Roller-NIL tool were performed. To this end, master structures with linear as well as crossed gratings with relatively shallow features (below $350 \mathrm{~nm}$ ) were applied for the stamp fabrication. This allows the use of single layer PDMS stamps made of the commercially available standard PDMS without having problems concerning a pattern collapse. Both types of gratings (linear and crossed) were replicated together onto large stamps. 
Therefore, the imprint process of both types of gratings could be investigated for identical NIL and sample conditions.

As imprint resist we chose the solvent free, free-radical curing system Laromer PO84F from BASF. This resist in principle has too high a viscosity to achieve small residual layer thicknesses, but has proven to be very uncritical in terms of processing. Therefore, no fine tuning of spin coating and imprint conditions were necessary to get first insights of the imprinting behaviour using small features on the novel Roller-NIL tool. Figure 5.34 shows SEM micrographs of the resulting pattern quality of the replicated gratings.

a)

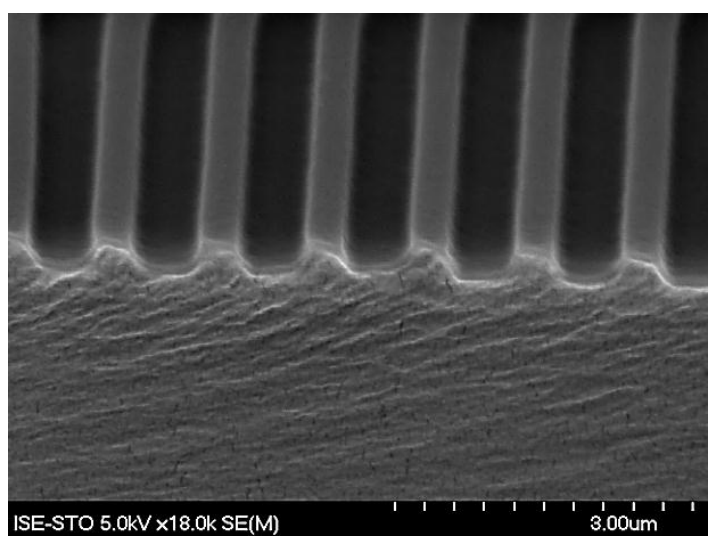

b)

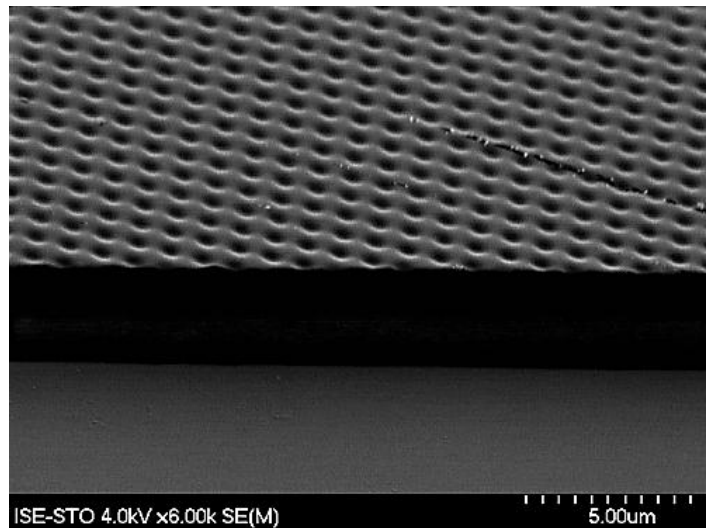

Figure 5.34: SEM micrographs of a) a linear grating and b) a crossed grating of $1 \mu \mathrm{m}$ period replicated via Roller-NIL into Laromer PO84F.

As can be seen in Figure 5.34, the pattern transfer via Roller-NIL was successful. However, it also can be seen, especially for the case of the crossed grating, that the replicated pattern seemed to be rounded and not an exact replication of the master pattern. Furthermore, for both the linear and the crossed grating, the residual layer thickness was determined to be around $4 \mu \mathrm{m}$. Therefore, these patterned resist layers are not suitable as etching masks. Both of these aspects of pattern fidelity as well as residual layer thickness can be optimised. This shall be the scope of future work and was not tackled within this thesis.

\subsubsection{Plasma etching and resist removal}

The pattern transfer of the NIL defined resist mask into crystalline or amorphous silicon was realized using reactive ion etching (RIE). For the removal of resist residues both dry chemical as well as wet-chemical dissolution of a sacrificial layer were tested. 


\section{Reactive lon Etching (RIE)}

The RIE processes investigated for the silicon patterning of diffractive rear side gratings are based on sulphurhexafluorine $\left(\mathrm{SF}_{6}\right)$ as the source for the reactive species. Reactions of fluorine based plasma chemistry with silicon are explained in section 4.4. While for the honeycomb texturing of multicrystalline silicon high etching rates are required to generate around 8 to $10 \mu \mathrm{m}$ deep etching pits, the etching processes for the grating fabrication make different demands. Very precise control of etching rates and anisotropy are necessary. This can be achieved by reducing the pressure in the process chamber. For the same capacitively coupled power, the resulting bias voltage increases drastically. This is due to an increased mean free path length of particles in the plasma. Thereby, very anisotropic etching profiles are possible, which allows the fabrication of nearly binary profile shapes. Another effect of reducing the pressure and thus the density of atoms and molecules in the plasma chamber is that although bias voltages are strongly increased, the etching rate can be kept very low. This is necessary when it comes to the reproducible patterning of gratings of a defined depth. A reason for reducing the pressure and thus creating a stronger physical etching contribution due to the ion bombardment is that the resist we patterned via NIL (mr-UVCur06) was found to have not a very high chemical etching resistivity in oxygen plasmas. Having very low residual layer thicknesses and a physical sputter etching component, a separate residual layer etching could be left out. This residual layer was immediately removed in the silicon etching process based on fluorine chemistry with an addition of oxygen. Table 5.6 summarises the processing conditions. For better understanding of machine relevant parameters like electrode level it is referred to the description of the used plasma tool in subsection 4.4.1 on page 96.

Table 5.6: Reactive Ion Etching process conditions.

\begin{tabular}{ll}
\hline \hline Process parameters & Machine settings \\
Gas flows & $\mathrm{SF}_{6}: 40 \mathrm{sccm}, \mathrm{O}_{2}: 20 \mathrm{sccm}$ \\
Pressure & $1 \mathrm{~Pa}$ \\
RF power & $300 \mathrm{~W}$ \\
Electrode level & $90 \mathrm{~mm}$ \\
Electrode temperature & $-15^{\circ} \mathrm{C}$ \\
\hline \hline
\end{tabular}

The etching times both for linear and crossed gratings were varied between 36 and 72 seconds. This led to a variation of etching depths between 100 and $260 \mathrm{~nm}$ for the linear and 185 and $320 \mathrm{~nm}$ for the crossed gratings. Therefore, different etching rates were obtained for these different types of patterns. These 
are $230 \mathrm{~nm}$ per minute and $300 \mathrm{~nm}$ per minute for the linear and the crossed grating respectively. This difference in etching rates for identical machine parameters and sample preparation might be explained by two reasons. First, the residual layer thickness is very likely to be different due to different squeeze flow characteristics of the resist as a result of varied stamp topography. Second, the etching rate might be changed because of a different ratio of exposed and masked silicon area. The optical performance of these depth variations was later evaluated by reflection measurements.

\section{Resist removal}

Similar to the removal processes described in the context of the honeycomb texturing of mc-Si, again here a dry chemical approach was compared to a wetchemical approach. However, in the application for the fabrication of diffractive back side gratings, it was found that both removal techniques work and do not show the problems encountered with the honeycomb texturing. This is mainly related to thinner resist layers $(\sim 250 \mathrm{~nm}$ compared to $\sim 3.3 \mu \mathrm{m})$, other resist materials and a higher surface quality of the silicon material applied in this study (bright etched c-Si compared to rough mc-Si).

The wet-chemical approach to remove resist residues for this concept is based on dissolving a sacrificial layer (Omnicoat ${ }^{\mathrm{TM}}$ ). Thus, the adhesion promoter spin-coated before served as the sacrificial layer. Thereby, no additional pre-process is necessary. The thickness of this layer applied as described in Table 5.5 was determined via ellipsometry to be $13 \mathrm{~nm}$. This concept emerged to be problematic within the application of the honeycomb texturing because of the roughness of mc-Si wafers. However, on bright etched c-Si substrates, this technique for resist removal was very reliable.

The plasma ashing of the resist layer was also found to be working efficiently. The very high removal rates described before of up to $2 \mu \mathrm{m}$ per minute were not achievable in this application. There $\mathrm{CF}_{4}$ was used additionally to the oxygen as a process gas to enhance removal rates. However, for the resist removal in this application, we cannot tolerate any isotropic silicon etching, since the grating profiles would get rounded or even levelled out. Therefore, processes solely based on oxygen as the process gas were evaluated (gas flow $80 \mathrm{sccm}$ ). To achieve a high oxygen radical density, a low pressure of $1 \mathrm{~Pa}$ and a high power of $1000 \mathrm{~W}$ was applied for the coupling of energy via the microwave slot antenna configuration (to make use of electron cyclotron resonance conditions, see also section 4.4). To enhance the chemical ashing of resist, the electrode level on which the sample is placed was set very high $(120 \mathrm{~mm})$, so that the sample was very close to the plasma. When acceleration voltages are avoided, additional damages 
due to an ion bombardment do not occur. Figure 5.35 shows textured silicon samples after RIE and resist removal via plasma ashing.
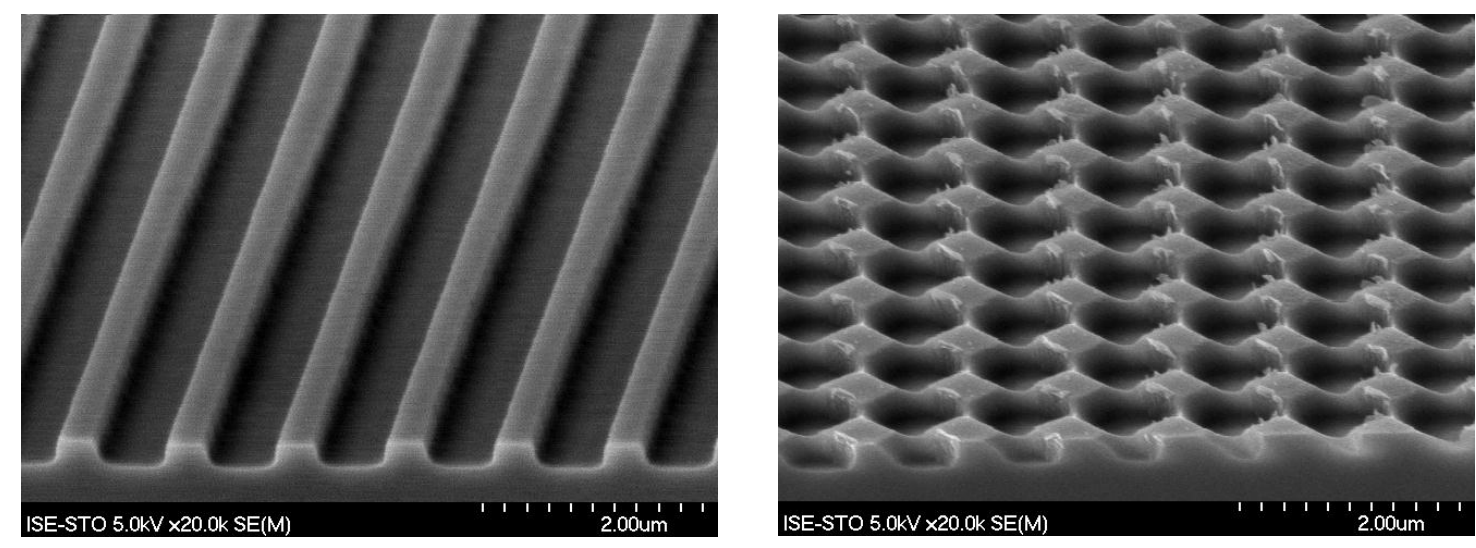

Figure 5.35: SEM micrographs of a linear and a crossed grating after etching and resist removal of depths of about $230 \mathrm{~nm}$ and $300 \mathrm{~nm}$, respectively.

\subsubsection{Optical and electrical characterisation}

For the evaluation of the optical and electrical properties of the textured samples, different types of solar cell pre-cursors were fabricated. In the following, these two structures are explained.

For process development and optical characterisation, the gratings can be directly etched into silicon wafers. Solar cell precursors for determination of absorption enhancements introduced by gratings require a rear reflector, which typically is made of aluminium. To minimise parasitic absorption and optimise internal reflection properties within the semiconductor, commonly dielectric buffer layers are applied between the grating and metal reflector. This test structure, combined with a flat front surface, on which silicon nitride is deposited as antireflection coating (ARC), is applied to study optical absorption enhancements (see Figure 5.36 a).

As explained in section 5.2.1, we aim for a decoupling of electrical and optical properties at the wafer rear side. Therefore, the flat rear side is passivated using a thin $\mathrm{Al}_{2} \mathrm{O}_{3}$ layer, onto which the solely optically active patterned amorphous silicon layer is applied. This concept for integrating a diffraction grating into a silicon solar cell potentially without affecting any electrical properties at its rear side is schematically shown in Figure 5.36 b). In this schematic view, no contacting of the solar cell base is drawn. 
a)

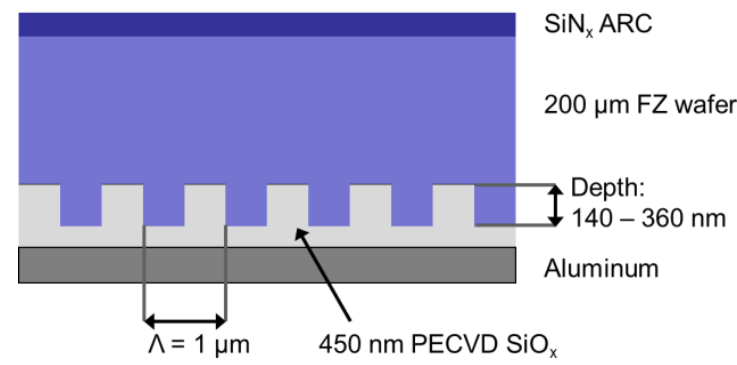

b)

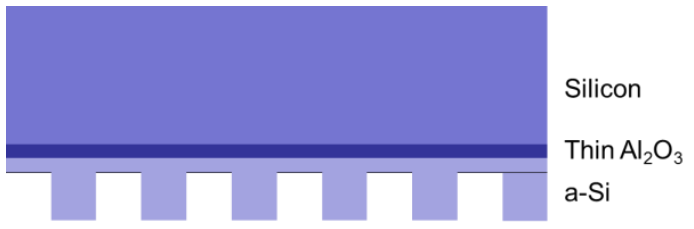

Figure 5.36: a) Sketch of a solar cell precursor structure for optical characterisation. b) Schematic view of the concept to decouple electrically and optically active rear side interfaces.

\section{Optical characterisation}

Linear and crossed gratings were etched into $200 \mu \mathrm{m}$ thick FZ $p$-type $1 \Omega \mathrm{cm}$ silicon wafers using the processes as described before. In a first step, a $450 \mathrm{~nm}$ thick silicon oxide layer was deposited onto the grating via PECVD. The metal reflector on the rear side was realised using thermal evaporation of aluminium. A reference sample was processed having all features but the rear side grating. The reference as well as samples of varying grating depths were then characterised by reflection measurements using a Fourier spectroscopy setup. Since no light is transmitted through the $2 \mu \mathrm{m}$ thick aluminium mirror, the absorption can be calculated via

$$
\text { Absorption }=1-\text { Reflection } .
$$

The resulting absorption measurements of the texturized samples can then be compared to that of the planar reference. Figure 5.37 shows measured spectral absolute absorption enhancements of samples with photonic rear side structure compared to the planar reference. Also shown in the legend of this graph are values for the gain in photocurrent $\Delta j_{p h}$. This value corresponds to all additional photons absorbed due to the introduction of the grating per $\mathrm{cm}^{2}$ and per second. This value can be seen as an upper bound for an achievable gain in short circuit current density, however $\Delta j_{p h}$ includes all parasitic absorption and does not take any relevant solar cell recombination effects into account. The gain in photocurrent density $\Delta j_{p h}$ is calculated via

$$
\Delta j_{p h}=q \int_{980 \mathrm{~nm}}^{1180 \mathrm{~nm}}\left(A b s_{\text {grating }}(\lambda)-A b s_{\text {planar }}(\lambda)\right) \Phi_{A M 1.5 g}(\lambda) d \lambda,
$$

with q being the elementary charge, $A b s_{\text {grating }}$ and $A b s_{\text {planar }}$ being the measured values of the spectral absorption of the grating samples and the reference 
respectively and $\Phi_{A M 1.5 \mathrm{~g}}$ being the photon flux per $\mathrm{cm}^{2}$ and per second of the AM1.5g spectrum.

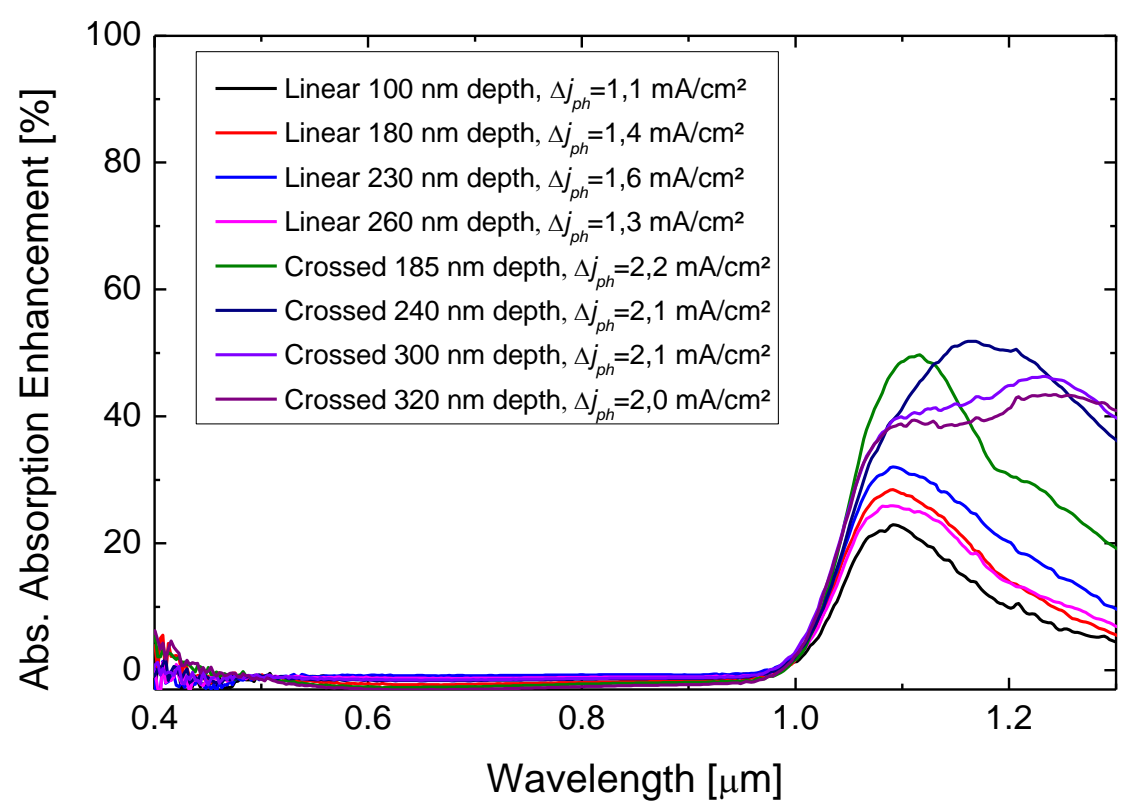

Figure 5.37: Absolute absorption enhancement induced by rear side gratings compared to a planar reference. The values for the gain in photocurrent density are obtained under the assumption that all additional absorption takes place in the silicon.

It can be seen that even for $200 \mu \mathrm{m}$ thick silicon wafers considerable absorption enhancements of up to $52 \%$ absolute can be achieved. Furthermore, maximum values for $\Delta j_{p h}$ of $1.6 \mathrm{~mA} / \mathrm{cm}^{2}$ and $2.2 \mathrm{~mA} / \mathrm{cm}^{2}$ for linear and crossed gratings can be extracted, respectively. Other results that can be concluded from these measurements are that crossed gratings induce a higher absorption enhancement than linear gratings. This conclusion is in agreement with the theoretical considerations published in [95].

The absorption measurements can be translated into path length enhancements introduced by the grating. The necessary penetration depth to achieve the corresponding absorption was calculated for the planar reference as well as the best linear and the best crossed grating sample. To this end, for all samples for the measured absorption, a linear extrapolation from $900 \mathrm{~nm}$ onwards was conducted to remove any influences of light escaping as a result of rear surface reflection. In Figure $5.38 \mathrm{a}$ ) both the measured and the extrapolated curves are shown for the linear grating sample. By dividing the measured absorption by the extrapolated one, one can eliminate the influence of the front surface reflection. In Figure 5.38 b), the resulting absorption curves for the reference, the linear grating and the crossed grating sample are shown. These absorption curves only take into account how much light was absorbed that entered the cell. 
a)

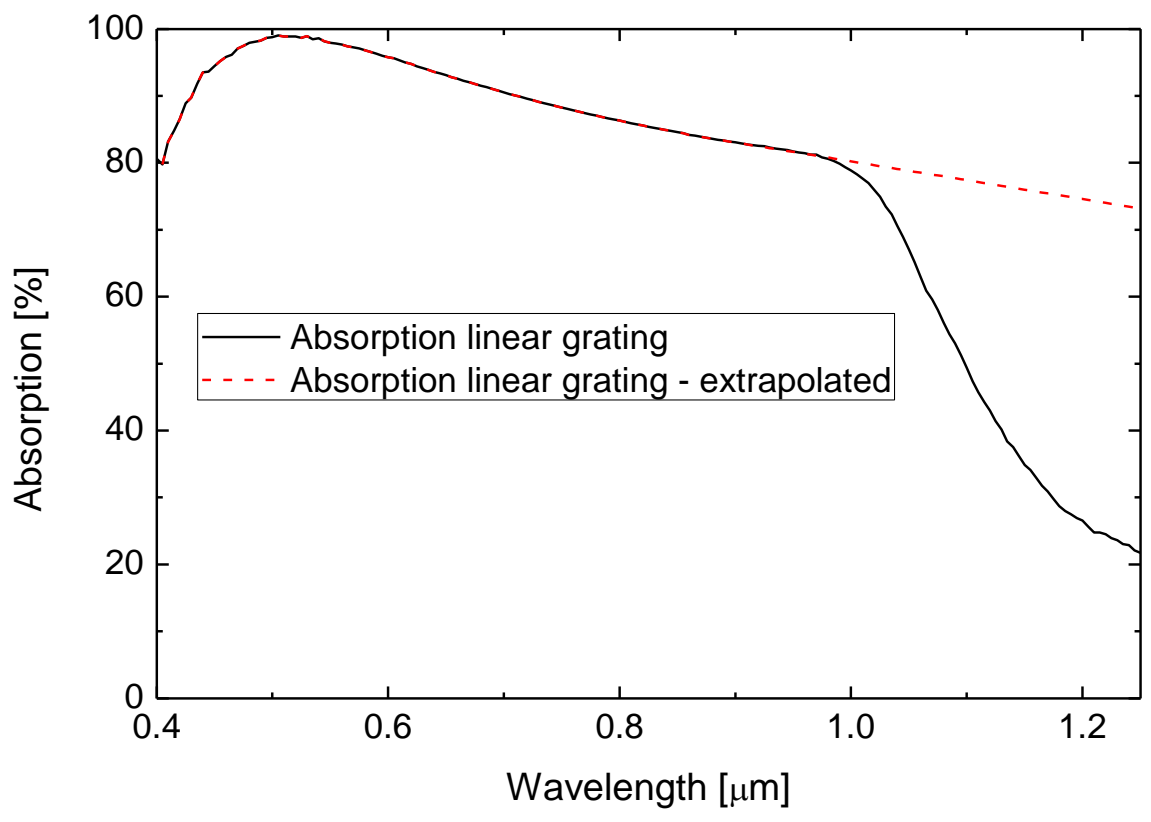

b)

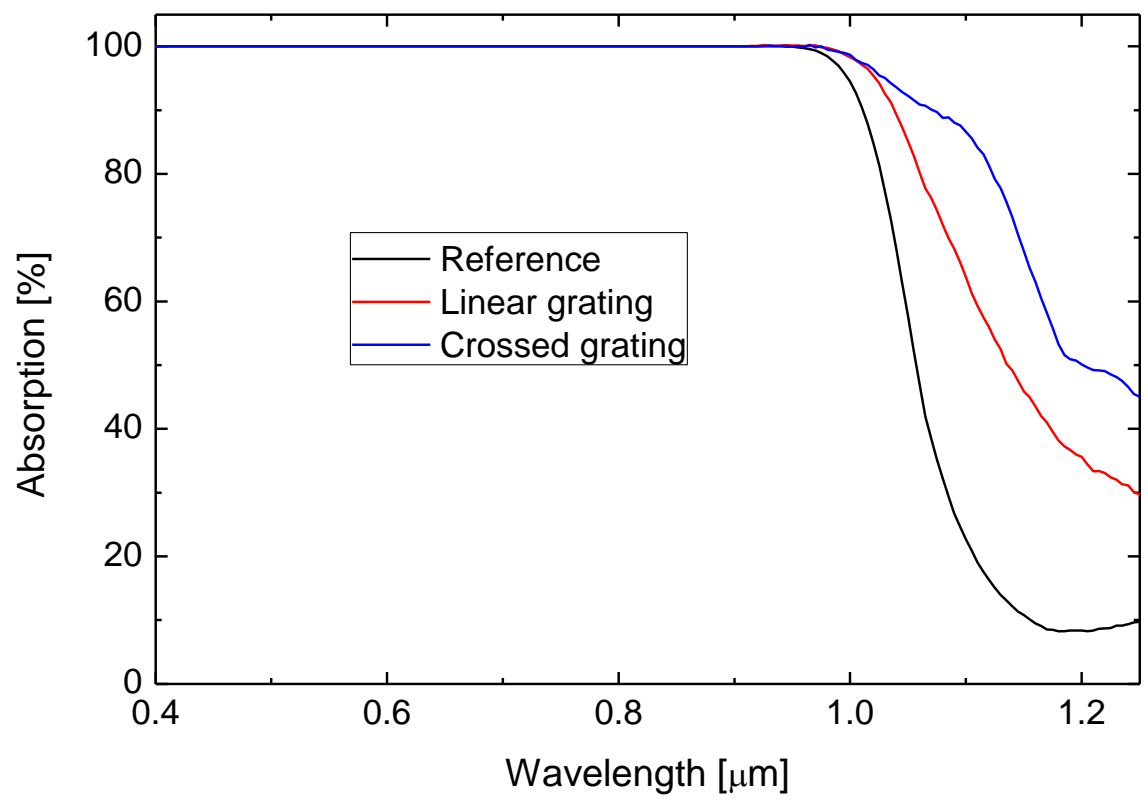

Figure 5.38: In a) the absorption measurement of a sample with a linear grating is shown. Additionally, this measurement is extrapolated linearly above $900 \mathrm{~nm}$ to visualise the absorption of an infinitively extended wafer. By dividing the measured by the extrapolated curve, the relative absorption of all light that entered the cell can be calculated. This is shown in b) for the planar reference as well as for samples with a linear and a crossed grating.

In a next step, the absorptivity of silicon is used to calculate necessary path lengths to achieve corresponding absorptions as shown in Figure 5.38 b). Then, the path 
lengths obtained for the grating samples were divided by those for the planar sample. The resulting graph is shown in Figure 5.39. It can be seen that the path length enhancement is high as about 10.2 for the crossed and 5.9 for the linear grating at around $1145 \mathrm{~nm}$ and $1170 \mathrm{~nm}$ respectively. However, it must be stated that the measured absorption enhancement also includes the parasitic absorption in the aluminium layer. Therefore, this path length enhancement factor has to be seen as an upper bound estimation.

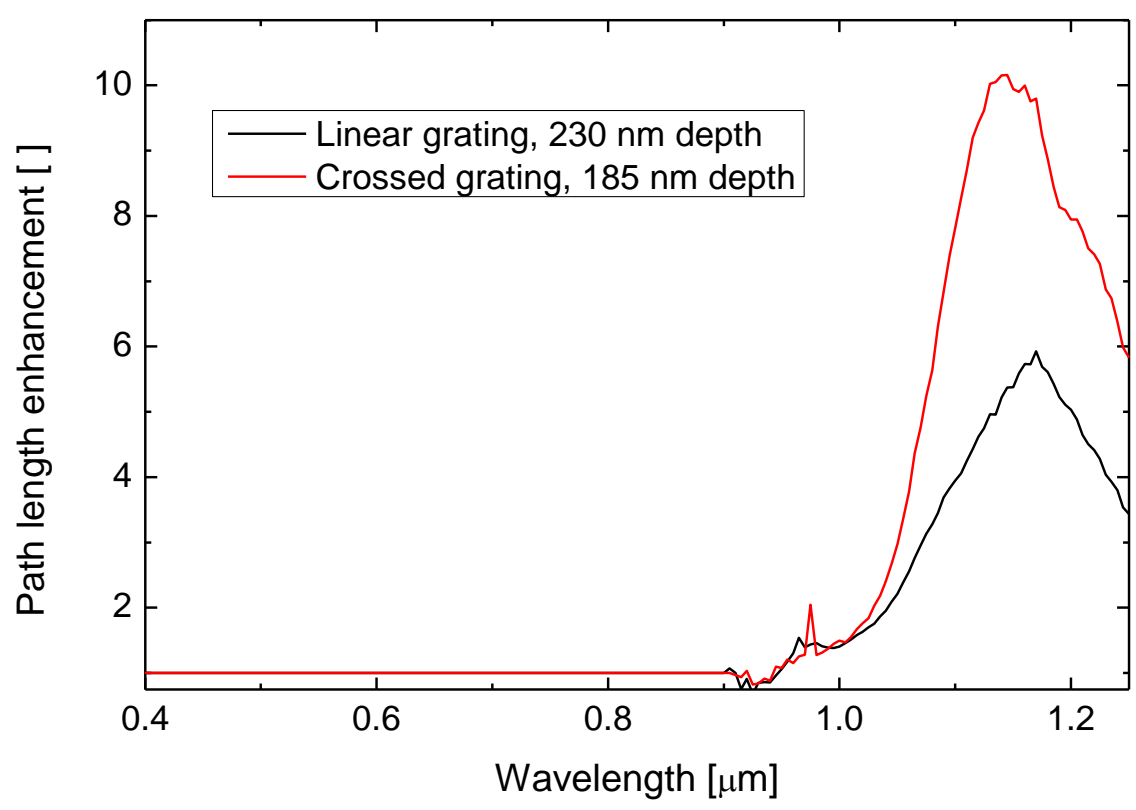

Figure 5.39: Factor of the path length enhancement introduced by rear side gratings calculated from the measured absorption enhancements shown in Figure 5.37 under the assumption that all absorption occurs in the silicon.

However, besides these positive figures that can be extracted, also considerable parasitic absorption is detected. This can be seen for wavelengths corresponding to photon energies lower than $E_{G a p}$. As silicon is transparent for wavelengths above $1200 \mathrm{~nm}$, it can be concluded that the absorption enhancement in this spectral range can be entirely attributed to absorption in the aluminium reflector. One possible effect leading to such a strong parasitic absorption in this metal layer is that it might not be planar as originally intended. This deduction is reinforced by polarisation-dependent reflection measurements for the linear grating samples. It can be observed that p-polarised light experiences a very pronounced absorption enhancement (see Figure 5.40). This polarisation-dependent parasitic absorption is typical of corrugated metal patterns as result of plasmonic effects [213, 214]. This non-usable absorption happens to occur not only in the energy range below $E_{G a p}$ 
of silicon, but also above. Therefore, it has to be expected that the values for $\Delta j_{p h}$ also include a considerable part of parasitic absorption.

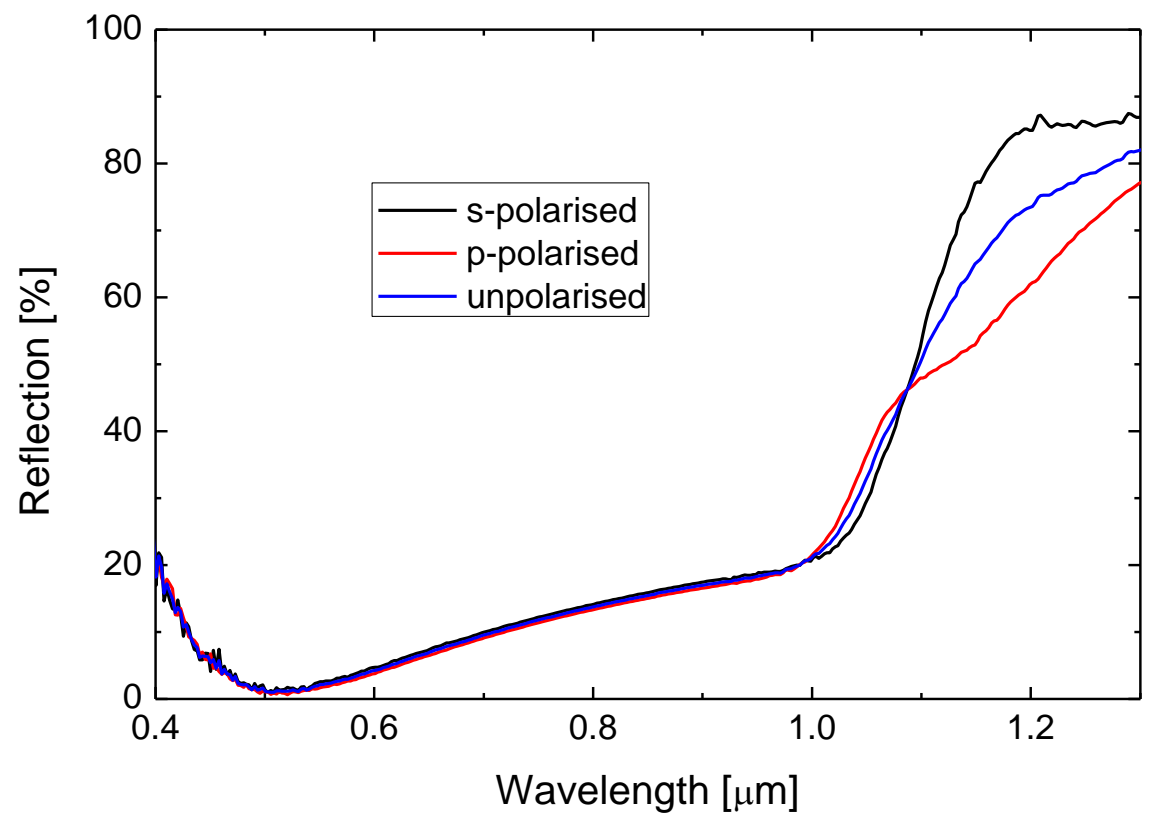

Figure 5.40: Polarisation-dependent reflection measurement of a sample with a linear grating of a depth of $230 \mathrm{~nm}$.

As expected by the polarisation-dependent reflection measurements, SEM images of these samples confirm that the PECVD deposited buffer layer has grown conformally with the grating leading to a modulated oxide-aluminium interface (see Figure 5.41). This is known to increase parasitic absorption in the reflector [64].

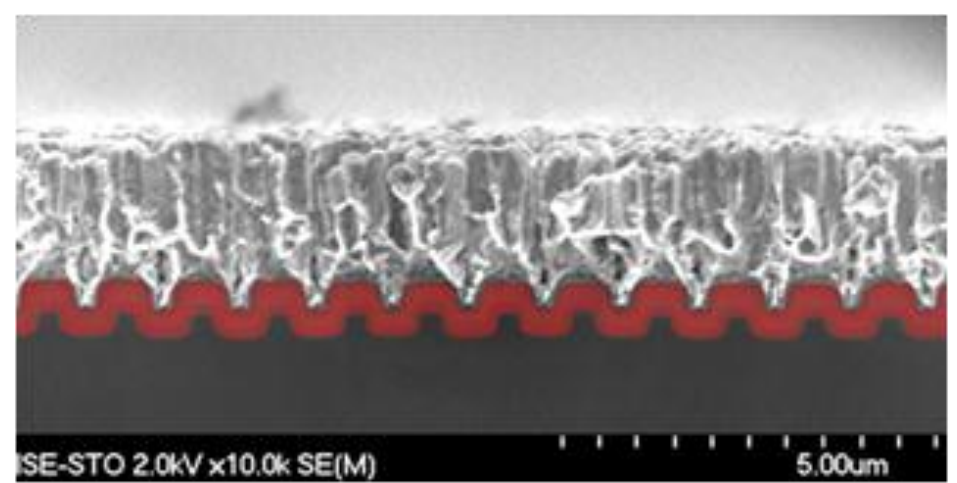

Figure 5.41: SEM image of the wafer rear side. The PECVD silicon oxide is dyed red to visualize the patterned aluminium back reflector (top). The depth of the etched grating is $230 \mathrm{~nm}$.

The useful absorption in silicon can be enhanced if parasitic absorption in the rear reflector is minimised. There are some strategies to minimise this unwanted absorption: 
(i) Increasing the $\mathrm{SiO}_{2}$ layer thickness, when applying PECVD to achieve a levelling of the rear interface. However, too thick $\mathrm{SiO}_{2}$ layers might cause problems later on, when it comes to the local contacting of solar cells.

(ii) Using a material of a lower refractive index as the dielectric buffer layer. Then, two positive effects can be expected: The non-lossy reflection at the interface silicon / dielectric as well as the damping of evanescent waves (which are likely to couple energy into the metal layer) in the dielectric are enhanced. However, the refractive index of $\mathrm{SiO}_{2}$ is already relatively low, so that dense layers will not improve this behaviour tremendously.

(iii) Applying other deposition processes for the dielectric buffer layer, so that its rear interface ends up planarised. This can be achieved for techniques based on the deposition of liquid phase materials or nanoparticle dispersions via spin, roller or dip coating processes.

Within this work, the latter approach of applying a $\mathrm{SiO}_{2}$ layer via spin coating of a nanoparticle dispersion was investigated experimentally. A commercially available dispersion of colloidal silica nanoparticles with a mean particle size of $7 \mathrm{~nm}$ diameter has been applied. The main solvent used for this dispersion is deionised water. In the laboratory scale, the most reproducible way to apply thin defined layers using liquid phase materials is spin coating. The task for developing a process to realise spin-coated $\mathrm{SiO}_{2}$ layers for the given purpose is to minimise topographic modulations of the silicon oxide surface. Other demands are that the layer thicknesses of this buffer layer shall be potentially tolerable for the laser contact firing in solar cells later on (in the range of up to $500 \mathrm{~nm}$ ) and cracks appearing during the drying of the dispersion shall be avoided. These cracks can occur due to shrinkage when driving out the solvent. By adding a small amount of tensides to the aqueous silica nanoparticle dispersion the formation of cracks was minimised. Thereby the surface energy of the solution was modified. Figure 5.42 shows $\mathrm{SEM}$ samples of spin-coated $\mathrm{SiO}_{2}$ layers on top of imprinted resist layers without and with adding tensides to the dispersion. 
a)

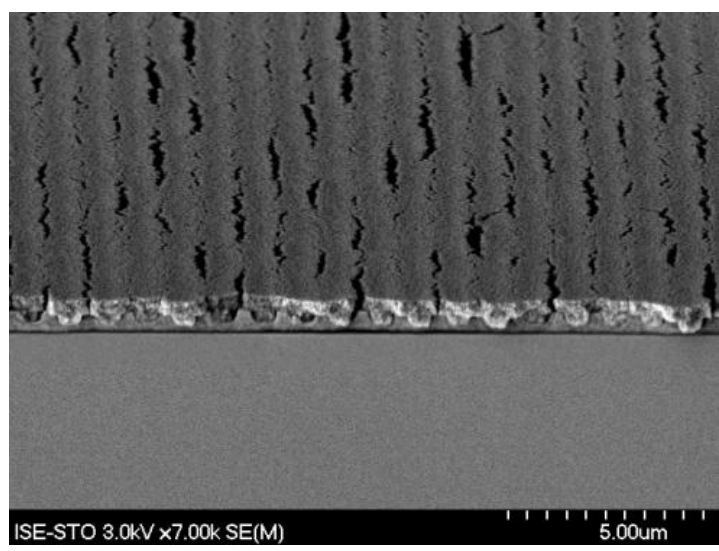

b)

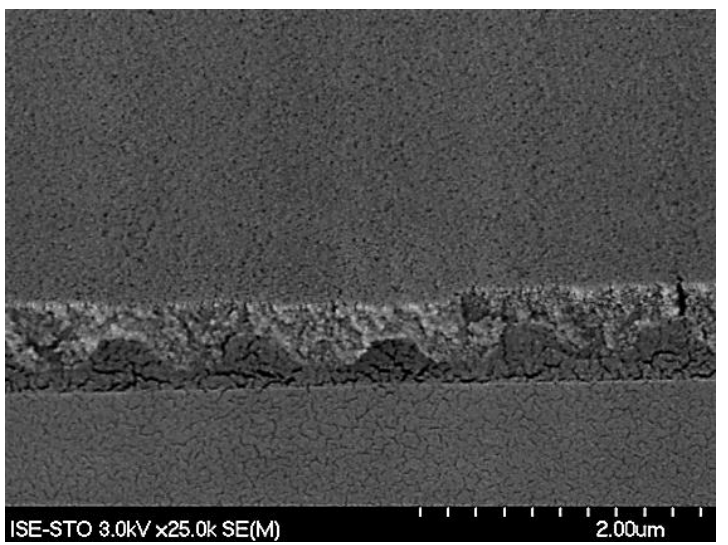

Figure 5.42: SEM micrographs of spin-coated silicon oxide layers on top of imprinted grating structures. a) shows cracks in this layer, which appeared during the drying of this layer. In b) a cleaning agent was added to the aqueous silica nanoparticle dispersion. Thereby a homogeneous layer of around $470 \mathrm{~nm}$ thickness with respect to the resist cavities was realized.

These spin-coated $\mathrm{SiO}_{2}$ layers were then integrated into solar cell precursors in the same way as for the PECVD coated buffer layers. Again the absorption enhancement was determined compared to a flat surface. Figure 5.43 shows a comparison of measured spectral absolute absorption enhancements for crossed gratings. One had a PECVD deposited and one a spin-coated $\mathrm{SiO}_{2}$ buffer layer.

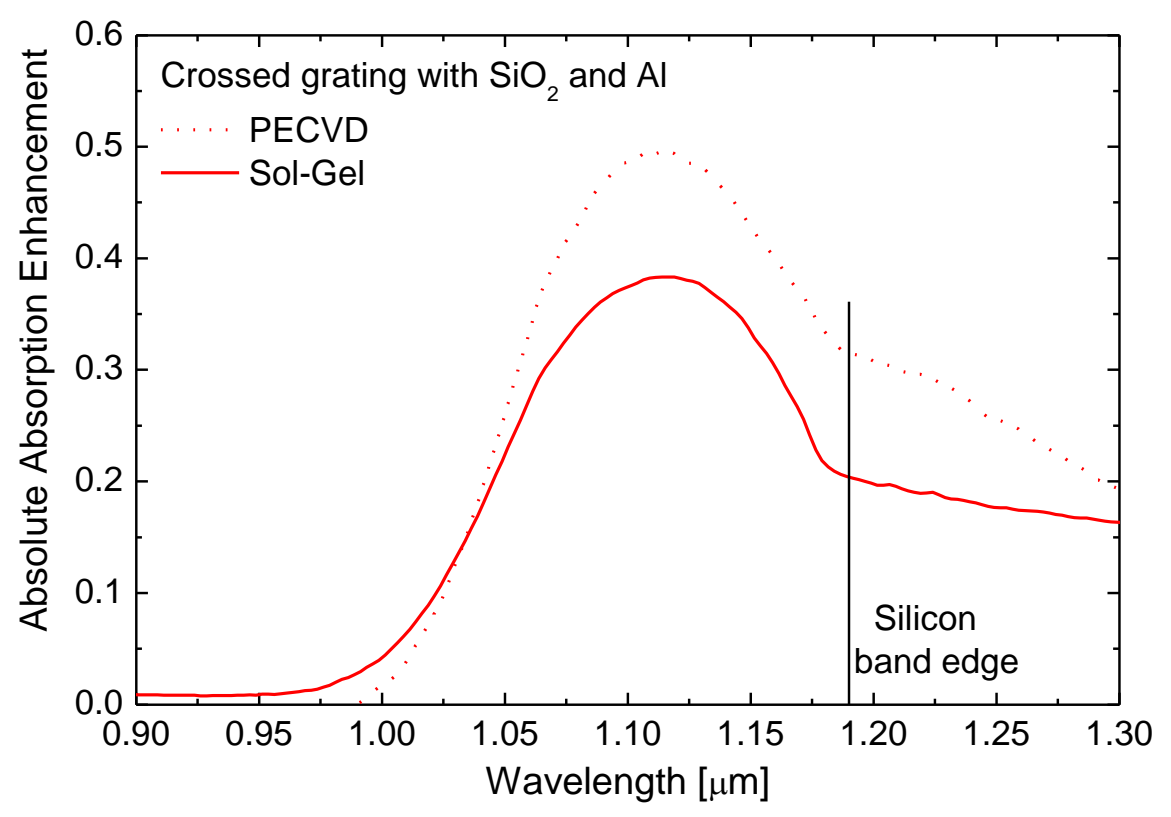

Figure 5.43: Comparison of absolute absorption enhancements induced by rear side diffraction gratings for different techniques to realise a silicon oxide buffer layer between the grating in silicon and the rear side aluminium reflector. 
This comparison shows that for photon energies below $E_{\text {Gap }}$ of silicon, less parasitic absorption occurs. Furthermore, the absorption enhancement is also diminished in the usable range below $1.18 \mu \mathrm{m}$. As the difference above and below $E_{G a p}$ is similar, it can be expected that the diminished part mainly corresponds to parasitic absorption. It has to be stated that not all samples showed this expected behaviour. Comparisons of linear gratings in some cases indicated that parasitic absorption might even be enhanced by applying this spin-coated layer. However, this also strongly depends on the buffer layer thicknesses (Fabry-Pérot effects) and thus reference samples behave totally differently. Simulations performed by A. Mellor outside of the frame of this thesis indicated that the desired effect of reducing parasitic absorption is achieved by the introduction of the spin-coated $\mathrm{SiO}_{2}$ layer $[215,216]$. Selected results of these simulations will be shown in the following section within the estimation of the potential for solar cell efficiency enhancement of these fabricated rear side gratings in combination with flat front surfaces. When calculating the path length enhancement as demonstrated before, the maximum factor of 10.2 is reduced to 6.9 at a wavelength of around $1.15 \mu \mathrm{m}$ for crossed gratings and the spin-coated dielectric buffer layer. This indicates further that these values calculated before overestimate the actual gain.

When investigating these solar cell precursors in the SEM, it was found that although the spin-coated $\mathrm{SiO}_{2}$ layer was initially planar, after the evaporation of aluminium it shows a slight modulation (see Figure 5.44). This modulation is likely to be the result of a densification of the nano-porous network of silica particles due to the temperature treatment during the thermal evaporation of the aluminium. In the tool used for the evaporation of $2 \mu \mathrm{m}$ of aluminium, surface temperatures around $200^{\circ} \mathrm{C}$ were measured. To evaluate the densification of the silicon oxide layer, planar silicon wafers were spin-coated. These samples were measured using ellipsometry before and after a temperature treatment simulating the evaporation process (on a hotplate). As a result of this temperature exposure, both a slight decrease in film thickness as well as an increase in the refractive index was detected. This strengthens the assumption that the modulation of the aluminium layer even for the spin coating deposition of silicon oxide is related to a densification. 


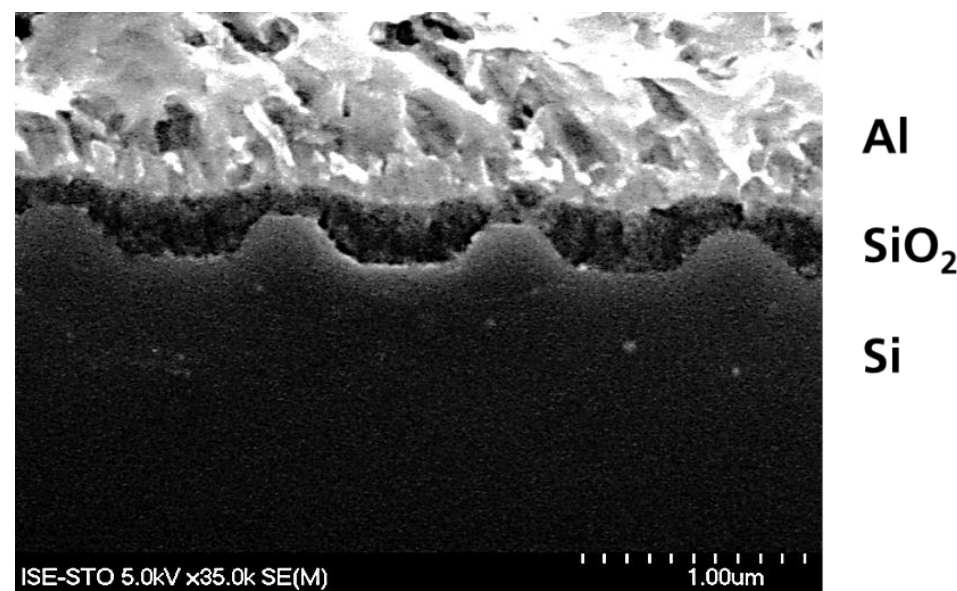

Figure 5.44: SEM micrograph of rear side layer stack of silicon with etched grating - spin-coated $\mathrm{SiO}_{2}$ layer - and evaporated aluminium.

\section{Electrical characterisation}

The patterning of solar cell surfaces implies increasing the effective surface recombination velocity $S$. This is particularly crucial for nanopatterned or porous surfaces as described in section 3.3. In a very first study, we investigated the direct etching of a grating into the silicon bulk which was then passivated by a PECVD silicon nitride. Lifetime measurements revealed low values of below $20 \mu \mathrm{s}$ for high quality $p$-type FZ material. Thus, the electrical quality was deteriorated crucially. Therefore, as mentioned before, we aim for a concept where the optically active patterned surface is decoupled from a passivated planar solar cell rear surface [217]. In principle, the most elegant way to realise this would be to have a rear surface passivation made of amorphous silicon (a-Si). Thereby, very low surface recombination velocities at the interface silicon/a-Si can be achieved [51]. However, the passivation quality of a-Si can be strongly deteriorated by thermal treatments. Therefore, aluminium oxide was chosen as material for surface passivation. This layer has an excellent passivation quality and very thin layers can be realised via atomic layer deposition (ALD) [218]. Onto this very thin passivation layer, an a-Si layer was deposited, which was then patterned via NIL and plasma etching. Within the present study, the passivation quality of this layer stack was investigated before and after the texturisation sequence.

The sample preparation was done using $250 \mu \mathrm{m}$ thick $p$-type FZ $1 \Omega \mathrm{cm}$ silicon material with $\langle 100\rangle$ orientation. The samples were symmetrically (front and rear) passivated by $10 \mathrm{~nm} \mathrm{Al}_{2} \mathrm{O}_{3}$ deposited via plasma enhanced ALD. Then both sides were coated with $300 \mathrm{~nm}$ a-Si applied via PECVD. After a forming gas anneal (25 minutes at $425^{\circ} \mathrm{C}$ ), initial lifetimes were measured via QssPC to extract values for $S$. The following processing scheme for the texturisation comprises of the process 
steps of spin coating of the resist, NIL, RIE and resist removal. These processes were only conducted on a single side. For the resist removal, the two approaches of plasma ashing as well as a wet-chemical removal based on the dissolution of a sacrificial layer were investigated. The objective was to maintain high values for $S$ throughout the texturisation. A series of experiments was conducted to isolate the influences of the distinct processes on the lifetime after texturisation and thus $S$ according to Figure 5.45 .

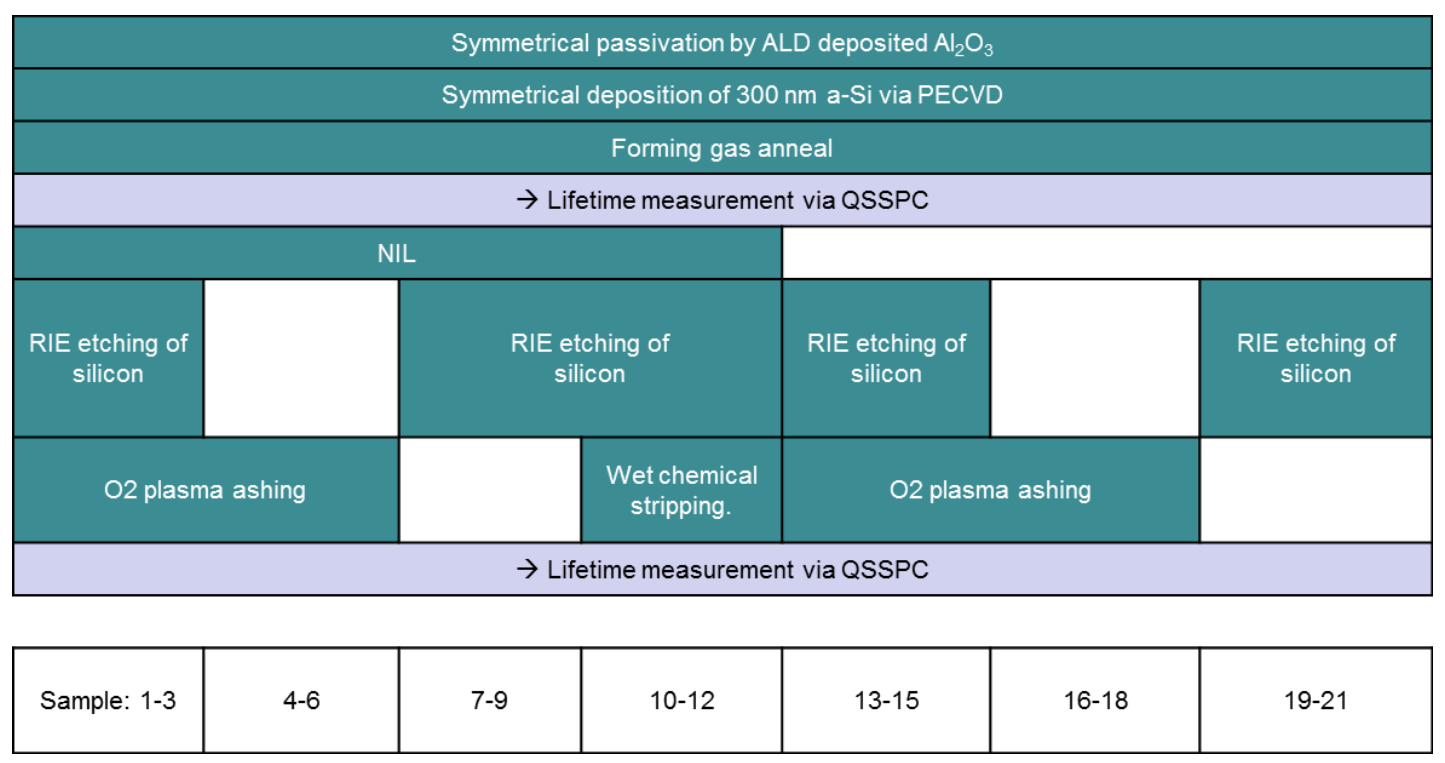

Figure 5.45: Overview of the design of experiments to investigate the influence of processes for grating fabrication on minority carrier lifetimes.

Figure 5.46 shows the results of this study in the form of measured lifetimes before and after the treatment with the listed process variations. As shown in Figure 5.46, for all configurations three samples were processed. The lifetime measurement for each sample was conducted with the processed surface face up as well as face down. The values shown represent the averages of these two measurements averaged over each group. It can be seen that there is already a variation in lifetimes in the as-deposited (or actually as-annealed) state between $1150 \mu \mathrm{s}$ and $1800 \mu \mathrm{s}$. The measurements after the processing vary between $1350 \mu \mathrm{s}$ and $1930 \mu \mathrm{s}$. As all changes in lifetimes after processing are lower than the deviation of the as-deposited layers, it can be concluded that there is no influence from the texturing processes and all samples are on a very good level. 


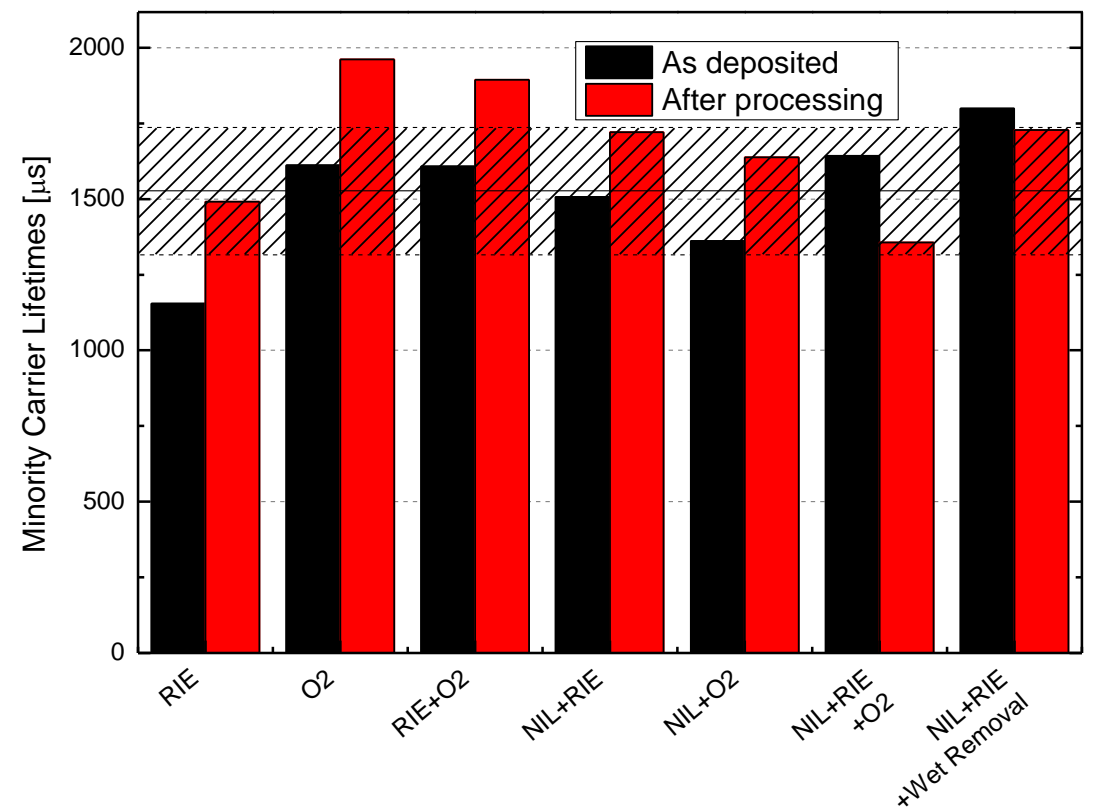

Figure 5.46: Lifetime measurements at an injection density of $10^{15} \mathrm{~cm}^{-3}$ of symmetrically passivated samples before and after specific treatments. On the $x$-axis the corresponding treatment is described. The hatched area visualises the standard deviation in the as-deposited lifetimes.

Values for $S$ were extracted from these lifetime measurements. First, in the initial state a symmetrical setup was assumed. In the as-processed state, the untreated surface was assumed to be unchanged and any change was assumed to be introduced by the $S$ of the processed surface. Figure 5.47 shows the resulting values for $S$ of the processed side. Again, the same statements formulated for the lifetime measurements can be made. There is not a distinct identifiable trend and all values are in an excellent range of $10 \mathrm{~cm} / \mathrm{s}$ and below. These results confirm that the concept of decoupling the electrical effects from the formation of an optically active grating can successfully be applied.

In the following, for the estimation of the efficiency potential of this rear side cell structure, the values for the $S_{\text {back }}$ as obtained for the texturing process chain made of the steps of NIL, RIE and oxygen plasma removal of the resist will be considered. There, values for the passivated samples $S_{\text {pass }}$ were measured to be $7.9 \mathrm{~cm} / \mathrm{s}$ and $10.8 \mathrm{~cm} / \mathrm{s}$ for the initially planar state and the texturised state afterwards. These values are both higher than the ones obtained for the wet-chemical resist removal and thus can be seen as the worst case (especially, when considering all other measurements, as they indicate that there is no influence of the patterning at all). 


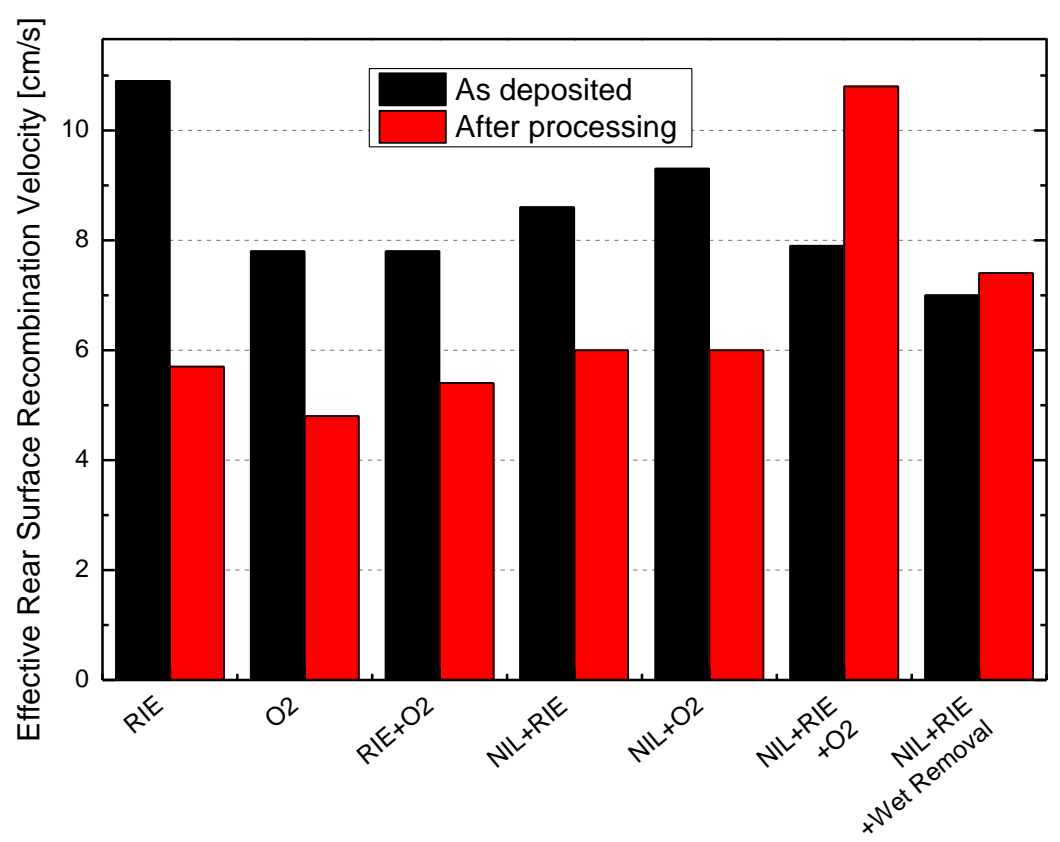

Figure 5.47: Values of surface recombination velocity extracted from the lifetime measurements of symmetrically passivated samples before and after specific treatments. On the $x$-axis the corresponding treatment is described.

\subsubsection{Estimation of the solar cell efficiency potential}

In the section before, the experimental studies on the optical and electrical properties of samples featuring rear side diffraction gratings were investigated. Now, these results will be used to estimate solar cell efficiencies incorporating such photonic structures at the wafer rear [216].

\section{Description of the optical model}

First, the optical measurements shown above were further analysed and compared to wave optical simulations by A. Mellor performed for the grating geometries discussed earlier $[215,216]$. By these simulations, he could distinguish between useful absorption in the silicon bulk and parasitic absorption in the aluminium layer. A flat front surface coated by a $\operatorname{SiN}_{x} A R C$ was assumed for these simulations. The selected geometries are based on the ones obtained for the deposition of the PECVD and the spin-coated silicon oxide buffer layer. The corresponding samples are listed in Table 5.7. 
Table 5.7: Grating type and deposition technique for the silicon oxide layer for each sample.

\begin{tabular}{lll}
\hline \hline Sample name & Grating type & Dielectric buffer layer via \\
A & crossed grating & PECVD \\
B & linear grating & PECVD \\
C & linear grating & Spin Coating \\
Ref & no grating & PECVD \\
\hline \hline
\end{tabular}

Figure 5.48 shows the investigated geometries of the grating structure on the solar cell rear as well as results of the optical simulations compared to the measurements.
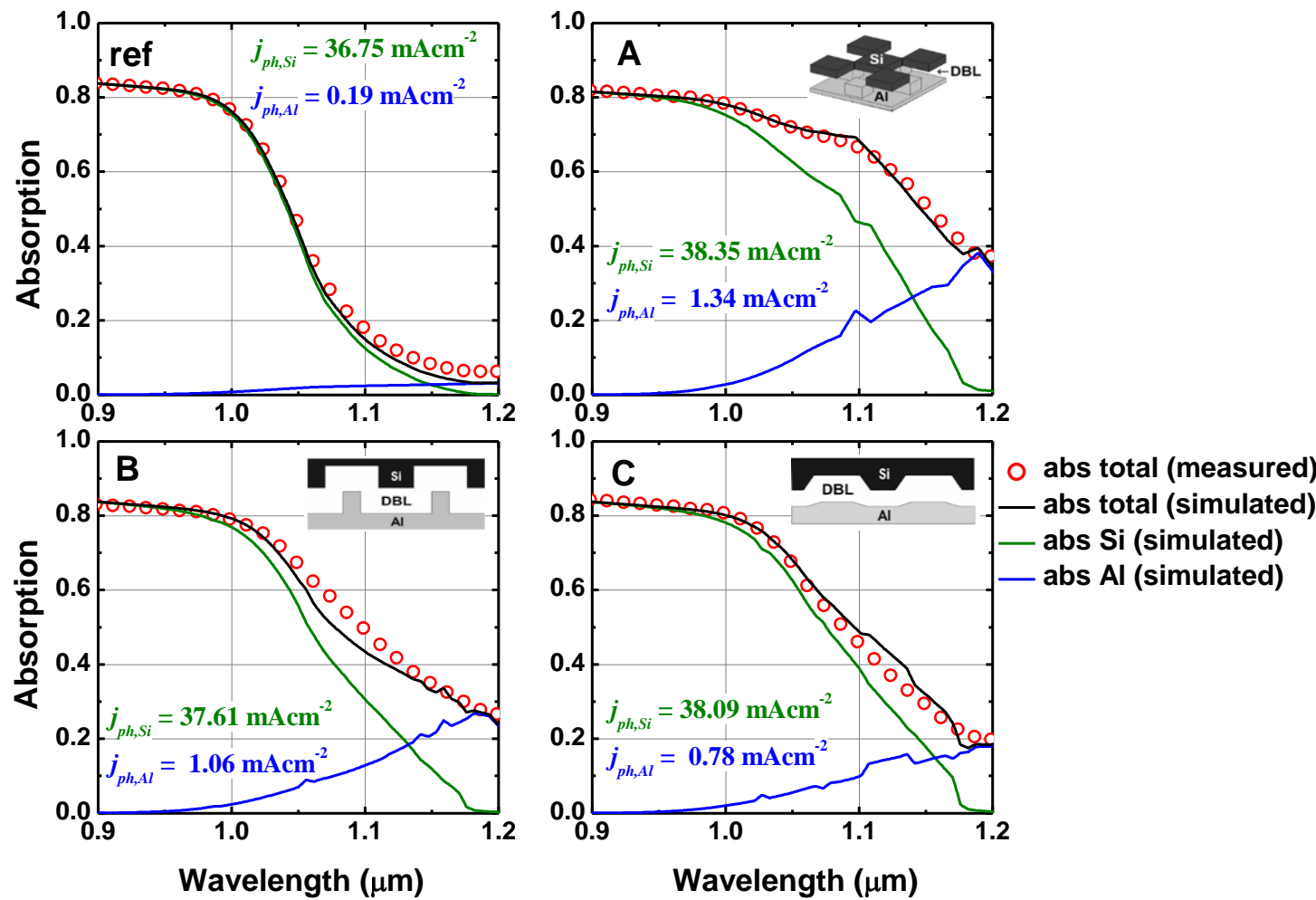

Figure 5.48: Results of wave optical simulations concerning the absorption behaviour of different rear side setups compared to measured values. The reference (ref) has a planar rear. For samples A$C$, there are sketches within the graphs. The simulated absorption data is separated into absorption in the silicon bulk as well as in the aluminium reflector [216].

Case A corresponds to the corrugated aluminium surface as obtained for the PECVD deposition of silicon oxide on a crossed grating. Case B and C correspond to the modulated surfaces after PECVD or spin coating of the nanoparticle dispersion and subsequent evaporation of aluminium on a linear grating respectively. Additionally, a planar reference was also simulated. As can be seen, these simulations are in very good agreement with the measured values for the 
overall absorption. The splitting in useful and parasitic absorption allows a more profound estimation of the efficiency potential of these structures. The excellent agreement between optical measurements and simulation is further confirmed by reviewing the polarisation-dependent measurement already shown before in Figure 5.40. In Figure 5.49 now additionally the simulated data is plotted.

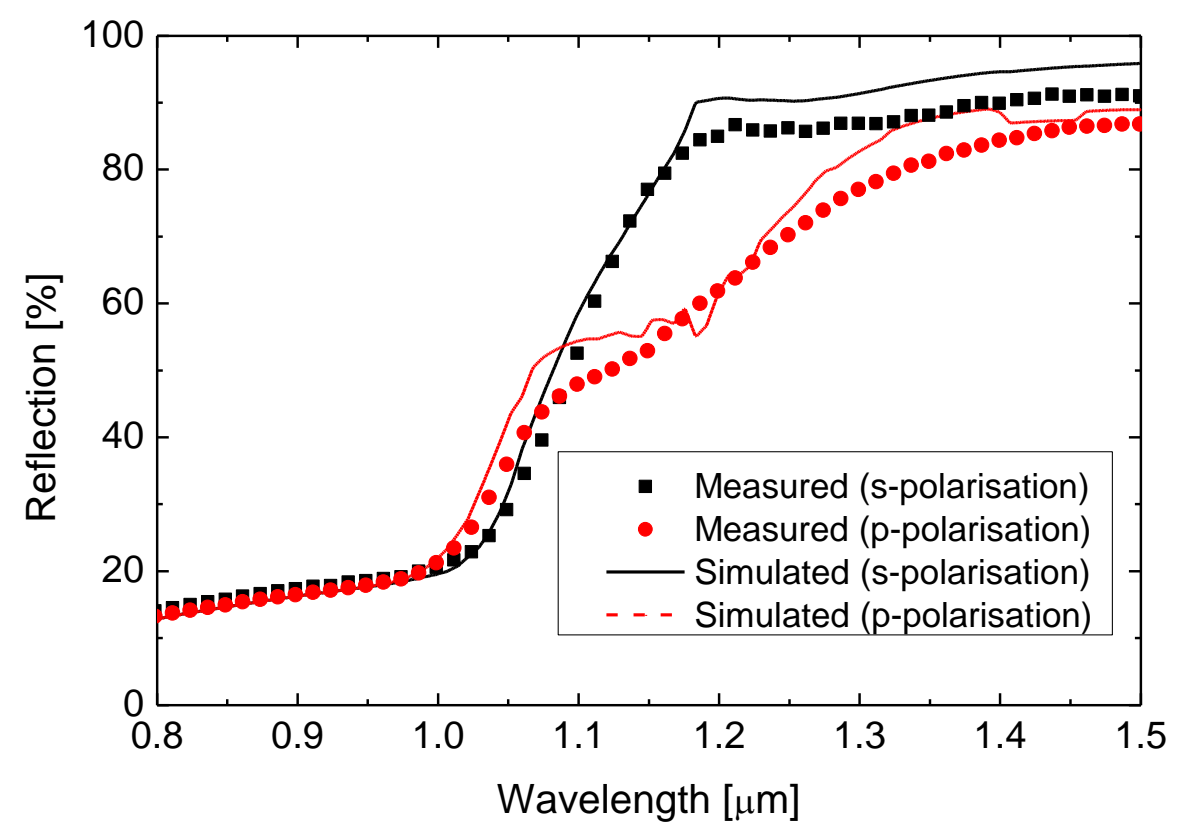

Figure 5.49: Polarisation-dependent reflection measurement and simulation of a sample with a linear grating and PECVD dielectric buffer layer.

Having gained confidence in the optical simulation method, this tool can now be used to generate spectral photo-generation data in dependence of the wafer depth, which can be used as input data in an electrical one-dimensional solar cell simulation using PC1D [13]. Conceptually dividing the silicon wafer into depth intervals, the optical simulation can be used to calculate how much absorption occurs in each interval at each wavelength. These values are integrated over the AM1.5g spectrum and tabulated cumulatively with respect to depth. Cumulatively means here that we tabulate the total number of photons absorbed in depth intervals of $0-10 \mu \mathrm{m}, 0-20 \mu \mathrm{m}, 0-30 \mu \mathrm{m}$ etc. Figure 5.50 exemplarily shows such photo-generation profiles for the planar reference as well as a sample with a grating (Case B). This simulation approach can be applied to calculate the influence of the practically realisable grating structures in very thin solar cells. To this end, photo-generation profiles for $40 \mu \mathrm{m}$ as well as $200 \mu \mathrm{m}$ thick silicon solar cells with the same rear side setups were calculated. 


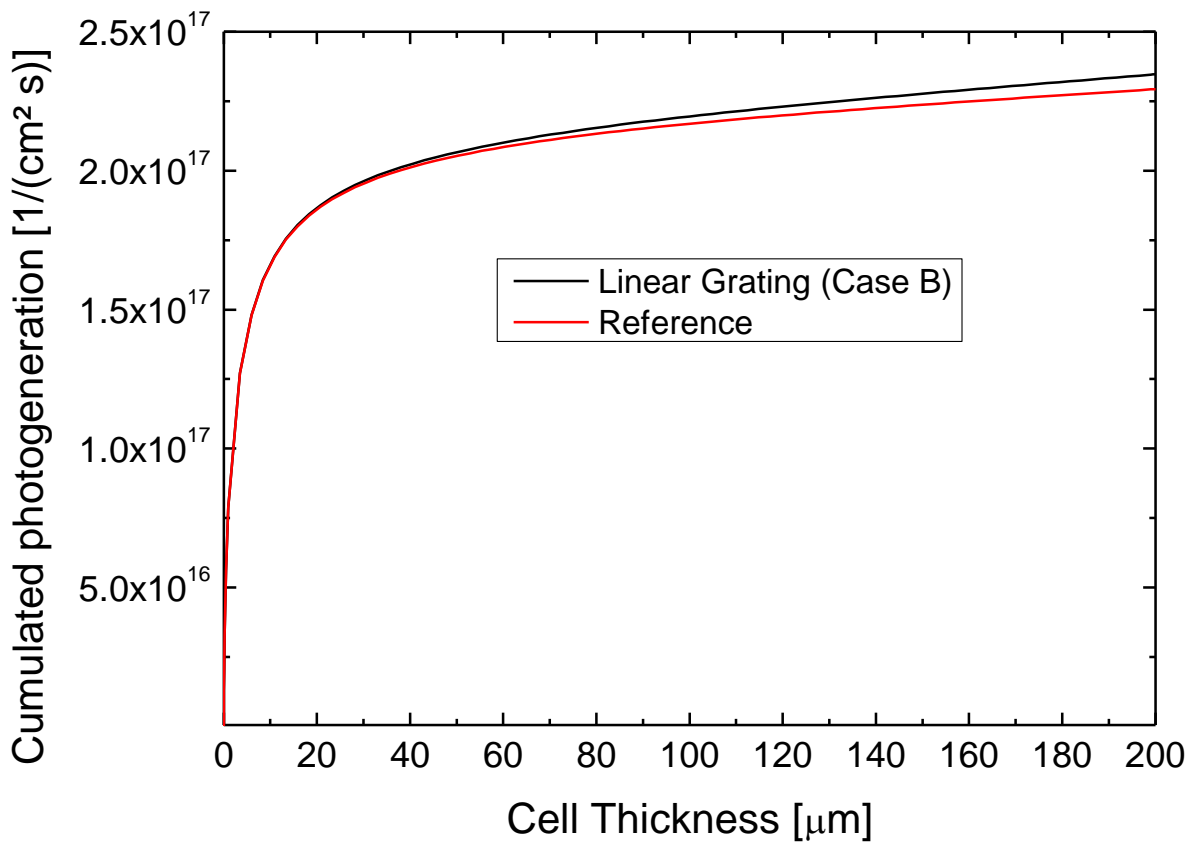

Figure 5.50: Cumulated photo-generation for a $200 \mu \mathrm{m}$ thick planar reference and a sample with a rear side grating. Cumulating in this case means both integrating over the $A M 1.5 \mathrm{~g}$ spectrum as well as cumulating over the depth from $0 \mu \mathrm{m}$ to the value shown on the axis.

\section{Description of the electrical model}

Having determined the optical input parameters, the electrical parameters of a solar cell model have to be defined. It is important to consider the architecture of the solar cell precursors investigated before, so that the corresponding measurements of $S_{\text {pass }}$ can be applied within this model. Therefore, here a solar cell made of high-efficiency $p$-type FZ $1 \Omega \mathrm{cm}$ material will again be considered. The bulk lifetime is assumed to be $1 \mathrm{~ms}$. The flat front has a diffused phosphorous emitter with Gaussian shape, $1 \cdot 10^{19} \mathrm{~cm}^{-3}$ peak doping and a junction depth of $1 \mu \mathrm{m}$. For the rear surface, a contacting based on the laser-fired-contacts (LFC) approach is assumed. Thus, an effective rear surface recombination velocity $S_{\text {eff }}$ can be calculated using the Fischer's formula as derived in [219].

$$
\begin{aligned}
S_{\text {eff }}=\frac{D}{W}\left(\frac{L_{p}}{2 W \sqrt{\pi f}} \operatorname{ArcTan}\left(\frac{2 W}{L_{p}} \sqrt{\frac{\pi}{f}}\right)\right. \\
\left.-\exp \left(-\frac{W}{L_{p}}\right)+\frac{D}{f W S_{m e t}}\right)^{-1}+\frac{S_{\text {pass }}}{1-f}
\end{aligned}
$$


$D$ stands for the diffusion constant of minority carriers, $W$ is the wafer thickness, $L_{p}$ the contact pitch, $f$ the fractional area that is metallised and $S_{m e t}$ and $S_{\text {pass }}$ the $S R V$ of the metallised and passivated areas of the wafer rear side respectively.

The parameters to determine $S_{\text {eff }}$ in dependency of $S_{\text {pass }}$ were based on those published in [220]. Critical parameters for this model are the pitch and the radius of the local contacts as well as the value of $S_{\text {met }}$. In [220] a pitch of around $800 \mu \mathrm{m}$ was found to be the optimum for $200 \mu \mathrm{m}$ thick $p$-type $1 \Omega \mathrm{cm}$ material. There, a radius of $45 \mu \mathrm{m}$ of the metal contacts was considered leading to a metal fraction of about $1 \%$.

An effective $S_{m e t}$ was determined using the empirical model introduced in [197]

$$
S_{m e t}=S_{0}+\alpha e^{\beta\left(N_{A}+N_{0}\right)},
$$

with $S_{0}=-900 \mathrm{~cm} / \mathrm{s}, \alpha=22.1 \mathrm{~cm} / \mathrm{s}, \beta=1.29 \cdot 10^{-16} \mathrm{~cm}^{3}, N_{A}$ being the base doping of $1.53 \cdot 10^{16} \mathrm{~cm}^{-3}$ and $N_{0}=3.4 \cdot 10^{16} \mathrm{~cm}^{-3}$. This leads to an $S_{\text {met }}$ of $11.9 \cdot 10^{3} \mathrm{~cm} / \mathrm{s}$. As a result of the Fischer equation, values for $S_{\text {eff }}$ are $60 \mathrm{~cm} / \mathrm{s}$ and $64 \mathrm{~cm} / \mathrm{s}$ for the planar and for the textured rear side respectively. Additionally, solar cells with a thickness of $40 \mu \mathrm{m}$ were also simulated. For these, slightly higher values for $S_{\text {eff }}$ of $69 \mathrm{~cm} / \mathrm{s}$ and $72 \mathrm{~cm} / \mathrm{s}$ were obtained. The Fischer equation again was applied and the pitch was not changed, which is probably not fully correct and thus this can only serve as a rough estimation for these thin cells. $S_{\text {front }}$ was chosen to $1000 \mathrm{~cm} / \mathrm{s}$ according to [221] (omitting the influence of front contacts) and shading losses on the front surface have not been considered within this study. The resulting IV-parameters of the PC1D simulations based on the solar cell characteristics described above are shown in Table 5.8. The efficiencies have to be seen as upper limits for the given $j_{s c}$ and $V_{o c}$ values as ideal cell parameters for series and parallel resistances were assumed leading to an ideal fill factor of around $84 \%$. The values given for the photocurrent density $j_{p h}$ show the amount of useful absorption in the silicon obtained out of the optical simulations. 
Table 5.8: Results of the PC1D simulations for the calculated photogeneration files both for $200 \mu \mathrm{m}$ as well as $40 \mu \mathrm{m}$ thick cells with a planar front and a single layer ARC. The efficiencies listed result of an ideal fill factor of around $84 \%$ (no additional series resistances in the metallisation). Nomenclature of sample names: ref: planar rear; A: crossed grating with PECVD silicon oxide; B: linear grating with PECVD silicon oxide; C: linear grating with spin-coated silicon oxide.

\begin{tabular}{|c|c|c|c|c|}
\hline $\begin{array}{l}\text { Structure } \\
\text { name }\end{array}$ & $\begin{array}{c}j_{\mathrm{sc}} \\
{\left[\mathrm{mA} / \mathrm{cm}^{2}\right]}\end{array}$ & $\begin{array}{c}V_{o c} \\
{[\mathrm{mV}]}\end{array}$ & $\begin{array}{c}\eta \\
{[\%]}\end{array}$ & $\begin{array}{c}j_{p h} \\
{\left[\mathrm{~mA} / \mathrm{cm}^{2}\right]}\end{array}$ \\
\hline \multicolumn{5}{|c|}{$200 \mu \mathrm{m}$ thick cells } \\
\hline ref & 36.5 & 685.8 & 21.1 & 36.7 \\
\hline$A$ & 38.0 & 686.1 & 22.0 & 38.3 \\
\hline B & 37.3 & 685.7 & 21.6 & 37.6 \\
\hline C & 37.8 & 685.9 & 21.9 & 38.0 \\
\hline \multicolumn{5}{|c|}{$40 \mu \mathrm{m}$ thick cells } \\
\hline ref & 33.7 & 686.2 & 19.5 & 33.8 \\
\hline$A$ & 36.4 & 687.2 & 21.1 & 36.5 \\
\hline B & 35.0 & 686.4 & 20.3 & 35.1 \\
\hline C & 35.9 & 686.9 & 20.8 & 36.0 \\
\hline
\end{tabular}

These results show clearly that, for the assumptions made concerning the contacting, no significant change in $V_{o c}$ is to be expected when applying the present concept for the integration of a photonic rear side structure. Furthermore, it is shown that the considerable optical absorption enhancement leads to a significant gain in $j_{s c}$, which is responsible for the efficiency enhancement of the simulated cells. As was already visible for the optical measurements of the absorption enhancement, also at cell level the bi-periodic gratings behave superior to the uni-periodic ones in accordance to the theoretical considerations published in [95]. For crossed gratings, the highest gain in efficiency can be achieved both for the $200 \mu \mathrm{m}$ as well as the $40 \mu \mathrm{m}$ thick cells, which are $0.9 \%$ and $1.6 \%$ absolute, respectively.

\subsubsection{Summary and next steps}

The results achieved up to now show that there is a considerable absorption enhancement by applying the rear side grating even for thick wafer based silicon solar cells. Furthermore, it was shown that the fabrication process of these photonic structures at the wafer rear does not necessarily go in hand with deteriorating the electrical quality of silicon solar cells. However, the practical proof of these results at cell level has to be realised in the future to verify these preliminary results.

To this end, currently on-going work is related to the local contact formation on the rear side via laser-fired-contacts (LFC) or laser ablation with subsequent evaporation of aluminium. This work is necessary as standard types of PERC solar 
cells typically have a thin (around $100 \mathrm{~nm}$ ) silicon oxide or silicon nitride layer, through which an aluminium layer has to be fired using laser pulses. For the concept described above, there is a $10 \mathrm{~nm}$ aluminium oxide layer, the structured amorphous silicon layer (initially around $300 \mathrm{~nm}$ thick, after the etching the residual layer is around $50 \mathrm{~nm}$ thick) and the silicon oxide buffer layer, which is around $400 \mathrm{~nm}$ thick. Test structures have to be fabricated to investigate laser parameters (pulse energy, duration, wavelength, number of pulses...) to achieve adequate contact resistances.

In parallel to the work on the LFC process, there is also currently a solar cell batch in preparation. This will be a $p$-type PERT cell structure, in which the passivation stacks investigated in this work can be applied. Within this solar cell batch, the combination of pyramidal front side textures with the diffractive gratings on the rear will be investigated also. This will reduce the gain introduced by the gratings and the question will be how much gain will be left.

Besides these issues, another interesting question is if there are possibilities to leave out classical front side textures and introduce other features to obtain effective antireflection properties. All light trapping or path length enhancement in this case would solely be introduced by the photonic structure on the wafer rear. This might be possible by using multilayer antireflection coatings [222] or effective graded refractive index layers $[58,223]$. However, it will be difficult to beat random pyramid textures in terms of the achievable front surface reflection. Therefore, the solar cell architecture would probably have to benefit from e.g. a reduced emitter dark saturation current density due to the planar surface. This would allow higher values for $V_{o c}$ and thus slight increases in the front surface reflectivity might be tolerable.

In terms of optimising the light trapping performance using rear side diffraction gratings, there is still room for further optimisation. The benefit of the photonic structure is closely linked to the optical quality of the rear reflector. There, topographical modulations lead to increased parasitic absorption. Furthermore, the diffraction efficiencies are related to the contrast of the refractive indices of the involved media. These were amorphous silicon $(n \sim 3.5)$ and silicon oxide $(n \sim 1.5)$ as dielectric buffer layer within this work. Both of these optical effects can be optimised by a method published in [224] and patented in [225]. There, an aluminium foil is attached to the wafer rear side by laser firing of local contacts through the foil. Thereby, a very planar metal reflector can be realised even for a structured rear. What's more, that the silicon oxide dielectric buffer layer could be left out and an air gap could be realised between the a-Si grating and the planar metal reflector. This would lead to an increased refractive index contrast and thus the non-lossy reflection at the interface a-Si / dielectric buffer layer would be 
enhanced. As said before, the diffraction efficiencies would also be enhanced as a result of the enlarged refractive index contrast. A patent concerning improving the optical properties, in particular also in combination with a photonic rear side structure, was filed within the framework of this thesis [226]. 


\section{Conclusions}

The technology of nanoimprint lithography (NIL) offers completely new possibilities in terms of achievable pattern resolutions for photovoltaic applications. However, existing tools and processes were mostly aiming for microelectronic or biological sensing applications. Therefore, the aim of this thesis was the development of tools and processes adapted to the requirements in photovoltaics. The concrete application of NIL was the defined texturisation of wafer based silicon solar cells via subsequent plasma etching processes.

Within this thesis, a Roller-NIL tool was designed and constructed to combine the high-resolution capability of NIL technology with in-line feasible processing. Using soft stamps and UV-curing resist materials, this tool was proven to be able to pattern of etching masks on large areas of up to $156 \times 156 \mathrm{~mm}^{2}$. What is especially remarkable is that this is possible on very thin (tested down to $100 \mu \mathrm{m}$ ), opaque, brittle and also rough as-cut multicrystalline silicon substrates.

Two exemplary applications were investigated to verify the high potential of this novel texturing sequence based on NIL and plasma etching. These were the honeycomb texturing for multicrystalline silicon solar cells and the origination of diffractive rear side gratings as photon management structures, which are of particular interest for forthcoming thinner wafers.

The honeycomb texturing of the front side of multicrystalline silicon via NIL leads to excellent optical properties of around $14 \%$ weighted reflectance (without $A R C)$. This very high optical quality was confirmed on small area $\left(2 \times 2 \mathrm{~cm}^{2}\right) \mathrm{FZ}$ silicon solar cells by very high short-circuit current densities $j_{s c}$ of $40.1 \mathrm{~mA} / \mathrm{cm}^{2}$ and $40.7 \mathrm{~mA} / \mathrm{cm}^{2}$ for a single and a double layer ARC respectively. The up-scaling capability of the developed texturing process chain were demonstrated on $125 \times 125 \mathrm{~mm}^{2}$ wafers.

As a second application, diffractive rear side gratings were and their considerable absorption enhancement was demonstrated. Both linear and crossed gratings with a period of $1 \mu \mathrm{m}$ were realised. For the crossed gratings, absolute absorption enhancements of up to $52 \%$ absolute were measured at a wavelength of around $1100 \mathrm{~nm}$ for $200 \mu \mathrm{m}$ thick wafers. In this context a concept for minimising parasitic absorption by applying a dielectric buffer layer in the liquid phase was introduced. Furthermore, a concept for decoupling electrical and optical properties of the solar cell rear incorporating a photonic rear side structure was successfully demonstrated. 


\section{Open questions and outlook}

These results for the two exemplary applications of NIL for solar cell texturisation as well as the developed Roller-NIL tool reveal the high potential of this technology. Beyond the already ongoing or concrete next steps for both applications investigated within this work, which were already described in chapter 5, in the following some challenges for the industrial feasibility of this technology shall be described. There, the focus will be placed on the honeycomb texturing as this concept is closer to an industrial application. In general, these requirements also hold for the realisation of diffractive rear side gratings.

One important point about the up-scaleability of NIL is the stamp lifetime. Within this thesis, this issue was just investigated as a side topic. It was found that the stamp lifetime can vary tremendously using different resist materials. Diffusion of pre-polymers into the stamp is supposed to be one major problem altering the adhesion properties of the PDMS stamps to the resist. Thus, besides the chain length (or the molar weight) of the pre-polymers of the resist, the density of the porous network of the PDMS as well as surface energies of these materials will be import factors influencing the stamp's lifetime. Therefore, the scope of following works has to be the evaluation of different resist, PDMS and also other stamp materials and in particular the interaction of them.

The presented Roller-NIL tool already fulfils the requirements in terms of processable areas for the industrially applied wafer sizes. What has not been optimised up to now is the achievable processing velocity. The main factors influencing this process parameter are the necessary dose to cure the resist material and the filling time of the stamp's cavities. The first factor is related to both the kinetics for the curing of the resist and the power of the exposure source; the latter is related to the viscosity of the resist, surface energies of resist and stamp material as well as the dimensions of the stamp's features. Looking at the honeycomb texturing, velocities of the Roller-NIL process are not yet sufficient, but seem to be within reach.

The removal of resist residues after the etching processes was found to be a major topic within this work. Optimised plasma ashing processes were assessed as the best choice to guarantee a satisfactory removal. The rating of the different dry as well as wet-chemical removal techniques was performed with a relatively isolated view on this process step. Implementing the texturing scheme based on NIL into a full solar cell fabrication line, the choice of techniques will be more affected by the integral view of suitable processing sequences. It might turn out that a combination of wet-chemical resist removal with wet-chemical silicon etching could also be the most suitable solution. This was not evaluated in depth up to now. 
When sticking to the plasma etching as the technology for the pattern transfer into silicon, there are also some issues left to be solved. First of all, the large scale processing using sulphur hexafluoride as an etching gas, as was applied within this thesis, will not be reasonable because of its tremendous global warming potential. Therefore, plasma etching processes and tools being based on the use of molecular fluorine will have to be developed. To set up a complete dry texturing scheme (including the resist removal), the wet-chemical cleaning steps in between plasma processes that were applied in this work must be avoided.

In general, the implementation of plasma etching processes into solar cell texturisation might still be regarded as elaborate, although achievable optical texture quality is superior to wet-chemical approaches. If using vacuum technology, it would be best to execute as many as possible process steps based on dry techniques without locking out in between or maybe even to realise a complete dry processing scheme. One ideal front end processing sequence for the honeycomb texturing in a PERC cell might look like the following: starting with as-cut material, patterning of an etching mask via NIL, dry chemical etching and resist removal (enough material would have to be removed to exclude surface damages from the sawing process), rear side damage etching (ideally leading to a polished surface), PECVD deposition of front side passivation / ARC including dopants, PECVD deposition of rear side passivation and a firing process for the emitter formation. A process sequence like this might be advantageous compared to the established ones in terms of all steps being possible in single side treatment, suitability for very thin wafers, usage of ultra clean water and waste abatement.

The cost-benefit ratio of NIL processes, which is very important for an introduction into industrial environments, is difficult at the moment as e.g. the resist materials are not finally chosen yet and this will make up a considerable part of the running expenses. Furthermore, more data on solar cell efficiency enhancements, especially also for multicrystalline silicon, will be necessary to find out if this texturing scheme can be designed in a cost effective way. Within this work, the weighted reflectance of uncoated silicon samples was chosen as the figure of merit for the optical comparison of different front side textures to reduce the complexity. This allows a very sensitive assessment of optical texture quality, since when applying an ARC the differences in reflectivity will be reduced. Furthermore, the comparison of different textures is more convenient since for each specific texture an adaption of the ARC thickness would have to be performed. However, for a more detailed analysis, it will be important to compare the incorporation of different types of textures with ARC encapsulated into final modules. There, the benefit of the reduction of front surface reflectivity for honeycomb textures compared to the isotexture will shrink as a result of the 
gradually increased refractive index coming from air, through the module glass (and the encapsulant material EVA ${ }^{16}$ ) and the typically applied silicon nitride into the silicon. Thus, the optical gain might more a result of the superior light trapping effects. The same arguments also hold for solar cell efficiencies, which correctly have to be studied in the final stage of being encapsulated into a module. This will show if the defined texturing scheme can be performed cost effectively.

\section{Prospects of NIL for PV applications}

If these challenges can be met, many interesting developments are to be expected. Honeycomb texturing is of current interest for multicrystalline silicon, but will also be interesting for Quasi-Mono substrates or alternative wafering technologies of the future. The possibility of realising photonic structures in the sub-micron regime will be extremely interesting for very thin substrates. There are already some concepts relying e.g. on epitaxial growth and lift-off techniques for the realisation of such ultra-thin wafers with thicknesses in the range of several micrometers. The absence of saw damages and thus seeds for conventional texturing approaches as well as the necessity of removing only very little material will require novel texturing schemes as the one developed within this work.

Finally, also beyond texturisation, NIL will open up new possibilities for PV applications, wherever miniaturisation of device features is a topic. Therefore, this technology might be used as platform different stages in the solar cell process chain. This might be the high-resolution patterning of contact grids or diffusion barriers to optimise the device characteristics. Other applications related to optical effects might be the defined patterning of metal nanoparticles to make use of plasmonic effects or even the origination of complex photonic crystals.

\footnotetext{
${ }^{16}$ EVA: Ethylene vinyl acetate is a polymer material that is used as encapsulant for solar cells in modules. An important parameter of this material is that ist refractive index is very close to the cover glass and thus reflections at this interface can be minimised.
} 


\section{Deutsche Zusammenfassung}

Die Technologie der Nanoimprint Lithographie (NIL) eröffnet in punkto erreichbarer Strukturauflösungen bisher nicht denkbare Möglichkeiten für photovoltaische Anwendungen. Bisherige Entwicklungen zielten vorwiegend auf Anwendungen beispielsweise im Bereich der Mikroelektronik oder der Biowissenschaften ab. Aus diesem Grund lag die Zielstellung der vorliegenden Arbeit insbesondere auf der für die PV maßgeschneiderten Anlagen- und Prozessentwicklung dieser Technologie mit der Anwendung der Texturierung wafer-basierter Siliciumsolarzellen. Die NIL ist dabei lediglich ein Schritt in einer komplexen Prozesskette bestehend aus vorgeschalteten (Herstellung der Urform sowie Replikation polymerer Stempel) sowie sich wiederholender Schritte (NIL, Ätzen und Reinigung). Exemplarisch wurden zwei Anwendungsbeispiele für Oberflächentexturen untersucht, um das hohe Potential dieser innovativen Prozesskette zur Solarzellentexturierung aufzuzeigen. Dabei handelt es sich zum Einen um die Honeycomb-Texturierung, die insbesondere für multikristallines Silicium sehr interessant ist, sowie zum Anderen um die Realisierung diffraktiver Rückseitengitter als Photonenmanagement Strukturen, die speziell für zukünftige Zellgenerationen mit sehr dünnen Substraten wichtig werden können.

Die Herstellung von Urformen (oder auch Masterstrukturen genannt) wurde mittels Interferenzlithographie realisiert. Diese Technologie eignet sich insbesondere zur großflächigen Strukturierung periodischer Oberflächengitter mit Strukturgrößen im Nano- und Mikrobereich, wie sie im Rahmen dieser Arbeit zum Einsatz kamen. Von diesen Urformen wurden mittels Abgussverfahren Stempel in elastomeren Polymerwerkstoffen repliziert. Je nach Strukturgröße wurden hierzu unterschiedliche Stempelmaterialien untersucht. Stempel mit hochaufgelösten Strukturen (Liniengitter einer Periode wie auch einer Tiefe von etwa $300 \mathrm{~nm}$ ) konnten aus Schichtverbundwerkstoffen verschiedener Elastizitätsmoduln hergestellt werden.

Mit diesen UV-transparenten, flexiblen Stempeln sollte ein hochskalierbarer NIL Prozess entwickelt werden, um Ätzmasken aus UV-härtenden Resistmaterialien zu strukturieren. Zunächst wurde ein konventionelles Werkzeug zur UV-NIL auf Basis eines planaren Stempels konzipiert und hergestellt. Hierbei wurden Prägekräfte mittels Druckluft realisiert, um homogene Prägekräfte auf Flächen bis zu $100 \times 100 \mathrm{~mm}^{2}$ zu ermöglichen. Mit diesem Tool erfolgte eine Prozessentwicklung mit unterschiedlichen Lacksystemen. Die Resultate daraus flossen in die Konzeption eines Rollen-NIL Aufbaus ein. Hierzu wurde insbesondere das 
unterschiedliche Verhalten Lösemittel-basierter Epoxidlacke (SU8 von MicroChem) und Lösemittel-freier akrylischer Lacke (z.B. Laromer von BASF oder mr-UVCur06 von Micro Resist Technology) untersucht. Als Ergebnis zeigte sich, dass letztere sich prinzipiell besser für den sequentiellen Imprintprozess bei der Rollen-NIL eignen. Dies hängt mit den sich zeitlich verändernden rheologischen Eigenschaften Lösemittel-basierter Lacke aufgrund von Ausgasungen zusammen. Mit Hilfe des konventionellen NIL Werkzeugs wurden des Weiteren Plasmaätzprozesse für den Strukturübertrag sowie die Integration dieses Texturierungskonzepts in eine Prozesskette zur Herstellung von Solarzellen untersucht. Hierbei konnten bereits wichtige Erkenntnisse über kritische Aspekte bei dieser Integration gesammelt werden.

Auf Basis dieser Machbarkeitsstudie sowie den Voruntersuchungen wurde anschließend eine neuartige Rollen-NIL Anlage konzipiert und aufgebaut. Es gibt prinzipiell bestehende Anlagen, die bereits das hohe Potential von Imprintprozessen bezüglich Durchsatz sowie Strukturauflösungen zeigen. Hierbei handelt es sich jedoch typischerweise um Rollenprägeprozesse auf flexiblen Plastiksubstraten, die über ein System von Rollen geführt und prozessiert werden. Aus diesem Grund wird hier der bekannte Ausdruck von Rolle-zu-Rolle Prozessen verwendet. Anders als bei diesen Anlagen, müssen, bei der für die hier verfolgte Anwendung zu Entwickelnden, nicht transparente, steife, spröde und potentiell sehr raue Siliciumsubstrate prozessiert werden können. Diese Randbedingungen mussten bei der Anlagenentwicklung berücksichtigt werden und führten zu einem Aufbau, bei dem ein flexibler Stempel um einen Quarzglas Zylinder gespannt ist, in dem eine Belichtungseinheit aus UV-LEDs realisiert ist. Das mit Resist beschichtete Substrat wird auf einem Vakuumcarrier mittels eines Förderbandes unter der Rolle hindurchgeführt, wo der Lack im Kontakt mit dem Stempel ausgehärtet wird. Mit dieser innovativen Anlage konnten Ätzmasken für die Honeycombtextuierung (Periode wie auch Strukturtiefe ca. $8 \mu \mathrm{m}$ ) auf Flächen bis zu $156 \times 156 \mathrm{~mm}^{2}$ homogen und mit Restlackdicken unter $100 \mathrm{~nm}$ strukturiert werden. Die Anpassungsfähigkeit des Stempels an raue Oberflächen war dabei der des konventionellen Aufbaus zur NIL weit überlegen, was sich durch die geringere Kontaktfläche beim Rollenprägeprozess erklären lässt. Des Weiteren konnten sehr dünne, nur $100 \mu \mathrm{m}$ dicke multikristalline Substrate $\left(125 \times 125 \mathrm{~mm}^{2}\right)$ prozessiert werden, ohne dass Probleme eines möglichen Waferbruchs auftraten.

Die mittels NIL strukturierten Lackschichten dienten anschließend als Ätzmaske in Plasmaätzprozessen. Die Wahl für Plasma-basierte Ätzprozesse war durch die höchstmögliche Freiheit an realisierbaren Strukturformen und möglichst geringem Einfluss von Kristallorientierungen im Silicium begründet. Nach der Entwicklung 
von Ätz- sowie Reinigungsprozessen wurden Precursor sowie fertige Solarzellen basierend auf der entwickelten Prozesskette realisiert.

Im Kontext der Honeycombtexturierung konnten exzellente optische Eigenschaften der gefertigten Strukturen von einer gewichteten Reflektivität von $14 \%$ (ohne ARC) auf multikristallinen Substraten demonstriert werden. Dies konnte in einer Solarzellencharge auf FZ Material und kleinen Flächen $\left(2 \times 2 \mathrm{~cm}^{2}\right)$ über sehr hohe Kurzschlussstromdichten von $40.1 \mathrm{~mA} / \mathrm{cm}^{2}$ und $40.7 \mathrm{~mA} / \mathrm{cm}^{2}$ mit ein- und zweilagiger ARC bestätigt werden. Eine Quanteneffizienzanalyse verbunden mit einer Zellsimulation mit der Software PC1D bestätigte des Weiteren, dass diese neuartige Texturierungsprozesskette keine signifikante Erhöhung der Oberflächenrekombination bewirkt. Das Hochskalieren der Gesamt-Texturierungsprozesskette

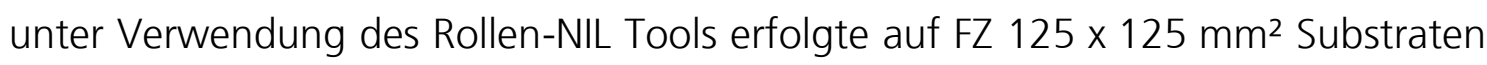
(durch die Plasmaanlage auf diese Größe limitiert). Hierbei konnten leider die zuvor ebenso großflächig demonstrierten optischen exzellenten Werte aufgrund von Prozessinstabilitäten beim Ätzen nicht reproduziert werden. Die Homogenität der Texturen auf großen Flächen war jedoch hervorragend. Aufgrund der moderaten Qualität der Textur konnten hier Kurzschlussstromdichten von ca. $35 \mathrm{~mA} / \mathrm{cm}^{2}$ erreicht werden. Bezüglich des Füllfaktors konnten im Vergleich zu ersten Chargen Fortschritte durch die Verwendung von Aerosol-Druck und Galvanik für die Vorderseitenmetallisierung erzielt werden (Füllfaktor von bis zu $77.7 \%$ ). Des Weiteren zeigte sich die Rekombination an der Vorderseite erneut nicht als limitierend (maximales $V_{o c}$ von $651 \mathrm{mV}$ für eine Aluminium BSF Zelle).

Als zweite Anwendung wurde die Realisierung diffraktiver Rückseitengitter untersucht. Hier wurden Linien- und Kreuzgitter einer Periode von $1 \mu \mathrm{m}$ realisiert. Dabei wurden Zell-Precursor hergestellt, die eine entspiegelte plane Vorderseite sowie eine dielektrische Pufferschicht und einen aufgedampften Aluminiumspiegel auf der strukturierten Rückseite aufwiesen. Bezüglich der optischen Absorptionserhöhung konnte in Übereinstimmung zur Literatur [95] gezeigt werden, dass Kreuzgitter ein höheres Potential haben. Diese führten zu Absorptionserhöhungen von bis zu $52 \%$ absolut bei einer Wellenlänge von etwa $1100 \mathrm{~nm}$ und einer Waferdicke von $200 \mu \mathrm{m}$. Die mittels PECVD abgeschiedenen Siliciumoxid-Pufferschichten zwischen Gitter und Rückseitenmetallisierung wuchsen konformal auf dem Gitter auf, sodass eine modulierte Oberfläche des metallischen Spiegels erzielt wurde. Dies führte zu beträchtlichen parasitären Absorptionen in der Metallschicht. Eine Optimierung hinsichtlich der optischen Güte des Rückseitenreflektors erfolgte mit der Zielsetzung diesen möglichst planar auszugestalten. Hierzu wurde ein Spincoating Verfahren zum Aufbringen einer wässrigen Dispersion von Siliciumoxidnanopartikeln erfolgreich untersucht. Der Vergleich der optischen Charakterisierungen der Proben mit wellenoptischen 
Simulationen, die von Alex Mellor an der Universität Madrid durchgeführt wurden, wies exzellente Übereinstimmungen auf. Parallel zur optischen Charakterisierung, wurde eine Zellstruktur untersucht, bei der optische und elektrische Eigenschaften der Rückseitenstrukturen entkoppelt werden können. Hierzu wurde die planare Rückseite mittels Aluminiumoxid passiviert. Auf dieser passivierten Oberfläche wurde anschließend amorphes Silicium abschieden, welches texturiert wurde. Bezüglich dieses Konzepts wurden Zell-Precursor realisiert, bei denen Lebensdauermessungen vor und nach dem Texturierungsprozess durchgeführt wurden. Auf diese Weise konnte gezeigt werden, dass die Entkopplung erfolgreich funktioniert und es zu keiner Beeinträchtigung der Oberflächenrekombination kommt. Abschließend wurde für die Rückseitengitter auf Basis der wellenoptischen Simulationen sowie der gemessenen Lebensdauern eine Effizienzabschätzung mittels PC1D durchgeführt. Hier wurde vorhergesagt, dass die Kreuzgitter, mit noch nicht optimaler Ausgestaltung des Rückseitenreflektors (PECVD $\mathrm{SiO}_{2}$ ), zu einem Effizienzgewinn von $0.9 \%$ absolut beziehungsweise $1.6 \%$ absolut für $200 \mu \mathrm{m}$ beziehungsweise $40 \mu \mathrm{m}$ dicke Zellen im Vergleich zu planen Referenzen führen. Dies sollte noch deutlicher ausfallen für die optimierten Rückreflektoren auf Basis des Spincoatings der Siliciumoxidschicht.

Die Ergebnisse der beiden exemplarischen Anwendungen der NIL für die Solarzellentexturierung sowie die neuartige Anlage zur Rollen-NIL zeigen das hohe Potential dieser Technologie für die PV auf. Über die Texturisierung hinaus kann die NIL als Technologieplattform für eine Vielzahl an Anwendungen im Bereich der PV interessant werden. Generell ist sie für jegliche Aspekte spannend bei der eine Miniaturisierung von Bauteildetails verfolgt wird. Dies könnten die Strukturierung von Diffusionsbarrieren für komplexe Solarzellenarchitekturen oder die Realisierung feinster Leiterbahnen sein. Spannende optische Anwendungen, die überhaupt erst durch die Möglichkeiten dieser Technologie eröffnet werden könnten, basieren beispielsweise auf der definierten Strukturierung von Metallnanopartikeln für plasmonische Anwendungen oder die Herstellung photonischer Kristalle. 


\section{References}

[1] Hering, G., Enter the dragon, Photon International, p. 142, March (2012).

[2] International Technology Roadmap for Photovoltaic (ITRPV.net) (2012).

[3] Ernst, M., Brendel, R., Ferré, R. and Harder, N.-P., Thin macroporous silicon heterojunction solar cells, physica status solidi (RRL) - Rapid Research Letters, 6 (5), 187-189, (2012).

[4] Henley, F., Lamm, A., Kang, S., Liu, Z. and Tian, L., Direct film transfer (DFT) technology for kerf-free silicon wafering, Proceedings of the 23rd European Photovoltaic Conference, (2008).

[5] Schultz, O., Glunz, S.W. and Willeke, G., Multicrystalline Silicon Solar Cells Exceeding $20 \%$ Efficiency, Progress in Photovoltaics: Research and Applications, 12 (7), 553-558, (2004).

[6] International Roadmap for Semiconductors, Lithography, (2003).

[7] Fairley, P., 10 Emerging Technologies That Will Change The World, Technology Review (2003).

[8] Sze, S.M., Semiconductor devices, Physics and Technology, 2nd ed. John Wiley \& Sons Inc. ,New York, USA (2002).

[9] Paul, A., Luisier, M., Neophytou, N., Kim, R., Geng, J., McLennan, M., Lundstrom, M. and Klimeck, G., Band Structure Lab, DOI: 10254/nanohubr1308.18(2011).

[10] Green, M.A., High efficiency silicon solar cells, Materials Science Surveys, ed. 5, Trans Tech Publications, Kensington (1987).

[11] Green, M.A., Silicon solar cells: advanced principles and practice, Centre for Photovoltaic Devises and Systems UNSW, Sydney, Australia (1995).

[12] Kray, D., Hocheffiziente Solarzellenstrukturen für kristallines SiliciumMaterial industrieller Qualität, Dissertation, Fakultät für Physik, Universität Konstanz, (2004).

[13] Clugston, D.A. and Basore, P.A., PC1D version 5: 32-bit solar cell modeling on personal computers, Proceedings of the 26th IEEE Photovoltaic Specialists Conference, IEEE; New York, NY, USA, 207-210 (1997).

[14] Sproul, A.B. and Green, M.A., Improved value for the silicon intrinsic carrier concentration from 275 to 375 K, Journal of Applied Physics, 70 (2), 846854, (1991).

[15] Glunz, S.W., Ladungsträgerrekombination in Silicium und Siliciumsolarzellen, Dissertation, Fakultät für Physik, Universität Freiburg, (1995).

[16] Shockley, W. and Read, W.T.J., Statistics of the recombinations of holes and electrons, Physical Review, 87 (5), 835-842, (1952). 
[17] Hall, R.N., Electron-hole recombination in germanium, Physical Review, 87 387, (1952).

[18] Schubert, M., Detektion von infraroter Strahlung zur Beurteilung der Materialqualität von Solar-Silizium, Dissertation, Fakultät für Physik, Universität Konstanz, (2008).

[19] Eades, W.D. and Swanson, R.M., Calculation of surface generation and recombination velocities at the $\mathrm{Si}_{-} \mathrm{SiO}_{2}$ interface, Journal of Applied Physics, 58 (11), 4267-4276, (1985).

[20] Aberle, A.G., Surface passivation of crystalline silicon solar cells: a review, Progress in Photovoltaics: Research and Applications, 2000 (8), 473-487, (2000).

[21] Schultz, O., High-efficiency multicrystalline silicon solar cells, Dissertation, Fakultät für Physik, Universität Konstanz, (2005).

[22] Goetzberger, A., Voß, B. and Knobloch, J., Sonnenenergie: Photovoltaik, Teubner Studienbücher Physik,Stuttgart (1994).

[23] Shockley, W. and Queisser, H.J., Detailed balance limit of efficiency of $p-n$ junction solar cells, Journal of Applied Physics, 32 (3), 510-519, (1961).

[24] Würfel, P. and Trupke, T., Solarzellen der dritten Generation, Physik Journal, 2 (12), 45-51, (2003).

[25] Kerr, M.J., Campbell, P. and Cuevas, A., Lifetime and efficiency limits of crystalline silicon solar cells, Proceedings of the 29th IEEE Photovoltaics Specialists Conference, 438-441 (2002).

[26] Benick, J., High-efficiency silicon solar cells with a front side boron emitter, University of Freiburg, (2010).

[27] Swanson, R.M., Beckwith, S.K., Crane, R.A., Eades, W.D., Kwark, Y.O., Sinton, R.A. and Swirhun, S.E., Point-contact silicon solar cells, IEEE Transactions on Electron Devices, 31 (5), 661 - 664, (1984).

[28] Glunz, S.W., High-efficiency crystalline silicon solar cells, Advances in OptoElectronics, 2007 97370, (2007).

[29] Würfel, P., Physik der Solarzellen, 2, Spektrum Akademischer Verlag Heidelberg, Berlin (2000).

[30] Sakata, H., Tsunomura, Y., Inoue, H., Taira, S., Baba, T., Kanno, H., Kinoshita, T., Taguchi, M. and Maruyama, E., R\&D progress of nextgeneration very thin HIT solar cells, Proceedings of the 25th European Photovoltaic Solar Energy Conference, (2010).

[31] Zhao, J., Wang, A. and Green, M.A., 24.5\% Efficiency silicon PERT cells on MCZ substrates and $24.7 \%$ efficiency PERL cells on FZ substrates, Progress in Photovoltaics: Research and Applications, 7 (6), 471-474, (1999).

[32] Green, M., Emery, K., Hishikawa, Y. and Warta, W., Solar cell efficiency tables (version 35), Progress in Photovoltaics: Research and Applications, 18 144-150, (2010). 
[33] IEC, Photovoltaic devices - part 3: measurement principles for terrestrial photovoltaic (PV) solar devices with reference spectral irradiance data. , 2nd ed. International Standard, IEC 60904-3, ed. International Electrotechnical Commission (2008).

[34] Peterson, K.E., Silicon as a mechanical material, Proceedings of the IEEE, 70 (5), 420 - 457, (1982).

[35] Sarti, D. and Einhaus, R., Silicon feedstock for the multi-crystalline photovoltaic industry, Solar Energy Materials and Solar Cells, 72 27-40, (2002).

[36] Q-Cells, Product datasheet multicrystalline solar cells, Q6LTT3, (2011).

[37] Wolf, F.V., EFG crystal growth technology for low cost terrestrial photovoltaics: review and outlook, Solar Energy Materials \& Solar Cells, 23 (2 - 4), 175 - 182, (1991).

[38] Lee, D.B., Anisotropic etching of silicon, Journal of Applied Physics, 40 (11), 4569-4574, (1969).

[39] Campbell, P. and Green, M.A., Light trapping properties of pyramidally textured surfaces, Journal of Applied Physics, 62 (1), 243-249, (1987).

[40] Hauser, A., Melnyk, I., Fath, P., Narayanan, S., Roberts, S. and Bruton, T.M., A simplified process for isotropic texturing of mc-Si, Proceedings of $3 \mathrm{rd}$ World Conference on Photovoltaic Energy Conversion, 2, 1447-1450 (2003).

[41] Beaucarne, G., Choulat, P., Chan, B.T., Dekkers, H., John, J. and Poortmans, J., Etching, texturing and surface decoupling for the next generation of Si solar cells, Photovoltaics International, 1st edition (2008).

[42] Gindner, S., Junge, J., Seren, S. and Hahn, G., Texturing of string ribbon silicon, Proceedings of the 23rd European Photovoltaic Conference, (2010).

[43] Green, M.A., Solar cells: operating principles, technology and system applications, The University of New South Wales, Kensington (1998).

[44] Mette, A., New concepts for front side metallization of industrial silicon solar cells, Dissertation, Fakultät für Angewandte Wissenschaften, Universität Freiburg, (2007).

[45] Kray, D., Hermle, M. and Glunz, S.W., Theory and experiment on the back side reflectance of silicon wafer solar cells, Progress in Photovoltaics: Research and Applications, 16 1-15, (2008).

[46] Basore, P.A., Numerical modeling of textured silicon solar cells using PC-1D, Proceedings of the 21st IEEE Photovoltaic Specialists Conference, 37, 337343 (1990).

[47] Basore, P.A., Extended spectral analysis of internal quantum efficiency, Proceedings of the 23rd IEEE Photovoltaic Specialists Conference, IEEE; New York, NY, USA, 147-152 (1993). 
[48] Baker-Finch, S.C. and Mclntosh, K.R., A freeware model for precise optical analysis of the front surface of a solar cell, Proceedings of the 35th IEEE photovoltaics specialists conference, 2184 - 2187 (2010).

[49] Schneiderlöchner, E., Preu, R., Lüdemann, R. and Glunz, S.W., Laser-fired rear contacts for crystalline silicon solar cells, Progress in Photovoltaics: Research and Applications, 10 29-34, (2002).

[50] Heine, C. and Morf, R.H., Submicrometer gratings for solar energy applications Applied Optics, 34 (14), 2476-2482, (1995).

[51] Hofmann, M., Rear surface conditioning and passivation for locally contacted crystalline silicon solar cells, Dissertation, Fachbereich Physik, Universität Konstanz, (2008).

[52] Dale, B. and Rudenberg, H.G., Photovoltaic conversion, 1. High efficiency silicon solar cells, 14th annual power sources conference, 22-25 (1960).

[53] Harbecke, B., Coherent and incoherent reflection and transmission of multilayer structures, Applied Physics B, 39 165-170, (1986).

[54] Peters, M., Bielawny, A., Bläsi, B., Carius, R., Glunz, S.W., Goldschmidt, J.C., Hauser, H., Hermle, M., Kirchartz, T., Löper, P., Üpping, J., Wehrspohn, R. and Willeke, G., Photonic Concepts for Solar Cells, in Physics of Nanostructured Solar Cells, V. Badescu, Editor, Nova Science Publishers (2010).

[55] Gombert, A., Large-area micro-structured surfaces with optical functions, Habilitation, Universität Freiburg, (2004).

[56] Bernhard, D.G., Structural and functional adaption in a visual system, Endeavour, 2679 - 84, (1967).

[57] Clapham, P.B. and Hutley, M.C., Reduction of lens reflection by the , moth eye' principle, Nature, 244281 - 282, (1973).

[58] Bläsi, B., Holographisch hergestellte Antireflexoberflächen für solare und visuelle Anwendungen, Dissertation, University of Freiburg, (2000).

[59] Goetzberger, A., Optical confinement in thin Si-solar cells by diffuse back reflectors, Proceedings of the 15th IEEE Photovoltaic Specialists Conference, 867-870 (1981).

[60] Yablonovitch, E., Statistical ray optics, Journal of the Optical Society of America, 72 (7), 899-907, (1982).

[61] Sheng, P., Optical absorption of thin film on a lambertian reflector substrate, IEEE Transactions on Electron Devices, 31 (5), 634-636, (1984).

[62] Minano, J.C., Optical confinement in photovoltaics, in Physical limitations to photovoltaic energy conversion, A. Luque and G.L. Araujo, Editors, Institute of Physics Publishing. p. 50-83 (1990).

[63] Mellor, A., Tobias, I., Marti, A. and Luque, A., A numerical study of Biperiodic binary diffraction gratings for solar cell applications, Solar Energy Materials \& Solar Cells, 95 (12), 3527 - 3535 (2011). 
[64] Peters, M., Rüdiger, M., Hauser, H., Hermle, M. and Bläsi, B., Diffractive gratings for crystalline silicon solar cells-optimum parameters and loss mechanisms, Progress in Photovoltaics: Research and Applications, published online, (2011).

[65] St. John, A.E., Multiple internal reflection structure in a silicondetector which is obtained by sandblasting, Patent, US 3.487.223, (1969).

[66] Arndt, R.A., Allison, J.F., Haynos, J.G. and Meulenberg, A.J., Optical properties of the COMSAT non-reflective cell, Proceedings of the 11 th IEEE Photovoltaic Specialists Conference, 40-43 (1975).

[67] Robbins, H. and Schwartz, B., Chemical etching of silicon I. The system HF, $\mathrm{HNO}_{3}$, and $\mathrm{H}_{2} \mathrm{O}$, Journal of the Electrochemical Society, 106 (6), 505-508, (1959).

[68] de Wolf, S., Choulat, P., Vazsonyi, E., Einhaus, R., Van Kerschaver, E., De Clercq, K. and Szlufcik, J., Towards industrial application of isotropic texturing for multi-crystalline silicon solar cells, Proceedings of the 16th European Photovoltaic Solar Energy Conference, James \& James, London, UK, 2000, 1521-1523 (2000).

[69] Vazsonyi, E., de Clercq, K., Einhaus, R., Van Kerschaver, E., Said, K., Poortmans, J., Szlufcik, J. and Nijs, J., Improved anisotropic etching process for industrial texturing of silicon solar cells, Solar Energy Materials and Solar Cells, 57 (2), 179-188, (1999).

[70] Kulkarni, M.S. and Erk, H.F., Acid-based etching of silicon wafers: masstransfer and kinetic effects, Journal of the Electrochemical Society, 147 (1), 176-188, (2000).

[71] Macdonald, D.H., Cuevas, A., Kerr, M.J., Samundsett, C., Ruby, D., Winderbaum, S. and Leo, A., Texturing industrial multicrystalline silicon solar cells, Solar Energy, 76 277-283, (2004).

[72] Baker-Finch, S.C. and Mclntosh, K.R., Reflection of normally incident light from silicon solar cells with pyramidal texture, Progress in Photovoltaics: Research and Applications, 19 (4), 406-416, (2011).

[73] Bilyalov, R.R., Lüdemann, R., Wettling, W., Stalmans, L., Poortmans, J., Nijs, J., Schirone, L., Sotgiu, G., Strehlke, S. and Lévy-Clément, C., Multicrystalline silicon solar cells with porous silicon emitter, Solar Energy Materials and Solar Cells, 60 (4), 391 - 420, (2000).

[74] Bastide, S., Albu-Yaron, A., Strehlke, S. and Lévy-Clément, C., Formation and characterization of porous silicon layers for application in multicrystalline silicon solar cells, Solar Energy Materials \& Solar Cells, 57 (4), 393 - 417, (1999).

[75] Strehlke, S., Sarti, D., Krotkus, A., Grigoras, K. and Lévy-Clément, C., The porous silicon emitter concept applied to multicrystalline silicon solar cells, Thin Solid Films, 297 (1 -2), 291 - 295, (1997).

[76] Jansen, H., de Boer, M., Legtenberg, R. and Elwenspoek, M., The black silicon method: a universal method for determining the parameter setting 
of a fluorine-based reactive ion etcher in deep silicon trench etching with profile control, Journal of Micromechanics and Microengineering, 5115 120, (1995).

[77] Schnell, M., Lüdemann, R. and Schaefer, S., Plasma surface texturization for multicrystalline silicon solar cells, IEEE Electron Devices SocProceedings of the 28th IEEE Photovoltaics Specialists Conference, 367-370 (2000).

[78] Schaefer, S., Plasmaätzen für die Photovoltaik, Dissertation, Universität Konstanz, (2000).

[79] Gerhards, C., Marckmann, C., Tolle, R., Spiegel, M., Fath, P., Willeke, G., Bucher, E., Creager, J. and Narayanan, S., Mechanically V-textured low cost multicrystalline silicon solar cells with a novel printing metallization, Proceedings of the 26th IEEE Photovoltaic Specialists Conference, IEEE; New York, NY, USA, 43-46 (1997).

[80] Willeke, G., Nussbaumer, H., Bender, H. and Bucher, E., A simple and effective light trapping technique for poycrystalline silicon solar cells, Solar Energy Materials and Solar Cells, 26 (4), 345-356, (1992).

[81] Zolper, J.C., Narayanan, S., Wenham, S.R. and Green, M.A., 16.7 \% efficient, laser textured, buried contact polycrystalline silicon solar cell, Applied Physics Letters, 55 (22), 2363-2365, (1989).

[82] Rentsch, J., Bamberg, F., Schneiderlöchner, E. and Preu, R., Texturing of multicrystalline silicon by laser ablation, Proceedings of the 20th European Photovoltaic Solar Energy Conference, 1321-1324 (2005).

[83] Menz, W. and Mohr, J., Mikrosystemtechnik für Ingenieure, 2nd edition ed. VCH (1997).

[84] Niinobe, D., Morikawa, H., Hiza, S., Sato, T., Matsuno, S., Fujioka, H., Katsura, T., Okamoto, T., Hamamoto, S., Ishihara, T. and Arimoto, S., Large-size multi-crystalline silicon solar cells with honeycomb textured surface and point-contacted rear toward industrial production, Solar Energy Materials \& Solar Cells, 95 (1), 49 - 52, (2010).

[85] Niinobe, D., Nishimura, K., Matsuno, S., Fujioka, H., Katsura, T., Okamoto, T., Ishihara, T., Morikawa, H. and Arimoto, S., Honeycomb-structured mulitcrystalline silicon solar cells with 18,6\% efficiency via industrially applicable laser process, Proceedings of the 23rd European Photovoltaic Solar Energy Conference, (2008).

[86] Nievendick, J., Specht, J., Zimmer, M., Zahner, L., Glover, W., Stüwe, D. and Rentsch, J., Formation of a honeycomb texture for multicrystalline silicon solar cells using an inkjetted mask, Physica Status Solidi RRL, 6 (1), 7 - 9, (2012).

[87] Borojevic, N., Lennon, A. and Wenham, S., Inkjet texturing for multicrystalline silicon solar cells, Proceedings of the 24th European Photovoltaic Solar Energy Conference, 1975-1978 (2009).

[88] Stocks, M.J., Carr, A.J. and Blakers, A.W., Texturing of polycrystalline silicon, Solar Energy Materials and Solar Cells, 40 (1), 33-42, (1996). 
[89] Zhao, J., Wang, A., Campbell, P. and Green, M.A., A 19.8\% efficient honeycomb multicrystalline silicon solar cell with improved light trapping, IEEE Transactions on Electron Devices, 46 (10), 1978-1983, (1999).

[90] Schultz, O., Emanuel, G., Glunz, S.W. and Willeke, G.P., Texturing of multicrystalline silicon with acidic wet chemical etching and plasma etching, Proceedings of the 3rd World Conference on Photovoltaic Energy Conversion, WCPEC-3 Organizing Committee, December 2003 2, 13601363 (2003).

[91] Zielke, D., Sylla, D., Neubert, T., Brendel, R. and Schmidt, J., Direct Laser Texturing for High-Efficiency Silicon Solar Cells, Photovoltaics, IEEE Journal of, PP (99), 1-6, (2012).

[92] Li, D.Y., Texturization of multicrystalline silicon solar cells, Dissertation, Massachusetts Institute of Technology (2010).

[93] Gale, M.T., Curtis, B.J., Kiess, H. and Morf, R., Design and fabrication of submicron grating structures for light trapping in silicon solar cells, Proc. SPIE 1272, 60 - 66 (1990).

[94] Battaglia, C., Hsu, C.-M., Söderström, K., Escarré, J., Haug, F.-J., Charrière, M., Boccard, M., Despeisse, M., Alexander, D.T.L., Cantoni, M., Cui, Y. and Ballif, C., Light Trapping in Solar Cells: Can Periodic Beat Random?, ACS Nano, 6 (3), 2790 - 2797, (2012).

[95] Mellor, A., Tobias, I., Marti, A., Mendes, M. and Luque, A., Upper limits to absorption enhancement in thick solar cells using diffraction gratings, Progress in Photovoltaics: Research and Applications, 19 (6), 676 - 687, (2011).

[96] Gjessing, J., Sudbø, A.S. and Marstein, E.S., Comparison of periodic lighttrapping structures in thin crystalline silicon solar cells, Journal of Applied Physics, 110 (3), 033104, (2011).

[97] Kiess, H., Morf, R., Gale, M.T. and Widmer, A.E., Light trappning in solar cells using submicron gratings, Technical digest of the international PVSEC, 5, (1990).

[98] Morf, R.H., Kiess, H. and Heine, C., Diffractive optics for solar cells, in Diffractive optics for industrial and commercial applications, J. Turunen and F. Wyrowski, Editors, Akademie Verlag: Berlin. p. 361-369 (1997).

[99] Wellenzohn, M. and Hainberger, R., Light trapping by backside diffraction gratings in silicon solar cells revisited, Optics Express, 20 (S1), (2012).

[100] Berger, P., Hauser, H., Suwito, D., Janz, S., Peters, M., Bläsi, B. and Hermle, M., Realization and evaluation of diffractive systems on the back side of silicon solar cells, Proc. SPIE 7725, (2010).

[101] Jin, H., Weber, K.J. and Blakers, A.W., Depassivation of Si-SiO2 interface following rapid thermal annealing, Proceedings of the 4th World Conference on Photovoltaic Energy Conversion, 1078-1080 (2006).

[102] Glunz, S.W., Sterk, S., Steeman, R., Warta, W., Knobloch, J. and Wettling, W., Emitter dark saturation currents of high-efficiency solar cells with 
inverted pyramids, Proceedings of the 13th European Photovoltaic Solar Energy Conference, H.S. Stephens \& Associates, Bedford, UK, 1995, 409412 (1995).

[103] Recard, F. and Cuevas, A., Application of junction capacitance measurements to the characterisation of solar cells, IEEE Transactions on Electron Devices, 53 (3), (2006).

[104] Cousins, P.J. and Cotter, J.E., Minimizing lifetime degradation associated with thermal oxidation of upright randomly textured silicon surfaces, Solar Energy Materials \& Solar Cells, 90 (2), 228-240, (2006).

[105] Mclntosh, K.R. and Johnson, L.P., Recombination at textured silicon surfaces passivated with silicon dioxide, Journal of Applied Physics, 105 124520, (2009).

[106] Baker-Finch, S.C. and McIntosh, K.R., The contribution of planes, vertices, and edges to recombination at pyramidally textured surfaces, IEEE Journal of Photovoltaics, 1 (1), 59-65, (2011).

[107] Terry, M.L., Meisel, A., Rosenfeld, E., Shah, S., Tai, E., Chen, X. and Du, T., All screen-printed $18 \%$ homogeneous emitter solar cells using high volume manufacturing equipment, Proceedings of the 35th IEEE Photovoltaic Specialists Conference (PVSC), (2010).

[108] Baker-Finch, S.C., Mclntosh, K.R., Terry, M.L. and Wan, Y., Isotextured Silicon Solar Cell Analysis and Modeling 2: Recombination and Device Modeling, IEEE Journal of Photovoltaics, 2 (4), (2012).

[109] Baker-Finch, S.C., Mclntosh, K.R., Inns, D. and Terry, M.L., Modelling Isotextured Silicon Solar Cells and Modules, Proceedings of the 38th IEEE Photovoltaic Specialists Conference (PVSC), (2012).

[110] Lüdemann, R., Damiani, B.M., Rohatgi, A. and Willeke, G., Silicon solar cells with black silicon texturization, Proceedings of the 17th European Photovoltaic Solar Energy Conference, WIP-Munich and ETA-Florence, 1327-1330 (2001).

[111] Zin, N., Blakers, A. and Weber, K., RIE-induced carrier lifetime degradation, Progress in Photovoltaics: Research and Applications, 18 (3), $214-220$ (2010).

[112] Otto, M., Kroll, M., Käsebier, T., Salzer, R., Tünnermann, A. and Wehrspohn, R.B., Extremely low surface recombination velocities in black silicon passivated by atomic layer deposition, Applied Physics Letters, 100 (19), 191603, (2012).

[113] Oh, J., Yuan, H.-C. and Branz, H.M., An 18.2\%-efficient black-silicon solar cell achieved through control of carrier recombination in nanostructures, Nature Nanotechnology (2012).

[114] Rentsch, J., Trockentechnologien zur Herstellung von kristallinen Siliziumsolarzellen, Dissertation, Albert-Ludwigs Universität, Freiburg, (2005). 
[115] Sparber, W., Schultz, O., Biro, D., Emanuel, G., Preu, R., Poddey, A. and Borchert, D., Comparison of texturing methods for monocrystalline silicon solar cells using $\mathrm{KOH}$ and $\mathrm{Na}_{2} \mathrm{CO}_{3}$, Proceedings of the 3rd World Conference on Photovoltaic Energy Conversion, WCPEC-3 Organizing Committee, December 2003 2, 1372-1375 (2003).

[116] Zechner, C., Mechanisch texturierte Solarzellen und RückkontaktSolarzellen aus kristallinem Silizium, Dissertation, Universität Konstanz, (1999).

[117] Bilyalov, R.R., Lautenschlager, H. and Schindler, R., Multicrystalline silicon solar cells with porous silicon selective emitter, Proceedings of the 2nd World Conference on Photovoltaic Energy Conversion, European Commission, Ispra, Italy, 1998, 1642-1645 (1998).

[118] Jessensky, O., Müller, F. and Gösele, U., Self-organized formation of hexagonal pore arrays in anodic alumina, Applied Physics Letters, 72 (10), 1173, (1998).

[119] Müller, M., Zentel, R., Maka, T., Romanov, S.G. and Sotomayor Torres, C.M., Dye-Containing Polymer Beads as Photonic Crystals, Chem. Mater., 12 (8), 2508 - 2512, (2000).

[120] Mihi, A., Ocaña, M. and Míguez, H., Oriented colloidal-crystal thin films by spin-coating microspheres dispersed in volatile media, Advanced Materials, 18 (17), 2244-2249, (2006).

[121] Zielke, D., Lim, B., Neubert, T., Sylla, D., Brendel, R. and Schmidt, J., Direct Laser Texturing of High-Efficiency Silicon Solar Cells, presented at the 27th European Photovoltaic Solar Energy Conference, (2012).

[122] Bietsch, A. and Michel, B., Conformal contact and pattern stability of stamps used for soft lithography, Journal of Applied Physics, 88 (7), 4310 4319, (2000).

[123] Plachetka, U., Bender, M., Fuchs, A., Vratzov, B., Glinsner, T., Lindner, F. and Kurz, H., Wafer scale patterning by soft UV-Nanoimprint Lithography, Microelectronic Engineering 73 - 74167 - 171, (2004).

[124] Gombert, A., Bläsi, B., Bühler, C., Nitz, P., Mick, J., Hoßfeld, W. and Niggemann, M., Some application cases and related manufacturing techniques for optically functional micro structures on large areas, Optical Engineering, 432525 - 2533 (2004).

[125] Gombert, A., Réseaux diffractifs d'ordre zéro comme surfaces antiréflection, application aux capteurs solaires, Dissertation, University of Strasbourg, (1998).

[126] Bläsi, B., Hauser, H., Höhn, O., Kübler, V., Peters, M. and Wolf, A.J., Photon management structures originated by interference lithography, Proceedings - 1st SiliconPV, April 17-20, ELSEVIER 8, 712-718 (2011).

[127] Hauser, H., Nanoimprint Lithographie mit interferenzlithographisch hergestellten Stempeln, Diploma Thesis, University of Freiburg, (2007). 
[128] Disch, A., Holographische Mikrostrukturierung prismatischer Oberflächen, Diploma Thesis, University of Freiburg, (2003).

[129] Dammel, R., Diazonaphtochinone-based resists, Tutorial texts in optical engineering, ed.,Published by SPIE - The International Society for Optical Engineering (1993).

[130] Mick, J., Interferenzlithographie mit hochaufbauenden Resistsystemen, Dissertation, University of Freiburg, (2005).

[131] Zanke, C., Optimierung des Herstellungsprozesses von großflächigen Subwellenlängengittern in Photoresist, Diploma Thesis, University of Applied Science Köln (1997).

[132] Chou, S.Y., Krauss, P.R. and Renstrom, P.J., Imprint of sub-25 $\mathrm{nm}$ vias and trenches in polymers, Applied Physics Letters, 67 (21), 3114 - 3116, (1995).

[133] Schift, H., Nanoimprint lithography: An old story in modern times? A review, Journal of Vacuum Science \& Technology B, 26 (2), (2008).

[134] Scheer, H.-C., Wissen, M., Bogdanksi, N. and Möllenbeck, S., Issues and Requirements of Polymers for Thermal NIL, Journal of Photopolymer Science and Technology, 20 (4), 539 - 544, (2007).

[135] Haisma, J., Verheijein, M., van den Heuvel, K. and van den Berg, j., Mold assisted nanolithography: a process for reliable pattern replication, Journal of Vacuum Science \& Technology B, 14 (6), 4124 - 4129, (1996).

[136] Guo, L.J., Nanoimprint Lithography: Methods and Material Requirements, Advanced Materials, 19 (4), 495 - 513, (2007).

[137] H. Schift, NaPa Library of Processes, NaPa-consortium represented by J. Ahopelto, 1st edition, (2008). 978-3-00-024396-7

[138] Tan, H., Kong, L., Li, M., Steer, C. and Koecher, L., Current Status of Nanonex Nanoimprint Solutions, Proceedings of the SPIE, Volume 5374, $213-221$ (2004).

[139] Microchem, NANOTM SU-8 2000 Negative Tone Photoresist Formulations $2002-2025$.

[140] BASF, LucirinTM TPO - Technical Information.

[141] Guo, L.J., Recent progress in nanoimprint technology and its applications, Journal of Physics D: Applied Physics, 37 (11), R123, (2004).

[142] Bailey, T.C., Colburn, M.E., Choi, B.J., Grot, A., Ekerdt, J.G., Sreenivasan, S.V. and Willson, C.G., Step and Flash Imprint Lithography: A Low-Pressure, Room Temperature Nanoimprint Patterning Process, in Alternative Lithography. Unleashing the Potentials of Nanotechnology, C.S. Torres, Editor, Elsevier (2002).

[143] Hauser, H., Michl, B., Schwarzkopf, S., Kübler, V., Müller, C., Hermle, M. and Bläsi, B., Honeycomb texturing of silicon via nanoimprint lithography for solar cell applications, IEEE Journal of Photovoltaics, 2 (2), 114-122, (2012). 
[144] Schift, H. and Kristensen, A., Nanoimprint Lithography - Patterning of resists using molding, in Springer Handbook of Nanotechnology, Part A. p. 271 - 312 (2010).

[145] Verschuuren, M., Substrate Conformal Imprint Lithography for Nanophotonics, Dissertation, University of Utrecht, (2010).

[146] Schmid, H. and Michel, B., Siloxane Polymers for High-Resolution, HighAccuracy Soft Lithography, Macromolecules, 33 (8), 3042 - 3049, (2000).

[147] Barbero, D.R., Saifullah, M.S.M., Hoffmann, P., Mathieu, H.J., Anderson, D., Jones, G.A.C., Welland, M.E. and Steiner, U., High Resolution Nanoimprinting with a Robust and Reusable Polymer Mold, Advanced Functional Materials, 17 (14), 2419 - 2425 (2007).

[148] Odom, T.W., Love, J.C., Wolfe, D.B., Paul, K.E. and Whitesides, G.M., Improved Pattern Transfer in Soft Lithography Using Composite Stamps, Langmuir, 18 (13), 5314 - 5320 (2002).

[149] Gates, B.D., Xu, Q., Stewart, M., Ryan, D., Willson, C.G. and Whitesides, G.M., New Approaches to Nanofabrication: Molding, Printing, and Other Techniques, Chem. Rev., 105 (4), 1171 - 1196, (2005).

[150] Schift, H., Saxer, S., Park, S., Padeste, C., Pieles, U. and Gobrecht, J., Controlled co-evaporation of silanes for nanoimprint stamps, Nanotechnology, 16 (5), 171 - 175, (2005).

[151] H. Tan, A.G.a.S.Y.C., Roller nanoimprint lithography, Journal of Vacuum Science \& Technology B, 16 (6), (1998).

[152] Vogler, M., Wiedenberg, S., Mühlberger, M., Bergmair, I., Glinsner, T., Schmidt, H., Kley, E.-B. and Grützner, G., Development of a novel, lowviscosity UV-curable polymer system for UV-nanoimprint lithography, Microelectronic Engineering, 84 (5 - 8), 984 - 988, (2007).

[153] Decker, C., Thi Viet, T.N., Decker, D. and Weber-Koehl, E., UV-radiation curing of acrylate/epoxide systems, Polymers, 42 (13), 5531 - 5541, (2001).

[154] Senn, T., Müller, C. and Reinecke, H., Replication of HARMST and large area nanostructured parts using UV cationic polymerization, Journal of Micromechanics and Microengineering, 20 (7), 075002, (2010).

[155] Lorenz, H., Despont, M., Fahrni, N., LaBianca, N., Renaud, P. and Vettiger, P., SU-8: a low-cost negative resist for MEMS, Journal of Micromechanics and Microengineering, 7 (3), 121 (1997).

[156] Chapman, B., Glow Discharge Processes - Sputtering and Plasma etching, New York: John Wiley \& Sons (1980).

[157] Dentinger, P.M., Clift, W.M. and Goods, S.H., Removal of SU-8 photoresist for thick film applications, Microelectronic Engineering, 61 - 62993 - 1000, (2002).

[158] Mathuni, J., Engelke, R., Löchel, B., Gutsche, M. and Genolet, G., Schnellstes Fertigungsverfahren für Epoxid-Removal, Werkstattstechnik online, Jahrgang 98 (11./12.), (2008). 
[159] Engelke, R., Mathuni, J., Ahrens, G., Gruetzner, G., Bednarzik, M., Schondelmaier, D. and Loechel, B., Investigations of SU-8 removal from metallic high aspect ratio microstructures with a novel plasma technique, Microsystem Technologies 14 (9 - 11), 1607 - 1612 (2008).

[160] Lieberman, M.A. and Lichtenberg, A.J., Principles of plasma discharges and materials processing, John Wiley \& Sons, Inc. (1994).

[161] Kashkoush, I.I., Matthews, R. and Novak, R.E., Photoresist Stripping Using Ozone/Deionized Water Chemistry, Proceedings of the Fifth International Symposium on Cleaning Technology in Semiconductor Device Manufacturing, 471 - 479 (1997).

[162] Microchem, Material Safety Data Sheet for SU-8 2000 Series Resists.

[163] Voicu, N.E., Ludwigs, S., Crossland, E.J.W., Andrew, P. and Steiner, U., Solvent-Vapor-Assisted Imprint Lithography, Advanced Materials, 19 (5), 757 - 761, (2007).

[164] Aldrich, S., Product information W391018 Cyclopentanone.

[165] Nielsen, T.D., Cruickshank, C., Foged, S., Thorsen, J. and Krebs, F.C., Business, market and intellectual property analysis of polymer solar cells, Solar Energy Materials \& Solar Cells, 94 (10), 1553 - 1571, (2010).

[166] Ahn, S.H. and Guo, L.J., High-Speed Roll-to-Roll Nanoimprint Lithography on Flexible Plastic Substrates, Advanced Materials, 20 (11), 2044 - 2049, (2008).

[167] Chang, C.Y., Yang, S.Y. and Sheh, J.L., A roller embossing process for rapid fabrication of microlens arrays on glass substrates, Microsystem Technologies, 12 (8), 754 - 759, (2006).

[168] Hubbard, G., Abbott, S.J., Chen, Q., Allsopp, D.W.E., Wang, W.N., Bowen, C.R., Stevens, R., Satka, A., Hasko, D., Uherek, F. and Kovac, J., Wafer-scale transfer of nanoimprinted patterns into silicon substrates, Physica E: Lowdimensional Systems and Nanostructures, 41 (6), 1118-1121, (2009).

[169] Schift, H., Roll Embossing and Roller Imprint, in Science and new technology in nanoimprint (2006).

[170] Ahn, S.H. and Guo, L.J., Large-area roll-to-roll and roll-to-plate nanoimprint lithography: A step toward high-throughput application of continuous nanoimprinting, ACS Nano, 3 (8), 2304 - 2310, (2009).

[171] Stenzel, V., Kaune, M. and Silva Branco Cheta, M.R., Werkzeug zur Erzeugung mikrostrukturierter Oberflächen, Patent, (2005).

[172] Schwarzkopf, S., Konzeption und Aufbau einer Rollen-UVNanoimprintanlage, Diploma Thesis, University of Karlsruhe (2010).

[173] Wang, X., Chen, Y., Banu, S., Morgan, H., Fu, S. and Cui, Z., High density patterns fabricated in SU-8 by UV curing nanoimprint, Microelectronic Engineering, 84 (5 - 8), 872 - 876, (2007).

[174] Laermer, F., Method of anisotropic etching of silicon, Patent, DE 4241045, (2003). 
[175] Jansen, H., Gardeniers, H., de Boer, M., Elwenspoek, M. and Fluitman, J., A survey on the reactive ion etching of silicon in microtechnology, Journal of Micromechanics and Microengineering, 6 (1), 14 - 28 (1996).

[176] Rentsch, J., Jaus, J., Roth, K. and Preu, R., Economical and ecological aspects of plasma processing for industrial solar cell fabrication Proceedings of the 31st IEEE Photovoltaic Specialists Conference, 931-934 (2005).

[177] de Wild-Scholten, M.J., Alsema, E.A., Fthenakis, V.M., Agostinelli, G., Dekkers, H. and Kinzig, V., Fluorinated greenhouse gases in photovoltaic module manufacturing: potential emissions and abatement strategies, Proceedings of the 22nd European Photovoltaic Solar Energy Conference, 1-14 (2007).

[178] Schottler, M. and de Wild-Schotten, M.J., Carbon footprint of PECVD chamber cleaning, Photovoltaics International 2nd edition 64 - 69 (2008).

[179] Lüdemann, R., Plasmatechnologie für die Photovoltaik, Dissertation, Fakultät für Physik, Universität Konstanz, (1999).

[180] Schaefer, S., Lüdemann, R., Lautenschlager, H., Juch, M. and Siniaguine, O., An overview of plasma sources suitable for dry etching of solar cells, Proceedings of the 28th IEEE Photovoltaics Specialists Conference, 79-82 (2000).

[181] Hofmann, M., Rentsch, J. and Preu, R., Dry plasma processing for industrial crystalline silicon solar cell production, The European Physical Journal Applied Physics, 5211101 (2010).

[182] Seiffe, J., Pillath, F., Trogus, D., Brand, A.A., Savio, C., Hofmann, M. and Rentsch, J., Multifunctional PECVD Layers: Dopant Source, Passivation, and Optics, IEEE Journal of Photovoltaics, (2012).

[183] Inomata, Y., Fukui, K. and Shirasawa, K., Surface texturing of large area multicrystalline silicon solar cells using reactive ion etching method, Solar Energy Materials \& Solar Cells, 48 (1 - 4), 237-242, (1997).

[184] Pfeiffer, M., Optimierung von Light-Trapping-Strukturen zur Effizienzsteigerung multikristalliner Siliziumsolarzellen, Diploma Thesis, University of Freiburg, (2008).

[185] Moharam, M.G. and Gaylord, T.K., Rigorous coupled-wave analysis of planar - grating diffraction, Journal of the Optical Society of America, 71 (7), $811-818,(1081)$.

[186] Zanke, C., Gombert, A., Erdmann, A. and Weiss, M., Fine-tuned profile simulation of holographically exposed photoresist gratings, Optics Communications, 154 (1-3), 109-118, (1998).

[187] Sinton, R.A., Cuevas, A. and Stuckings, M., Quasi-steady-state photoconductance, a new method for solar cell material and device characterization, Proceedings of the 25th IEEE Photovoltaic Specialists Conference, IEEE; New York, NY, USA, 457-460 (1996).

[188] Hauser, H., Voisin, P., Guttowski, A., Mick, J., Pfeifer, M., Müller, C., Hermle, M., Glunz, S. and Bläsi, B., Honeycomb textured multicrystalline 
silicon via nanoimprint lithography, Proceedings of the 24th European Photovoltaic Solar Energy Conference, 1118-1122 (2009).

[189] Ngo, H.-D., Hiess, A., Seidemann, V., Studzinski, D., Lange, M., Leib, J., Shariff, D., Ashraf, H., Steel, M., Atabo, L. and Reast, J., Plasma Etching of Tapered Features in Silicon for MEMS and Wafer Level Packaging Applications, Journal of Physics: Conference Series, 34271 - 276 (2006).

[190] Kim, S.W., Lee, H.J. and Seo, I.S., Method of removing polymer of semiconductor device, Patent, (1999).

[191] Walk, C., Honeycomb-Texturierung von Silicium mittels Rollen-UVNanoimprint Lithographie und Plasmaätzen, Diploma Thesis, University of Freiburg, (2012).

[192] Mathuni, J., UFER Investigations of SU-8 removal from metallic high aspect ratio microstructures with a novel plasma technique, Presentation at MiNaT HotSpots, (2008).

[193] Hauser, H., Michl, B., Kübler, V., Schwarzkopf, S., Müller, C., Hermle, M. and Bläsi, B., Nanoimprint lithography for honeycomb texturing of multicrystalline silicon, Energy Procedia, 8 648-653, (2011).

[194] Kane, D.E. and Swanson, R.M., Measurement of the emitter saturation current by a contactless photoconductivity decay method (silicon solar cells), Proceedings of the 18th IEEE Photovoltaic Specialists Conference, 578-583 (1985).

[195] Reichel, C., Granek, F., Benick, J., Schultz-Wittmann, O. and Glunz, S.W., Comparison of emitter saturation current densities determined by quasisteady-state photoconductance measurements of effective carrier lifetimes at high and low injections, Proceedings of the 23rd European Photovoltaic Solar Energy Conference, 1664-1669 (2008).

[196] Hauser, H., Michl, B., Schwarzkopf, S., Müller, C., Hermle, M. and Bläsi, B., Development of nanoimprint lithography for solar cell texturisation, Preceedings of the 25th European Photovoltaic Solar Energy Conference, 2171-2175 (2010).

[197] Kray, D. and Mclntosh, K.R., Analysis of selective phosphorous laser doping in high-efficiency solar cells, IEEE Transactions on Electron Devices, 56 (8), 1645-1650, (2009).

[198] Bläsi, B., Peters, M., Goldschmidt, J.C., Hermle, M., Hauser, H. and Voisin, P., Solarzelle und Verfahren zu deren Herstellung Patent, DE102009029944A1, (2010).

[199] Pang, S.W., Rathman, D.D., Silversmith, D.J., Mountain, R.W. and DeGraff, P.D., Damage induced in Si by ion milling or reactive ion etching, Journal of Applied Physics, 54 (6), 3272 - 3277, (1983).

[200] Zaidi, S.H., Ruby, D.S. and Gee, J.M., Characterization of Random Reactive Ion Etched-Textured Silicon Solar Cells, IEEE Transactions on Electron Devices, 48 (6), (2001). 
[201] Hermle, M., Bläsi, B., Peters, M., Goldschmidt, J.C., Hauser, H. and Voisin, P., Siliziumsolarzelle mit nanostrukturierter a-Si Rückseite, Patent, DE102009042018A1, (2011).

[202] Gjessing, J., Marstein, E.S. and Sudbø, A., 2D back-side diffraction grating for improved light trapping in thin silicon solar cells, Opt. Express, 18 (6), 5481-5495, (2010).

[203] Springer, J., Poruba, A., Mullerova, L., Vanecek, M., Kluth, O. and Rech, B., Absorption loss at nanorough silver back reflector of thin-film silicon solar cells, Journal of Applied Physics, 95 (3), 1427-1429, (2004).

[204] Guttowski, A., Enhanced Light Trapping for Silicon Thin Film Solar Cells, Master Thesis, University of Freiburg, (2011).

[205] Schaab, D., Surface patterning by means of Soft Lithography for Molecular and Bio-Electronics, Dissertaion, RWTH Aachen, (2007).

[206] Heidari, B., Beck, M. and Keil, M., Imprint stamp comprising Cyclic Olefin copolymer, Patent, US 20070212522A1, (2007).

[207] Weiss, D.N., Meyers, S.T. and Keszler, D.A., All-inorganic thermal nanoimprint process, Journal of Vacuum Science \& Technology B, 28 (4), 823 - 829, (2010).

[208] Topas Advanced Polymers GmbH, Datasheet: Topas® 5013L-10.

[209] Gilles, S., Meier, M., Prömpers, M., van der Hart, A., Kügeler, C., Offenhäusser, A. and Mayer, D., UV Nanoimprint Lithography with rigid stamps, Microelectronic Engineering, 86 (4 - 6), 661 - 664, (2009).

[210] Guttowski, A., Nanoimprint Lithographie auf rauen Substraten, Bachelor Thesis, University of Freiburg, (2008).

[211] Microchem, Datasheet Omnicoat.

[212] Micro-Resist-Technology, Datasheet mr-UVCur06.

[213] Kim, S.-K., Ee, H.-S., Choi, W., Kwon, S.-H., Kang, J.-H., Kim, Y.-H., Kwon, H. and Park, H.-G., Surface-plasmon-induced light absorption on a rough silver surface Applied Physics Letters, 98 (1), 011109, (2011).

[214] Söderström, K., Haug, F.J., Escarré, J., Pahud, C., Biron, R. and Ballif, C., Highly reflective nanotextured sputtered silver back reflector for flexible high-efficiency $n-i-p$ thin-film silicon solar cells, Solar Energy Materials and Solar Cells, 95 (12), 3585-3591, (2011).

[215] Mellor, A., Hauser, H., Guttowski, A., Wellens, C., Bläsi, B., Tobías, I., Martí, A. and Luque, A., Nano-imprinted rear-side diffraction gratings for absorption enhancement in solar cells, Proc. SPIE 8471, Next Generation (Nano) Photonic and Cell Technologies for Solar Energy Conversion III: 847100-847100, (2012).

[216] Mellor, A., Hauser, H., Wellens, C., Benick, J., Eisenlohr, J., Peters, M., Guttowski, A., Tobías, I., Martí, A., Luque, A. and Bläsi, B., Nanoimprinted diffraction gratings for crystalline silicon solar cells: implementation, 
characterisation and simulation, Optics Express, 21 (S2), A295-A304, (2013).

[217] Hauser, H., Mellor, A., Guttowski, A., Wellens, C., Benick, J., Müller, C., Hermle, M. and Bläsi, B., Diffractive backside structures via nanoimprint lithography, Energy Procedia, 27 (0), 337-342, (2012).

[218] Hoex, B., Heil, S.B.S., Langereis, E., van de Sanden, M.C.M. and Kessels, W.M.M., Ultralow surface recombination of c-Si substrates passivated by plasma-assisted atomic layer deposited $\mathrm{Al}_{2} \mathrm{O}_{3}$, Applied Physics Letters, 89 (042112), 042112/042111-042113, (2006).

[219] Fischer, B., Loss analysis of crystalline silicon solar cells using photoconductance and quantum efficiency measurements, Dissertation, Fachbereich Physik, Universität Konstanz, (2003).

[220] Rüdiger, M. and Hermle, M., Numerical Analysis of Locally Contacted Rear Surface Passivated Silicon Solar Cells, Japanese Journal of Applied Physics, 51 10NA07, (2012).

[221] Cuevas, A., Basore, P.A., Giroult-Matlakowski, G. and Dubois, C., Surface recombination velocity of highly doped n-type silicon, Journal of Applied Physics, 80 (6), 3370-3375, (1996).

[222] Macleod, H.A., Thin-film optical filters, Adam Hilger (1986).

[223] Kroll, M., Otto, M., Käsebier, T., Füchsel, K., Wehrspohn, R., Kley, E.-B., Tünnermann, A. and Pertsch, T., Black silicon for solar cell applications, Proceedings of SPIE Vol. 8438, 843817 (2012).

[224] Nekarda, J., Graf, M., Rodofili, A., Wolf, A. and Preu, R., Laser based foil rear side metallization for crystalline silicon solar cells, Proceedings of SPIE Vol. 8473, Laser Material Processing for Solar Energy, (2012).

[225] Grohe, A., Nekarda, J. and Schultz, O., Verfahren zur Metallisierungvon Solarzellen und dessen Verwendung, Patent, DE 102006044936 B4, (2008).

[226] Nekarda, J., Preu, R., Hauser, H. and Bläsi, B., Laserbasiertes Verfahren und Bearbeitungstisch zur Metallisierung eines Halbleiterbauelements, Patent, patent filed, (2012). 


\section{List of Symbols and Acronyms}

\begin{tabular}{|c|c|c|}
\hline Symbol & Description & Unit \\
\hline a & lattice constanct & $\mathrm{m}$ \\
\hline$d$ & thickness of coatings & $\mathrm{m}$ \\
\hline$D$ & diffusion constant & \\
\hline$D(E)$ & density of states as a function of energy level & $\mathrm{eV}^{-1} \mathrm{~m}^{-3}$ \\
\hline$D_{i t}(E)$ & density of interface states as a function of energy level & $\mathrm{eV}^{-1} \mathrm{~m}^{-2}$ \\
\hline$E_{C}$ & energy level of conduction band edge & $\mathrm{eV}$ \\
\hline$E_{F}$ & Fermi energy & $\mathrm{eV}$ \\
\hline$E_{F, n}, E_{F, p}$ & quasi-Fermi energy (for electrons and holes) & $\mathrm{eV}$ \\
\hline$E_{G a p}$ & band gap & $\mathrm{eV}$ \\
\hline$E_{t}$ & energy level of defects & $\mathrm{eV}$ \\
\hline$E_{V}$ & energy level of valence band edge & $\mathrm{eV}$ \\
\hline$f$ & fractional area of metallisation for point contacts & $\%$ \\
\hline$F(E)$ & Fermi-Dirac distribution & \\
\hline$F F$ & fill factor & $\%$ \\
\hline$G$ & optical generation rate & $\mathrm{cm}^{-3} \mathrm{~s}^{-1}$ \\
\hline$J$ & electric current & A \\
\hline$j_{0}$ & dark saturation current density & $\mathrm{A} / \mathrm{m}^{2}$ \\
\hline$J_{0}$ & dark saturation current & A \\
\hline$j_{0 e}, j_{0 b}$ & dark saturation current density in emitter or bulk & $\mathrm{A} / \mathrm{m}^{2}$ \\
\hline$J_{M P P}$ & electric current at maximum power point & A \\
\hline$j_{p h}$ & photo-generated current density & $\mathrm{A} / \mathrm{m}^{2}$ \\
\hline$J_{p h}$ & photo-generated current & A \\
\hline$j_{s c}$ & short-circuit current density & $\mathrm{A} / \mathrm{m}^{2}$ \\
\hline$J_{s c}$ & short-circuit current & A \\
\hline
\end{tabular}




\begin{tabular}{|c|c|c|}
\hline$I$ & intensity & $\mathrm{W} / \mathrm{m}^{2}$ \\
\hline$k$ & wave-vector & $m^{-1}$ \\
\hline$L_{n}, L_{p}$ & diffusion length (for electrons and holes) & $\mathrm{m}$ \\
\hline$\lambda$ & wavelength & $\mathrm{m}$ \\
\hline$\lambda_{0}$ & vacuum wavelength & $\mathrm{m}$ \\
\hline$\Lambda$ & structure period & $\mathrm{m}$ \\
\hline$n$ & refractive index & \\
\hline$n, p$ & density of free electrons or holes & $\mathrm{m}^{-3}$ \\
\hline$\Delta n$ & excess carrier density & $m^{-3}$ \\
\hline$n_{i}$ & intrinsic carrier density & $m^{-3}$ \\
\hline$n_{1}, p_{1}$ & Shockley-Read-Hall electron and hole density & $m^{-2}$ \\
\hline$n_{s}, p_{s}$ & concentrations of electrons or holes at the surface & $m^{-2}$ \\
\hline$N_{C}, N_{V}$ & effective density of states (in conduction / valence band) & $m^{-3}$ \\
\hline$p$ & pressure & $\mathrm{Pa}$ \\
\hline$P_{\text {Irradiance }}$ & power of irradiance & $\mathrm{W} / \mathrm{m}^{2}$ \\
\hline$P_{M P P}$ & electric power density at maximum power point & $\mathrm{W} / \mathrm{m}^{2}$ \\
\hline$\Phi(\lambda)$ & photon flux as a function of wavelength & $\mathrm{m}^{-3} \mathrm{~s}^{-1}$ \\
\hline$\Psi_{i}$ & wave-function & \\
\hline$R$ & recombination rate & $m^{-3} s^{-1}$ \\
\hline$R_{\text {Surf }}$ & surface recombination rate & $m^{-2} s^{-1}$ \\
\hline$R(\lambda)$ & spectral reflectivity & $\% / m$ \\
\hline$R_{w}$ & weighted reflectance & $\%$ \\
\hline$\sigma_{n}, \sigma_{p}$ & capture cross section of defects (for electrons / holes) & $m^{2}$ \\
\hline$S$ & surface recombination velocity & $\mathrm{m} / \mathrm{s}$ \\
\hline$S_{\text {front }}, S_{\text {back }}$ & surface recombination velocity (front or back surface) & $\mathrm{m} / \mathrm{s}$ \\
\hline$S_{m e t}$ & surface recombination velocity in metallised areas & $\mathrm{m} / \mathrm{s}$ \\
\hline$S_{\text {pass }}$ & surface recombination velocity in passivated areas & $\mathrm{m} / \mathrm{s}$ \\
\hline$T$ & temperature & ${ }^{\circ} \mathrm{C}$ \\
\hline
\end{tabular}




\begin{tabular}{lll}
\hline \hline$\tau$ & minority carrier lifetime & $\mathrm{s}$ \\
$\tau_{\text {Auger }}$ & minority carrier lifetime for Auger recombination & $\mathrm{s}$ \\
$\tau_{\text {eff }}$ & effective minority carrier lifetime & $\mathrm{s}$ \\
$\tau_{\text {radiative }}$ & minority carrier lifetime for radiative recombination & $\mathrm{s}$ \\
$\tau_{S R H}$ & minority carrier lifetime for Shockley-Read-Hall & $\mathrm{s}$ \\
& recombination & \\
$\tau_{\text {surface }}$ & minority carrier lifetime for surface recombination \\
$\theta_{c r i t}$ & critical angle for total internal reflection & $\mathrm{s}$ \\
$v_{\text {th }}$ & thermal velocity & $\circ$ \\
$V_{M P P}$ & voltage at maximum power point & $\mathrm{m} / \mathrm{s}$ \\
$V_{\text {oc }}$ & open-circuit voltage & $\mathrm{V}$ \\
$W$ & wafer thickness & $\mathrm{V}$ \\
\hline \hline
\end{tabular}

\begin{tabular}{ll}
\hline \hline Acronym & Description \\
\hline AFM & atomic force microscopy \\
ALD & atomic layer deposition \\
AM1.5g & air mass 1.5 global spectrum \\
ARC & antireflection coating \\
BARC & bottom-antireflection coating \\
BSF & back surface field \\
COC & cyclic olefin copolymer \\
Cz & Czochralsky method (fabrication of monocrystalline silicon) \\
DARC & double layer antireflection coating \\
DNQ & diazonaphthoquinone \\
DoE & design of experiments \\
DOE & diffractive optical element \\
E-beam & electron beam lithography \\
ECR & electron cyclotron resonance \\
\hline \hline
\end{tabular}




\begin{tabular}{|c|c|}
\hline EDX & energy-dispersive $\mathrm{X}$-ray spectroscopy \\
\hline EQE & external quantum efficiency \\
\hline ETFE & ethylene tetrafluoroethylene \\
\hline FCA & free-carrier absorption \\
\hline FEM & finite element method \\
\hline FZ & float zone (fabrication of monocrystalline silicon) \\
\hline GWP & global warming potential \\
\hline HEL & hot embossing lithography \\
\hline HF & hydrofluoric acid \\
\hline $\mathrm{HNO}_{3}$ & nitric acid \\
\hline ICP-OES & inductively coupled plasma optical emission spectrometry \\
\hline IQE & internal quantum efficiency \\
\hline $\mathrm{KOH}$ & potassium hydroxide \\
\hline LED & light emitting diode \\
\hline LFC & laser-fired contacts \\
\hline LIGA & $\begin{array}{l}\text { german acronym for Lithographie, Galvanoformung, } \\
\text { Abformung }\end{array}$ \\
\hline MEMS & micro-electro-mechanical systems \\
\hline MPP & maximum power point \\
\hline MW & microwave \\
\hline NIL & nanoimprint lithography \\
\hline NMP & N-Methyl-2-pyrrolidone \\
\hline OMA & optical multichannel analyser \\
\hline PDMS & polydimethylsiloxane \\
\hline PECVD & plasma-enhanced chemical vapour deposition \\
\hline PERC & passivated emitter and rear cell (solar cell structure) \\
\hline PERL & passivated emitter rear locally diffused (solar cell structure) \\
\hline PERT & passivated emitter rear totally diffused (solar cell structure) \\
\hline PMMA & poly(methyl methacrylate) \\
\hline
\end{tabular}




\begin{tabular}{ll}
\hline \hline PSG & phosphorus silicate glass \\
PV & photovoltaics \\
QSSPC & quasi-steady state photoconductance \\
RCWA & rigorous-coupled wave analysis \\
RF & radio frequency \\
RIE & reactive ion etching \\
SARC & single layer antireflection coating \\
SEM & scanning electron microscopy \\
SLAN & slot-antenna \\
SRH & Shockley-Read-Hall (lifetime or recombination) \\
UV & ultraviolet \\
\hline \hline
\end{tabular}




\section{List of Publications and Patents}

\section{Publications}

- A. Mellor, H. Hauser, Ch. Wellens, J. Benick, J. Eisenlohr, M. Peters, A. Guttowski, I. Tobías, A. Martí, A. Luque, B. Bläsi, Nanoimprinted diffraction gratings for crystalline silicon solar cells: implementation, characterisation and simulation, Optics Express, 21 (S2), A295-A304, (2013).

- H. Hauser, B. Michl, Ch. Walk, J. Eisenlohr, J. Benick, A. Mellor, C. Müller, M. Hermle, B. Bläsi, Full-Wafer Roller-NIL processes for solar cell texturisation, Proceedings of the $27^{\text {th }}$ PVSEC, Frankfurt (2012).

- A. Mellor, H. Hauser, A. Guttowski, Ch. Wellens, B. Bläsi, I. Tobías, A. Martí, A. Luque, Nano-imprinted rear-side diffraction gratings for absorption enhancement in solar cells, Proceedings of the SPIE Solar Energy + Technology. 0001;():847100-847100-7 (2012). doi:10.1117/12.929034.

- B. Bläsi, H. Hauser, A. J. Wolf, Photon management structures for solar cells, SPIE Newsroom, June (2012) DOI: 10.1117/2.1201205.004241

- H. Hauser, A. Mellor, A. Guttowski, Ch. Wellens, J. Benick, C. Müller, M. Hermle, B. Bläsi, Diffractive Backside Structures via Nanoimprint Lithography, Energy Procedia, Vol. 27 , p. 337-342 (2012).

- B. Bläsi, H. Hauser, C. Walk, B. Michl, A. Guttowski, A. Mellor, J. Benick, M. Peters, S. Jüchter, Photon Management Structures for Solar Cells, Proc. SPIE Photonics Europe (2012).

- S. Jüchter, H. Hauser, Ch. Wellens, A. Guttowski, M. Peters, J.C. Goldschmidt, U.T. Schwarz, B. Bläsi, Preparation of periodically arranged metallic nanostructures using nanoimprint lithography, Proc. SPIE Photonics Europe (2012).

- S. Jüchter, H. Hauser, Ch. Wellens, M. Peters, J.C. Goldschmidt, U.T. Schwarz, B. Bläsi, Metallic nanostructures prepared by nanoimprint lithography for application in solar cells, Proc. EOS Diffractive Optics Conference (2012).

- H. Hauser, B. Michl, S. Schwarzkopf, V. Kübler, C. Müller, M. Hermle, and B. Bläsi, Honeycomb Texturing of Silicon Via Nanoimprint Lithography for Solar Cell Applications, IEEE JOURNAL OF PHOTOVOLTAICS, VOL.2, NO.2, April (2012).

- A. J. Wolf, H. Hauser, V. Kübler, Ch. Walk, O. Höhn, B. Bläsi, Origination of nano- and microstructures on large areas by interference lithography, Microelectronic Engineering, Vol. 98, p. 293-296 (2012). 
- B. Bläsi, H. Hauser, Ch. Walk, B. Michl, V. Kübler, A.J. Wolf, Photon Management Structures Based on Interference Lithography, Future Photovoltaics (2011).

- B. Bläsi, H. Hauser, J. Benick, A. Mellor, A. Guttowski, S. Jüchter, Ch. Wellens, M. Peters, V. Kübler, A.J. Wolf, Photon Management Structures for Solar Cells - From Modeling to Fabrication, Proc. EOS Micro- and NanoOptoelectronic systems, Bremen (2011).

- B. Bläsi, H. Hauser, Ch. Walk, B. Michl, A. Mellor, A. Guttowski, S. Jüchter, Ch. Wellens, M. Peters, V. Kübler, A. J. Wolf, Photon Management Structures Based on Interference Lithography and Nanoimprint Processes, Proceedings of the 26th PVSEC, Hamburg (2011).

- M. Peters, M. Rüdiger, H. Hauser, M. Hermle and B. Bläsi, Diffractive gratings for crystalline silicon solar cells-optimum parameters and loss mechanisms, Prog. Photovolt: Res. Appl. (2011), DOI: 10.1002/pip.1151.

- H. Hauser, B. Bläsi, Photonenmanagement in der Photovoltaik durch strukturierte Oberflächen, inno-magazin 16. Jahrgang, Nr. 48, März (2011).

- H. Hauser, B. Bläsi, Oberflächentexturen für hocheffiziente Solarzellen durch Nanoimprint-Lithographie, in W.D. Prenzel (Editor), Jahrbuch Optik und Feinmechanik, ISBN 978-3-7949-8017-2 (2011).

- H. Hauser, B. Michl, V. Kübler, S. Schwarzkopf, C. Müller, M. Hermle, B. Bläsi, Nanoimprint Lithography for Honeycomb Texturing of Multicrystalline Silicon, Energy Procedia 8 648-653 (2011).

- B. Bläsi, H. Hauser, O. Höhn, V. Kübler, M. Peters, A. J. Wolf, Photon Management Structures Originated by Interference Lithography, Energy Procedia 8, 712-718 (2011).

- H. Hauser, B. Michl, S. Schwarzkopf, V. Kübler, C. Müller, M. Hermle and B. Bläsi, Nanoimprint lithography for solar cell texturisation, The 9th International Conference on Nanoimprint and Nanoprint Technology, Copenhagen, October 13-15 (2010).

- H. Hauser, B. Michl, S. Schwarzkopf, C. Müller, M. Hermle and B. Bläsi, Development of nanoimprint processes for solar cell texturisation, Proceedings of the 25th PVSEC, Valencia (2010).

- M. Peters, M. Rüdiger, D. Pelzer, H. Hauser, M. Hermle, B. Bläsi, Electrooptical modelling of solar cells with photonic structures, Proceedings of the 25th PVSEC, Valencia (2010).

- P. Berger, Hubert Hauser, D. Suwito, S. Janz, M. Peters, B. Bläsi, M. Hermle, Realisation and evaluation of diffractive systems on the back side of silicon solar cells, Proc. SPIE Photonics Europe (2010). 
- H. Hauser, P. Berger, B. Michl, C. Müller, S. Schwarzkopf, M. Hermle, B. Bläsi, Nanoimprint Lithography for Solar Cell Texturisation, Proc. SPIE Photonics Europe (2010).

- M. Peters, J. C. Goldschmidt, M. Rüdiger, P. Voisin, H. Hauser, M. Hermle, B. Bläsi, Diffractive gratings in solar cells and how to model them, Quantsol Winter Workshop (2010).

- M. Peters, A. Bielawny, B. Bläsi, R. Carius, S.W. Glunz, J.C. Goldschmidt, H. Hauser, M. Hermle, T. Kirchartz, P. Löper, J. Üpping, R. Wehrspohn, G. Willeke, Photonic Concepts for Solar Cells, in V. Badescu (Editor), Physics of Nanostructured Solar Cells, Nova Science Publishers (2009).

- P. Voisin, M. Peters, H. Hauser, C. Helgert, E.-B. Kley, T. Pertsch, B. Bläsi, M. Hermle, S.W. Glunz, Nanostructured back side silicon solar cells, Proceedings of the 24th PVSEC, Hamburg (2009).

- H. Hauser, P. Voisin, A. Guttowski, J. Mick, M. Pfeifer, C. Müller, M. Hermle, S. Glunz and B. Bläsi, Honeycomb textured multicrystalline silicon via nanoimprint lithography, Proceedings of the 24th PVSEC, Hamburg (2009).

\section{Patents}

- A. Knorz, R. Preu, M. Peters, H. Hauser, B. Bläsi, Verfahren zum Materialabtrag an Festkörpern mit Herabsetzen der zeitlichen und/oder räumlichen Kohärenz der Laserstrahlung (DE 102010026331 A1, disclosed 11.01.2012).

- M. Hermle, B. Bläsi, M. Peters, J.C. Goldschmidt, H. Hauser, P. Voisin, Siliziumsolarzelle mit nanostrukturierter a-Si Rückseite (DE102009042018A1, disclosed 24.03.2011).

- B. Bläsi, M. Peters, J.C. Goldschmidt, M. Hermle, H. Hauser, P. Voisin, Solarzelle und Verfahren zu deren Herstellung (DE102009029944A1, disclosed 23.12.2010).

- J. Nekarda, R. Preu, H. Hauser, B. Bläsi, Laserbasiertes Verfahren und Bearbeitungstisch zur Metallisierung eines Halbleiterbauelements, patent filed (2012). 


\section{Danksagung}

An dieser Stelle einen herzlichen Dank an alle, die während der letzten Jahre auf vielfältige Weise zum Gelingen dieser Arbeit beigetragen haben. Mein besonderer Dank gilt:

Prof. Dr. H. Reinecke und Prof. Dr. G. Willeke für die Bereitschaft diese Arbeit als Gutachter zu betreuen,

Prof. Dr. Claas Müller und Dr. Benedikt Bläsi für ihre intensive und konstruktive Betreuung der Arbeit und für die Möglichkeit im Rahmen dieser Arbeit sehr viel von ihnen zu lernen,

Dr. Benedikt Bläsi für die Möglichkeit diese Doktorarbeit in einer tollen Atmosphäre in der Gruppe "Mikrostrukturierte Oberflächen" am Fraunhofer ISE anzufertigen, seine starke Unterstützung sowie die Freiheit, eigene Ideen einzubringen und zu verfolgen,

Dr. Stefan Glunz und Dr. Martin Hermle danke ich für die Unterstützung und das Interesse an den Entwicklungen dieser Arbeit seitens des Bereichs "Solarzellen Entwicklung und Charakterisierung". Hierbei auch ein sehr großes Dankeschön an Bernhard Michl, Dr. Pauline Berger, Dr. Jan Benick und Johannes Eisenlohr für einige Nachhilfestunden zur Prozessierung von Solarzellen und ihren Einsatz die gemeinsamen Entwicklungen voranzutreiben,

Aron Guttowski, Sebastian Schwarzkopf und Christian Walk für ihre motivierte und motivierende Zeit, in der sie ihre Abschlussarbeiten bestritten und die in dieser Arbeit beschriebenen Entwicklungen vorantrieben,

allen Kollegen aus der Gruppe Mikrostrukturierte Oberflächen. Hierbei seien stellvertretend genannt: Andreas Wolf, Volker Kübler, Dr. Christine Wellens, Oliver Höhn, Michael Nitsche, Sabrina Jüchter, Dr. Marius Peters und natürlich alle HiWi's für unzählige REM Aufträge und Stempelherstellungen,

allen weiteren Wiesentälern für eine äußerst angenehme und kreative Arbeitsatmosphäre, die Unterstützung sowie das gegenseitige Interesse an Fragestellungen, Problemchen und Durchbrüchen (auch aufgrund der maroden Infrastruktur...). Stellvertretend für einige seien hier genannt: Franz Brucker, Josef Steinhart, Dr. Thomas Kroyer, Walter Schnetzler, Dr. Andreas Georg, Wolfgang Graf, Dr. Armin Zastrow,

Alex Mellor von der Universidad Politécnica de Madrid, der während als GastDoktorand in der Gruppe Mikrostrukturierte Oberflächen viel Energie in die Herstellung der diffraktiven Rückseitengitter steckte. Des Weiteren für seine 
wellenoptischen Modellierungen der hier realisierten Gitter, seine freundliche Art, spannende Diskussionen und natürlich die harte (und sicherlich hier und da schmerzhafte) Arbeit des Korrekturlesens als Muttersprachler. Seine Korrekturen machten aus einem Deutsch-Englisch dieses Dokument,

Andreas Wolf und Paul Walden für die ein oder andere AFM Messung,

Volker Kübler für Belichtungen im Interferenzlithographie-Labor sowie für fast immer konstruktive Anregungen,

Christian Schetter und Harald Steidl für die oft sehr kurzfristigen Hilfestellungen bei technischen Problemchen an der Plasmaätzanlage wie auch Tipps und Tricks rund ums Prozessieren daran,

Sonja Seitz für photolithographische Belichtungen sowie einige Diskussion über das Prozessieren von Photolacken,

Dr. Benedikt Bläsi, Prof. Dr. Claas Müller, Alex Mellor, Bernhard Michl, Lutz Labusch, Volker Kübler, Andreas Wolf, Dr. Jan Benick, Dr. Martin Hermle, Dr. Jan Christoph Goldschmidt, Dr. Thomas Kroyer und Dr. Stefan Glunz für immer wieder wichtige Diskussionen sowie die hilfreichen und wertvollen Korrekturen dieser Arbeit,

meinen Eltern, die mir das Studium der Mikrosystemtechnik und somit diese Promotion überhaupt erst ermöglicht haben,

meiner Frau Nadine und unserem Sohnemann Janosch für die wundervolle Unterstützung sowie das schier endlose Verständnis wenn es doch mal wieder später wurde und der Kopf nicht immer frei war. 